\title{
Three USGS Mafic Rock Reference Samples, W-2, DNC-1, and BIR-1
}

\section{U.S. GEOLOGICAL SURVEY BULLETIN 1623}

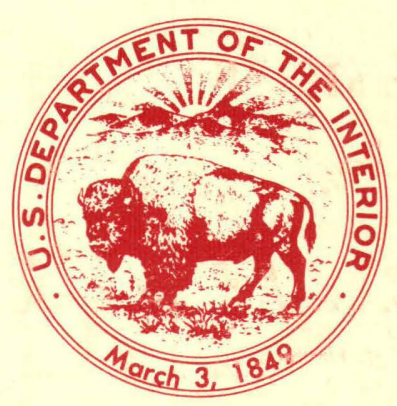





\section{Three USGS Mafic Rock Reference Samples, W-2, DNC-1, and BIR-1}

By F. J. Flanagan

Three mafic rock reference samples to furnish calibration points for trace-element data between mafic and ultramafic reference samples were prepared

U.S. GEOLOGICAL SURVEY BULLETIN 1623 


\title{
DEPARTMENT OF THE INTERIOR WILLIAM P. CLARK, Secretary
}

\author{
U.S. GEOLOGICAL SURVEY \\ Dallas L. Peck, Director
}

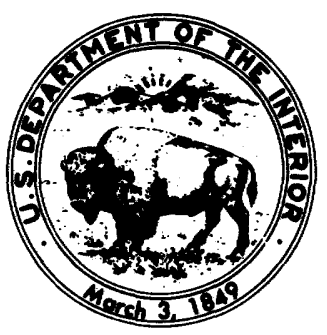

For sale by Distribution Branch

Text Products Section

U.S. Geological Survey

604 South Pickett Street

Alexandria, Virginia 22304

Library of Congress Cataloging in Publication Data

Flanagan, F. J. (Francis James), 1915-

Three USGS mafic rock reference samples, W-2, DNC-1, and BIR-1.

(U.S. Geological Survey bulletin ; 1623)

Bibliography: $p$.

1. Alkalic igneous rocks. I. Title. II. Title: Three U.S.G.S.

mafic rock reference samples, $\mathrm{W}-2, \mathrm{DNC}-1$, and

BIR-1. III. Series. QE75.B9 no. 1623 [QE462.A4] $557.3 \mathrm{~s}$

[552'.1] 84-10160 


\title{
CONTENTS
}

\author{
Abstract 1 \\ Introduction 1 \\ Diabase W-2 2 \\ Dolerite DNC-1 2 \\ Icelandic basalt BIR-1 2 \\ Sample processing 2 \\ Tables of data 3 \\ Analysis of variance 3 \\ Calculations of best values 4 \\ Best values 5 \\ Compositions of W-1 and W-2 7 \\ The rare-earth elements 9 \\ Future analytical work 10 \\ References 11
}

\section{FIGURE}

1. Best values for rare-earth elements normalized to chondritic abundances 10

\section{TABLES}

1. Analytical data for USGS-W-2 13

2. Analytical data for USGS-DNC-1 26

3. Analytical data for USGS-BIR-1 38

4. Determinations of $\mathrm{Ni}, \mathrm{Rb}$, and $\mathrm{Sr}$ by $\mathrm{X}$-ray fluorescence spectroscopy using two X-ray systems, by M. Quintin $\mathbf{5 3}$

5. Determinations of trace elements in USGS-BIR-1 by spark source mass spectrometry, by K. P. Jochum and M. Seufert, Max Planck Institut für Chemie 53

6. Determinations of constituents by instrumental neutron activation analysis, by A. V. Murali 54

7. Calculation of a homogeneous variance for $\mathrm{Zr}$ data in W-2 5

8. Calculations for the best value, $\overline{\overline{\mathrm{x}}}$, the grand mean, for $\mathrm{Zr}$ data in sample $\mathrm{W}-2$ by the Studentized range 5

9. Best estimates of the compositions of samples W-1, W-2, DNC-1, and BIR-1 6

10. Data for several elements by isotope dilution mass spectrometry 8

11. Contributions of individual constituents to $\chi^{2} 8$

12. Laboratory averages for several constituents of $\mathrm{W}-2 \quad 9$

13. Some trace element contents of USGS mafic and ultramafic samples 11 


\section{Three USGS Mafic Rock Reference Samples, W-2, DNC-1, and BIR-1}

\author{
By F. J. Flanagan
}

\begin{abstract}
Analytical data and best values are presented for three new U.S. Geological Survey mafic rock reference samples: W-2, another portion of the diabase at Centreville, Va., which replaces $\mathrm{W}-1$; DNC-1, a dolerite from North Carolina; and BIR-1, an Icelandic basalt. The supplies of each powdered sample are expected to last 30 years or more. Best values for an oxide or element in a sample were calculated by the sequential procedure of (1) calculating a homogeneous variance for sets of six determinations for a constituent, (2) calculating from this variance the standard deviation of the means of six determinations, and (3) using this standard deviation to Studentize the range of means to decide which means could have been derived from the same population.

The chondrite-normalized abundances of rare-earth elements, calculated from best values for the three samples, show that the contents of light rare-earth elements are low in DNC-1 and are very low in BIR-1. Future data by isotope dilution mass spectrometry will result in smoother rare-earth element plots for the three samples. In addition to data for the light rare-earth elements, there are best values or magnitudes for other trace elements whose contents decrease with decreasing amounts of $\mathrm{K}_{2} \mathrm{O}$ in the samples. Data for other elements useful in geochemical studies are desirable, especially for samples DNC-1 and BIR-1. Determinations of several elements, including gold and the platinum group metals, were not reported.
\end{abstract}

\section{INTRODUCTION}

The three U.S. Geological Survey mafic rocks generally used as standards for low-level concentrations of residual or lithophilic trace elements are $\mathrm{W}-1$, from the diabase at Centreville, Va., BCR-1 from the Columbia River Basalt Group, and BHVO-1, a Hawaiian basalt. The trace element contents of these three standards differ greatly from those of the ultramafic samples, peridotite PCC-1 and dunite DTS-1 (the Twin Sisters dunite; Ragan, 1963). Unfortunately, the supply of W-1 was exhausted in 1972, and supplies of BCR-1 and DTS-1 were so low that they were removed from the list of available samples in late 1975. Because we needed not only to replace the mafic and ultramafic samples but also to provide trace element data in the compositional gap between mafic and ultramafic reference samples, we considered three gabbro samples that have been processed in the last decade.

The Mont-Royal Gabbro, MRG-1, was described by Perrault and others (1974); Abbey (1980) listed usable values for 38 trace elements, adding a question mark after data for 23 elements to indicate some uncertainty in the values. Perrault and others (1974) described the sample as an augite-olivine gabbro, but the sample appears not to be as petrologically important as other gabbros that have a greater geographic distribution.

Data for another gabbro, GOG-1 (Gruppo Ofioliti, Gabbro, 1), were published by Boy and Mazzucotelli (1976) and by Mazzucotelli and others (1976). Benedetti and others (1977) have summarized data for 10 major and minor oxides and for 17 trace elements as averages and standard deviations. Neither a description of the sample nor the location from which the sample was collected has been published. Sample GOG-1 is from a northern Appenine ophiolite sequence, the Bracco Massif (A. Mazzucotelli, written commun., 1980). Only limited analytical data, including those reported by Harris and others (1981), are available for this sample.

The third gabbro, USGS-GSM-1, is a sample of the San Marcos Gabbro (Miller, 1937) described by Larsen (1948). Most published data for GSM-1 appear in Harris and others (1981).

Thus, problems of some type are associated with all three gabbros. We therefore decided to recollect a large amount of the diabase at Centreville, which had been used by geochemists for three decades, and to collect large quantities of two other samples, which contained elemental concentrations similar to those in island-arc tholeiites, ocean-ridge basalts, and primitive continental tholeiites and hence could furnish two calibration points between the diabase at Centreville and the ultramafic rocks. 


\section{DIABASE $\mathbf{W}-\mathbf{2}$}

Diabase W-1 was a valuable reference sample until the supply was exhausted in 1972; it was decided to replace the sample with material from the same source. Eight hundred pounds $(\sim 360 \mathrm{~kg})$ of the rock was collected in late 1976 from the Bull Run (now Luck) Quarry on U.S. Route 29-211, about $3 \mathrm{mi}(5 \mathrm{~km})$ west-southwest of Centreville, Va. The sample was collected at the foot of the northeast wall of the quarry, about $650 \mathrm{ft}(200 \mathrm{~m})$ from Route 29-211. Care was taken to collect fresh material recently blasted from the wall of the quarry, avoiding any pieces that showed alteration products, such as chlorite, or small inclusions not part of the diabase.

Hand specimens of the rock for W-1 and for W-2, the present sample, are indistinguishable; the petrography of the rock was discussed by Chayes (1951). The entire lot of sample was processed and was numbered $\mathrm{W}-2$. About $2500 \quad 1-\mathrm{oz}(30-\mathrm{mL})$ bottles were filled for distribution; the rest of the powdered rock was stored in plastic bags placed inside cardboard boxes.

\section{DOLERITE DNC-1}

Another sample believed to be important because of the low levels of some lithophilic trace elements was one of the Triassic-Jurassic olivine-normative dolerites from North Carolina discussed by Ragland and others (1968). P. C. Ragland and J. R. Butler of the Department of Geology, University of North Carolina, Chapel Hill, collected and shipped about $500 \mathrm{lb}(\sim 230 \mathrm{~kg})$ of a homogeneous rock known locally as the Braggtown dolerite. The entire lot of sample was powdered, and the excess after filling 2500 bottles was stored in the same manner as the excess of $\mathrm{W}-2$.

There may be a problem in the future for someone who wishes to duplicate this sample. J. R. Butler (written commun., 1980) noted that the Braggtown Quarry, the site of the sample, located immediately above the word Braggtown $\left(78^{\circ} 53^{\prime} 45^{\prime \prime} \mathrm{W}, 36^{\circ} 01^{\prime} 55^{\prime \prime} \mathrm{N}\right)$ in the southeast quarter of the Northwest Durham 7.5-min quadrangle (topographic), is on land owned by the State Museum of Life and Science. Since the material for DNC-1 was collected, the Museum has erected a fence around the quarry so that the enclosed area can be used as a natural habitat for bears.

\section{ICELANDIC BASALT BIR-1}

Shortly after the publication of "Reference samples for the earth sciences" (Flanagan, 1974), in which a primitive basalt standard was suggested, Karl Gronwold of the Nordic Volcanological Institute at
Reykjavik volunteered to supply a basalt from Iceland; a sample of about $500 \mathrm{lb}(\sim 230 \mathrm{~kg})$ of a basalt was received and was processed as BIR-1. Gronwold furnished the following description of the sample:

"The chemical compositions of Icelandic basalts cover the range from typical abyssal tholeites to alkali basalts. The sample was collected from that part of the chemical spectrum which is identical to abyssal tholeiites.

"The source of the sample is one of the interglacial lava flows often referred to as the Reykjavik dolerites. The sample locality is a low hill about $10 \mathrm{~m}$ above the surroundings at a height of $100 \mathrm{~m}$ a.s.l. [above sea level]. The site is about $12 \mathrm{~km}$ east of Reykjavik and $800 \mathrm{~m}$ from the main road.

"The Reykjavik dolerites are a group of lava flows most likely from shield volcanos dating from the youngest interglacial periods. The source crater for the sampled flow is buried by younger lavas. The rock is a coarsegrained olivine tholeiite, and the available data show that the individual flows are chemically heterogeneous.

"The sample was collected from three adjoining blocks found in situ within an area of $4 \mathrm{~m}^{2}$. The surface of the flow has been removed by glacial erosion. The large pieces of the sample were taken to the laboratory and were broken with a sledge hammer. The resulting pieces were trimmed and weathered surfaces were removed before shipment to the USGS."

After the rock was received by the USGS, I found that many pieces had to be broken by a small sledge hammer before they could fit between the plates of a jaw crusher. As with samples W-2 and DNC-1, all material shipped, except for a few hand specimens, was powdered, and the excess after filling 2500 bottles was stored.

\section{SAMPLE PROCESSING}

The supplies of samples DNC-1 and BIR-1 are expected to last about 30 years and the supply of W-2 somewhat longer because of the larger amount of rock. Because of the amount of analytical work that may be done over these anticipated lifetimes, extra precautions were introduced into the general procedure (Flanagan, 1967) for processing rock standards.

The primary contaminant of processed rock samples is free iron $\left(\mathrm{Fe}^{0}\right)$ from the jaw crusher. This can be introduced when the distance between the bottom edges of the crusher plates is set at the minimum $(\sim 1 / 8 \mathrm{in}$., or $3 \mathrm{~mm}$ ). If too much rock is added to the crusher, some partly crushed material will not pass between the bottom edges of the plates, and the addition of more rock will cause additional partly crushed material to accumulate above these edges. These partly crushed pieces will be scraped continuously by the plates until the material is released. 
To reduce possible contamination by free iron, the entire batch of a sample was first crushed with the plates separated by the maximum distance $(\sim 3 / 8 \mathrm{in}$., or $10 \mathrm{~mm})$. The bottom edges were then adjusted to about $1 / 8$ in., and material from the preliminary crushing was passed through the narrower gap.

The roller crusher was also used in a two-stage operation. Material from the jaw crushing was initially passed between rollers whose contact surfaces were about $1 / 8$ in. apart. The product was then passed between the rollers set at zero separation.

The material for each batch of $135 \mathrm{lb}(\sim 60 \mathrm{~kg})$ of crushed sample was processed in the ball mill until about 95 percent of a half pint $(0.25 \mathrm{~L})$ of sample withdrawn for testing passed a 200-mesh $(0.074-\mathrm{mm})$ sieve. The material withdrawn for size testing was discarded. Several roughly spherical pieces of rock having an effective diameter of $0.5 \mathrm{~cm}$ or less were observed occasionally. These pieces could not be distinguished from the original rock. All material of the three powdered samples was therefore passed over a piece of 16-mesh $(0.99-\mathrm{mm})$ by 18 -mesh $(0.90-\mathrm{mm})$ aluminum screen, and the oversize material, which amounted to less than $50 \mathrm{~g}$ per $250-\mathrm{kg}$ sample, was removed.

To estimate the final particle-size distribution, the contents of seven bottles from the randomly ordered stock of each of the three powdered samples were combined for sieve tests. The powdered material and the sieves were dried in an oven at about $105^{\circ} \mathrm{C}$ for more than an hour before screening; the size distributions obtained were as follows:

Particle size distribution

[In weight percent; $t r$, trace]

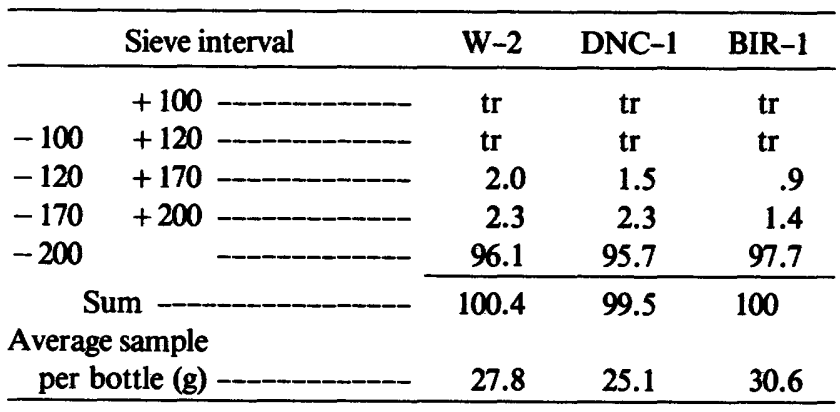

There has been renewed emphasis in the last 15 years by Langmyhr (1969) and Steele (1978) to report analytical data on samples dried to remove hygroscopic water or to report data with $\mathrm{H}_{2} \mathrm{O}^{-}$determined on a separate portion. The air in the grinding rooms in which the samples were prepared is often humid, and I decided to report the $\mathrm{H}_{2} \mathrm{O}^{-}$contents of the samples shortly after they were prepared. Accordingly, 1-g portions of the three samples were dried overnight at $110^{\circ} \mathrm{C}$ in weighing bottles; they yielded the following preliminary estimates.

\begin{tabular}{lc}
\hline $\mathrm{H}_{2} \mathrm{O}^{-}$ & (weight percent) \\
\hline $\mathrm{W}-2-1$ & 0.29 \\
DNC-1 & .37 \\
$\mathrm{BIR}-1-06$ \\
\hline
\end{tabular}

\section{TABLES OF DATA}

Data by the 81 analysts and 33 organizations who contributed so generously are found in tables 1-3 (p. 13-48). The data in these tables are presented in the general order of conventional and rapid [principally atomic-absorption spectroscopic (AAS)] methods of rock analysis, X-ray fluorescence spectroscopy (XRF), and instrumental neutron-activation analysis (INAA). Three analysts used some type of plasma excitation technique. Data by dc-arc optical emission spectroscopy (DC-OES) were included with chemical analyses by one organization but were reported separately by other analysts. Data by spark-source mass spectrometry (SSMS) and determinations of cadmium by isotope dilution mass spectrometry (IDMS) are reported for the samples. Single, or occasionally duplicate, determinations by IDMS are given for barium, lead, and some rare-earth elements. Some major and minor oxides were reported as elements. These data were converted to oxides for calculating best values.

Uranium and thorium determined by delayed neutron-activation analysis (DNAA) were reported by two groups of analysts, but the uranium and thorium contents of DNC-1 and BIR-1 are below the detection limits of the methods. Tables 4-6 (p. 53-54) show data obtained by XRF, by SSMS using two methods of calibration, and by INAA; these follow the large tables as the data did not fit the general scheme for the three large tables.

\section{ANALYSIS OF VARIANCE}

The analysis of variance of the data that were amenable to the technique was made by several analysts. I repeated these calculations and also calculated the analyses of variance for the remaining data. Some changes were necessary for several sets of data. For example, two of the three INAA determinations of $\mathrm{Ba}$ for bottle 3 of DNC-1 in table 2 are listed as having lower limits of 200 and $250 \mathrm{ppm}$. As data on this ordinal scale of measurement (S. S. Stevens, 1946) are not amenable to the analysis of variance, the last datum for bottle 3 was discarded and the analysis of variance was made on the remaining six data. The necessary changes in the degrees of freedom are indicated in notes in a section on abbreviations and analytical methods for tables 1-3. 
One analyst reported three determinations of $\mathrm{Ce}$ on portions of three bottles of W-2 and each of the first determinations on the bottles is asterisked. The first determinations for bottles 1 and 3 obviously did not belong to the same population of data as the remaining data and were discarded. To achieve symmetry and maintain the simplicity of the analysis of variance, the first determination for the second bottle was also discarded and the calculations were made on the remaining six data.

One analyst formed a glass disc from two portions from each bottle of sample and counted the response of several elements or oxides from three exposures of each disc to X-rays. His data, therefore, had two variables of classification, the two bottles and the glass discs, and the error term in the analysis of variance is the error in the measurements of the discs.

One organization reported determinations by XRF on four portions from each of the three bottles of sample. This presented no problem in the analysis of variance because the abbreviation of the organization is asterisked to note the change in the degrees of freedom for error. However, rather than discard half the data when calculating best values, random numbers were used to determine which two of the four data for any bottle would be assigned to a first set of data and the remaining two data were assigned to the second set. Thus, two complete sets of data were available to be used for best values.

Some organizations reported fewer than the six determinations that would be required for the calculations of best values, and the averages of the five or fewer values were entered, followed by dashes for the standard deviations and the $F$ ratio. Another organization reported the necessary six determinations, but three analysts made two determinations each. As the data lacked the necessary symmetry to sort out the effects of the three analysts, the average of the six data was entered and the six data were also used for best values.

The tabulation below for the three samples shows the number of sets of data for which a significant (S) or not significant (NS) $F$ ratio was obtained when the calculated ratios were tested against the appropriate value for $F_{0.05}$.

\begin{tabular}{lrrrr}
\hline & W-2 & DNC-1 & BIR-1 & Total \\
\cline { 2 - 5 } NS - & 532 & 492 & 393 & 1417 \\
No variation ------- & 10 & 17 & 20 & 47 \\
No $F$ test ------- & 20 & 14 & 13 & 47 \\
S - & 23 & 30 & 40 & 93 \\
\cline { 2 - 5 }$\quad$ Total -- & 585 & 553 & 466 & 1604 \\
\hline
\end{tabular}

Eighty eight percent (1417) of the calculated $F$ ratios were found to be not significant (NS), and the constituent for each test may be said to be homogeneously distributed among the bottles of a sample; thus, the three samples are suitable for use as geochemical reference samples (GRS). The classification of "No variation" indicated that the determinations reported all had the same value and, therefore, a zero variance, whereas the classification of "No $F$ test" indicates that a zero, or approximately zero, bottle or error standard deviation was obtained during the analysis of variance. For the latter classification, the calculation of an $F$ ratio was impossible or inadvisable.

More than half of the 47 sets of data for which no variation was reported were for the minor oxides. Analysts might have reported the next uncertain digit in their data to prevent the occurrence of so many sample variances of zero. These zero variances tend to minimize the calculated homogeneous variances, which, in turn, may slightly inflate the Studentized ranges discussed in the next section.

\section{CALCULATIONS OF BEST VALUES}

Best values for the three samples were calculated in the same manner as those for the manganese nodule standards in U.S. Geological Survey Professional Paper 1155 (Flanagan and Gottfried, 1980). The method, briefly, is to find a homogeneous variance by Cochran's test and then to use the square root of this variance to Studentize the range of sets of means to determine which means belong to the same population. The method is briefly illustrated in example 1 of section 5.53 of Bennett and Franklin (1954).

The procedure for the homogeneous variance for the zirconium data for $\mathrm{W}-2$ is shown in table 7 . The sample variances, each with $n-1$ degrees of freedom (d.f.), were calculated for each set of six determinations reported; these variances are listed in increasing numerical order. The cumulative sums of these variances are given in the second column. The ratio of the largest variance to its matching cumulative sum of variances is then calculated successively down the column until the calculated ratio does not exceed the critical value for Cochran's test at some probability, $p$.

The ratio, $3.87 / 11.27=0.3434$, does not exceed the critical value of 0.5065 at $p=0.05$ and the homogeneous variance calculated from 11.27 , the cumulative sum of the first five variances, would have 25 degrees of freedom, five for each variance. I decided to accept the calculated ratio, 0.3802 , which is less than the critical value, 0.4225 , at $p=0.01$ so that 40 d.f. would be available. The acceptance of the ratio at $p=0.01$ and a homogeneous variance with 40 d.f. results in the slightly more stringent requirement of a lower critical value that the calculated Studentized range should not exceed. 
Table 7. Calculation of a homogeneous variance for $\mathrm{Zr}$ data in $\mathrm{W}-2$ $\left[s^{2}\right.$, laboratory variance, each with five degrees of freedom. L, largest laboratory variance. Methods: INAA, instrumental neutron activation analysis; OES, optical emission spectroscopy; SSMS, spark source mass spectroscopy; XRF, $X$-ray fluorescence spectroscopy.]

\begin{tabular}{lrrrrr}
\hline Method & \multicolumn{1}{c}{$\mathrm{s}^{2}$} & Sum $\mathrm{s}^{2}$ & $\mathrm{~L} /$ Sum & \multicolumn{2}{c}{$\begin{array}{c}\text { Critical Values for } \\
\text { Cochran's Test }\end{array}$} \\
\cline { 3 - 6 } & & & & \multicolumn{3}{c}{$\mathrm{p}=0.05$} & $\mathrm{p}=0.01$ \\
\hline SSMS & 323.15 & 658.06 & 0.4911 & 0.3029 & 0.3572 \\
OES & 222.67 & 334.91 & .6649 & .3286 & .3870 \\
OES & 42.67 & 112.24 & .3802 & .3595 & .4225 \\
OES & 30.30 & 69.57 & .4370 & .3974 & .4659 \\
XRF & 27.90 & 39.17 & .7123 & .4447 & .5195 \\
XRF & 3.87 & 11.27 & .3434 & .5065 & \\
INAA & 3.77 & 7.40 & & & \\
XRF & 1.73 & 3.63 & & & \\
XRF & 1.28 & 1.90 & & & \\
XRF & .62 & - & & & \\
\end{tabular}

The homogeneous variance is the average of the eight variances in the sum, 112.24 , or 14.03 . The square root of this variance, 3.74 , is the standard deviation of the means of six determinations with $40 \mathrm{~d}$.f. used to Studentize the range of the means of the several laboratories reporting data for $\mathrm{Zr}$.

The selection of a best value from among a seemingly heterogeneous group of means is essentially the process of determining which of the several means form a suitable group of nearest neighbors and then calculating the grand mean of the group. The laboratory means are listed in table 8 in increasing order from the least to the greatest mean and their sum is divided by their number, $\mathrm{n}$, to obtain a temporary grand mean, $\overline{\overline{\mathrm{x}}}$. The range of the means, 40.66 , is divided by the standard deviation, 3.74 , to obtain the Studentized range, 10.87. This far exceeds the critical value of the Studentized range (CSR), 4.74, in table 5.8 of Bennett and Franklin (1954) for $n=10$ means and 40 d.f. for the standard deviation. By inspection, the mean, 65.50 , is farther from the temporary grand mean than is 106.16 . The mean, 65.50 , is discarded and a new sum is obtained. The process is repeated until the Studentized range does not exceed the critical value, which decreases as the number of means decreases. The last temporary grand mean, 99.99 , rounded to 100.0 , is accepted as the best value for $\mathrm{Zr}$ in $\mathrm{W}-2$.

\section{BEST VALUES}

The best values for constituents of the three samples are given in table 9 as a grand mean, $\overline{\bar{x}}$, and the number of laboratory averages, $n$, included in the grand mean. The best estimates for W-1 from the 1972 compilation of data (Flanagan, 1976) are listed next to the
Table 8. Calculations for the best value, $\overline{\bar{x}}$, the grand mean, for $\mathrm{Zr}$ data in sample $\mathrm{W}-2$ by the Studentized range

[Abbreviations of methods as in Table $7, \bar{x}$, laboratory mean. $n$, number of laboratory means. $R$, range of laboratory means. SR, Studentized range. CSR, critical Studentized range. $\bar{x}_{L}$ and $\bar{x} S$, the largest and smallest laboratory means in the group. -, laboratory means no longer considered in the sequential tests, disc., discarded]

\begin{tabular}{lccccc}
\hline \multirow{2}{*}{ Method } & \multicolumn{5}{c}{ Laboratory means in the sequential tests } \\
\cline { 3 - 7 } INAA & 106.16 & 106.16 & 106.16 & 106.16 & 106.16 \\
XRF & 104.33 & 104.33 & 104.33 & 104.33 & 104.33 \\
XRF & 100.85 & 100.85 & 100.85 & 100.85 & 100.85 \\
SSMS & 97.97 & 97.97 & 97.97 & 97.97 & 97.97 \\
OES & 96.66 & 96.66 & 96.66 & 96.66 & 96.66 \\
XRF & 93.97 & 93.97 & 93.97 & 93.97 & 93.97 \\
XRF & 85.66 & 85.66 & 85.66 & 85.66 & - \\
OES & 82.00 & 82.00 & 82.00 & - & - \\
OES & 79.66 & 79.66 & - & - & - \\
XRF & 65.50 & - & - & - & - \\
& & & & & \\
Sum of $\bar{x}$ & 912.76 & 847.26 & 767.60 & 685.60 & 599.94 \\
$n$ & 10 & 9 & 8 & 7 & 6 \\
$\overline{\bar{x}}$ & 91.28 & 94.14 & 95.95 & 97.94 & 99.99 \\
$R$ & 40.66 & 26.50 & 24.16 & 20.50 & 12.19 \\
SR & 10.87 & 7.08 & 6.46 & 5.48 & 3.26 \\
CSR & 4.74 & 4.63 & 4.52 & 4.39 & 4.23 \\
$\bar{x}_{\mathrm{L}}-\overline{\bar{x}}$ & 14.88 & 12.02 & 10.21 & 8.22 & \\
$\overline{\bar{x}}-\bar{x} \bar{x}_{S}$ & 25.78 & 14.48 & 13.95 & 12.28 & \\
$\bar{x}$ disc. & 65.50 & 79.66 & 82.00 & 85.66 & \\
\hline & & & & & \\
\hline & & & & & \\
\hline
\end{tabular}

best values for W-2 to facilitate a direct comparison of the compositions of the samples. Many best values in table 9 are reported with extra significant digits. These may be useful in future calculations, but generally the last digit should be rounded when the samples are used for calibration.

The standard deviation of the mean, $s_{\bar{x}}$, and its associated degrees of freedom are listed, when available, for each sample and constituent. These standard deviations may be useful to future analysts who might wish to determine if their mean of six determinations may be considered as part of the same population from which best values were calculated. These values may change as more sets of six determinations become available and as the calculations are repeated after including new data.

Magnitudes and lower limits of estimation are entered for several elements because of insufficient data. The presence of a best value for which there is only a single mean should cause no problem.

There are several elements for which a mean is listed, followed by an $\mathrm{n}$ of 2 or 3 , but there is no estimate of the standard deviation and its associated degrees of freedom. Such estimates usually occur when the variances of two or three sets of six data differ significantly and there is no easy alternative other than to average the two or three means. 
Table 9. Best estimates of the compositions of samples $W-1, W-2$, DNC-1, and BIR-1

[The grand mean, $\overline{\bar{x}}$, and the standard deviation of the mean, $\mathrm{s}_{8}$, are in percent for $\mathrm{SiO}_{2}$ through $\mathrm{Fe}_{2} \mathrm{O}_{3} \mathrm{C}$, but are in parts per million for $\mathrm{Ag}$ through $\mathrm{Zr}$. $n$, number of laboratory means from which $\overline{\bar{x}}$ was calculated. $\mathrm{df}$, degrees of freedom. The number of laboratory variances from which the homogeneous variance, $s_{\bar{x}}^{2}$, was derived equals df/5. $\mathrm{Fe}_{2} \mathrm{O}_{3} \mathrm{~T}$, total $\mathrm{Fe}$ as $\mathrm{Fe}_{2} \mathrm{O}_{3}$. $\mathrm{Fe}_{2} \mathrm{O}_{3} \mathrm{C}$ was calculated from best values for $\mathrm{Fe}_{2} \mathrm{O}_{3}$ and $\mathrm{FeO}$. Data for $\mathrm{W}-1$ are from $\mathrm{Flanagan}$ (1976)]

$\underline{W-1}$ W-2 DNC-1 BIR-1 (1976)
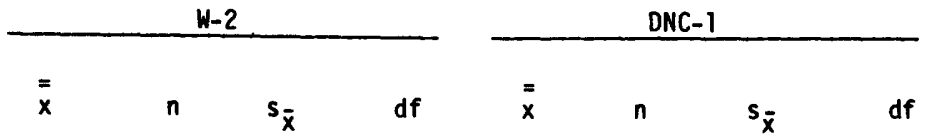

$\frac{\text { BIR-1 }}{\bar{x}} \quad n \quad s_{\bar{x}} \quad d f$

Percent

\begin{tabular}{|c|c|c|c|c|c|c|c|c|c|c|c|c|c|}
\hline $\mathrm{SiO}_{2}$ & 52,64 & 52.68 & 18 & 0.29 & 90 & 47.15 & 11 & 0.21 & 70 & 47.96 & 13 & 0.19 & 65 \\
\hline $\mathrm{Al}_{2} \mathrm{O}_{3}$ & 15.00 & 15.45 & 17 & .16 & 105 & 18.34 & 16 & .169 & 100 & 15.53 & 12 & .15 & 90 \\
\hline $\mathrm{Fe}_{2} \mathrm{O}_{3}$ & 1.40 & 1.53 & 6 & .087 & 35 & 1.79 & 4 & 107 & 35 & 2.06 & 5 & .104 & 35 \\
\hline $\mathrm{FeO}$ & 8.72 & 8.34 & 6 & .093 & 35 & 7.32 & 4 & .062 & 30 & 8.34 & 5 & .097 & 30 \\
\hline $\mathrm{MgO}$ & 6.62 & 6.37 & 13 & .058 & 30 & 10.13 & 15 & .112 & 90 & 9.70 & 10 & .079 & 70 \\
\hline $\mathrm{CaO}$ & 10.96 & 10.86 & 11 & .078 & 90 & 11.49 & 9 & .073 & 70 & 13.32 & 16 & .12 & 90 \\
\hline $\mathrm{Na}_{2} \mathrm{O}$ & 2.15 & 2.20 & 17 & .037 & 120 & 1.886 & 21 & .057 & 125 & 1.820 & 16 & .045 & 120 \\
\hline $\mathrm{k}_{2} \mathrm{O}$ & .64 & .626 & 20 & .012 & 95 & .234 & 15 & .009 & 85 & .030 & 11 & .003 & 60 \\
\hline $\mathrm{H}_{2} \mathrm{O}^{+}$ & .53 & .55 & 6 & .036 & 30 & .73 & 4 & .040 & 15 & .086 & 5 & .025 & 25 \\
\hline $\mathrm{H}_{2} \mathrm{O}^{-}$ & .16 & .25 & 3 & .018 & 15 & .29 & 3 & .047 & 15 & .078 & 2 & .016 & 15 \\
\hline $\mathrm{TiO}_{2}$ & 1.07 & 1.062 & 19 & .013 & 100 & .484 & 16 & .007 & 95 & .96 & 15 & .010 & 75 \\
\hline $\mathrm{P}_{2} \mathrm{O}_{5}$ & .14 & .141 & 18 & .116 & 80 & .070 & 9 & .005 & 55 & .021 & 4 & 1.0014 & 40 \\
\hline Mno & .17 & .167 & 20 & .004 & 105 & .148 & 14 & .003 & 75 & .175 & 13 & 1.003 & 75 \\
\hline $\mathrm{Fe}_{2} \mathrm{O}_{3} \mathrm{~T}$ & - & 10.83 & 25 & .208 & 120 & 9.972 & 23 & .153 & 110 & 11.29 & 20 & .125 & 80 \\
\hline $\mathrm{Fe}_{2} \mathrm{O}_{3} \mathrm{C}$ & 11.09 & 10.80 & - & - & - & 9.93 & - & - & - & 11.33 & - & - & - \\
\hline
\end{tabular}

Parts per million

\begin{tabular}{lccccccccccccc}
\hline Ag & .081 & .048 & 1 & - & - & .026 & 1 & - & - & .04 & 1 & - & - \\
As & 1.9 & 1.16 & 4 & .46 & 20 & .12 & 1 & - & - & 2.1 & 2 & - & - \\
B & 15 & 210 & - & - & - & 2.9 & - & - & - & 2.5 & - & - & - \\
Ba & 160 & 173.6 & 10 & 11.3 & 50 & 117.6 & 11 & 10.5 & 65 & 6.08 & 5 & 2.58 & 30 \\
Be & .8 & 1.14 & 2 & .10 & 10 & .96 & 3 & - & - & 2.56 & - & - & - \\
Cd & .15 & 3.10 & - & - & - & 3.09 & - & - & - & 3.17 & - & - & - \\
Ce & 23 & 23.37 & 10 & 1.47 & 65 & 9.14 & 8 & .77 & 50 & 1.62 & 4 & .44 & 30 \\
Cl & 200 & 2150 & - & - & - & 260 & - & - & - & 280 & - & - & - \\
Co & 47 & 43.15 & 21 & 2.11 & 110 & 56.75 & 18 & 2.19 & 115 & 51.58 & 18 & 1.88 & 100 \\
Cr & 114 & 91.51 & 19 & 4.45 & 100 & 270.1 & 12 & 8.48 & 95 & 372.5 & 7 & 8.25 & 75 \\
Cs & .9 & 1.01 & 5 & .16 & 20 & .44 & 2 & - & - & .43 & 2 & - & - \\
Cu & 110 & 106.2 & 10 & 4.88 & 60 & 99.7 & 7 & 2.64 & 45 & 124.7 & 11 & 3.71 & 55 \\
Dy & 4 & 3.6 & 2 & .82 & 15 & 3.0 & 3 & .81 & 15 & 3.7 & 3 & .98 & 15 \\
Er & 2.4 & 31.6 & - & - & - & 31.7 & - & - & - & 31.7 & - & - & - \\
Eu & 1.11 & 1.12 & 11 & .060 & 50 & .59 & 9 & .027 & 60 & .55 & 8 & .046 & 45 \\
F & 250 & 2180 & - & - & - & 2115 & - & - & - & 259 & - & - & - \\
Ga & 16 & 16.8 & 4 & .89 & 30 & 14.7 & 5 & .92 & 25 & 15.0 & 5 & 1.0 & 25 \\
Gd & 4 & 3.9 & 2 & - & - & 2.2 & 2 & - & - & 2.2 & 1 & - & - \\
Hf & 2.67 & 2.60 & 8 & .178 & 40 & 1.01 & 8 & .052 & 25 & .64 & 4 & .078 & 20 \\
Ho & .69 & .68 & 2 & .113 & 15 & .44 & 2 & - & - & 1.9 & 1 & - & - \\
La & 9.8 & 10.36 & 12 & .59 & 70 & 3.58 & 8 & .305 & 40 & .63 & 4 & .072 & 25 \\
Li & 14.5 & 9.56 & 6 & .54 & 30 & 5.24 & 6 & .29 & 25 & 3.62 & 4 & .16 & 20
\end{tabular}


Table 9. Best estimates of the compositions of samples $W-1, W-2$, DNC-1, and BIR-1 (cont.)

$W-1$

(1976)

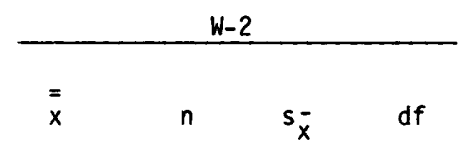

DNC-

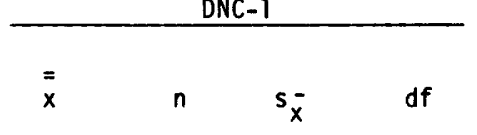

BIR-1

$\begin{array}{llll}\bar{x} & n & s_{\bar{x}} & \end{array}$

Parts per million

\begin{tabular}{|c|c|c|c|c|c|c|c|c|c|c|c|c|c|}
\hline $\mathrm{Lu}$ & 0.35 & 0.33 & 8 & 0.070 & 40 & 0.32 & 5 & 0.028 & 30 & 0.29 & 5 & 0.025 & 30 \\
\hline Mo & .57 & 2.7 & - & - & - & $\leq .2$ & - & - & - & 2.7 & - & - & - \\
\hline $\mathrm{Nb}$ & 9.5 & 6.75 & 4 & .42 & 25 & 3.19 & 4 & .42 & 20 & 2.26 & 4 & .42 & 15 \\
\hline $\mathrm{Nd}$ & 15 & 13.36 & 4 & 1.05 & 20 & 5.20 & 4 & .56 & 20 & 2.8 & 2 & - & - \\
\hline $\mathrm{Ni}$ & 76 & 70.4 & 10 & 2.46 & 50 & 247.0 & 12 & 11.78 & 75 & 166.4 & 9 & 5.88 & 55 \\
\hline $\mathrm{Pb}$ & 7.8 & 37.66 & - & - & - & 36.21 & - & - & - & 33.11 & - & - & - \\
\hline $\mathrm{Rb}$ & 21 & 20.9 & 11 & 1.06 & 55 & 4.7 & 5 & .37 & 40 & 2.2 & 2 & .28 & 10 \\
\hline$S$ & 123 & 463 & - & - & - & - & - & - & - & - & - & - & - \\
\hline Sb & 1.0 & .85 & 6 & .12 & 30 & .96 & 3 & .027 & 25 & .50 & 4 & .068 & 15 \\
\hline Sc & 35.1 & 35.7 & 9 & 1.06 & 100 & 31.4 & 10 & .98 & 55 & 43.4 & 8 & 1.13 & 40 \\
\hline $\mathrm{Sm}$ & 3.6 & 3.31 & 7 & .126 & 40 & 1.41 & 9 & .08 & 55 & 1.01 & 5 & .034 & 20 \\
\hline $\mathrm{Sr}$ & 190 & 192.0 & 8 & $T_{2.98}$ & 50 & 144.0 & 7 & 1.77 & 45 & 107.2 & 7 & 1.49 & 40 \\
\hline Ta & .50 & .52 & 6 & .053 & 25 & .10 & 2 & - & - & 2.07 & - & - & - \\
\hline Tb & .65 & .66 & 6 & .182 & 30 & .42 & 5 & .033 & 30 & .42 & 2 & .04 & 10 \\
\hline Th & 2.42 & 2.41 & 8 & .12 & 30 & .22 & 2 & - & - & $\leq .1$ & - & - & - \\
\hline TI & .11 & .16 & 1 & - & - & $<.1$ & - & - & - & $\overline{<.}$ & - & - & - \\
\hline Tm & .30 & .38 & 3 & .051 & 15 & .33 & 3 & .032 & 15 & .29 & 2 & .04 & 10 \\
\hline$u$ & .58 & .49 & 5 & .06 & 20 & $\leq .1$ & - & - & - & $\leq .1$ & - & - & - \\
\hline$v$ & 264 & 259.0 & 13 & 12.27 & 70 & 147.5 & 14 & 8.32 & 75 & $31 \overline{1.6}$ & 12 & 11.47 & 60 \\
\hline w & .5 & .26 & 1 & - & - & .19 & 1 & - & - & .22 & 1 & - & - \\
\hline$Y$ & 25 & 23.0 & 7 & 1.63 & 40 & 18.5 & 4 & .82 & 35 & 15.8 & 4 & .92 & 35 \\
\hline$Y b$ & 2.1 & 2.14 & 11 & .16 & 50 & 1.98 & 8 & .104 & 35 & 1.68 & 6 & .13 & 40 \\
\hline $\mathrm{Zn}$ & 86 & 79.6 & 10 & 2.28 & 55 & 70.1 & 10 & 2.36 & 60 & 69.6 & 6 & 2.02 & 45 \\
\hline $\mathrm{Zr}$ & 105 & 100.0 & 6 & 13.74 & 40 & 38.5 & 3 & .96 & 25 & 18.4 & 3 & 1.19 & 30 \\
\hline
\end{tabular}

\footnotetext{
$1 s_{\bar{x}}$ accepted at $p=0.01$

2 Magnitude

3 By isotope dilution mass spectrometry

4 By spark source mass spectrometry
}

Data by IDMS are collected in table 10. Most data are single determinations, but averages are noted by the number of determinations in parentheses. Data for erbium by this method have been entered as best values as they were the only data available.

\section{COMPOSITIONS OF W-1 AND W-2}

Although no petrographic work was done on the rock for sample $\mathrm{W}-2$, the original petrography by Chayes (1951) gives every expectation that the two samples should have the same composition. We thus have a unique opportunity to compare the compositions of the two samples, W-1 and its replacement W-2. The problem is how to compare the approximately 60 constituents that are common to both samples.

The technique of chi squared $\left(\chi^{2}\right)$ was used (Flanagan, 1964) to judge the analytical ability of a rock analyst by comparing his data for 14 constituents with the published means and standard deviations of all analyses. A variation of the same technique may be used to test the compositions of the two samples of the diabase. The tables of $\chi^{2}$ in Hald (1952) enable one to test as many as 100 constituents for the pair of samples with a choice of 21 probabilities.

The problem of making subjective judgements between a number of paired data is identical, except in 
Table 10. Data for several elements by isotope dilution mass spectrometry

IIn parts per miltion. Digits in parentheses are the number of replicates.]

\begin{tabular}{|c|c|c|c|}
\hline Element & $W-2$ & DNC-1 & BIR-1 \\
\hline$\overline{\mathrm{Ba}}$ & - & - & 6.8 \\
\hline $\mathrm{Cd}$ & $0.10(4)$ & $0.09(4)$ & $.17(4)$ \\
\hline $\mathrm{Pb}$ & 7.66 & 6.21 & 3.11 \\
\hline Dy & $3.2(2)$ & 2.0 & 2.0 \\
\hline Er & $1.6(2)$ & 1.7 & 1.7 \\
\hline $\mathrm{Eu}$ & $.8(2)$ & .6 & .5 \\
\hline $\mathrm{Gd}$ & 2.8 & 3.0 & 1.4 \\
\hline $\mathrm{Nd}$ & $10.5(2)$ & 2.6 & 4.6 \\
\hline Sm & $2.2(2)$ & 1.1 & 1.2 \\
\hline Yb & $2.8(2)$ & 3.5 & 1.5 \\
\hline
\end{tabular}

scope, whether we wish to compare the 14 constituents for a rock analysis or the large number of available comparisons in the summary table. The solution for the compositions of the two samples of the diabase is to reduce the differences between best values to some common base.

The solution for the present paired samples is more rigorous than the published example for G-1 (Flanagan, 1964). For the latter, I had the mean and standard deviation of all data published, but some data should have been discarded as no effort had been made to define the population of data. For the present pair of samples, I have calculated a homogeneous variance and have eliminated any laboratory means that could not be considered part of the population of data for W-2.

In calculations for the Studentized range, I used the standard deviation of the means of six determinations. From the general relation, $s_{\bar{x}}=s / \sqrt{n}$, we can multiply $s_{\bar{x}}$ by $\sqrt{6}$ to obtain an estimate of the population standard deviation. The calculations for chi squared are made in the form [(observed-expected)/s] ${ }^{2}$, where $s$ is the population standard deviation for $\mathrm{W}-2$, the expected values are the best values for $\mathrm{W}-2$, and the observed values are the 1972 estimates for W-1. The individual values for chi squared, each with 1 d.f., are listed in table 11.

I hypothesize that both samples have the same composition at a probability level for $\chi^{2}$ no higher than 95 percent. The sum of the individual contributions to $\chi^{2}$ in table 11 is 46.472 with 46 d.f. As this sum is far less than the critical value, 62.8 with 46 d.f., the compositions may be said to be the same at this probability. Reference to other probability levels in the table in Hald (1952) shows that the probability that $\chi^{2}$ is less than 47.8 is equal to 60 percent for 46 d.f. By rough linear interpolation between the value for 50 percent (45.3) and that for 60 percent (47.8), the compositions are the same at about the 55 percent level for the calculated $\chi^{2}$ of 46.47 .

The contributions of the individual constituents in table 11 may be used quantitatively to determine which
Table 11. Contributions of individual constituents to $x^{2}$

\begin{tabular}{|c|c|c|c|c|c|c|c|}
\hline oxide & $x^{2}$ & Element & $x^{2}$ & Element & $x^{2}$ & Element & $\chi^{2}$ \\
\hline $\mathrm{SiO}_{2}$ & 0.003 & As & 0.432 & Ho & 0.001 & $\mathrm{Ta}$ & 0.024 \\
\hline $\mathrm{Al}_{2} \mathrm{O}_{3}$ & 1.331 & $\mathrm{Ba}$ & .242 & La & .150 & Tb & .000 \\
\hline $\mathrm{Fe}_{2} \mathrm{O}_{3}$ & 1.378 & $\mathrm{Be}$ & 1.927 & Li & 13.953 & Th & .001 \\
\hline $\mathrm{FeO}$ & 2.778 & $\mathrm{Ce}$ & .011 & Lu & .014 & $\mathrm{Tm}$ & .410 \\
\hline MgO & 3.100 & $\mathrm{Co}$ & .555 & Nb & 7.148 & $\mathrm{U}$ & .297 \\
\hline $\mathrm{CaO}$ & .274 & $\mathrm{Cr}$ & 4.259 & $\mathrm{Nd}$ & .407 & $v$ & .028 \\
\hline $\mathrm{Na}_{2} \mathrm{O}$ & .305 & Cs & .079 & $\mathrm{Ni}$ & .864 & $Y$ & .251 \\
\hline $\mathrm{K}_{2} \mathrm{O}$ & .227 & $\mathrm{Cu}$ & 101 & $\mathrm{Rb}$ & .002 & $\mathrm{Yb}$ & .010 \\
\hline $\mathrm{TiO}_{2}$ & .063 & Dy & .040 & $\mathrm{Sb}$ & .260 & $\mathrm{Zn}$ & 1.314 \\
\hline $\mathrm{P}_{2} \mathrm{O}_{5}$ & .000 & Eu & .005 & Sc & .053 & $\underline{\mathrm{Zr}}$ & .298 \\
\hline Mno & .094 & $\mathrm{Ga}$ & .135 & $\mathrm{Sm}$ & .883 & & \\
\hline $\mathrm{Fe}_{2} \mathrm{O}_{3} \mathrm{~T}$ & .111 & Hf & 2.579 & $\mathrm{Sr}$ & .075 & Sum & 46.472 \\
\hline
\end{tabular}

constituents have the least and the greatest effect on $\chi^{2}$. Seven constituents having the greatest effect are listed below in order of decreasing contribution.

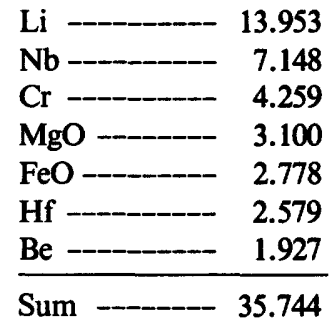

If we subtract the sum of these seven contributions and their degrees of freedom from the previous sum in table 11, we find that the total is now reduced to 10.728 with 39 d.f. Reentering the table of chi squared (Hald, 1952 ) for the value nearest to our 10.728 , we find that the probability that $\chi^{2}$ is less than 16.3 is equal to 0.05 percent for 39 d.f. Because of the low probability, we have reliable evidence that the compositions of the two samples are the same. A notable exception to the conclusion is shown in the $\mathrm{Hg}$ contents of the two samples (Flanagan and others, 1982); the $\mathrm{Hg}$ content of W-2 was determined to be $7.9 \mathrm{ppb}$ and the mercury contents of $\mathrm{W}-1$ were found to be in the range $89-210 \mathrm{ppb}$ by the same analysts and method.

Although it is recognized that best estimates for constituents of W-1 by Fleischer (1969) and Flanagan (1976) were essentially best guesses, a discussion of why some constituents were heavy contributors to $\chi^{2}$ seems worthwhile. We can consider the published data for W-1 from USGS Bulletin 1113 (Stevens and others, 1960) through the 1972 compilation of data (Flanagan, 1976). The data for $\mathrm{Li}$ in the five compilations show that both emission spectrographic and flame photometric data are converging on an estimate of $12 \mathrm{ppm}$. If all observations for the six data by AAS in the 1972 compilation had been reported, and if the number of observations were equal, we could estimate a best value for AAS by the same technique used for W-2. The process would undoubtedly result in a selection of 12,12.9, and 13 (average 12.6) as 
the group of nearest neighbors, rejecting the values of 17 , 20 , and $25 \mathrm{ppm}$. I can think of no reason why I should not have accepted the isotope dilution value of 12.6 , the basis of the recommended value for $\mathrm{Li}$ from Bulletin 1113 through the 1969 compilation.

We can then ask about the effect of the acceptance of $12.6 \mathrm{ppm}$ by Fleischer on the contribution to chi squared. If we calculate $[(12.6-9.6) / 0.54 \sqrt{6}]^{2}$, we obtain a value of 2.27 , which is slightly less than 2.71 , the critical value of $\chi^{2}$ at 90 percent. The laboratory means that survived the selection process are found between the dashed lines in table 12. The range of the eight laboratory means for $\mathrm{Li}$ in $\mathrm{W}-2$ does not include the average of 12.6 by isotope dilution for $\mathrm{W}-1$. The chi squared of 2.27 shows that we have a chance of about 10 percent of being incorrect if we conclude that the best values of $\mathrm{Li}$ for $\mathrm{W}-1$ and W-2 differ, but the difference seems more academic than real.

The original magnitude of $10 \mathrm{ppm}$ for $\mathrm{Nb}$ in $\mathrm{W}-1$ was based on data by one analyst and method, and this magnitude remained unchanged through the 1969 compilation. The 1972 data contained averages of 9.4 and 9.5 ppm determined spectrophotometrically in this laboratory, and we also found means of $5,6,10$, and 11.2 by $\mathrm{X}$-ray fluorescence. I accepted a best value of $9.5 \mathrm{ppm}$ either because or in spite of the fact that the analyst was a member of this laboratory.

Had I succumbed to an assumed initial impulse to take the mean of the six 1972 averages, I would have accepted $8.5 \mathrm{ppm} \mathrm{Nb}$ as the best value. This value would have reduced the contribution to chi squared by 4.34 , from the original 7.15 to 2.81 .

A magnitude of $120 \mathrm{ppm} \mathrm{Cr}$ had been accepted from Bulletin 1113 through the 1969 compilation. The ranges of the data in the 1972 compilation by optical emission $(25 \mathrm{ppm})$, neutron activation $(30 \mathrm{ppm})$, and atomic absorption $(24 \mathrm{ppm})$ covered a narrow span of 24-30 ppm, except for an obviously aberrant value of $174 \mathrm{ppm} \mathrm{Cr}$ by AAS. I probably averaged the means by these three techniques to arrive at a value of $114 \mathrm{ppm}$, and I see no reasonable alternative 10 years later. The $\mathrm{Cr}$ contents of W-1 and W-2 may indeed differ, but the best value of $92 \mathrm{ppm}$ in W-2 should not change radically.

The estimate of 8.74 percent $\mathrm{FeO}$ remained the same through the first three compilations. Fleischer (1969) changed his estimate to 8.72 percent, but no data for the 1972 compilation were sufficiently persuasive to indicate a change from 8.72 percent.

The literature contains material that may provoke speculation about the differing $\mathrm{FeO}$ contents of $\mathrm{W}-1$ and W-2. In his paper on the effect of metallic iron on ferrous iron determinations, Ritchie $(1968$, p. 1365) assumed a true $\mathrm{Fe}^{0}$ content for $\mathrm{W}-1$ of 0.05 percent, equivalent to 0.19 percent $\mathrm{FeO}$ by methods involving an oxidizing decomposition. Subtraction of this $\mathrm{FeO}$ from
Table 12. Laboratory averages for several constituents of W-2 [Means between dashes were used for best values. In parts per million, except $\mathrm{FeO}$ in percent.]

\begin{tabular}{|c|c|c|c|c|c|c|c|}
\hline $\mathrm{Li}$ & $\mathrm{Nb}$ & $\mathrm{Cr}$ & $\frac{c r}{(\operatorname{con} t)}$ & $\frac{\mathrm{Cr}}{(\operatorname{con} t)}$ & $\mathrm{FeO}$ & Hf & $\mathrm{Be}$ \\
\hline--- & 10.22 & 125.33 & 96.24 & 88.66 & $-\cdots$ & $-\cdots$ & 1.40 \\
\hline 10.17 & 9.38 & 125.00 & 94.17 & 87.83 & 8.637 & 2.73 & $\cdots$ \\
\hline 9.85 & 8.97 & 119.00 & 93.97 & 86.33 & 8.388 & 2.72 & 1.15 \\
\hline 9.78 & --- & 107.83 & 92.17 & 85.80 & 8.312 & 2.72 & 1.13 \\
\hline 9.50 & 7.50 & -..- & 92.00 & 85.17 & 8.283 & 2.72 & --- \\
\hline 9.18 & 7.29 & 100.83 & 91.50 & 80.66 & 8.200 & 2.57 & \\
\hline 8.87 & 5.87 & 98.33 & 90.60 & -... & 8.192 & 2.54 & \\
\hline--- & 5.46 & 97.50 & 90.17 & 74.67 & --- & 2.45 & \\
\hline 8.00 & $-\cdots$ & 97.50 & 89.33 & 54.78 & 7.908 & 2.35 & \\
\hline 6.83 & & & & & & $-\cdots$ & \\
\hline
\end{tabular}

the best value for $\mathrm{W}-1,8.72$, results in a value of 8.53 percent $\mathrm{FeO}$, a value much closer to the present best estimate for W-2. Had it occurred to me to reduce the 1972 values by the 0.19 percent $\mathrm{FeO}$, the contribution to $\chi^{2}$ would have been the more acceptable 0.158 rather than 2.778.

There is not much one can say about the Hf contents of the two samples. Values of $0.93,1.5,2$, and 2.0 were reported by spark-source mass spectrometry in earlier compilations and a value of 3.0 by neutron activation in 1969. Five data by neutron activation ranged from 2.2 to $3.4 \mathrm{ppm}$ in the 1972 compilation, and I undoubtedly averaged these for the estimate, $2.7 \mathrm{ppm}$, for $\mathrm{W}-1$. The data for $\mathrm{Hf}$ in $\mathrm{W}-2$ are all by neutron activation and it should not be surprising that the best value for W-2 is almost identical with that for $\mathrm{W}-1$. There seems little one can do to reduce this contribution to $\chi^{2}$.

The Be content of $\mathrm{W}-1$ was estimated by optical emission spectroscopy for each of the five compilations. Also, an isotope dilution value of $0.78 \mathrm{ppm} \mathrm{Be}$ in Bulletin 1113 was probably the basis of the estimate of $0.8 \mathrm{ppm}$ in the first four compilations. The data for 1972 were not sufficiently persuasive to indicate a change, and I retained the same estimate for 1972. Of the three means for $\mathrm{Be}$ in $\mathrm{W}-2$ (table 12), the value of 1.40 was rejected by the selection process, leaving two means to be averaged. No change that could be made would have reduced the contribution to $\chi^{2}$.

\section{THE RARE-EARTH ELEMENTS}

Best values for the rare-earth elements, normalized by the chondritic abundances of Haskin and others (1968), are plotted in figure 1. One may estimate the value of the plotted points from the number of laboratory averages included in the best values in summary table 9. The closed points for Er are IDMS data that were plotted to fill the void between Ho and Tm. 


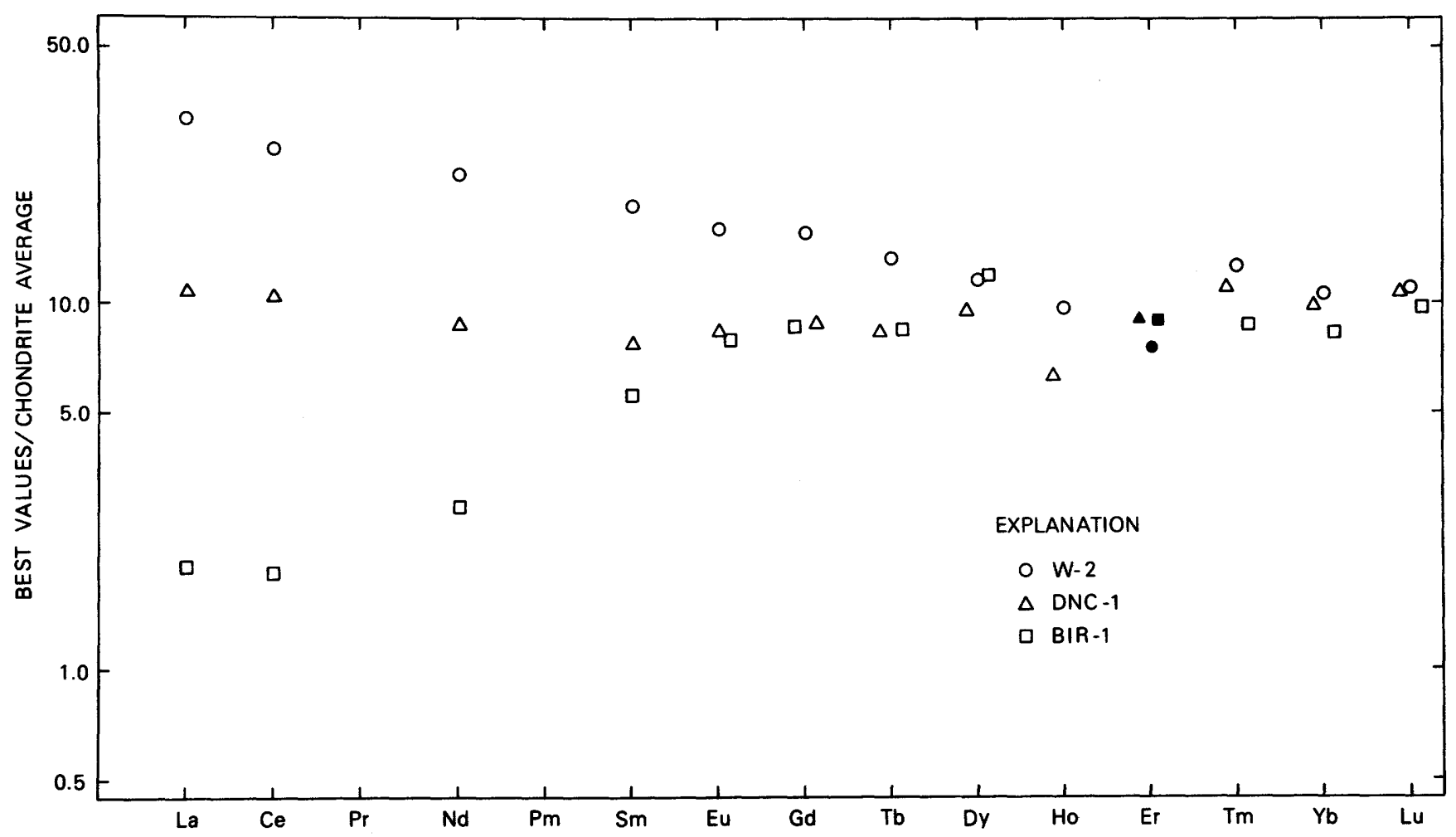

Figure 1. Best values for rare-earth elements normalized to chondritic abundances. (Solid symbols are single or duplicate determinations by IDMS. Symbols starting at Eu are offset to avoid plotting two or more symbols in the same space.)

Pairs and triads of some plotted points from Eu through $\mathrm{Lu}$ are offset to avoid plotting one symbol partly or entirely over another. These offsets will not materially affect the shape of the curve one obtains by connecting points for adjacent rare earths by straight lines.

The plotted points for $W-2$ show the shape of a curve that is familiar for $\mathrm{W}-1, \mathrm{BCR}-1$, and other normal basalts. The contents of the light rare-earth elements are low in the North Carolina dolerite, DNC-1, and are very low in the Icelandic basalt, BIR-1.

The shape of the plot for DNC-1 in figure 1 resembles the plot for the calc-alkaline island-arc basalt (sample 296B) from Talasea, New Britain, in figure 1 of Arth (1981). The rare-earth-element pattern for the Talasea basalt, for which all data are by IDMS, is much smoother than that for DNC-1, for which the plotted points are "best" values.

In contrast to the IDMS data in Arth (1981), most data available for calculating "best" values in the three samples were by INAA with an occasional set of data by XRF. Moreover, a calculated best value is only a group of the nearest neighbors of sets of data that can be considered as part of the population of all data for an element in a sample. More data by IDMS will result in smoother plots for the three samples.

\section{FUTURE ANALYTICAL. WORK}

Table 13 shows several other constituents, including cerium and four rare-earth elements, for which samples BIR-1, DNC-1, and W-2 furnish calibration points between the two ultramafic samples, DTS-1 and PCC-1, and the basalts, BHVO-1 and BCR-1. Except for $\mathrm{Li}, \mathrm{Rb}$, and $\mathrm{Sr}$ in BHVO-1, the trace element contents in table 13 decrease with decreasing $\mathrm{K}_{2} \mathrm{O}$ contents of the samples.

Although the best values in summary table 9 were obtained by a procedure which defines, where possible, the population of data for each constituent in each sample, the final values are estimates that depend upon the data submitted. Analysts using a method whose response is linear with concentration should check the validity of the best values for the elements in the five USGS mafic rocks.

The number of elements for which data are reported in table 9 is encouraging. More data will probably be reported because of the relatively large supply of sample powders that should be available for many years. In addition to data by IDMS for the rare-earth elements and for other elements useful in geochemical studies, analysts might, as a first approximation, examine the columns 
Table 13. Some trace element contents of USGS mafic and ul tramafic samples [In parts per million.]

\begin{tabular}{|c|c|c|c|c|c|c|c|}
\hline \multirow[b]{2}{*}{ Ref. } & \multirow{2}{*}{$\frac{\text { DTS-1 }}{(1)}$} & \multirow{2}{*}{$\frac{P C C-1}{(1)}$} & \multirow{2}{*}{$\underline{B I R-1}$} & DNC-1 & \multirow{2}{*}{$k-\frac{W-2}{k}$} & \multirow{2}{*}{$\frac{B H V O-1}{(2)}$} & \multirow{2}{*}{$\frac{B C R-1}{(1)}$} \\
\hline & & & & This & & & \\
\hline $\mathrm{Ba}$ & 2.4 & 1.2 & 6.1 & 117.6 & 173 & 142 & 675 \\
\hline$F$ & 15 & 15 & 59 & 115 & 180 & 380 & 470 \\
\hline Hf & .01 & .06 & .64 & 1.01 & 2.60 & 4.2 & 4.7 \\
\hline Li & 2 & 2 & 3.6 & 5.2 & 9.6 & 4.5 & 12.8 \\
\hline $\mathrm{Rb}$ & .05 & .06 & 2.2 & 4.7 & 21 & 10 & 46 \\
\hline$s r$ & .35 & .41 & 107 & 144 & 192 & 440 & 330 \\
\hline $\mathrm{Zr}$ & 3 & 7 & 18 & 38 & 100 & 180 & 190 \\
\hline La & .04 & .15 & .63 & 3.6 & 10.4 & 16.7 & 26 \\
\hline $\mathrm{Ce}$ & .06 & .09 & 1.6 & 9.1 & 23 & 41 & 54 \\
\hline Sm & .004 & .008 & 1.01 & 1.41 & 3.3 & 6.1 & 6.6 \\
\hline $\mathrm{Eu}$ & .001 & .002 & .55 & .59 & 1.12 & 2.0 & 1.94 \\
\hline Tb & .001 & .001 & .42 & .42 & .66 & 1.0 & 1.0 \\
\hline
\end{tabular}

1 Flanagan (1976)

2 Gladney and Goode (1981)

labeled " $n$ " in table 9 to determine those elements for which more data are necessary or desirable. For example, some elements were reported by only one analyst, others were reported as lower limits, and for several elements, including gold and the platinum group metals, no data were reported.

\section{REFERENCES}

Abbey, Sydney, 1980, Studies in "standard samples" for use in the general analysis of silicate rocks and minerals; Part 6, 1979 edition of "usable" values: Geological Survey of Canada Paper 80-14, 30 p.

Arth, J. G., 1981, Rare-earth element geochemistry of the island-arc volcanic rocks of Rabaul and Talasea, New Britain: Geological Society of America Bulletin, Part 1, v. 92, no. 11, p. 858-863.

Baedecker, P. A., Rowe, J. J., and Steinnes, Eiliv, 1977, Application of epithermal neutron activation in multielement analysis of silicate rocks employing both coaxial $\mathrm{Ge}(\mathrm{Li})$ and low energy photon detector systems: Journal of Radioanalytical Chemistry, v. 40, no. 1-2, p. 115-146.

Bastron, Harry, Barnett, P. R., and Murata, K. J., 1960, Method for the quantitative spectrochemical analysis of rocks, minerals, ores, and other materials by a powder $\mathrm{d}-\mathrm{c}$ arc technique: U.S. Geological Survey Bulletin 1084-G, p. 165-182.

Benedetti, A., Frache, R., Jaffrezic, H., Joron, J. L., Mazzucotelli, A., Meloni, S., and Oddone, M., 1977, Major, minor, and trace element data of ophiolite standard rock GOG-1: Ofioliti, v. 2, no. 1, p. 29-39.

Bennett, C. A., and Franklin, N. L., 1954, Statistical analysis in chemistry and the chemical industry: New York, Wiley, $724 \mathrm{p}$.
Borley, G. D., and Rogers, N., 1979, Comparison of rare-earth element data obtained by neutron activation analysis using international rock and multi-element solution standards: Geostandards Newsletter, v. 3, no. 1, p. 89-92.

Bouvier, J.-L., and Abbey, Sydney, 1977, Une amélioration de la méthode à "tige filetée" pour le dosage des alcalins rares dans les roches: Analusis, v. 5, no. 7, p. 332-334.

Bouvier, J. L., Sen Gupta, J. G., and Abbey, Sydney, 1972, Use of an "automatic sulphur titrator" in rock and mineral analysis; determination of sulphur, total carbon, carbonate and ferrous iron: Geological Survey of Canada Paper 72-31, $11 \mathrm{p}$.

Boy, M., and Mazzucotelli, A., 1976, Atomic absorption determination of silica in rocks and minerals; a review of direct and indirect techniques: Ofioliti, v. 1, no. 2, p. 127-133.

Casanova, L., Emiliani, F., Fanti, E. and Venturelli, G., 1968, Contributo alla conoscenza di nuovi standards silicatici naturali. Dosaggi di $\mathrm{SiO}_{2}, \mathrm{Al}_{2} \mathrm{O}_{3}, \mathrm{Fe}$ tot. (come $\mathrm{Fe}_{2} \mathrm{O}_{3}$ ), $\mathrm{CaO}, \mathrm{MgO}, \mathrm{TiO}_{2}, \mathrm{MnO}$ e relativi controlli di accuratezza: Ateneo Parmense, Segione 2, Acta Naturalia, v. 4, fasc. 3, p. 3-23.

Chayes, Felix, 1951, Modal analyses of the granite and diabase test rocks: U.S. Geological Survey Bulletin 980, p. 59-68.

Farzaneh, A., and Troll, G., 1977, Pyrohydrolysis for the rapid determination of small and large amounts of fluorine in fluorides, silicates, and rocks using an ion-selective electrode: Geochemical Journal [Japan], v. 11, no. 3, p. 177-181.

1978, Pyrohydrolysis for the rapid determination of chlorine traces in silicate and non-silicate minerals and rocks: Zeitschrift für analytische Chemie, v. 292 , no. 4, p. 293-295.

Flanagan, F. J., 1964, Judging the analytical ability of rock analysts by chi squared: U.S. Geological Survey Professional Paper 501-C, p. C157-C158.

1967, U.S. Geological Survey silicate rock standards: Geochimica et Cosmochimica Acta, v. 31, no. 3, p. 289-308.

1974, Reference samples for the earth sciences: Geochimica et Cosmochimica Acta, v. 38, no. 12, p. 1731-1744.

1976, 1972 compilation of data on USGS standards: U.S. Geological Survey Professional Paper 840, p. 131-183.

Flanagan, F. J., and Gottfried, David, 1980, USGS rock standards, III; Manganese-nodule reference samples USGSNod-A-1 and USGS-Nod-P-1: U.S. Geological Survey Professional Paper 1155, $39 \mathrm{p}$.

Flanagan, F. J., Moore, Roosevelt, and Aruscavage, P. J., 1982, Mercury in geologic reference samples: Geostandards Newsletter, v. 6, no. 1, p. 25-46.

Fleischer, Michael, 1969, U.S. Geological Survey standards-I. Additional data on rocks G-1 and W-1, 1965-1967: Geochimica et Cosmochimica Acta, v. 33, no. 1, p. 65-79.

Gladney, E. S., and Goode, W. E., 1981, Elemental concentrations in eight new United States Geological Survey rock standards; a review: Geostandards Newsletter, v. 5, no. 1, p. 31-64. 
Govindaraju, Kuppusami, Mevelle, Guy, and Chouard, Charles, 1976, Automated optical emission spectrochemical bulk analysis of silicate rocks with microwave plasma excitation: Analytical Chemistry, v. 48, no. 9, p. 1325-1331.

Hald, Anders, 1952, Statistical tables and formulas: New York, Wiley, 97 p.

Hancock, R. G. V., 1976, Low flux multielement instrumental neutron activation analysis in archaeometry: Analytical Chemistry, v. 48, no. 11, p. 1443-1445.

Harris, J. L., Schwarz, L. J., D'Angelo, W. M., and Flanagan, F. J., 1981, Additional analytical data for gabbro, GOG-1: Geostandards Newsletter, v. 5, no. 2, p. 125-128.

Haskin, L. A., Haskin, M. A., Frey, F. A., and Wildeman, T. R., 1968, Relative and absolute terrestrial abundances of the rare earths, in Ahrens, L. H., ed., Origin and distribution of the elements: New York, Pergamon, p. 889-912.

Hooper, P. R., and Atkins, Lynne, 1969, The preparation of fused samples in X-ray fluorescence analysis: Mineralogical Magazine, v. 37, no. 287, p. 409-413.

Kato, Toyoaki, Sato, Nobuyoshi, and Suzuki, Nobuo, 1977, Multielement analysis of deep-sea sediments by photon activation: Chemical Society of Japan Bulletin, v. 50, no. 8, p. 1930-1933.

Langmyhr, F. J., 1969, The reporting of analytical results for reference minerals and rocks: Geochimica et Cosmochimica Acta, v. 33, no. 12, p. 1561-1562.

Larsen, E. S., Jr., 1948, Batholith and associated rocks of Corona, Elsinore, and San Luis Rey quadrangles, southern California: Geological Society of America Memoir 29, $182 \mathrm{p}$.

Leake, B. E., and others, 1969, The chemical analysis of rock powders by automatic X-ray fluorescence: Chemical Geology, v. 5, no. 1, p. 7-86.

Ledger, E. B., Tieh, T. T., and Rowe, M. W., 1980, Delayedneutron activation determination of uranium in thirteen French rock reference samples: Geostandards Newsletter, v. 4 , no. 2 , p. 5-8.

Masumoto, Kazuyoshi, Kato, Toyoaki, and Suzuki, Nobuo, 1978, Activation yield curves of photonuclear reactions for multielement photon activation analysis: Nuclear Instruments and Methods, v. 157, no. 3, p. 567-577.

Maxwell, J. A., 1968, Rock and mineral analysis: New York, Wiley Interscience, $584 \mathrm{p}$.

Mazzucotelli, Ambrogio, Frache, Roberto, Dadone, Arduino, and Baffi, Franca, 1976, Ion-exchange separation and atomic absorption determination of fifteen major, minor, and trace elements in silicates: Talanta, v. 23, no. 11-12, p. 879-882.

Millard, H. T., Jr., 1976, Determination of uranium and thorium in USGS standard rocks by the delayed neutron technique: U.S. Geological Survey Professional Paper 840, p. 61-65.

Miller, F. S., 1937, Petrology of the San Marcos Gabbro, Southern California: Geological Society of America Bulletin, v. 48 , no. 10 , p. $1397-1425$.
Norrish, K., and Hutton, J. T., 1969, An accurate X-ray spectrographic method for the analysis of a wide range of geological samples: Geochimica et Cosmochimica Acta, v. 33, no. 4, p. 431-453.

Perrault, G., Hebert, P., and Kubat, V., 1974, MRG-1: un nouveau matériau de référence de gabbro à pyroxène et olivine du Mont-Royal, P. Q., Canada: Canadian Journal of Spectroscopy, v. 19, no. 2, p. 63-67.

Quintin, M., Martin, A., and de Kersabiec, A. M., 1978, Analyse de 47 échantillons géochimiques de référence par fluorescence-X $(\mathrm{Cu}, \mathrm{Ga}, \mathrm{Ni}, \mathrm{Zn})$ et par absorption atomique ( $\mathrm{Cu}, \mathrm{Ni}, \mathrm{Zn})$ : Geostandards Newsletter, v. 2, no. 2, p. 199-209.

Ragan, D. M., 1963, Emplacement of the Twin Sisters Dunite, Washington: American Journal of Science, v. 261, no. 6, p. 549-565.

Ragland, P. C., Rogers, J. J. W., and Justus, P. S., 1968, Origin and differentiation of Triassic dolerite magmas, North Carolina, USA: Contributions to Mineralogy and Petrology, v. 20, no. 1, p. 57-80.

Rantala, R. T. T., and Loring, D. H., 1973, New low-cost Teflon decomposition vessel: Atomic Absorption Newsletter, v. 12, p. 97-99.

1975, Multi-element analysis of silicate rocks and marine sediments by atomic absorption spectrophotometry: Atomic Absorption Newsletter, v. 14, p. 117-120.

Ritchie, J. A., 1968, Effect of metallic iron from grinding on ferrous iron determinations: Geochimica et Cosmochimica Acta, v. 32, no. 12, p. 1363-1366.

Rose, H. J., Jr., Adler, Isidore, and Flanagan, F. J., 1963, $\mathrm{X}$-ray fluorescence analysis of the light elements in rocks and minerals: Applied Spectroscopy, v. 17, no. 4, p. 81-85.

Rosman, K. J. R., and de Laeter, J. R., 1980, Mass spectrometric isotope dilution determination of cadmium in geochemical reference samples: Geostandards Newsletter, v. 4 , no. 1, p. 1-3.

Shapiro, Leonard, 1975, Rapid analysis of silicate, carbonate, and phosphate rocks-Revised edition: U.S. Geological Survey Bulletin 1401, $76 \mathrm{p}$.

Shapiro, Leonard, and Brannock, W. W., 1962, Rapid analysis of silicate, carbonate, and phosphate rocks: U.S. Geological Survey Bulletin 1144-A, 56 p.

Steele, T. W., 1978, A guide to the reporting of analytical results relating to the certification of geological reference materials: Geostandards Newsletter, v. 2, no. 1, p. 31-33.

Stevens, R. E., and others, 1960, Second report on a cooperative investigation of the composition of two silicate rocks: U.S. Geological Survey Bulletin 1113, 126 p.

Stevens, S. S., 1946, On the theory of scales of measurement: Science, v. 103, no. 2684 , p. 677-680.

Voldet, P., and Haerdi, W., 1978, Determination of rare-earth elements in rocks by neutron activation followed by highresolution X-ray spectrometry or $\gamma$-spectrometry: Analytica Chimica Acta, v. 97, no. 1, p. 185-189. 
Table 1. Analytical data for USCS-W-2

- $\mathrm{SiO}_{2}$ through $\mathrm{Pe}_{2} \mathrm{O}_{3} \mathrm{~T}$ in percent; trace elements in parts per million. Org.,'Meth., organization and method, Details of methods, where available, are given under the organization name at the end of cable 3 . A sel: of data by an organization whose abbreviation is preceded by an asterisk contains data or estimates explained under the organization name. P ratios noted only by an asterisk could not be calculated because of a zero mean square for bottles or error. Ns, not significant at the $95 \%$ fractile, $F_{0.05}(2,3)=9.55$. Allowable $F$ ratios for other degrees of freedom (df) and probabilities ( $p$ ) are given at the end of the table for calculated $F$ ratios not followed by Ns. Neg., negative bottle variance. $\mathrm{Fe}_{2} \mathrm{O}_{3} \mathrm{~T}$, total $\mathrm{Fe}$ as $\mathrm{Fe}_{2} \mathrm{O}_{3} \cdot 7$

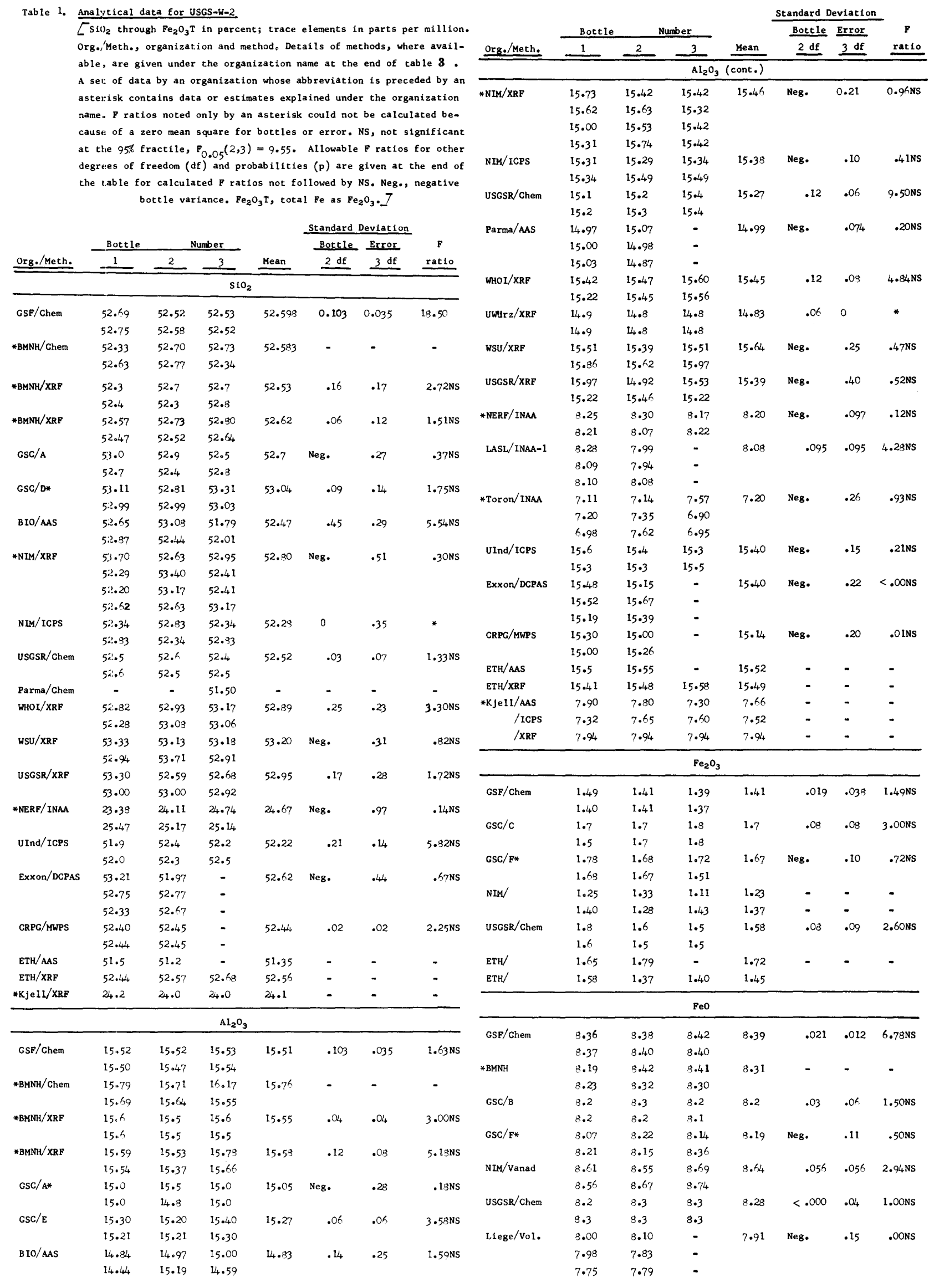




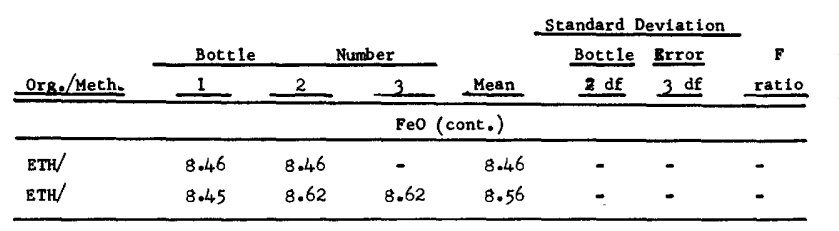

\begin{tabular}{llllllll}
\hline \multicolumn{7}{c}{ MgO } \\
\hline GSF/Chem & 6.63 & 6.63 & 6.64 & 6.63 & Neg. & .010 & $.50 \mathrm{NS}$ \\
& 6.64 & 6.62 & 6.62 & & & &
\end{tabular}

$\begin{array}{llllllll}\text { *BMMH } & 6.42 & 6.39 & 6.18 & 6.34 & - & - & - \\ & 6.54 & 6.23 & 6.31 & & & & \\ \text { *BMNH/XRF } & 6.62 & 6.57 & 6.64 & 6.58 & .012 & .047 & 1.22 \mathrm{NS}\end{array}$

$\begin{array}{llllllll}\text { *BMNH/XRF } & 6.62 & 6.57 & 6.64 & 6.58 & .012 & .047 & 1.22 \mathrm{NS} \\ & 6.56 & 6.50 & 6.57 & & & & \\ \text { *BMNH/XRF } & 6.41 & 6.40 & 6.48 & 6.44 & \text { Neg. } & .056 & .10 N S\end{array}$

$\begin{array}{llllllll}\text { *BMNH/XRF } & 6.41 & 6.40 & 6.48 & 6.44 & \text { Neg. } & .056 & .10 N S \\ & 6.44 & 6.50 & 6.39 & & & & \\ \text { GSC/A } & 6.52 & 6.33 & 6.37 & 6.39 & \mathrm{Neg} . & .09 & .19 \mathrm{NS}\end{array}$

$\begin{array}{llllllll} & 6.52 & 6.33 & 6.37 & 6.39 & \text { Neg. } & .09 & .19 \mathrm{NS} \\ \text { GSC/G } & 6.32 & 6.43 & 6.36 & & & & \\ & 6.44 & 6.36 & 6.37 & 6.43 & \mathrm{Neg} . & .08 & .05 \mathrm{NS}\end{array}$

$\begin{array}{llllllll} & 6.44 & 6.36 & 6.37 & 6.43 & \text { Neg. } & .08 & .05 \mathrm{NS} \\ \text { BIO/AAS } & 6.41 & 6.52 & 6.46 & & & & \\ & 6.27 & 6.27 & 6.27 & 6.24 & \mathrm{Neg} . & .05 & .70 \mathrm{NS} \\ & 6.23 & 6.15 & 6.27 & & & & \end{array}$

$\begin{array}{llllllll}* \mathrm{NIM} / \mathrm{XRF} & 6.23 & 6.15 & 6.27 & & & & \\ & 6.67 & 6.24 & 6.58 & 6.32 & \mathrm{Neg} . & .20 & .54 \mathrm{NS}\end{array}$

$\begin{array}{llllllll}* .67 & 6.24 & 6.58 & 6.32 & \text { Neg. } & .20 & .54 \mathrm{NS} \\ 6.24 & 6.33 & 6.01 & & & & \\ 6.27 & 6.30 & 6.22 & & & \\ 6.38 & 6.11 & 6.50 & & & & \end{array}$

$\begin{array}{llllllll}\text { NIM/ICPS } & 6.26 & 6.23 & 6.30 & 6.26 & \text { Neg. } & .038 & .03 \mathrm{NS} \\ & 6.25 & 6.29 & 6.23 & & & & \\ \text { USGSR/Chem } & 6.4 & 6.4 & 6.4 & 6.4 & - & - & - \\ & 6.4 & 6.4 & 6.4 & & & & \end{array}$

$\begin{array}{llllllll}\text { WHOI/XRF } & 6.67 & 6.59 & 6.61 & 6.60 & \text { Neg. } & .05 & .08 \mathrm{NS} \\ & 6.54 & 6.59 & 6.61 & & & & \\ \text { UWUIz / XRF } & 6.4 & 6.6 & 6.5 & 6.48 & .03 & .07 & 1.33 \mathrm{NS} \\ & 6.5 & 6.5 & 6.4 & & & & \end{array}$

\begin{tabular}{|c|c|c|c|c|c|c|c|}
\hline $\mathrm{WSU} / \mathrm{XRF}$ & 5.60 & 5.66 & 5.64 & 5.62 & Neg. & .08 & $55 \mathrm{NS}$ \\
\hline
\end{tabular}

$\begin{array}{lllllllll} & 5.71 & 5.49 & 5.65 & & & & \\ \text { USGSR/XRF } & 6.17 & 6.26 & 6.36 & 6.28 & .07 & .03 & 13.72\end{array}$

$\begin{array}{lllllllll} & 6.24 & 6.28 & 6.36 & & & & \\ \text { *Toron/INAA } & 3.32 & 3.53 & 3.25 & 3.42 & \text { Neg. } & .174 & .93 \mathrm{NS}\end{array}$

$\begin{array}{llllllll}\text { *Toron/INAA } & 3.32 & 3.53 & 3.25 & 3.42 & \text { Neg. } & .174 & .93 \mathrm{NS} \\ & 3.17 & 3.42 & 3.71 & & & & \\ \text { Tohok/IPAA } & 3.47 & 3.31 & 3.58 & & & & \\ & 6.18 & 5.97 & 6.43 & 6.21 & .16 & .08 & 8.18 \mathrm{NS} \\ \text { UInd/ICPS } & 6.30 & 6.08 & 6.30 & & & & \\ & 6.06 & 6.02 & 5.98 & 6.03 & \text { Neg. } & .04 & .80 \mathrm{NS} \\ & 5.99 & 6.09 & 6.02 & & & & \\ \text { Exxon/DCPAS } & 6.60 & 6.49 & - & 6.48 & .044 & .062 & 2.50 \mathrm{NS} \\ & 6.49 & 6.40 & - & & & & \\ & 6.46 & 6.42 & - & & & & \end{array}$

$\begin{array}{lllllllll}\text { GRPG/MWPS } & 6.15 & 6.10 & - & 6.11 & \text { Neg. } & .08 & .49 \mathrm{NS}\end{array}$

*USGSR/AAS $\quad \begin{array}{llllllll} & 3.70 & 3.62 & 3.72 & 3.70 & \text { Neg. } & .05 & .24 \text { NS }\end{array}$

$\begin{array}{llllllll} & 3.72 & 3.74 & 3.70 & & & & .05 \\ \text { ETH/AAS } & 6.24 & 6.26 & - & 6.25 & - & - & - \\ \text { ETH/XRF } & 6.20 & 6.26 & 6.25 & 6.24 & - & - & -\end{array}$

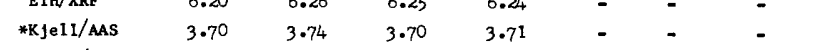

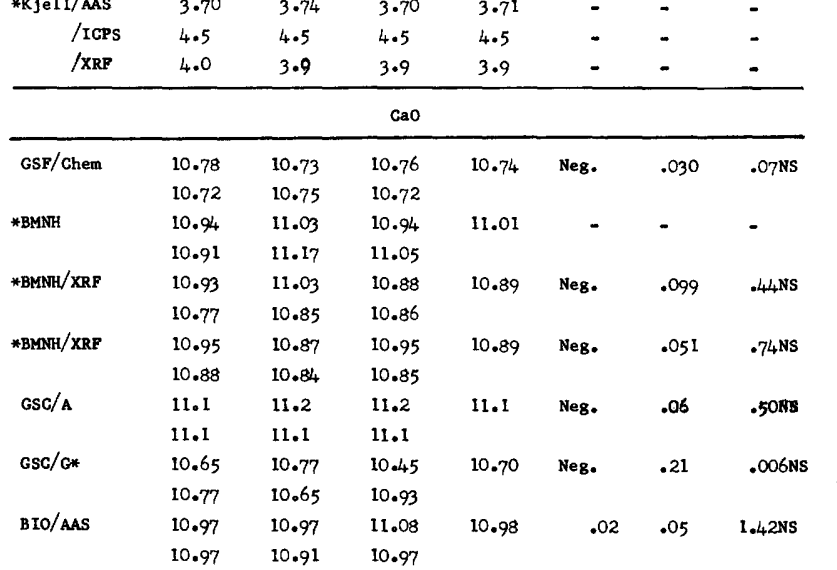

\begin{tabular}{|c|c|c|c|c|c|c|c|}
\hline \multirow[b]{3}{*}{ Org./Meth. } & \multirow{3}{*}{$\begin{array}{c}\text { Botcle } \\
1 \\
\end{array}$} & \multirow{2}{*}{\multicolumn{2}{|c|}{ Number }} & \multirow[b]{3}{*}{ Mean } & \multicolumn{3}{|c|}{ Standard Deviation } \\
\hline & & & & & \multicolumn{2}{|c|}{ Bottle Error } & \multirow{2}{*}{$\begin{array}{c}\mathbf{F} \\
\text { ratio } \\
\end{array}$} \\
\hline & & 2 & 3 & & $\underline{2 \mathrm{df}}$ & $3 \mathrm{df}$ & \\
\hline \multicolumn{8}{|c|}{$\mathrm{CaO}$ (cont.) } \\
\hline *NIM/XRF & 11.47 & 11.27 & 11.27 & 11.27 & Neg. & 0.12 & $0.78 \mathrm{NS}$ \\
\hline & 11.15 & 11.38 & 11.16 & & & & \\
\hline & 11.05 & 11.38 & 11.16 & & & & \\
\hline & 11.26 & 11.30 & 11.38 & & & & \\
\hline $\mathrm{NIM} / \mathrm{ICPS}$ & 11.30 & 11.25 & 11.25 & 11.26 & .035 & .020 & $7.00 \mathrm{Ns}$ \\
\hline & 11.30 & 11.25 & 11.20 & & & & \\
\hline USGSR/Chem & 10.7 & 10.6 & 10.5 & 10.55 & .06 & .09 & $1.80 \mathrm{Ns}$ \\
\hline & 10.6 & 10.4 & 10.5 & & & & \\
\hline WHOI/XRF & 11.14 & 11.17 & 11.12 & 11.15 & Neg. & .04 & $.11 \mathrm{Ns}$ \\
\hline & 11.14 & 11.11 & 11.19 & & & & \\
\hline UwUrz/XRF & 10.85 & 10.80 & 10.65 & 10.78 & .07 & .04 & $7.00 \mathrm{NS}$ \\
\hline & 10.85 & 10.80 & 10.75 & & & & \\
\hline $\mathrm{WSU} / \mathrm{XRF}$ & 10.13 & 10.17 & 10.22 & 10.19 & Neg. & .10 & $45 \mathrm{NS}$ \\
\hline & 10.27 & 10.30 & 10.06 & & & & \\
\hline USGSR/XRF & 11.33 & 11.47 & 11.28 & 11.33 & Neg. & .23 & $.38 \mathrm{NS}$ \\
\hline & 11.48 & 10.97 & 11.47 & & & & \\
\hline *Toron/INAA & 7.44 & 7.83 & 8.03 & 7.66 & .213 & .137 & 8.29 \\
\hline & 7.49 & 7.60 & $7 \cdot 82$ & & & & \\
\hline & 7.47 & 7.41 & 7.89 & & & & \\
\hline *HMI/INAA-W & 8.41 & 8.58 & - & 8.39 & Neg. & .47 & $.60 \mathrm{Ns}$ \\
\hline & 8.43 & $7 \cdot 50$ & - & & & & \\
\hline & 8.77 & 8.63 & - & & & & \\
\hline *HMI / INAA-B & 5.1 & 5.3 & - & 5.15 & Neg. & .32 & $.15 \mathrm{NS}$ \\
\hline & 5.2 & 4.6 & - & & & & \\
\hline & $5 \cdot 3$ & 5.4 & - & & & & \\
\hline *LASL/INAA-I & 8.4 & 8.5 & - & 8.12 & .082 & .426 & 1.IINS \\
\hline & 8.1 & 8.3 & - & & & & \\
\hline & $7 \cdot 3$ & 8.1 & - & & & & \\
\hline $\mathrm{LASL} / \mathrm{XRF}$ & 11.05 & 11.10 & - & 11.08 & - & - & - \\
\hline Tohok/IPAA & 10.73 & 10.51 & 10.86 & 10.72 & .11 & .07 & $5.79 \mathrm{NS}$ \\
\hline & 10.79 & 10.65 & 10.77 & & & & \\
\hline UInd/ICPS & 10.8 & 10.8 & 10.9 & 10.80 & 0 & .08 & * \\
\hline & 10.8 & 10.8 & 10.7 & & & & \\
\hline Exxon/DCPAS & 10.61 & 10.33 & - & 10.43 & .005 & .098 & $1.01 \mathrm{NS}$ \\
\hline & 10.42 & 10.44 & - & & & & \\
\hline & 10.37 & 10.39 & - & & & & \\
\hline GRPG/MWPS & 10.65 & 10.66 & - & 10.68 & Neg. & .05 & $.95 \mathrm{NS}$ \\
\hline & 10.67 & 10.75 & - & & & & \\
\hline ETH/AAS & 11.08 & 11.06 & - & 11.07 & - & - & - \\
\hline $\mathrm{ETH} / \mathrm{XRF}$ & 11.06 & 11.07 & $11.0_{4}$ & 11.06 & - & - & - \\
\hline *Kjell/AAS & 8.05 & 8.07 & 8.03 & 8.05 & - & - & - \\
\hline$/$ ICPS & 7.44 & 7.22 & $7 \cdot 36$ & $7 \cdot 34$ & - & - & - \\
\hline /XRF & 7.79 & 7.79 & 7.84 & 7.81 & - & $=$ & - \\
\hline & & & $\mathrm{Na}_{2} \mathrm{O}$ & & & & \\
\hline GSF/Chem & 2.10 & 2.11 & 2.11 & 2.11 & .006 & 0 & * \\
\hline & 2.10 & 2.11 & 2.11 & & & & \\
\hline GSC/A & 2.1 & 2.0 & 1.9 & 2.0 & .06 & .15 & $1.36 \mathrm{NS}$ \\
\hline & 1.8 & 2.2 & 1.8 & & & & \\
\hline GSC/G* & 2.25 & 2.25 & 2.27 & 2.26 & Neg. & .02 & $.39 \mathrm{NS}$ \\
\hline & 2.25 & 2.28 & 2.24 & & & & \\
\hline BIO/AAS & 2.18 & 2.18 & 2.20 & 2.18 & .01 & .01 & $1.44 \mathrm{NS}$ \\
\hline & 2.17 & 2.16 & 2.18 & & & & \\
\hline NWM/AAS & 2.24 & 2.24 & 2.22 & 2.24 & Neg. & .02 & .28NS \\
\hline & 2.25 & 2.26 & 2.26 & & & & \\
\hline USGSR/Chem & 2.3 & 2.2 & 2.2 & 2.22 & $<.000$ & .04 & $1.00 \mathrm{NS}$ \\
\hline & 2.2 & 2.2 & 2.2 & & & & \\
\hline Parma/F1Ph & 2.19 & 2.21 & - & 2.20 & .000 & .016 & $1.00 \mathrm{NS}$ \\
\hline & 2.20 & 2.22 & - & & & & \\
\hline & 2.18 & 2.18 & - & & & & \\
\hline WHOI/XRF & 1.96 & 1.98 & 1.90 & 1.92 & Neg. & .07 & $.02 \mathrm{NS}$ \\
\hline & 1.88 & 1.84 & 1.95 & & & & \\
\hline UWUIrz/XRF & 2.3 & 2.5 & 2.3 & 2.33 & .000 & .08 & $1.00 \mathrm{NS}$ \\
\hline & 2.3 & 2.3 & 2.3 & & & & \\
\hline WSU/XRF & 3.18 & 3.04 & 3.08 & 3.10 & Neg. & .12 & $.15 \mathrm{NS}$ \\
\hline & 2.97 & 3.23 & 3.09 & & & & \\
\hline USGSR/XRF & 2.05 & 2.14 & 2.14 & 2.11 & Neg. & .03 & $.96 \mathrm{NS}$ \\
\hline & 2.12 & 2.11 & 2.11 & & & & \\
\hline *Tor on/ INAA & 1.42 & 1.39 & 1.42 & 1.43 & Neg. & .033 & $.2 \mathrm{gNS}$ \\
\hline & 1.42 & 1.50 & 1.44 & & & & \\
\hline & 1.42 & 1.43 & 1.44 & & & & \\
\hline
\end{tabular}




\begin{tabular}{|c|c|c|c|c|c|c|c|}
\hline \multirow[b]{3}{*}{ Orge/Metho } & \multirow{3}{*}{ Bottle } & \multirow{2}{*}{\multicolumn{2}{|c|}{ Number }} & \multirow[b]{3}{*}{ Mean } & \multicolumn{2}{|c|}{ Standard Deviation } & \multirow{3}{*}{$\begin{array}{r}F \\
\text { ratio } \\
\end{array}$} \\
\hline & & & & & \multicolumn{2}{|c|}{ Bottle Error } & \\
\hline & & 2 & 3 & & $2 \mathrm{df}$ & $3 d f$ & \\
\hline \multicolumn{8}{|c|}{$\mathrm{K}_{2} \mathrm{O}$ (cont.) } \\
\hline \multirow[t]{3}{*}{ *HMI/INAA-B } & 0.47 & 0.53 & - & 0.49 & Neg. & 0.32 & $0.03 \mathrm{NS}$ \\
\hline & .47 & .42 & + & & & & \\
\hline & .52 & .53 & & & & & \\
\hline \multirow[t]{2}{*}{ *NERE/INAA } & .53 & - & .53 & .54 & - & - & - \\
\hline & - & .55 & .55 & & & & \\
\hline LASL/XRF & .52 & .54 & - & .53 & - & - & - \\
\hline UInd/ICPS & .64 & .61 & .59 & .61 & Neg. & .019 & $.62 \mathrm{NS}$ \\
\hline & .60 & .62 & .61 & & & & \\
\hline Exxon/DCPAS & .66 & .61 & - & .64 & Neg. & .019 & $.43 \mathrm{NS}$ \\
\hline & .63 & .65 & - & & & & \\
\hline & .63 & .63 & - & & & & \\
\hline CRPG/MWPS & .63 & .63 & - & .63 & .005 & .007 & $2.00 \mathrm{NS}$ \\
\hline & .64 & .62 & - & & & & \\
\hline USGSM/F1Ph-1 & .643 & .634 & .634 & .635 & Neg. & .0047 & $.56 \mathrm{NS}$ \\
\hline & .632 & .631 & .636 & & & & \\
\hline USGSM/F1Ph-2 & .638 & .616 & .629 & .625 & Neg. & .0089 & $.48 \mathrm{NS}$ \\
\hline & .618 & .624 & .625 & & & & \\
\hline *USGSR/AAS-1 & .51 & .53 & .50 & .51 & .004 & .009 & $1.40 \mathrm{NS}$ \\
\hline & .51 & .51 & .51 & & & & \\
\hline ETH/AAS & .66 & .68 & - & .67 & - & - & - \\
\hline $\mathrm{ETH} / \mathrm{XRF}$ & .60 & .60 & .61 & .60 & - & - & - \\
\hline$* \mathrm{KJel} / \mathrm{XRF}$ & .56 & .56 & .56 & .56 & - & - & - \\
\hline & & & $\mathrm{H}_{2} \mathrm{O}^{+}$ & & & & \\
\hline GSF/Grav & .56 & .56 & .60 & .58 & .017 & .009 & $7.79 \mathrm{NS}$ \\
\hline & .56 & .58 & .59 & & & & \\
\hline *BMNH/Grav & .60 & .58 & .57 & .59 & - & - & - \\
\hline & .60 & .62 & .57 & & & & \\
\hline $\mathrm{Gsc} / 2$ & .52 & .44 & .46 & .49 & Neg. & .04 & $.63 \mathrm{NS}$ \\
\hline & .50 & .50 & .52 & & & & \\
\hline $\mathrm{GSC} / \mathrm{Y}^{*}$ & .52 & .54 & .56 & .52 & Neg. & .02 & $.78 \mathrm{NS}$ \\
\hline & .50 & .50 & .52 & & & & \\
\hline USGSR/Chem & .48 & .58 & .60 & .57 & .02 & $.0_{4}$ & $1.56 \mathrm{NS}$ \\
\hline & .58 & .58 & .60 & & & & \\
\hline Liege/Grav & .55 & .49 & - & .54 & 0 & .06 & * \\
\hline & .49 & $.5 \mathrm{I}$ & - & & & & \\
\hline & .59 & .63 & - & & & & \\
\hline & & & $\mathrm{H}_{2} \mathrm{O}^{-}$ & & & & \\
\hline *BMNH/Grav & .20 & .22 & .23 & .22 & - & - & - \\
\hline & .20 & .24 & .24 & & & & \\
\hline $\mathrm{Gsc} / \mathrm{b}$ & .18 & .16 & .14 & .18 & Neg. & .02 & $.78 \mathrm{NS}$ \\
\hline & .20 & .20 & .18 & & & & \\
\hline USGSR/Chem & .30 & .29 & .27 & .28 & .013 & .004 & 21.00 \\
\hline & .29 & .29 & .27 & & & & \\
\hline Liege/Grav & .35 & .29 & - & .25 & Neg. & .08 & $.52 \mathrm{NS}$ \\
\hline & .29 & .24 & - & & & & \\
\hline & .18 & .15 & - & & & & \\
\hline & & & $\mathrm{TiO}_{2}$ & & & & \\
\hline GSF/Golor & 1.04 & 1.04 & 1.02 & 1.04 & $<.000$ & .008 & $1.00 \mathrm{NS}$ \\
\hline & 1.04 & 1.04 & 1.04 & & & & \\
\hline *BMNH/Color & 1.10 & 1.10 & 1.06 & 1.08 & - & - & - \\
\hline & 1.09 & 1.02 & 1.10 & & & & \\
\hline \#BMNH/XRF & 1.05 & 1.06 & 1.04 & $1.0_{4}$ & Neg. & .009 & $.60 \mathrm{NS}$ \\
\hline & 1.04 & 1.04 & 1.04 & & & & \\
\hline *BMNH/XRF & 1.043 & 1.050 & 1.055 & 1.051 & Neg. & .007 & $.01 \mathrm{NS}$ \\
\hline & 1.059 & 1.051 & 1.048 & & & & \\
\hline $\mathrm{Gsc} / \mathrm{A}$ & 1.08 & 1.09 & 1.10 & 1.08 & Neg. & .01 & .7 ONS \\
\hline & 1.07 & 1.09 & 1.07 & & & & \\
\hline $\mathrm{Gsc} / \mathrm{H}$ & 1.07 & 1.11 & 1.10 & 1.09 & .012 & .014 & $2.54 \mathrm{NS}$ \\
\hline & 1.08 & 1.10 & 1.07 & & & & \\
\hline BIO/AAS & 1.05 & 1.05 & 1.07 & 1.06 & 0 & .01 & * \\
\hline & 1.07 & 1.07 & 1.05 & & & & \\
\hline *NIM/XRF & 1.14 & 1.11 & 1.13 & 1.10 & Neg. & .04 & .89 NS \\
\hline & 1.10 & 1.11 & 1.09 & & & & \\
\hline & 1.07 & 1.11 & 1.09 & & & & \\
\hline & 1.10 & 1.10 & 1.09 & & & & \\
\hline $\mathrm{NIM} / \mathrm{ICPS}$ & 1.02 & 1.02 & 1.03 & 1.03 & Neg. & .012 & $.11 \mathrm{NS}$ \\
\hline & 1.04 & 1.04 & 1.04 & & & & \\
\hline
\end{tabular}


Table 1. Analytical data for USGS-W-2 (cont.)

\begin{tabular}{|c|c|c|c|c|c|c|c|c|c|c|c|c|c|c|c|}
\hline \multirow{4}{*}{ Org./Meth. } & \multirow{4}{*}{$\frac{1}{1}$} & \multirow{3}{*}{\multicolumn{2}{|c|}{ Number }} & \multirow[b]{4}{*}{ Mean } & & & & & \multirow{3}{*}{$\begin{array}{l}\text { Bottle } \\
1 \\
\end{array}$} & \multirow{2}{*}{\multicolumn{2}{|c|}{ Number }} & & & & \\
\hline & & & & & & & & & & & & & Bottle & Error & $\mathbf{F}$ \\
\hline & & & & & $\frac{\text { tandard D }}{\text { Bott le }}$ & $\frac{\text { Elation }}{\text { Error }}$ & $\mathbf{F}$ & Org./Meth. & & 2 & 3 & Mean & $2 \mathrm{df}$ & $3 \mathrm{df}$ & ratio \\
\hline & & 2 & 3 & & $2 \mathrm{df}$ & $3 \mathrm{df}$ & ratio & & & & $\mathrm{P}_{2} \mathrm{O}_{5}($ & cont.) & & & \\
\hline & & & $\mathrm{TiO}_{2}$ & (cont.) & & & & ETV/AAS & 0.17 & 0.17 & - & 0.17 & - & - & - \\
\hline USGSR/Chem & 1.1 & 1.1 & 1.1 & 1.1 & - & - & - & $\mathrm{ETH} / \mathrm{XRF}$ &.$u_{4}$ & .14 & .13 & .14 & - & $=$ & - \\
\hline & 1.1 & 1.1 & 1.1 & & & & & & & & Mno & & & & \\
\hline Parma/Color & 1.093 & 1.093 & - & 1.084 & 0 & .010 & * & GSE/Color & .16 & .16 & .16 & .16 & - & - & - \\
\hline & 1.081 & 1.089 & - & & & & & & .16 & .16 & .16 & & & & \\
\hline & 1.078 & 1.070 & - & & & & & BMNH/Color & .163 & - & .162 & .162 & - & - & - \\
\hline WHOI/XRF & 1.09 & 1.08 & 1.08 & 1.08 & .12 & .08 & $4 \cdot 84 \mathrm{NS}$ & *BMNH/XRF & .17 & .17 & .17 & .17 & - & - & - \\
\hline & 1.08 & 1.08 & 1.08 & & & & & & .17 & .17 & .17 & & & & \\
\hline UWUr z/XRF & $\begin{array}{l}1.08 \\
1.08\end{array}$ & $\begin{array}{l}1.06 \\
1.06\end{array}$ & $\begin{array}{l}1.06 \\
1.06\end{array}$ & 1.07 & .012 & .000 & * & *BMNH/XRF & .167 & .168 & .166 & .168 & .001 & .001 & $4.00 \mathrm{NS}$ \\
\hline $\mathrm{WSU} / \mathrm{XRF}$ & 1.03 & 1.03 & 1.05 & $1 . \alpha_{4}$ & Neg. & .02 & $.04 \mathrm{NS}$ & GSC/A* & $\begin{array}{l}.168 \\
.17\end{array}$ & $\begin{array}{l}.169 \\
.17\end{array}$ & $\begin{array}{l}.167 \\
.17\end{array}$ & .17 & $<.000$ & .004 & $1.00 \mathrm{NS}$ \\
\hline & 1.06 & 1.06 & 1.03 & & & & & & .17 & .17 & .16 & & & & \\
\hline USGSR/XRF & 1.07 & 1.05 & 1.06 & 1.06 & .007 & $.0 \alpha_{4}$ & $7.00 \mathrm{NS}$ & $\mathrm{GSC} / \mathrm{G} *$ & .17 & .17 & .17 & .17 & - & - & - \\
\hline *LASL/INAA-I & 1.06 & 1.05 & & & & & & & .17 & .17 & .17 & & & & \\
\hline *LASL/INAA-1 & $\begin{array}{l}.60 \\
.64\end{array}$ & $\begin{array}{l}.50 \\
.58\end{array}$ & - & .605 & Neg. & .023 & $.27 \mathrm{NS}$ & $\mathrm{GSC} / \mathrm{N}$ & .22 & .23 & .22 & .22 & Neg. & .009 & .20NS \\
\hline & .59 & .62 & - & & & & & & .23 & .21 & .22 & & & & \\
\hline LASL/XRF & 1.00 & .99 & - & 1.00 & - & - & - & *NIM/ $/$ RF & .16 & .13 & .26 & .167 & Neg. & .050 & $.46 \mathrm{NS}$ \\
\hline Tohok/IPAA & 1.07 & 1.05 & $1 . O_{4}$ & 1.06 & Neg. & .02 & $.75 \mathrm{NS}$ & & .15 & .16 & .14 & & & & \\
\hline & 1.07 & 1.05 & 1.08 & & & & & & .19 & .19 & .21 & & & & \\
\hline UInd/ICPS & 1.05 & 1.04 & 1.06 & 1.05 & .007 & $.0 \alpha_{4}$ & $7.00 \mathrm{NS}$ & & .09 & .21 & .11 & & & & \\
\hline & 1.05 & $1.0_{4}$ & 1.05 & & & & & NIM/AAS & .17 & .17 & .16 & .168 & $<.000$ & $.00 \alpha_{4}$ & $1.00 \mathrm{NS}$ \\
\hline Exxon/DCPAS & 1.12 & 1.08 & - & 1.09 & Neg. & .018 & $.20 \mathrm{NS}$ & & .17 & .17 & .17 & & & & \\
\hline & 1.09 & 1.09 & - & & & & & NDM/ICPS & .16 & .16 & .16 & .16 & - & - & - \\
\hline & 1.07 & 1.09 & - & & & & & & .16 & .16 & .16 & & & & \\
\hline CRPG/MWPS & 1.13 & 1.4 & - & 1.14 & 0 & .007 & * & USGSR/Chem & .19 & .21 & .21 & .202 & Neg. & $.0 \mathrm{I}$ & .IINS \\
\hline & 1.4 & 1.13 & - & & & & & & .21 & .19 & .20 & & & & \\
\hline ETH/AAS & 1.07 & 1.07 & - & 1.07 & - & - & - & WHOI/XRF & .172 & .168 & .175 & .172 & .002 & .002 & $2.45 \mathrm{NS}$ \\
\hline $\mathrm{ETH} / \mathrm{XRF}$ & 1.07 & 1.07 & 1.07 & 1.07 & - & - & - & & .168 & .172 & .174 & & & & \\
\hline *Kjell/ICPS & .64 & .66 & .64 & .65 & - & - & - & Uwurz/XRF & .16 & .16 & .16 & .16 & - & - & - \\
\hline /OEs & .46 & .45 & .45 & .45 & - & - & - & & $.16^{\prime}$ & .16 & .16 & & & & \\
\hline /XRE & .63 & .62 & .63 & .63 & - & - & - & $\mathrm{WSU} / \mathrm{XRF}$ & .16 & .17 & .16 & .165 & $.0 \alpha_{4}$ & .004 & $3.00 \mathrm{NS}$ \\
\hline & & & $\mathrm{P}_{2} \mathrm{O}_{5}$ & & & & & & .17 & .17 & .16 & & & & \\
\hline $\operatorname{csp} / \mathrm{col}$ or & & & & 11 & & & & USGSR/XRF & $\begin{array}{l}.17 \\
.17\end{array}$ & $\begin{array}{l}.17 \\
.17\end{array}$ & $\begin{array}{l}.16 \\
.17\end{array}$ & .168 & .000 & .004 & 1.0ONS \\
\hline GSE/Color & $\begin{array}{l}.11 \\
.11\end{array}$ & $\begin{array}{l}.11 \\
.11\end{array}$ & $\begin{array}{l}.11 \\
.11\end{array}$ & $\cdot 11$ & - & - & - & Tohok/IPAA & .161 & .155 & .169 & .163 & .002 & .005 & I.22NS \\
\hline *BMNH/Color & .15 & .02 & .19 & .13 & - & - & - & & .163 & .166 & .166 & & & & \\
\hline & .11 & .18 & .12 & & & & & UInd/ICPS & .15 & .15 & .16 & .153 & Neg. & .006 & $.50 \mathrm{NS}$ \\
\hline *BMNH/XRF & .12 & .12 & .11 & .12 & $<.000$ & .004 & $1.00 \mathrm{NS}$ & & .16 & .15 & .15 & & & & \\
\hline & .12 & .12 & .12 & & & & & Exxon/DCPAS & .17 & .17 & - & .17 & - & - & - \\
\hline \#BMNH/XRF & .131 & .131 & .134 & .133 & .001 & .002 & $1.76 \mathrm{NS}$ & & .17 & .17 & - & & & & \\
\hline & .135 & .132 & .136 & & & & & & .17 & .17 & - & & & & \\
\hline $\mathrm{GSC} / \mathrm{A}$ & .13 & .12 & .10 & .12 & Neg. & .019 & $.43 \mathrm{NS}$ & ETH/AAS & .164 & .166 & - & .165 & - & - & - \\
\hline & .09 & .13 & .12 & & & & & $\mathrm{ETH} / \mathrm{XRF}$ & .16 & .17 & .17 & .17 & - & - & - \\
\hline $\mathrm{Gsc} / \mathrm{J}^{*}$ & .13 & .13 & .13 & .13 & - & - & - & *Kjell/AAS & .1200 & .1230 & .1220 & .1217 & - & - & - \\
\hline & .13 & .13 & .13 & & & & & /ICPS & .1420 & .1428 & .1380 & .1409 & - & - & - \\
\hline *NIM/XRF & .17 & .19 & .18 & .19 & Neg. & .018 & $.34 \mathrm{NS}$ & & & & $\mathrm{CO}_{2}$ & & & & \\
\hline & .23 & .20 & .20 & & & & & & & & & & & & \\
\hline & .17 & .21 & .20 & & & & & *BMNH & .09 & .19 & .06 & .085 & - & - & - \\
\hline & .19 & .20 & .19 & & & & & & .05 & .06 & .06 & & & & \\
\hline USGSR/Chem & .15 & .14 & .15 & .148 & .003 & .007 & $1.33 \mathrm{NS}$ & $\mathrm{GSC} / \mathrm{a}$ & .0 & .0 & .0 & $<.1$ & - & - & - \\
\hline & .14 & .15 & .16 & & & & & & .0 & .1 & .0 & & & & \\
\hline Parma/Color & .13 & .13 & - & .132 & .006 & .006 & $4.50 \mathrm{NS}$ & $\mathrm{GSC} / \mathrm{Y} *$ & $.0_{4}$ & .06 & .05 & .06 & .003 & .01 & $1.17 \mathrm{NS}$ \\
\hline & .14 & .12 & - & & & & & & .06 & .07 & .06 & & & & \\
\hline & $\cdot 14$ & .13 & - & & & & & USGS/Chem & .02 & .02 & .02 & .02 & - & - & - \\
\hline WHOI/XRF & .47 & .150 & $\cdot u_{4} 2$ & $\cdot 146$ & .005 & .006 & $2.51 \mathrm{NS}$ & & .02 & .02 & .02 & & & & \\
\hline & .159 & .443 & .127 & & & & & & & & $\mathrm{Cl}$ & & & & \\
\hline UWUrz/XRF & .12 & .10 & $\cdot v_{4}$ & .125 & $.00 \mathrm{~K}$ & .017 & $1.24 \mathrm{NS}$ & & & & & & & & \\
\hline & .11 & $\cdot 1 / 4$ & .14 & & & & & $\mathrm{GSC} / \mathrm{L}$ & .04 & .02 & .02 & .02 & Neg. & .01 & $.36 \mathrm{NS}$ \\
\hline WSU/XRF & .12 & .13 & .13 & .128 & $<.000$ & .004 & $1.00 \mathrm{NS}$ & & .01 & .01 & .01 & & & & \\
\hline USGSR/XRF & $\begin{array}{r}.13 \\
.15\end{array}$ & .13 & .13 & & Neg. & .000 & $.60 \mathrm{NS}$ & & & & $\mathbf{F}$ & & & & \\
\hline & .15 & .13 & $\begin{array}{l}.14 \\
.15\end{array}$ & .145 & Neg. & & & $\mathrm{GSC} / \mathrm{k}$ & & & & 02 & $<00$ & O & $1.00 \mathrm{NS}$ \\
\hline UInd/ICPS & .25 & .26 & .26 & .245 & Neg. & .022 & $.72 \mathrm{NS}$ & $\mathrm{GSC} / \mathrm{K}$ & $\begin{array}{l}.03 \\
.02 \\
\end{array}$ & .02 & $\begin{array}{l}.02 \\
.02 \\
\end{array}$ & .02 & $<.00$ & .004 & $1.00 \mathrm{NS}$ \\
\hline & .23 & .26 & .21 & & & & & & & & $\mathrm{~s}$ & & & & \\
\hline Exxon/DCPAS & $\cdot 14,1$ & .138 & - & .134 & Ne8. & .005 & $.007 \mathrm{NS}$ & & & & & & & & \\
\hline & .132 & .133 & - & & & & & $\operatorname{Gsc} / \mathrm{A}$ & .00 & .00 & .00 & .00 & - & - & - \\
\hline & .129 & .132 & - & & & & & & .00 & .00 & .00 & & & & \\
\hline GRPG/MWPS & .23 & .21 & - & .218 & Neg. & .01 & $.20 \mathrm{NS}$ & & & & & & & & \\
\hline & .21 & .22 & - & & & & & & & & & & & & \\
\hline
\end{tabular}


Table 1. Analytical data for USGS-W-2 (cont.)

\begin{tabular}{|c|c|c|c|c|c|c|c|c|c|c|c|c|c|c|c|}
\hline \multirow[b]{4}{*}{ Org./Method. } & \multirow[b]{3}{*}{ Bottle } & & & \multirow[b]{4}{*}{ Mean } & & & \multirow{4}{*}{$\begin{array}{r}F \\
\text { ratio } \\
\end{array}$} & \multirow{4}{*}{ Org./Meth. } & \multirow{2}{*}{$\frac{\text { Boctle }}{1}$} & \multicolumn{2}{|c|}{ Number } & & Bott le & Error & $\boldsymbol{F}$ \\
\hline & & & & & Standard De & eviation & & & & 2 & 3 & Mean & $\underline{2 d f}$ & $3 \mathrm{df}$ & ratio \\
\hline & & & geer & & Bott1e & Error & & & & & & & & & \\
\hline & 1 & 2 & 3 & & $2 \mathrm{df}$ & $3 \mathrm{df}$ & & & & & $\mathrm{Ag}_{\mathrm{g}}$ & & & & \\
\hline & & & LI & & & & & USGSR/AAS & $0 . \alpha_{4} 2$ & 0.046 & 0.058 & 0.048 & - & - & - \\
\hline UInd/ & 1.08 & - & - & - & - & - & - & & - & .040 & .056 & & & & \\
\hline CRPG & .10 & .09 & - & .098 & .01 & .005 & $9.00 \mathrm{NS}$ & & & & As & & & & \\
\hline & & & $\mathrm{Fe}_{2} \mathrm{O}_{3} \mathrm{~T}$ & & & & & $\mathrm{Gsc} / \mathrm{c}$ & .6 & .7 & .7 & .7 & Neg. & 0.06 & $0.50 \mathrm{NS}$ \\
\hline *BMNH/Color & 10.57 & 10.83 & 10.58 & 10.77 & - & - & - & & .7 & .6 & .7 & & & & \\
\hline & 10.86 & 10.89 & 10.87 & & & & & $\begin{array}{l}\text { Tor on/INAA } \\
\text { HMI/SSMS }\end{array}$ & $\overline{1.2}$ & 1.9 & - & $\begin{array}{l}<2 \\
1.55\end{array}$ & Neg. & .63 &. \\
\hline *BMNH/XRF & 10.84 & 10.97 & 10.90 & 10.92 & Neg. & .058 & $0.31 \mathrm{Ns}$ & & 2.6 & 1.0 & - & & & & \\
\hline & 10.96 & 10.92 & 10.96 & & & & 1.hONS & & 1.4 & 1.2 & - & & & & \\
\hline *BMNH/ XRF & $\begin{array}{l}10.94 \\
10.84\end{array}$ & $\begin{array}{l}10.48 \\
10.81\end{array}$ & $\begin{array}{l}10.82 \\
10.73\end{array}$ & 10.77 & .067 & .145 & $1.42 \mathrm{NS}$ & GCL/Spph & 1.8 & .6 & .5 & 1.28 & .47 & .56 & 2.4 ONS \\
\hline $\mathrm{GSC} / \mathrm{A}$ & 10.8 & 10.9 & 10.9 & 10.8 & .05 & .10 & $1.50 \mathrm{Ns}$ & KJell/INAA & $\begin{array}{l}2.1 \\
1.4\end{array}$ & $\begin{array}{r}.9 \\
1.4\end{array}$ & $\begin{array}{l}1.8 \\
1.5\end{array}$ & 1.4 & - & - & - \\
\hline $\mathrm{GSC} / \mathrm{F}^{*}$ & $\begin{array}{l}10.6 \\
10.75\end{array}$ & $\begin{array}{l}10.8 \\
10.81\end{array}$ & $\begin{array}{l}10.8 \\
10.77\end{array}$ & 10.78 & Neg. & .04 & $.10 \mathrm{NS}$ & & & & B & & & & \\
\hline & 10.80 & 10.72 & 10.80 & & & & & $\mathrm{GCL} / \mathrm{Spph}$ & 10.1 & $9 \cdot 4$ & 11.9 & 10.7 & Neg. & 1.11 & $.4 \mathrm{NSS}$ \\
\hline BIO/AAS & 10.66 & 10.61 & 10.69 & 10.60 & .01 & .09 & $1.04 \mathrm{NS}$ & & 11.7 & 10.9 & 10.3 & & & & \\
\hline & 10.64 & 10.44 & 10.55 & & & & & & & & $\mathbf{B a}$ & & & & \\
\hline *NIM/XRF & 11.0 & $10 \cdot 9$ & 10.8 & 10.82 & Neg. & .10 & $.33 \mathrm{NS}$ & $\mathrm{GSC} / \mathrm{N}$ & 185 & 205 & 190 & 188 & Neg. & 10 & $.50 \mathrm{NS}$ \\
\hline & 10.7 & 10.9 & 10.8 & & & & & & 180 & 180 & 190 & & & & \\
\hline & 10.7 & 10.8 & 10.7 & & & & & WHOI/XRF & 178.5 & 182.7 & 187.6 & 183.0 & 5.1 & .7 & 117 \\
\hline & 10.8 & 10.8 & 10.9 & & & & & & 177.5 & 182.7 & 188.9 & & & & \\
\hline $\mathrm{NIM} / \mathrm{Dichr}$ & 10.94 & 10.80 & 11.19 & 10.97 & .13 & .07 & $8.67 \mathrm{NS}$ & UWUrz/XRF & 170 & 174 & 170 & 172.3 & Neg. & $3 \cdot 3$ & $.02 \mathrm{NS}$ \\
\hline & 10.95 & 10.90 & 11.06 & & & & & & 175 & 170 & 175 & & & & \\
\hline Parma/Color & 10.94 & 11.00 & - & 10.90 & .060 & .060 & $3 \cdot 91 \mathrm{NS}$ & B i rm/XRF & 168.4 & 169.6 & - & 162.7 & Neg. & 10.02 & $.28 \mathrm{NS}$ \\
\hline & 10.80 & 10.94 & - & & & & & & 164.9 & 170.8 & - & & & & \\
\hline & 10.83 & 10.92 & & & & & & & $L_{4} 8.3$ & 154.2 & - & & & & \\
\hline WHOI/XRF & 10.98 & 10.95 & 10.97 & 10.94 & Neg. & .047 & $.23 \mathrm{NS}$ & Nott/XRF & 229.39 & 231.31 & - & 233.46 & 3.68 & 5.36 & $2.41 \mathrm{NS}$ \\
\hline & 10.88 & 10.90 & 10.94 & & & & & & 239.55 & $225 \cdot 77$ & - & & & & \\
\hline UWurz/XRF & 10.95 & 10.95 & 10.90 & 10.90 & Neg. & .06 & $.90 \mathrm{NS}$ & & 241.64 & 233.09 & - & & & & \\
\hline & 10.95 & 10.80 & 10.85 & & & & & *Toron/INAA & 350 & 230 & 240 & 286.7 & Neg. & 48 & $\cdot 72 \mathrm{NS}$ \\
\hline $\mathrm{WSU} / \mathrm{XRF}$ & 10.94 & 11.26 & 11.01 & 11.04 & .19 & .06 & 23.40 & & 290 & 320 & 290 & & & & \\
\hline & 10.81 & 11.24 & 10.97 & & & & & & 290 & $<200$ & 330 & & & & \\
\hline USGSR/XRF & 10.93 & 10.78 & 10.93 & 10.85 & .09 & .04 & 10.38 & *HMI/INAA-W & 180 & 200 & - & 172 & Neg。 & 21.6 & $.0_{4} \mathrm{NS}$ \\
\hline *Tor on/INAA & $\begin{array}{r}10.86 \\
7.79\end{array}$ & $\begin{array}{r}10.70 \\
7.63\end{array}$ & 10.92 & 7.59 & Neg. & .23 & $.38 \mathrm{NS}$ & & 180 & 150 & - & & & & \\
\hline & 7.46 & 7.74 & 7.80 & & & & & & 150 & 170 & - & & & & \\
\hline & 7.65 & 7.13 & 7.68 & & & & & *HM I/INAA-B & 230 & 270 & - & 237 & Neg. & 33.7 & $.53 \mathrm{NS}$ \\
\hline *HMI/INAA-W & 7.69 & 8.12 & - & $7 \cdot 81$ & Neg. & .27 & $<.00 \mathrm{NS}$ & & 200 & 270 & - & & & & \\
\hline & $7 \cdot 91$ & 7.41 & - & & & & & NERF/INAA & 250 & 200 & - & & & & \\
\hline & 7.83 & 7.89 & - & & & & & NERF/INAA & - & 180 & - & 150 & - & - & - \\
\hline *HMI/INAA-B & 7.60 & $7 \cdot 96$ & - & 7.65 & Neg. & .22 & $<.00 \mathrm{NS}$ & LASL/INAA-1 & 135 & - & - & & Neg & 91 & 20NS \\
\hline & $7 \cdot 71$ & $7 \cdot 36$ & - & & & & & (大) & $\begin{array}{l}200 \\
210\end{array}$ & $\begin{array}{l}400 \\
170\end{array}$ & - & 247 & Neg. & $7 \pm$ & 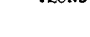 \\
\hline & 7.65 & 7.63 & - & & & & & & 280 & 220 & - & & & & \\
\hline *NERF/INAA & 7.82 & 7.91 & $7 \cdot 74$ & $7 \cdot 91$ & .14 & .20 & $1.97 \mathrm{NS}$ & Open/INAA & 161 & 182 & - & 177 & Neg. & 19 & $<.00 \mathrm{NS}$ \\
\hline & 8.24 & 8.15 & 7.62 & & & & & & 202 & 161 & - & & & & \\
\hline *LASL/INAA-1 & 7.47 & 7.24 & - & 7.28 & .083 & .139 & $2.07 \mathrm{NS}$ & & 165 & 189 & - & & & & \\
\hline & 7.46 & 7.11 & - & & & & & USGSR/INAA & 180 & 200 & 166 & 174.3 & 11.2 & 11.8 & $2.82 \mathrm{NS}$ \\
\hline & 7.15 & $7 \cdot 24$ & - & & & & & & 160 & 180 & 160 & & & & \\
\hline Open/INAA & 10.58 & 11.06 & - & 10.55 & Neg. & .40 & $.69 \mathrm{NS}$ & UInd/rCPS & 171 & 169 & 170 & 168.2 & Neg. & 2.7 & $.29 \mathrm{NS}$ \\
\hline & 10.82 & 10.10 & - & & & & & & 166 & 165 & 168 & & & & \\
\hline & 10.67 & 10.09 & - & & & & & CRPG/MWPS & 185 & 184 & - & 184.5 & 1.3 & $\cdot 7$ & $8.00 \mathrm{NS}$ \\
\hline *USGSR/INAA & $7 \cdot 70$ & $7 \cdot 74$ & $7 \cdot 32$ & $7 \cdot 55$ & $\cdot u_{4}$ & .14 & $2.99 \mathrm{NS}$ & & 186 & 183 & - & & & & \\
\hline & $7 \cdot 39$ & 7.69 & 7.44 & & & & & USGSR/ORS & $1_{4} 0$ & 170 & 160 & 160 & 8.2 & 8.2 & $3.00 \mathrm{NS}$ \\
\hline LASL/INAA & 10.55 & 10.74 & - & 10.83 & Neg. & .25 & $.24 \mathrm{NS}$ & & 160 & 170 & 160 & & & & \\
\hline & 10.92 & 11.21 & - & & & & & HMI/SSMS & 131.6 & 82.6 & - & 89.6 & 19.2 & 20.0 & $3.78 \mathrm{NS}$ \\
\hline & 10.86 & 10.68 & - & & & & & & 78.4 & 74.9 & - & & & & \\
\hline LASL/XRF & 10.68 & 10.76 & - & 10.72 & - & - & - & & 106.4 & 63.7 & - & & & & \\
\hline Tohok/IPAA & $11.0_{4}$ & 10.91 & 11.09 & 10.95 & .08 & .13 & $1.68 \mathrm{NS}$ & ETH $/$ XRF-L $\alpha_{2}$ & 171 & 171 & 175 & 172 & - & - & - \\
\hline & 10.72 & 10.84 & 11.09 & & & & & $\mathrm{ETH} / \mathrm{XRF-L} B$ & 179 & 179 & 181 & 180 & - & - & - \\
\hline UInd/ICPS & 11.3 & 10.8 & 10.9 & 10.98 & .32 & .07 & 41.33 & Kjell/ICPS & 169 & 172 & 168 & 170 & - & - & - \\
\hline & 11.4 & 10.7 & 10.8 & & & & & /INAA & 180 & 170 & 140 & 163 & - & - & - \\
\hline Exxon/DCPAS & $\begin{array}{l}10.87 \\
10.85\end{array}$ & $\begin{array}{l}10.86 \\
10.77\end{array}$ & - & 10.80 & .05 & .035 & $7 \cdot 14 \mathrm{NS}$ & & & & $\mathrm{Be}$ & & & & \\
\hline & 10.78 & 10.74 & - & & & & & B IO/AAS & 1.2 & 1.1 & 1.3 & 1.2 & .06 & .09 & 1.8ONS \\
\hline CRPG/MWPS & 10.77 & 10.69 & - & 10.74 & Neg. & .10 & $.40 \mathrm{NS}$ & & 1.0 & 1.1 & 1.2 & & & & \\
\hline & 10.64 & 10.85 & - & & & & & GCL/AAS & 1.0 & 1.2 & 1.2 & 1.13 & Neg. & .12 & $.50 \mathrm{NS}$ \\
\hline$* \mathrm{Kje} 11 / \mathrm{AAS}$ & $7 \cdot 25$ & 7.25 & 7.40 & $7 \cdot 30$ & - & - & - & & 1.2 & 1.0 & 1.2 & & & & \\
\hline /ICPS & 7.41 & 7.43 & $7 \cdot 36$ & 7.40 & - & - & - & & & & & & & & \\
\hline$/ \mathrm{INAA}$ & $7 \cdot 10$ & 7.57 & 7.02 & 7.23 & - & - & - & & & & & & & & \\
\hline /XRF & 7.48 & 7.41 & 7.55 & 7.49 & - & '- & - & & & & & & & & \\
\hline
\end{tabular}


Table 1. Analytical daca for USGS-W-2 (cont.)

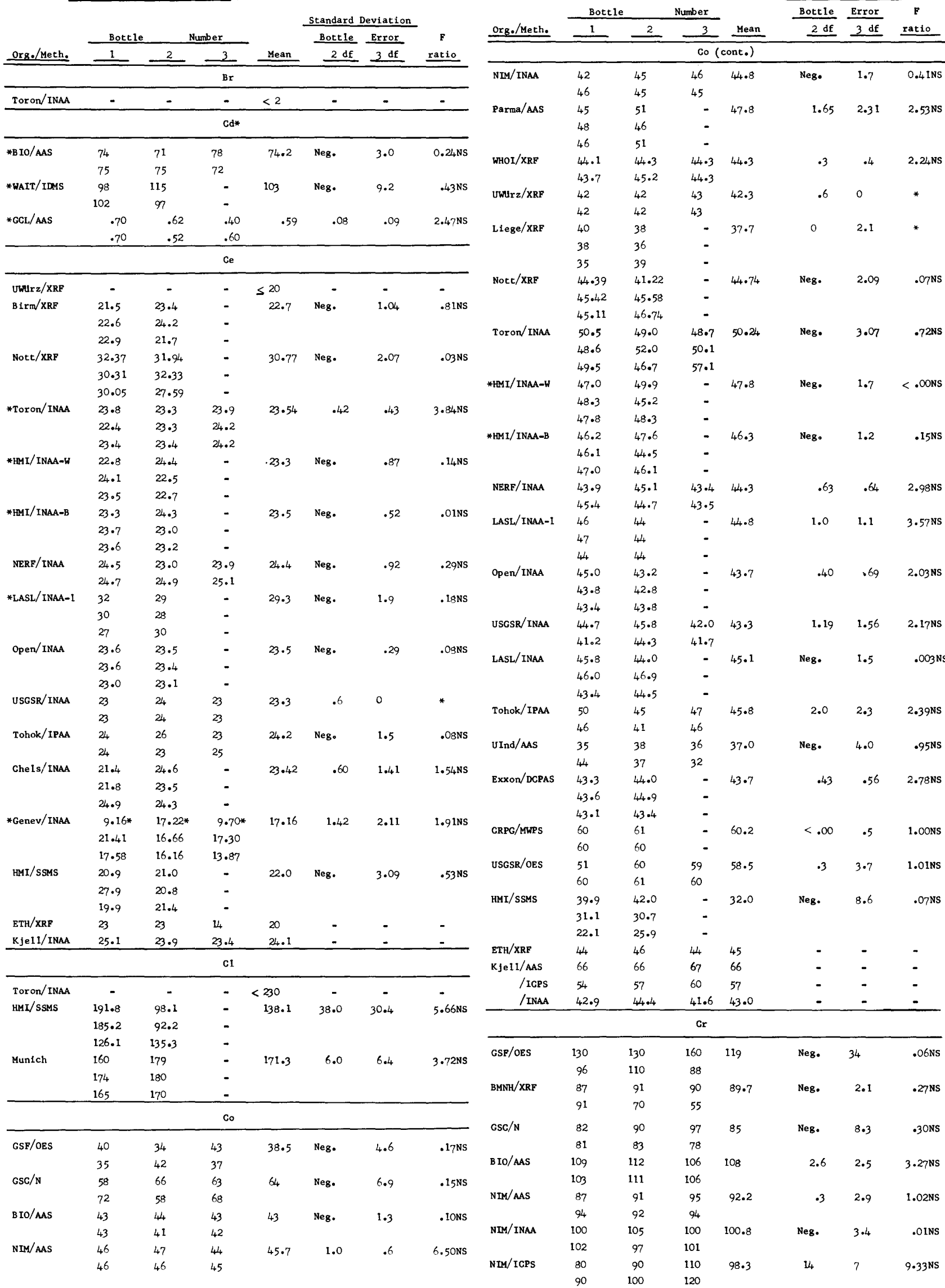


Table 1. Analytical data for USGS-W-2 (cont.)

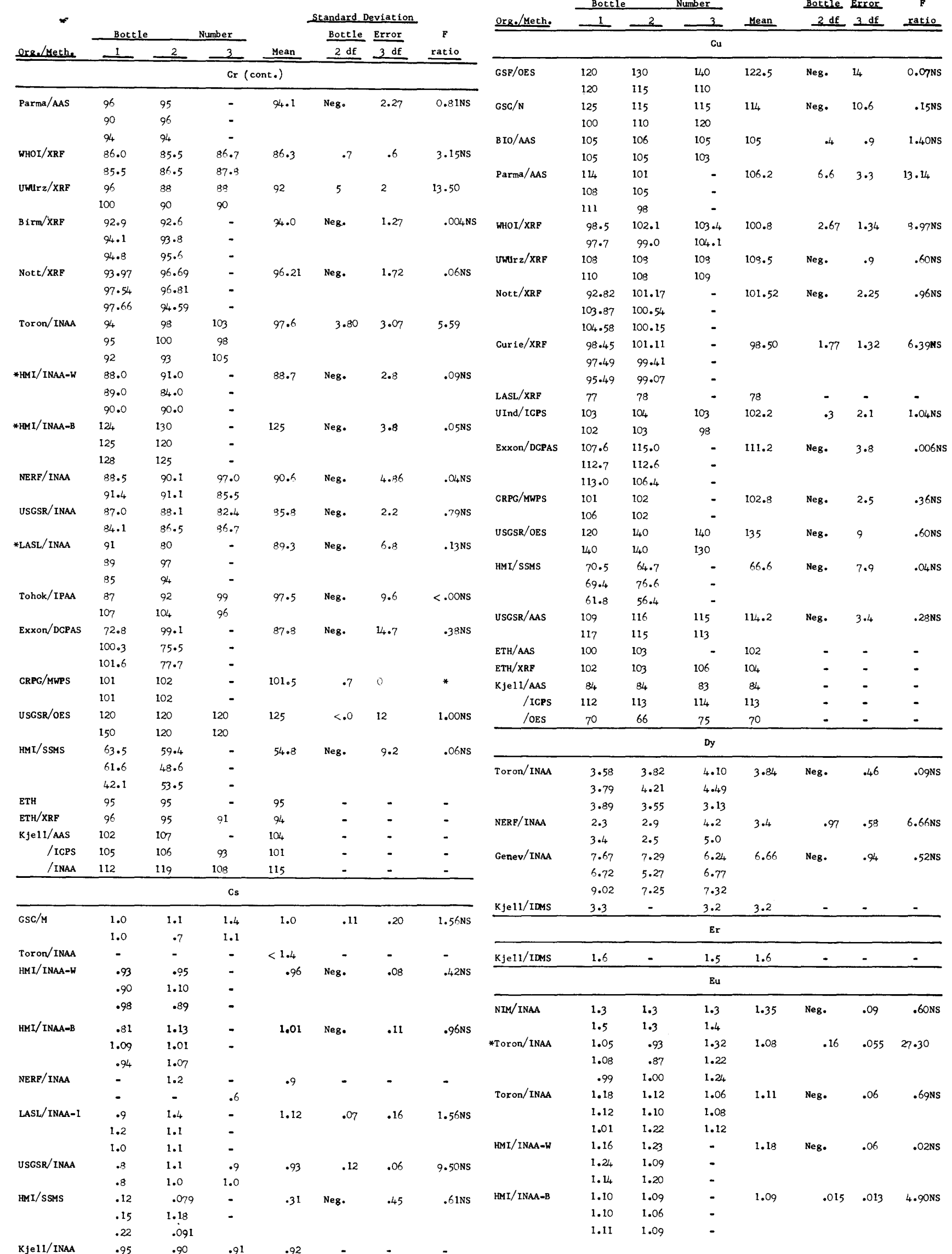


Table 1. Analytical data for USGS-W-2 (cont.)

\begin{tabular}{|c|c|c|c|c|c|c|c|}
\hline Orge/Metho & Bottle & \multicolumn{2}{|r|}{ Number } & Mean & $\begin{array}{l}\text { Standard } \\
\text { Bottle } \\
2 \mathrm{df} \\
\end{array}$ & $\begin{array}{l}\text { eviation } \\
\text { Errox } \\
3 \mathrm{df} \\
\end{array}$ & $\begin{array}{c}\mathbf{P} \\
\text { ratio } \\
\end{array}$ \\
\hline \multicolumn{8}{|c|}{ Eu (cont.) } \\
\hline \multirow[t]{2}{*}{ NERF/INAA } & 1.22 & 1.07 & 1.21 & 1.16 & 0.054 & 0.046 & $3.75 \mathrm{NS}$ \\
\hline & 1.22 & 1.12 & 1.11 & & & & \\
\hline \multirow[t]{3}{*}{ LASL/INAA-I } & 66 & 1.09 & - & 1.06 & .02 & .06 & $1.55 \mathrm{NS}$ \\
\hline & 1.12 & 1.09 & - & & & & \\
\hline & 1.00 & 1.08 & - & & & & \\
\hline \multirow[t]{3}{*}{ Open/INAA } & 1.18 & 1.15 & - & 1.16 & .02 & .02 & $4.84 \mathrm{NS}$ \\
\hline & 1.16 & 1.16 & - & & & & \\
\hline & 1.20 & 1.12 & - & & & & \\
\hline \multirow[t]{2}{*}{ USGSR/INAA } & 1.07 & 1.18 & 1.06 & 1.04 & .04 & .07 & $1.79 \mathrm{NS}$ \\
\hline & .98 & 1.05 & .95 & & & & \\
\hline \multirow[t]{3}{*}{ LASL/INAA } & 1.20 & 1.22 & - & 1.24 & Neg. & .07 & $.94 \mathrm{NS}$ \\
\hline & 1.23 & 1.24 & - & & & & \\
\hline & 1.37 & 1.19 & - & & & & \\
\hline Chels/INAA & 1.06 & 1.17 & - & 1.08 & .03 & .05 & $2.59 \mathrm{NS}$ \\
\hline & 1.02 & 1.05 & - & & & & \\
\hline & 1.06 & 1.10 & - & & & & \\
\hline Genev/INAA & 1.78 & 1.37 & 1.60 & 1.60 & Neg. &.$u$ & $.88 \mathrm{NS}$ \\
\hline & 1.60 & 1.65 & 1.63 & & & & \\
\hline & 1.93 & 1.70 & 1.53 & & & & \\
\hline HMI/SSMS & .53 & .68 & - & 1.06 & Neg. & .43 & $.45 \mathrm{NS}$ \\
\hline & 1.50 & 1.01 & - & & & & \\
\hline & 1.49 & 1.13 & - & & & & \\
\hline Kjell/IDMS & .9 & - & .8 & .85 & - & - & - \\
\hline /INAA & 1.11 & 1.02 & 1.08 & 1.07 & - & - & - \\
\hline & & & $\mathbf{F}$ & & & & \\
\hline HMI/SSMS & 103 & 126 & - & $164 \cdot 3$ & Neg. & 53.4 & $.54 \mathrm{NS}$ \\
\hline & 248 & 159 & - & & & & \\
\hline & 190 & 160 & - & & & & \\
\hline Munich & 210 & 200 & - & 198.3 & 2.9 & 6.4 & $1.60 \mathrm{NS}$ \\
\hline & 200 & 195 & - & & & & \\
\hline & 195 & 190 & - & & & & \\
\hline & & & $\mathrm{Ga}$ & & & & \\
\hline GSF/OES & 26 & 25 & 32 & 21.7 & Neg. & 8.7 & $.11 \mathrm{NS}$ \\
\hline & 15 & 16 & 16 & & & & \\
\hline UWUrz/XRF & 17 & 17 & 16 & 16 & 0 & 1.2 & * \\
\hline & 15 & 15 & 16 & & & & \\
\hline $\mathrm{BIrm} / \mathrm{XRF}$ & 17.5 & 16.9 & - & 16.2 & Neg. & 1.3 & $.001 \mathrm{NS}$ \\
\hline & 14.3 & 16.5 & - & & & & \\
\hline & 16.6 & 15.1 & - & & & & \\
\hline Nott/XRF & 16.62 & 16.77 & - & 16.87 & .35 & .52 & $2.33 \mathrm{NS}$ \\
\hline & 15.89 & 17.55 & - & & & & \\
\hline & 17.13 & 17.27 & - & & & & \\
\hline Curie/XRF & 17.29 & 18.53 & - & 18.03 & .24 & .69 & $1.35 \mathrm{NS}$ \\
\hline & 17.07 & 18.57 & - & & & & \\
\hline & 18.75 & 17.97 & - & & & & \\
\hline USGSR/OES & 20 & 21 & 21 & 20.8 & $\cdot 3$ & $\cdot 7$ & $1.33 \mathrm{NS}$ \\
\hline & 21 & 20 & 22 & & & & \\
\hline HMI/SSMS & $7 \cdot 9$ & 9.6 & - & 8.32 & Neg. & 1.1 & $.03 \mathrm{NS}$ \\
\hline & 9.4 & 8.2 & - & & & & \\
\hline & 7.9 & 6.9 & - & & & & \\
\hline $\mathrm{ETH} / \mathrm{XRF}$ & 20 & 20 & 19 & 20 & - & - & - \\
\hline & & & Gd & & & & \\
\hline Open/INAA & 4.3 & 4.0 & - & 4.38 & .45 & .52 & $3.31 \mathrm{NS}$ \\
\hline & 3.9 & $5 \cdot 3$ & - & & & & \\
\hline & 3.8 & 5.0 & - & & & & \\
\hline USGSR/INAA & 3.6 & 4.1 & 3.2 & 3.4 & .16 & .40 & $1.30 \mathrm{NS}$ \\
\hline & 3.1 & $3 \cdot 3$ & 2.9 & & & & \\
\hline Kjel1/IDMs & - & - & 2.8 & - & - & - & - \\
\hline & & & $\mathrm{Hf}$ & & & & \\
\hline NIM/INAA & 2.4 & 2.9 & 3.0 & 2.73 & Neg. & .29 & $.14 \mathrm{NS}$ \\
\hline & 2.9 & 2.6 & 2.6 & & & & \\
\hline Toron/INAA & 3.0 & 2.4 & 2.4 & 2.62 & Neg. & .27 & $.76 \mathrm{NS}$ \\
\hline & 2.6 & 2.6 & 2.5 & & & & \\
\hline & 2.5 & 2.4 & 3.1 & & & & \\
\hline
\end{tabular}

\begin{tabular}{|c|c|c|c|c|c|c|c|}
\hline \multirow{3}{*}{ Org./Meth. } & \multirow[b]{2}{*}{ Bottle } & \multirow{2}{*}{\multicolumn{2}{|c|}{ Number }} & \multicolumn{4}{|c|}{ Standard Deviation } \\
\hline & & & & \multirow[b]{2}{*}{ Mean } & \multirow{2}{*}{$\begin{array}{r}\text { Botile } \\
2 d f \\
\end{array}$} & \multirow{2}{*}{ Exrox } & \multirow{2}{*}{$\begin{array}{c}F \\
\text { ratio } \\
\end{array}$} \\
\hline & 1 & 2 & 3 & & & & \\
\hline \multicolumn{8}{|c|}{ Hf (cont.) } \\
\hline \multirow[t]{3}{*}{ HMI/INAA-W } & 2.72 & 2.88 & - & 2.73 & Neg. & 0.11 & $0.11 \mathrm{NS}$ \\
\hline & 2.78 & 2.58 & - & & & & \\
\hline & 2.70 & 2.67 & - & & & & \\
\hline \multirow[t]{3}{*}{ HAMI/INAA-B } & 2.51 & 2.48 & - & 2.45 & Neg. & .046 & .5 INS \\
\hline & 2.41 & 2.40 & - & & & & \\
\hline & 2.47 & 2.43 & - & & & & \\
\hline \multirow[t]{2}{*}{ NERF/ INAA } & 2.66 & 2.77 & 2.43 & 2.54 & Neg. & .19 & $.35 \mathrm{NS}$ \\
\hline & 2.57 & 2.31 & 2.48 & & & & \\
\hline \multirow[t]{3}{*}{ LASL/INAA-I } & 2.8 & 2.7 & - & 2.72 & Neg. & .15 & $.08 \mathrm{NS}$ \\
\hline & 2.5 & 2.7 & - & & & & \\
\hline & 2.9 & 2.7 & - & & & & \\
\hline \multirow[t]{3}{*}{ Open/INAA } & 2.50 & 2.49 & - & 2.35 & Neg. & .20 & $.04 \mathrm{NS}$ \\
\hline & 2.48 & 2.31 & - & & & & \\
\hline & 2.03 & 2.31 & - & & & & \\
\hline \multirow[t]{2}{*}{ USGSR/INAA } & 2.7 & 2.7 & 2.4 & 2.57 & .15 & .13 & $3.70 \mathrm{Ns}$ \\
\hline & 2.4 & 2.8 & 2.4 & & & & \\
\hline \multirow[t]{3}{*}{ LASL/INAA } & 2.7 & 2.8 & - & 2.7 & - & - & - \\
\hline & 2.3 & 3.3 & - & & & & \\
\hline & - & 2.4 & - & & & & \\
\hline \multirow[t]{2}{*}{$\mathrm{Kjel1} / \mathrm{INAA}$} & 2.0 & 2.6 & 2.7 & 2.4 & - & - & - \\
\hline & & & но & & & & \\
\hline \multirow[t]{2}{*}{ USGSR/INAA } & .8 & .8 & .4 & .62 & Neg. & .17 & $.76 \mathrm{NS}$ \\
\hline & .5 & .6 & .6 & & & & \\
\hline \multirow[t]{3}{*}{ Che Is/INAA } & .71 & .64 & - & .71 & Neg: & .08 & $.32 \mathrm{NS}$ \\
\hline & .66 & .66 & - & & & & \\
\hline & .82 & .78 & - & & & & \\
\hline \multirow[t]{3}{*}{ Genev/INAA } & 1.36 & 1.33 & 1.28 & 1.26 & Neg. & .32 & $.02 \mathrm{NS}$ \\
\hline & 1.24 & 1.12 & 1.25 & & & & \\
\hline & 1.02 & 1.08 & 1.31 & & & & \\
\hline
\end{tabular}

\begin{tabular}{llllllll}
\hline \multicolumn{7}{c}{ La } \\
\hline NIM/INAA & 13.1 & 13.4 & 14.2 & 13.6 & .61 & .09 & 90.6 \\
& 13.1 & 13.2 & 14.3 & & & &
\end{tabular}

$\begin{array}{llll}13.1 & 13.2 \quad 14.3\end{array}$

$\begin{array}{llllllll}\text { UWH z } / \text { XRF } & 11 & 9 & 12 & 10.7 & 1.5 & 0\end{array}$

$\begin{array}{lllllllll}\mathrm{Birm} / \mathrm{XRF} & 9.7 & 9.6 & - & 9.6 & .20 & .40 & 1.80 \mathrm{NS}\end{array}$

$\begin{array}{lcllllll} & 9.6 & 10.1 & - & & & & \\ \text { Nott } / \mathrm{XRF} & 10.91 & 12.85 & - & 12.50 & .62 & .68 & 3.55 \mathrm{NS}\end{array}$

$12.39-13.24$

$12.64 \quad 12.97$

Toron/INA4 8.66

$\begin{array}{llll}10.4 & 9.90 \quad .62 & .47 & 6.13\end{array}$

$9.50 \quad 9.94 \quad 10.1$

$9.59 \quad 9.69 \quad 11.3$

HMI/INAA-W $\quad 9.98 \quad 10.2$

HMI/INAA-B $\quad 11.2 \quad 11.2$

$10.7 \quad 10.6$

$10.7 \quad 11$.

NERF/INAA

$\begin{array}{rr}9.94 & 10.32 \\ 10.22 & 10.04\end{array}$

Open/INAA $\quad 11.1 \quad 10.8$

$12.6 \quad 10.3$

$9.9 \quad 10.9$

USGSR/INAA

11

LASL/INAA

9.1

9.13

9.29

Chels/INAA

10.6

10.6

11.3

Genev/INAA

11.3

$\begin{array}{lll}10.98 & 10.76\end{array}$

Exxon/DCPAS $18.2 \quad 17.2 \quad$ -

$16.9 \quad 19.6$ -

$18.9 \quad 18.6$ 
Table 1. Analytical data for USGS-W-2 (cont.)

\begin{tabular}{|c|c|c|c|c|c|c|c|}
\hline \multirow[b]{2}{*}{ Org./Meth. } & \multicolumn{2}{|c|}{ Bottile } & \multirow{2}{*}{$\begin{array}{r}\text { Number } \\
3 \\
\end{array}$} & \multirow[b]{2}{*}{ Mean } & \multicolumn{2}{|c|}{ Standard Deviation } & \multirow{2}{*}{$\begin{array}{r}F \\
\text { ratio } \\
\end{array}$} \\
\hline & 1 & 2 & & & $\underline{2 \mathrm{df}}$ & $3 \mathrm{df}$ & \\
\hline \multicolumn{8}{|c|}{ La (cont.) } \\
\hline \multirow[t]{3}{*}{ HMAI/SSHS } & 10.5 & $9 \cdot 7$ & - & 12.1 & 1.2 & 1.8 & $2.39 \mathrm{NS}$ \\
\hline & 4.6 & 11.2 & - & & & & \\
\hline & 14.5 & 11.8 & - & & & & \\
\hline \multirow[t]{2}{*}{ Kje11/INAA } & 12.3 & 10.0 & 9.8 & 10.7 & $=$ & $=$ & - \\
\hline & & & Li & & & & \\
\hline \multirow[t]{2}{*}{$\mathrm{GSC} / \mathrm{M}$} & 11 & 13 & 10 & 10.2 & Neg. & 3.4 & - IgNS \\
\hline & 10 & 5 & 12 & & & & \\
\hline \multirow[t]{2}{*}{ B IO/AAS } & 10.0 & $9 \cdot 7$ & 9.8 & 9.8 & .1 & .1 & $2.08 \mathrm{NS}$ \\
\hline & 10.0 & 10.0 & 9.6 & & & & \\
\hline \multirow[t]{3}{*}{ Parma/AAS } & 8.3 & 9.6 & - & 9.18 & .93 & $.4 ?$ & 12.64 \\
\hline & 9.2 & 10 & - & & & & \\
\hline & 8.0 & 10 & - & & & & \\
\hline \multirow[t]{2}{*}{ UWUIrz/XRF } & 9 & 11 & 9 & 9.5 & .8 & .4 & $9.00 \mathrm{NS}$ \\
\hline & 9 & 10 & 9 & & & & \\
\hline \multirow[t]{2}{*}{ USGSR/OES } & 6 & 7 & 8 & 6.8 & Neg. & 1.2 & $.78 \mathrm{NS}$ \\
\hline & 8 & 5 & 7 & & & & \\
\hline \multirow[t]{2}{*}{ USGSD/AAS } & 8 & 8 & 8 & 8 & - & - & - \\
\hline & 8 & $s$ & 8 & & & & \\
\hline \multirow[t]{2}{*}{ USGSR/AAS } & 9.6 & 9.6 & 9.8 & 9.78 & Neg. & .18 & $.21 \mathrm{NS}$ \\
\hline & 9.9 & 9.9 & 9.9 & & & & \\
\hline
\end{tabular}

\begin{tabular}{|c|c|c|c|c|c|c|c|}
\hline & & & Lu & & & & \\
\hline \multirow[t]{2}{*}{ NIM/INAA } & .37 & $\cdot 34$ & $\cdot 37$ & .37 & .008 & .017 & $1.47 \mathrm{NS}$ \\
\hline & .39 & $\cdot 37$ & .39 & & & & \\
\hline \multirow[t]{3}{*}{ Toron/INAA } & .25 & .31 & .26 & .282 & $\mathrm{Ne}_{8}$. & .037 & $.35 \mathrm{NS}$ \\
\hline & .25 & .33 & .29 & & & & \\
\hline & .33 & .25 & .27 & & & & \\
\hline \multirow[t]{3}{*}{ HMI/INAA-W } & .381 & .385 & - & .372 & Neg. & .012 & .0 NS \\
\hline & .358 & .360 & - & & & & \\
\hline & .373 & .376 & - & & & & \\
\hline \multirow[t]{2}{*}{$\mathrm{HMI} / \mathrm{INAA-B}$} & .330 & .359 & - & .342 & Neg. & .017 & $.08 \mathrm{NS}$ \\
\hline & .355 & .321 & - & & & & \\
\hline
\end{tabular}

$\begin{array}{llllllll} & .355 & .321 & - & & & & \\ \text { NERF/INAA } & .335 & .352 & - & & & & \\ & .37 & .44 & .55 & .52 & \text { Neg. } & .27 & .64 \text { NS }\end{array}$

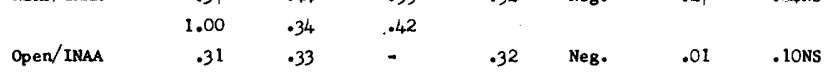

$\begin{array}{lllllllll} & .34 & .31 & - & & & & & \\ & .32 & .32 & - & & & & \\ \text { USGSR/INAA } & .33 & .33 & .32 & .333 & .016 & .025 & 1.76 \mathrm{NS}\end{array}$

$\begin{array}{llllllll}\text { USGSR/INAA } & .33 & .33 & .32 & .333 & .016 & .025 & 1.76 \mathrm{NS}\end{array}$

$\begin{array}{lllllllll}\text { Chels/INAA } & .31 & .32 & - & .34 & \text { Neg. } & .04 & .47 \mathrm{NS}\end{array}$

$\begin{array}{lllllllll} & .31 & .36 & - & & & & & \\ & .38 & .38 & - & & & & \\ \text { Genev/INAA } & .66 & .60 & .71 & .63 & .05 & .04 & 3.73 \mathrm{NS}\end{array}$

\begin{tabular}{llllllll} 
& .66 & .60 & .71 & .63 & .05 & .04 & $3.73 N 5$ \\
& .63 & .54 & .64 & & & & \\
$\mathrm{Kje11} / \mathrm{INAA}$ & .73 & .54 & .60 & & & \\
\hline & .31 & .31 & .30 & .31 & - & - & - \\
$\mathrm{Mn}$ & &
\end{tabular}

\begin{tabular}{lrrrrrrr}
\hline BIO/AAS & 1255 & 1250 & 1265 & 1256 & 7 & 8 & 2.53 NS \\
& 1270 & 1240 & 1255 & & & & \\
NERE/INAA & 1455 & 1500 & 1580 & 1540 & 46 & 40 & $3.53 N S$ \\
& 1510 & 1580 & 1600 & & & & \\
LASL/INAA-1 & 1290 & 1350 & - & 1333 & 0 & .29 & $*$ \\
& 1360 & 1330 & - & & & & \\
& 1350 & 1320 & - & & & & \\
LASL/XRF & 1234 & 1245 & - & 1240 & - & - & - \\
USGSR/OES & 1000 & 900 & 950 & 1060 & 112 & 231 & $1.47 \mathrm{NS}$ \\
& 1500 & 810 & 1200 & & & & \\
HMI/SSMS & 1233 & 1119 & - & 1133 & Neg. & 242 & $.14 \mathrm{NS}$ \\
& 1250 & 1423 & - & & & &
\end{tabular}

\begin{tabular}{|c|c|c|c|c|c|c|c|}
\hline & 803 & 968 & - & & & & \\
\hline & & & Mo & & & & \\
\hline \multirow[t]{2}{*}{ UInd/ICPS } & .73 & 1.2 & 1.0 & 1.06 & Neg. & .20 & $.43 \mathrm{NS}$ \\
\hline & 1.2 & 1.1 & 1.1 & & & & \\
\hline \multirow[t]{2}{*}{ USGSR/Spph } & .37 & .37 & .26 & .36 & Neg. & .06 & $\cdot 38 \mathrm{NS}$ \\
\hline & .38 & .37 & .40 & & & & \\
\hline
\end{tabular}

\begin{tabular}{|c|c|c|c|c|c|c|c|}
\hline \multirow[b]{3}{*}{ Orgo/Meth. } & \multirow[b]{2}{*}{ Bott 1} & \multirow{2}{*}{\multicolumn{2}{|c|}{ Number }} & \multirow[b]{3}{*}{ Mean } & \multicolumn{2}{|c|}{ Standard Deviation } & \multirow{3}{*}{$\begin{array}{r}F \\
\text { ratio } \\
\end{array}$} \\
\hline & & & & & Bottle & Error. & \\
\hline & 1 & 2 & 3 & & $\underline{2 \mathrm{df}}$ & $3 \mathrm{df}$ & \\
\hline \multicolumn{8}{|c|}{$\mathrm{Nb}$} \\
\hline \multirow[t]{2}{*}{ WHOI/XRF } & 9.4 & 9.0 & 8.8 & 9.0 & Neg. & 0.5 & $0.18 \mathrm{NS}$ \\
\hline & 8.3 & 8.8 & 9.5 & & & & \\
\hline \multirow[t]{2}{*}{ UwUrz/XRF } & 8 & 7 & 7 & 7.5 & .4 & .4 & $3.00 \mathrm{NS}$ \\
\hline & 8 & 7 & 8 & & & & \\
\hline \multirow[t]{3}{*}{$\mathrm{Birm} / \mathrm{XRF}$} & 5.8 & 5.7 & - & 5.9 & Neg. & .39 &. $\mathrm{OL}_{4} \mathrm{NS}$ \\
\hline & 6.0 & 5.5 & - & & & & \\
\hline & 5.7 & 6.5 & - & & & & . \\
\hline \multirow[t]{3}{*}{ Nott/XRF } & 11.70 & 9.84 & - & 10.22 & Neg. & 1.36 & .13NS \\
\hline & 9.64 & 11.65 & - & & & & \\
\hline & 9.91 & 8.55 & - & & & & \\
\hline \multirow[t]{2}{*}{ Tohok/IPAA } & 4.8 & 5.8 & 5.9 & 5.5 & .2 & .4 & $1.55 \mathrm{NS}$ \\
\hline & 504 & 5.7 & 5.2 & & & & \\
\hline HMI/SSMS & 8.19 & 7.4 & - & 7.29 & Neg. & 1.2 & $.07 \mathrm{NS}$ \\
\hline
\end{tabular}

$\begin{array}{llllllll}\text { HMI/SSMS } & 8.19 & 7.4 & - & 7.29 & \text { Neg. } & 1.2 & .07 N S\end{array}$ $7.69 \quad 8.4 \quad-$

$6.38 \quad 5.7 \quad-$ $\begin{array}{lllllllll}\text { USGSR/Spph } & 9.0 & 9.6 & 9.2 & 9.38 & <.000 & .28 & 1.00 \mathrm{NS}\end{array}$ $9.7 \quad 9.6 \quad 9.2$

$\begin{array}{lllllllll}\mathrm{ETH} / \mathrm{XRF} & 9 & 9 & 9 & 9 & - & 9 & -\end{array}$

\begin{tabular}{llllllll}
\hline Birm/XRF & 12.7 & 13.4 & - & 12.4 & Neg. & .62 & $.21 N S$ \\
& 12.2 & 12.1 & - & & & &
\end{tabular}
$11.9 \quad 12.0$

$\begin{array}{lllllllll}\text { NERF/INAA } & 28 & 23 & 27 & 23 & \text { Neg. } & 4.4 & .66 \mathrm{NS}\end{array}$ $\begin{array}{lllrllll} & 23 & 18 & 19 & & & & \\ \text { Open/INAA } & 14.8 & 4.1 & - & 14.3 & .70 & .90 & 2.82 N S\end{array}$ $\begin{array}{lll}15.7 & 12.5 \quad-\end{array}$

$\begin{array}{llllllll} & 1.1 & 4.3 & - & & & & \\ \text { USGSR/INAA } & 16 & 13 & 12 & 13.3 & .9 & 1.3 & 1.90 \mathrm{NS}\end{array}$ $\begin{array}{llllllll} & 13 & 14 & 12 & & & & \\ \text { Chels/INAA } & 13.3 & 13.4 & - & 13.47 & \text { Neg. } & .94 & .07 N S\end{array}$ $\begin{array}{llll}12.1 & 13.4 & - \\ 14.7 & 13.9 & -\end{array}$ $\begin{array}{lrrrrrrr}\text { ETH/XRP } & 6 & 9 & 12 & 9 & - & - & - \\ \text { Kjel1/IDMS } & 12 & - & 9 & 10.5 & - & - & -\end{array}$

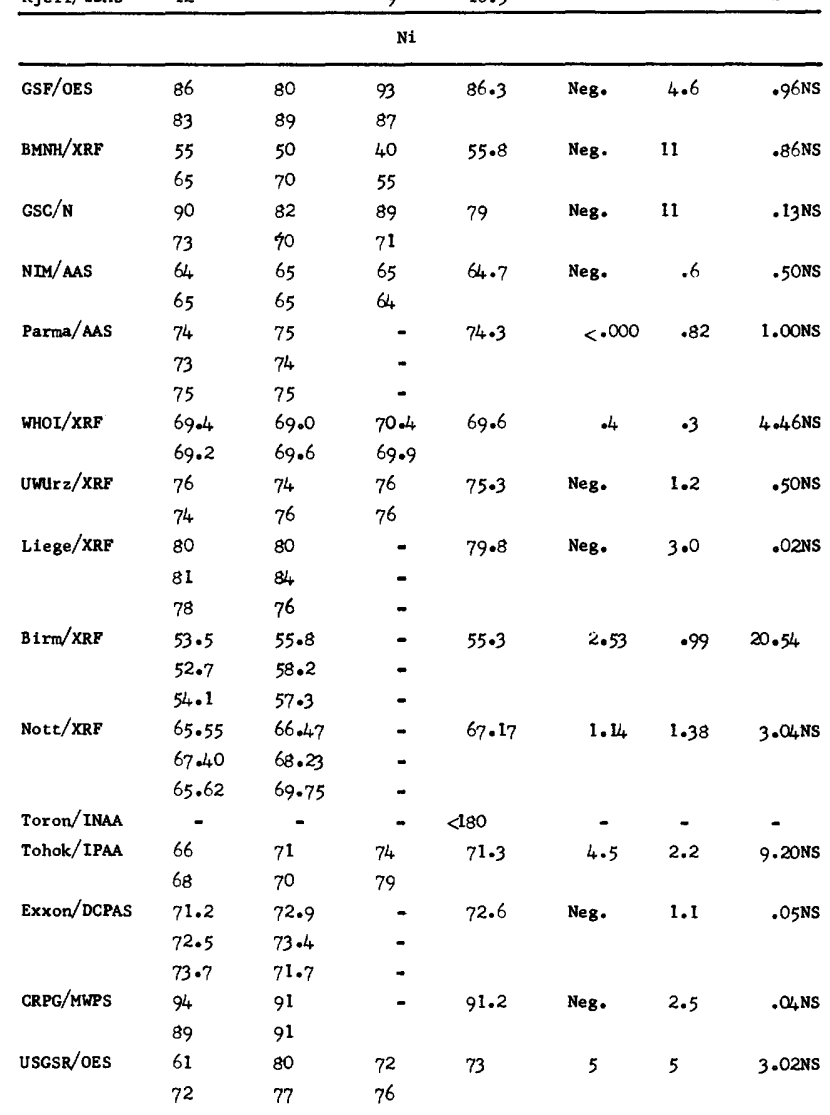


Table 1. Analytical data for USGS-W-2 (cont.)

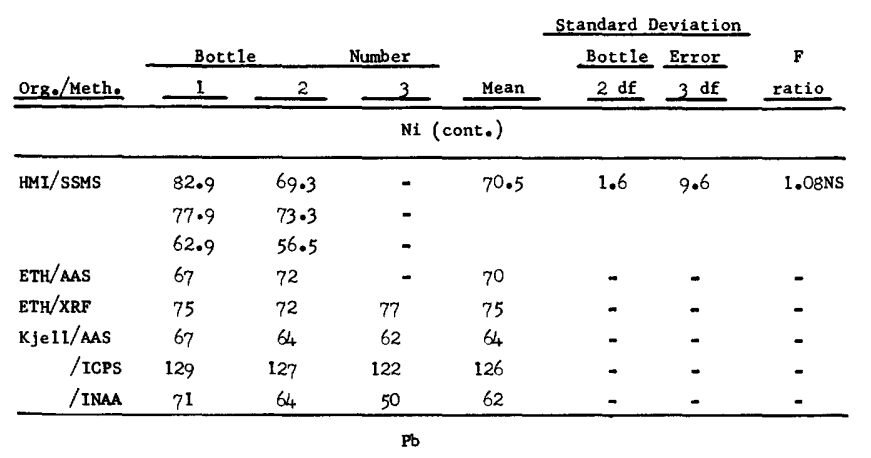

\begin{tabular}{lccccccc}
\hline GSF/IDMS & - & - & - & 7.66 & - & - & - \\
B IO/AAS & - & - & - & $<10$ & - & - & - \\
UWUrz/XRF & 6 & 5 & 7 & 6.3 & $<.00$ & .8 & $1.00 N$ \\
& 6 & 7 & 7 & & & & \\
Birm/ XRF & 10.0 & 11.5 & - & 10.0 & Neg. & 1.91 & $.77 \mathrm{NS}$ \\
& 12.8 & 7.9 & - & & & & \\
& 9.2 & 8.5 & - & & & & \\
ETH/XRF & 14 & 15 & 16 & 15 & - & - & - \\
\hline
\end{tabular}

\begin{tabular}{llllllll}
\multicolumn{8}{c}{$\mathrm{Rb}$} \\
\hline $\mathrm{GSC} / \mathrm{X}$ & 15 & $<10$ & 15 & 15 & - & -
\end{tabular}

$\begin{array}{rrrrrrrr} & 17 & <10 & 16 & & & - & - \\ \text { GSC/M* } & 22 & 21 & 14 & 20 & \text { Neg. } & 4.1 & .19 N S\end{array}$

$\begin{array}{llllllll} & 21 & 20 & 24 & & \text { Neg. } & 4.1 & .19 N S \\ \text { B IO/AAS } & 21 & 21 & 20 & 21 & \text { Neg. } & 1.2 & .50 N S\end{array}$

$\begin{array}{llllllll} & 23 & 21 & 22 & & & & \\ \text { WHOI } / \mathrm{XRF} & 21.5 & 20.5 & 22.3 & 21.1 & \text { Neg. } & .8 & .54 \mathrm{NS}\end{array}$

$\begin{array}{llllllll} & 21.1 & 20.6 & 20.3 & & & & \\ \text { UWUIz } / \text { XRF } & 20 & 20 & 20 & 20.2 & <.00 & .4 & 1.00 \mathrm{NS}\end{array}$

\begin{tabular}{|c|c|c|c|c|c|c|c|}
\hline & & & & & & $4+4$ & 100020 \\
\hline & 20 & 20 & 21 & & & & \\
\hline WSU/XRF & 19 & 17 & 19 & 15.7 & Neg. & 4.1 & $.01 N S$ \\
\hline
\end{tabular}

$\begin{array}{llllllll}\text { Liege/XRF } & 23 & 20 & - & 22.2 & \text { Neg. } & 1.5 & .64 \mathrm{NS}\end{array}$

$\begin{array}{llllllll} & 21 & 22 & - & & & & \\ & 24 & 23 & - & & & & \\ \text { Birm/XRF } & 19.9 & 20.5 & - & 20.3 & \text { Neg. } & .60 & .07 N S\end{array}$

$\begin{array}{llllllll} & 21.3 & 20.4 & - & & & & \\ & 20.0 & 19.9 & - & & & & \\ \text { Nott/XRF } & 20.40 & 19.85 & - & 20.30 & \text { Neg. } & .55 & .83 N S\end{array}$

$\begin{array}{llllllll} & 20.21 & 21.22 & - & & & & \\ & 19.67 & 20.45 & - & & & & \\ \text { Toron/INAA } & 20 & 33 & 26 & 35.6 & 2.4 & 10.9 & 1.14 \mathrm{NS}\end{array}$

$\begin{array}{llllllll}\text { Toron/INAA } & 20 & 33 & 26 & 35.6 & 2.4 & 10.9 & 1.14 \mathrm{NS} \\ & 20 & 47 & 40 & & & & \\ & 44 & 43 & 47 & & & & \end{array}$

$\begin{array}{llllllll}\text { HMI/INAA-W } & 17.1 & 18.8 & - & 17.8 & \text { Neg. } & 1.2 & .48 \mathrm{NS}\end{array}$

$\begin{array}{lllllllll} & 18.1 & 17.5 & - & & & & \\ & 19.2 & 16.0 & - & & & & \\ \text { HMI/INAA-B } & 21.0 & 22.0 & - & 23.5 & \text { Neg. } 4.1 & .79 \mathrm{NS}\end{array}$

$\begin{array}{llllllll} & 27.0 & 29.0 & - & & & & \\ & 18.0 & 24.0 & - & & & & \\ \text { LASL/INAA-1 } & 43 & 19 & - & 24.3 & 4.4 & 9.6 & 1.64 \mathrm{NS}\end{array}$

$\begin{array}{llllllll}\text { LASL/INAA-1 } & 43 & 19 & - & 24.3 & 4.4 & 9.6 & 1.64 \mathrm{NS}\end{array}$

$\begin{array}{llllllll}\text { USGSR/INAA } & 21 & 20 & 25 & 23.0 & \text { Neg. } & 2.5 & .7 \text { INS }\end{array}$

$\begin{array}{llllllll}\text { TOHOK/IPAA } & 18 & \text { I9 } & 18 & 19 & \text { Neg. } & 1.3 & .30 N S\end{array}$

$\begin{array}{llllllll}\text { UInd/Flph } & 17 & 20 & 18 & 18.8 & \text { Neg. } & 1.2 & .78 \mathrm{NS}\end{array}$

$\begin{array}{llllllll}\mathrm{CRPG} / \mathrm{Fl} 1 \mathrm{ph} & 20 & 18 & - & 19.8 & \mathrm{Neg} . & 2 & .06 \mathrm{NS}\end{array}$

$\begin{array}{llllllll} & 19 & 22 & - & & & \\ \text { USGSR/OES } & 12 & 14 & 11 & 12 & 1.4 & .6 & 13.50\end{array}$

$\begin{array}{llllllll} & 12 & 13 & 10 & & & & \\ \text { HMI/SSMS } & 15.3 & 20.3 & - & 15.2 & \text { Neg. } & 2.8 & .45 \mathrm{NS}\end{array}$

$\begin{array}{lllll} & 13.9 & 12.8 & - & \\ \mathrm{ETH} / \mathrm{XRF} & 23 & 22 & 24 & 23\end{array}$

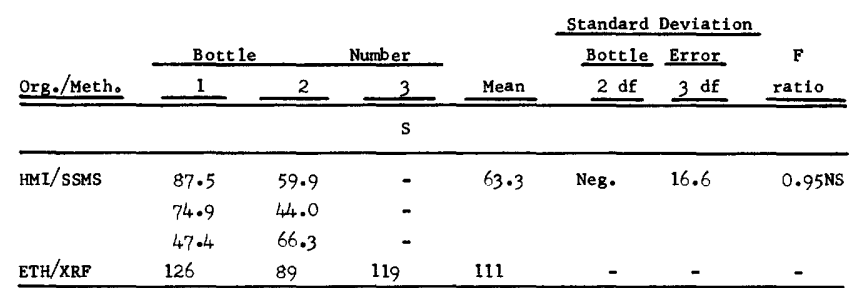

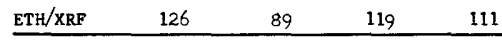

\begin{tabular}{lccccccc}
\hline GSC/c & .7 & .7 & .7 & .7 & - & - & - \\
Toron/INAA & .7 & .7 & .7 & & & & \\
& .76 & .75 & .89 & .666 & Neg. & .16 & .34 NS \\
& .54 & .68 & .55 & & & & \\
HMI/INAA-W & .71 & .40 & .71 & & & & \\
& .95 & .95 & - & 1.01 & .065 & .088 & 2.64 NS \\
& .89 & 1.11 & - & & & & \\
HMI/INAA-B & 1.02 & 1.15 & - & & & & \\
& .90 & 1.07 & - & 1.06 & .125 & .095 & $6.18 N S$ \\
& .91 & 1.23 & - & & & & \\
NERF/INAA & 1.09 & 1.18 & - & & & & \\
& .17 & .21 & - & .19 & - & - & - \\
LASL/INAA-I & .18 & .20 & .20 & & & & \\
& .76 & .69 & - & .85 & Neg. & .12 & $<.00 N S$ \\
& .85 & .94 & - & & & &
\end{tabular}

\begin{tabular}{llllllll} 
& .8 & .7 & .8 & & & & \\
Kjell/INAA & .72 & .68 & .74 & .71 & - & - & - \\
\hline
\end{tabular}

$\begin{array}{llllllll}\text { GSF/OES } & 41 & 40 & 52 & 41.0 & \text { Neg. } & 10.5 & .13 \mathrm{NS}\end{array}$

$\begin{array}{lllllll}\text { NIM/ INAA } & 33.3 & 34.1 & 34.9 & 34.8 & \text { Neg. } & 1.1\end{array}$

$\begin{array}{llllllll} & 35.8 & 35.2 & 35.2 & & & & \\ \text { Toron/INAA } & 34.8 & 34.1 & 33.2 & 34.01 & \mathrm{Neg} . & 1.04 & .16 \mathrm{NS}\end{array}$

$\begin{array}{lll}33.3 & 34.9 & 34.8 \\ 34.3 & 32.2 & 34.5\end{array}$

HMI/INAA-W $35.3 \quad 37.6 \quad-6 \quad 36.0 \quad$ Neg. $1.4<.00 N$

$36.5 \quad 34.0$

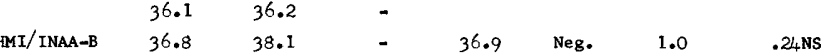

$\begin{array}{llll}37.0 & 35.4 & - & \\ 37.6 & 36.7 & -\end{array}$

$\begin{array}{llllllll}\text { NERF/INAA } & 36.8 & 36.7 & 36.0 & 36.7 & .55 & .29 & 7.92 \mathrm{NS}\end{array}$

LASL/INAA- $138 \quad 37 \quad$ - $37 \quad \begin{array}{llllll} & 37.5 & .7 & 0 & *\end{array}$

$\begin{array}{llllllll} & 38 & 37 & - & & & & \\ \text { Open/ INAA } & 36.6 & 35.2 & - & 35.55 & \text { Neg. } & .89 & .93 \text { NS }\end{array}$

$\begin{array}{lccccccc} & 36.4 & 34.5 & - & & & & \\ \text { USGSR/INAA } & 36.0 & 35.8 & 32.7 & 34.4 & .92 & 1.30 & 1.99 \mathrm{NS}\end{array}$

$\begin{array}{lllclllll} & 32.9 & 35.5 & 33.4 & & & & & \\ \text { LASL/INAA } & 35.9 & 35.3 & - & 35.6 & \text { Nego } & .69 & .59 \mathrm{NS}\end{array}$

$\begin{array}{llllllll} & 35.3 & 36.7 & - & & & & \\ & 34.8 & 35.3 & - & & & & \\ \text { USGSR/OES } & 50 & 51 & 48 & 49.8 & 2.2 & 1.9 & 3.76 \mathrm{NS}\end{array}$

$\begin{array}{llllllll}\text { HMI/SSMS } & 54 & 50 & 46 & & & & \\ & 38.2 & 33.9 & - & 29.4 & \text { Neg. } & 8.2 & \text {.48NS }\end{array}$

\begin{tabular}{llllllll} 
& 19.5 & 24.7 & - & & & & \\
$\mathrm{ETH} / \mathrm{XRF}$ & 36 & 35 & 36 & 36 & - & - & - \\
$\mathrm{KjeI1} / \mathrm{INAA}$ & 32.6 & 34.0 & 32.0 & 32.9 & - & - & - \\
\hline
\end{tabular}

\begin{tabular}{lllllllll}
\multicolumn{8}{c}{$\mathrm{Sm}$} \\
\hline NIM/ INAA & 3.2 & 3.4 & 3.4 & 3.37 & $<.00$ & .08 & $1.00 \mathrm{NS}$
\end{tabular}

$\begin{array}{llllllll} & 3.4 & 3.4 & 3.4 & & & \\ \text { Toron/INAA } & 2.64 & 3.11 & 2.76 & 2.87 & .067 & .136 & 1.74 \mathrm{NS}\end{array}$

$2.76 \quad 2.90 \quad 3.00$

$2.85 \quad 2.80 \quad 3.00$

HMI/INAA-W $\quad 3.73 \quad 3.78 \quad-\quad 3.71 \quad 3$ Neg. $\quad 3.11<.0 O N S$ $3.69 \quad 3.54$ 
Table 1. Analytical data for USGS-W-2 (cont.)

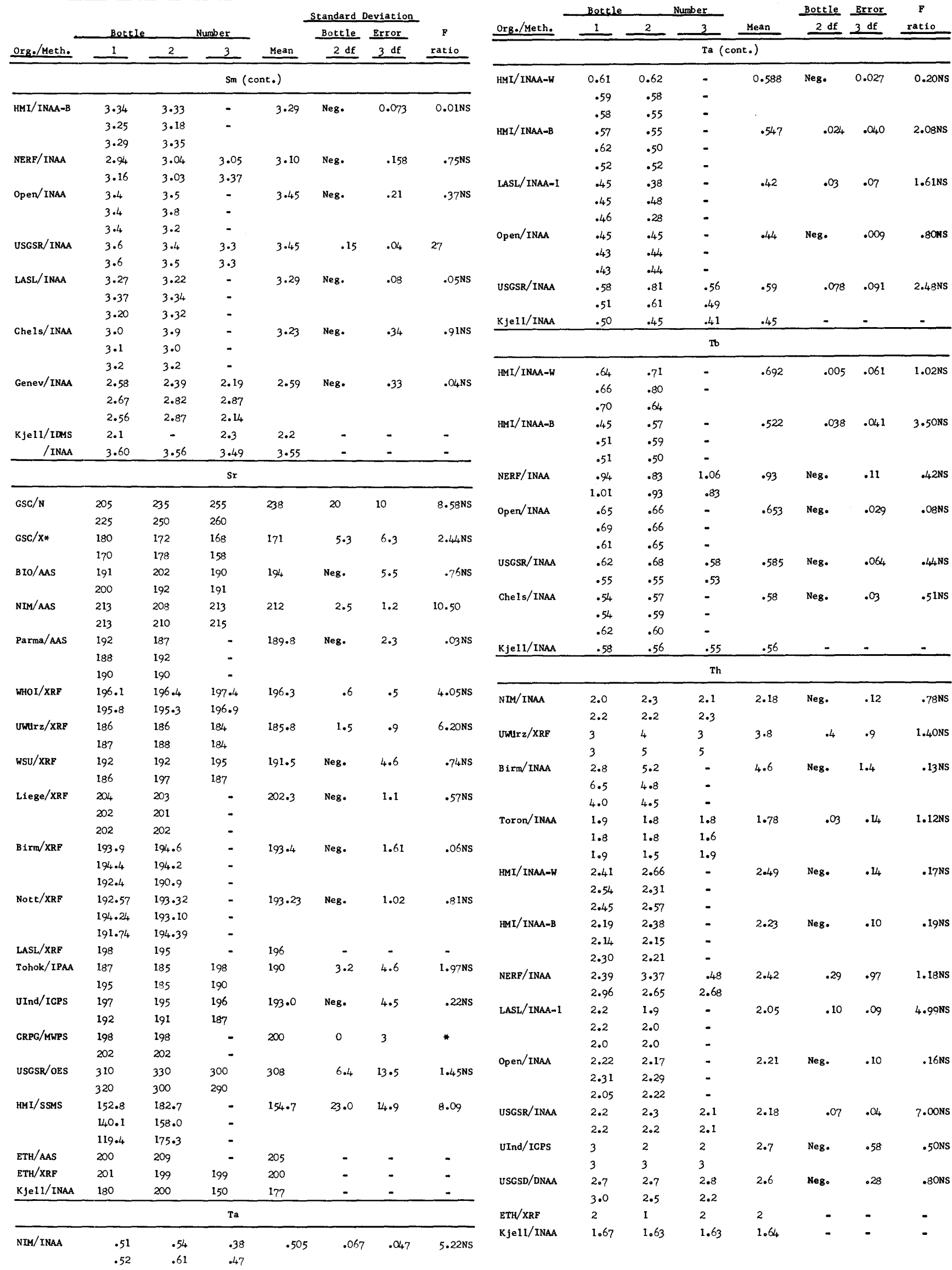


Table 1. Analytical data for USGS-W-2 (cont.)

\begin{tabular}{|c|c|c|c|c|c|c|c|}
\hline \multirow[b]{2}{*}{ Org./Meth. } & \multicolumn{2}{|c|}{ Bottle } & Number & \multirow[b]{2}{*}{ Mean } & $\begin{array}{l}\text { tandard D } \\
\text { Botelle }\end{array}$ & $\begin{array}{l}\text { Deviation } \\
\text { Error }\end{array}$ & \multirow{2}{*}{$\begin{array}{c}\mathbf{F} \\
\text { ratio } \\
\end{array}$} \\
\hline & 1 & 2 & 3 & & $\underline{2 \mathrm{df}}$ & $3 \mathrm{df}$ & \\
\hline \multicolumn{8}{|c|}{$\mathbf{T} \mathbf{i}$} \\
\hline \multirow[t]{2}{*}{ GSF/OES } & 8100 & 6800 & 6500 & 6933 & 299 & 532 & $1.63 \mathrm{NS}$ \\
\hline & 6800 & 6900 & 6500 & & & & \\
\hline \multirow[t]{2}{*}{ USGSR/OES } & 760 & 670 & 700 & 785 & 92 & 155 & $1.70 \mathrm{NS}$ \\
\hline & 1100 & 620 & 860 & & & & \\
\hline \multirow[t]{3}{*}{ HMI/SSMS } & 3804 & 3373 & - & 3885 & Neg. & 647 & $.02 \mathrm{NS}$ \\
\hline & 4528 & 4505 & - & & & & \\
\hline & 3095 & 3904 & - & & & & \\
\hline
\end{tabular}

\begin{tabular}{llllllll}
\hline USGSR/AAS & .16 & .20 & .16 & .165 & $\mathrm{Neg}$. & .020 & $.36 \mathrm{NS}$ \\
& .16 & .15 & .16 & & & & \\
\hline
\end{tabular}

\begin{tabular}{|c|c|c|c|c|c|c|c|}
\hline Open/INAA & .43 & .43 & - & .44 & .02 & $\cdot \mathrm{C}_{3}$ & $2.44 \mathrm{NS}$ \\
\hline & .43 & .50 & - & & & & \\
\hline
\end{tabular}

$\begin{array}{llllllll} & .39 & .44 & - & & & & \\ \text { USGSR/INAA } & .36 & .38 & .27 & .30 & .06 & .015 & 3.69 \mathrm{NS}\end{array}$

$\begin{array}{llllllll}\text { Chels/INAA } & .28 & .34 & .19 & & & & \\ & .34 & .39 & - & .39 & \text { Neg. } & .04 & .09 N S\end{array}$

\begin{tabular}{lll}
.36 & .40 & - \\
.45 & .39 & - \\
\hline U
\end{tabular}

\begin{tabular}{llllllll}
\hline Toron/ INAA & 1.5 & 1.8 & 2.5 & 1.89 & .16 & .32 & $1.78 \mathrm{NS}$ \\
& 2.0 & 1.6 & 2.2 & & & &
\end{tabular}

$\begin{array}{lccccccc} & 2.2 & 1.5 & 1.7 & & & & \\ \text { LASL/DNAA-1 } & .59 & .59 & - & .61 & .04 & .03 & 5.40 \mathrm{NS}\end{array}$

$\begin{array}{lllllllll} & .67 & .58 & - & & & & & \\ & .66 & .57 & - & & & & \\ \text { USGSR/INAA } & .5 & .6 & .5 & .52 & .07 & .04 & 7.00 \mathrm{NS}\end{array}$

$\begin{array}{llllllll} & .5 & .6 & .4 & & & & \\ \text { *TeXA\&M/ DNAA } & .45 & .43 & - & .416 & .013 & .051 & 1.42 \mathrm{NS}\end{array}$

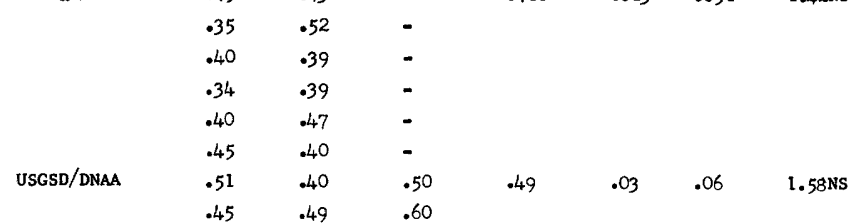

\begin{tabular}{llllllll}
$\mathrm{ETH} / \mathrm{XRF}$ & $1^{.45}$ & $1^{.49}$ & $1^{.60}$ & 1 & - & - & - \\
$\mathrm{Kjel1} / \mathrm{INAA}$ & .55 & .51 & .53 & .53 & - & - & - \\
\hline
\end{tabular}

\begin{tabular}{llllllll}
\hline \multicolumn{8}{c}{$v$} \\
\hline GSF/OES & 270 & 260 & 250 & 260 & 0 & 11.5 & $*$
\end{tabular}

$\begin{array}{lllllllll} & 250 & 260 & 270 & & & & & \\ \text { GSC/N } & 280 & 280 & 290 & 267 & \text { Neg. } & 24 & .14\end{array}$

\begin{tabular}{|c|c|c|c|c|c|c|c|}
\hline & & - & $\infty 7$ & 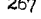 & Neg. & 4 & OINSS \\
\hline & 260 & 240 & 250 & & & & \\
\hline B IO/AAS & 254 & 244 & 255 & 253 & Neg. & 6 & $.39 \mathrm{NS}$ \\
\hline
\end{tabular}

\begin{tabular}{llllllll} 
& & & & \\
\multirow{2}{*}{ NIM/ICPS } & 250 & 258 & 257 & 253 & Neg. & 6 & $.39 \mathrm{NS}$ \\
& 298 & 332 & 290 & 319.7 & Neg. & 23 & $.74 \mathrm{NS}$
\end{tabular}

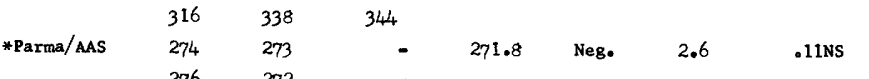

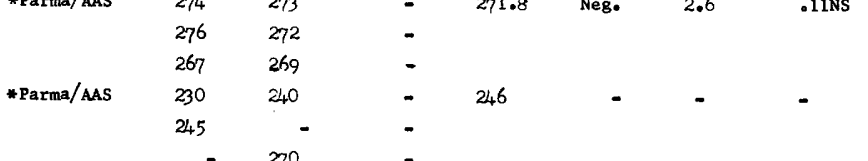

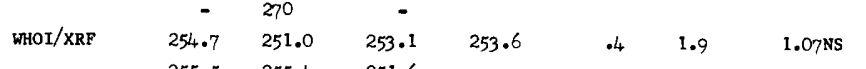

$\begin{array}{lllclllll}\text { Liege/XRF } & 255.5 & 255.4 & 251.6 & & & & \\ & 246 & 24 \mathrm{I} & - & 249.0 & \text { Neg. } & 5.2 & .22 \mathrm{NS}\end{array}$

\begin{tabular}{llllllll} 
& 252 & 249 & - & & & & \\
\multirow{5}{*}{ Nott/XRF } & 252 & 254 & - & & & & \\
& 245.54 & 242.09 & - & 245.55 & Neg. & 4.18 & $.05 \mathrm{NS}$ \\
& 240.11 & 248.15 & - & & & & \\
& 249.84 & 247.58 & - & & & & \\
Toron/INAA & 259 & 263 & 283 & 270.4 & Neg. & 10.4 & $.75 \mathrm{NS}$ \\
& 266 & 282 & 261 & & & & \\
& 272 & 264 & 284 & & & &
\end{tabular}

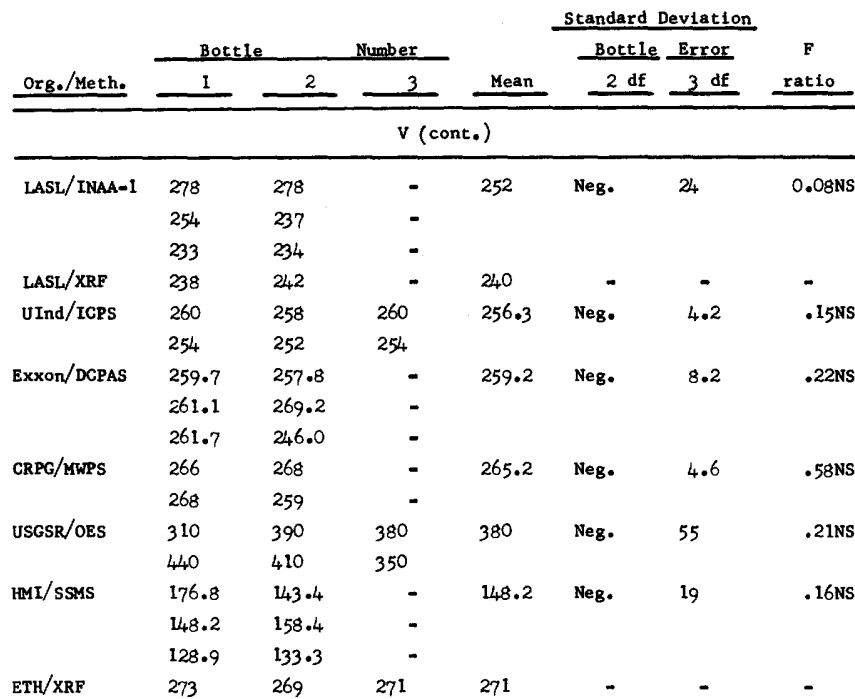

\begin{tabular}{llllllll}
\hline \multicolumn{10}{c}{ W } \\
\hline USGSR/Spph & .31 & .26 & .23 & .263 & .011 & .024 & $1.44 \mathrm{NS}$
\end{tabular}

\begin{tabular}{lllllllll}
\hline \multicolumn{7}{c}{} & .26 & .26 \\
\hline GSF/OES & 31 & 33 & 37 & 29.8 & Neg. & 6.3 & .08 NS \\
& 26 & 29 & 23 & & & &
\end{tabular}

$\begin{array}{llllllll}\text { WHOI/XRF } & 24.4 & 23.7 & 23.5 & 24.1 & \text { Neg. } & .6 & .07 N S\end{array}$

$\begin{array}{llllllll}\text { UWUrz } / \text { XRF } & 24.0 & 24.6 & 24.5 & & & & \\ 20 & 24 & 24 & 23 & 2.6 & .6 & 40.5\end{array}$

$\begin{array}{llllllll} & & & & & & & \\ \text { WSU/XRF } & 18 & 17 & 25 & 16.7 & \text { Neg. } & 3.1 & .28 N S\end{array}$

$\begin{array}{llllllll} & 14 & 15 & 15 & & & & \\ \text { Liege/XRF } & 23 & 24 & - & 21.2 & \text { Neg. } & 2.2 & .014 \mathrm{NS}\end{array}$

$\begin{array}{llllllll} & 21 & 19 & - & & & & \\ \text { B irm } / \text { XRF } & 20.3 & 20.5 & - & 20.1 & \text { Neg. } & .59 & .31 \mathrm{NS}\end{array}$

$\begin{array}{lllllllll} & 20.0 & 19.4 & - & & & & \\ \text { Nott } / \text { XRF } & 23.89 & 25.46 & - & 24.48 & \text { Neg. } & .61 & & \end{array}$

$\begin{array}{lll}24.48 & 23.96 & - \\ 24.62 & 24.46 & -\end{array}$

$\begin{array}{llllllll}\text { Tohok/IPAA } & 26 & 29 & 28 & 26.2 & \text { Neg. } & 3.0 & .24 \mathrm{NS}\end{array}$

$\begin{array}{llllllll}\text { UInd/ICPS } & 23 & 22 & 23 & 22.3 & \text { Neg。 } & .58 & .50 \mathrm{NS}\end{array}$

$\begin{array}{llllllll}\text { USGSR/OES } & 32 & 35 & 30 & 32.5 & 2.2 & .9 & 12.60\end{array}$

$\begin{array}{lllllllll}\text { HML/SSMS } & 42.3 & 25.5 & - & 28.6 & 4.3 & 7.8 & 1.89 \mathrm{NS}\end{array}$

$\begin{array}{rrrr}35.8 & 23.4 & - & \\ & 20.8 & 23.6 & -\end{array}$

\begin{tabular}{llllllll}
$\mathrm{ETH} / \mathrm{XRF}$ & 23 & 23 & 24 & 23 & - & - & - \\
\hline & \multicolumn{8}{c}{} & & \\
\hline
\end{tabular}

$\begin{array}{lllllllll}\mathrm{NIM} / \mathrm{INAA} & 2.7 & 2.3 & 2.7 & 2.55 & \mathrm{Neg} . & .33 & .1_{4} \mathrm{NS}\end{array}$

$\begin{array}{llllllll}\text { Toron/INAA } & 1.9 & 2.6 & 2.2 & 2.14 & \text { Neg. } & .33 & .48 \mathrm{NS}\end{array}$

$\begin{array}{llllllll} & 2.1 & 1.8 & 2.7 & & & & \\ \text { HMI/INAA-W } & 2.08 & 2.21 & - & 2.12 & \text { Neg. } & .069 & .01 \mathrm{NS}\end{array}$

$\begin{array}{llllllll}2.08 & 2.21 & - & 2.12 & \text { Neg. } & .069 & .01 \mathrm{NS} \\ 2.18 & 2.05 & - & & & & \\ 2.11 & 2.09 & - & & & & \end{array}$

$\begin{array}{lllllllll}\text { HMI/INAA-B } & 2.10 & 2.06 & - & 2.08 & \text { Neg. } & .034 & .72 \mathrm{NS}\end{array}$

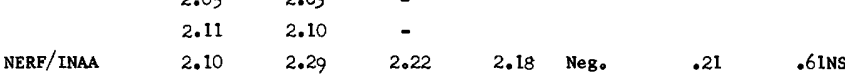

$\begin{array}{lllllllll} & 2.28 & 1.82 & 2.36 & & & & \\ \text { Open/INAA } & 2.13 & 2.13 & - & 2.09 & \mathrm{Neg} . & .03 & \text {.06NS } \\ & 2.07 & 2.08 & - & & & & \end{array}$ 
Table 1. Analytical data for USGS-W-2 (cont.)

\begin{tabular}{|c|c|c|c|c|c|c|c|}
\hline \multirow[b]{3}{*}{ Org./Meth. } & \multirow{3}{*}{$\begin{array}{c}\text { Bottle } \\
\frac{1}{1}\end{array}$} & \multirow{2}{*}{\multicolumn{2}{|c|}{ Number }} & \multirow[b]{3}{*}{ Mean } & \multicolumn{2}{|c|}{ Standard Deviation } & \multirow{3}{*}{$\begin{array}{c}\mathbf{F} \\
\text { ratio } \\
\end{array}$} \\
\hline & & & & & Bottle & Error & \\
\hline & & 2 & 3 & & $\underline{2 \mathrm{df}}$ & $3 \mathrm{df}$ & \\
\hline \multicolumn{8}{|c|}{$\mathrm{Yb}$ (cont.) } \\
\hline \multirow[t]{3}{*}{ Chels/INAA } & 2.14 & 2.21 & - & 2.10 & Neg. & 0.08 & $0.36 \mathrm{NS}$ \\
\hline & 2.03 & 2.01 & - & & & & \\
\hline & 2.06 & 2.13 & - & & & & \\
\hline \multirow[t]{2}{*}{ USGSR/INAA } & 2.2 & 2.1 & 1.9 & 2.05 & .09 & .07 & $4.00 \mathrm{NS}$ \\
\hline & 2.1 & 2.0 & 2.0 & & & & \\
\hline \multirow[t]{3}{*}{ LASL/INAA } & 2.00 & 2.05 & - & 2.07 & Neg. & .12 & $.53 \mathrm{NS}$ \\
\hline & 2.06 & 2.30 & - & & & & \\
\hline & 2.04 & 1.97 & - & & & & \\
\hline \multirow[t]{3}{*}{ Genev/INAA } & 1.64 & 2.08 & 2.22 & 2.00 & Neg. & .32 & $.02 \mathrm{NS}$ \\
\hline & 2.30 & 1.94 & 1.83 & & & & \\
\hline & 2.15 & 1.88 & 2.07 & & & & \\
\hline \multirow[t]{2}{*}{ USGSR/OES } & 4 & 3 & 3 & 3.2 & $<.00$ & .4 & $1.00 \mathrm{NS}$ \\
\hline & 3 & 3 & 3 & & & & \\
\hline Kjel1/IDens & 2.6 & - & 3.1 & 2.8 & - & - & - \\
\hline /INAA & 1.93 & 1.86 & 1.91 & 1.90 & - & - & - \\
\hline
\end{tabular}

\begin{tabular}{|c|c|c|c|c|c|c|c|}
\hline \multirow[t]{2}{*}{ B IO/AAS } & 76 & 78 & 78 & 77 & Neg. & 1.4 & $.36 \mathrm{NS}$ \\
\hline & 77 & 77 & 75 & & & & \\
\hline \multirow[t]{2}{*}{ NIM/AAS } & 81 & 82 & 84 & 83.2 & .8 & 1.7 & $1.47 \mathrm{NS}$ \\
\hline & 82 & 86 & 84 & & & & \\
\hline \multirow[t]{2}{*}{ WhOI/XRF } & 70.8 & $71 \cdot 3$ & 72.7 & 71.4 & .6 & .5 & $4.62 \mathrm{NS}$ \\
\hline & 70.8 & 71.1 & 71.6 & & & & \\
\hline \multirow[t]{2}{*}{ UWUrz/XRF } & 78 & 69 & 74 & 74 & 4.0 & .8 & 48 \\
\hline & 78 & 71 & 74 & & & & \\
\hline \multirow[t]{3}{*}{ Llege/XRF } & 74 & 78 & - & 72.5 & Neg. & 4.0 & $.09 \mathrm{NS}$ \\
\hline & 69 & 73 & - & & & & \\
\hline & 73 & 68 & - & & & & \\
\hline \multirow[t]{3}{*}{ Birm/XRF } & 71.5 & 72.1 & - & 72.1 & Neg. & .76 & $.10 N S$ \\
\hline & 72.5 & 71.0 & - & & & & \\
\hline & 72.5 & 72.8 & - & & & & \\
\hline \multirow[t]{3}{*}{ Nott/XRF } & 83.49 & 82.45 & - & 79.99 & Neg. & 3.08 & $.16 \mathrm{NS}$ \\
\hline & $77 \cdot 43$ & 76.21 & - & & & & \\
\hline & 80.56 & 79.79 & - & & & & \\
\hline \multirow[t]{3}{*}{ Curie/XRF } & 68.95 & 69.28 & - & 68.21 & Neg. & 1.67 & $.03 \mathrm{NS}$ \\
\hline & 66.61 & 66.01 & - & & & & \\
\hline & $69 \cdot 43$ & 69.00 & - & & & & \\
\hline \multirow[t]{2}{*}{ USGSR/INAA } & 88 & 90 & 76 & 84.3 & $4 \cdot 2$ & 3.4 & $4.04 \mathrm{NS}$ \\
\hline & 83 & 87 & 82 & & & & \\
\hline LASL/XRF & 97 & 95 & - & 96 & - & - & - \\
\hline \multirow[t]{2}{*}{ UInd/ICPS } & 77 & 77 & 76 & 76.3 & Neg. & .58 & .5 ONS \\
\hline & 76 & 76 & 76 & & & & \\
\hline \multirow[t]{3}{*}{ Exxon/DCPAS } & $79 \cdot 3$ & 79.1 & - & $79 \cdot 7$ & 1.3 & 3.1 & $1.56 \mathrm{NS}$ \\
\hline & 78.4 & $77 \cdot 5$ & - & & & & \\
\hline & 86.2 & $77 \cdot 9$ & - & & & & \\
\hline \multirow[t]{2}{*}{ USGSR/OES } & 66 & 76 & 59 & 59.8 & Neg. & 15 & $.83 \mathrm{NS}$ \\
\hline & 32 & 61 & 65 & & & & \\
\hline \multirow[t]{3}{*}{ HMI/SSMS } & 65.3 & 91.3 & - & 78.9 & 4.2 & 13.2 & $1.31 \mathrm{NS}$ \\
\hline & 93.1 & 82.3 & - & & & & \\
\hline & 59.6 & 81.5 & - & & & & \\
\hline \multirow[t]{2}{*}{ GCL/AAS } & 80.0 & 82.5 & 82.5 & 81.2 & Neg. & 2.3 & $.60 \mathrm{NS}$ \\
\hline & 82.5 & 77.5 & 82.5 & & & & \\
\hline ETH/AAS & 75.5 & 75 & - & $75 \cdot 2$ & - & - & - \\
\hline ETH $/$ XRF & 77 & 77 & 78 & 77 & - & - & - \\
\hline $\mathrm{Kjel1/AAS}$ & 73 & 72 & 72 & 72 & - & - & - \\
\hline \multirow[t]{2}{*}{ /ICPS } & 60 & 65 & 55 & 60 & - & - & - \\
\hline & & & $z \mathbf{r}$ & & & & \\
\hline \multirow[t]{2}{*}{ GSF/OES } & 99 & 97 & 120 & 96.7 & Neg. & 19.3 & .00 NS \\
\hline & 95 & 96 & 73 & & & & \\
\hline \multirow[t]{2}{*}{$\mathrm{GSC} / \mathrm{N}$} & 81 & 90 & 87 & 82 & Neg. & 6.6 & $.21 \mathrm{NS}$ \\
\hline & 80 & 79 & 75 & & & & \\
\hline \multirow[t]{2}{*}{ WHOI/XRF } & 99.6 & 101.3 & 101.2 & 100.9 & .8 & .3 & 12.78 \\
\hline & 100.2 & 101.1 & 101.7 & & & & \\
\hline \multirow[t]{2}{*}{ UWUr z/XRF } & 106 & 102 & 106 & 104.3 & 2 & .8 & 13.00 \\
\hline & 106 & 102 & 104 & & & & \\
\hline \multirow[t]{2}{*}{ WSU/XRF } & 87 & 84 & 93 & 89.7 & Neg. & 4.0 & $.38 \mathrm{NS}$ \\
\hline & 92 & 92 & 90 & & & & \\
\hline
\end{tabular}

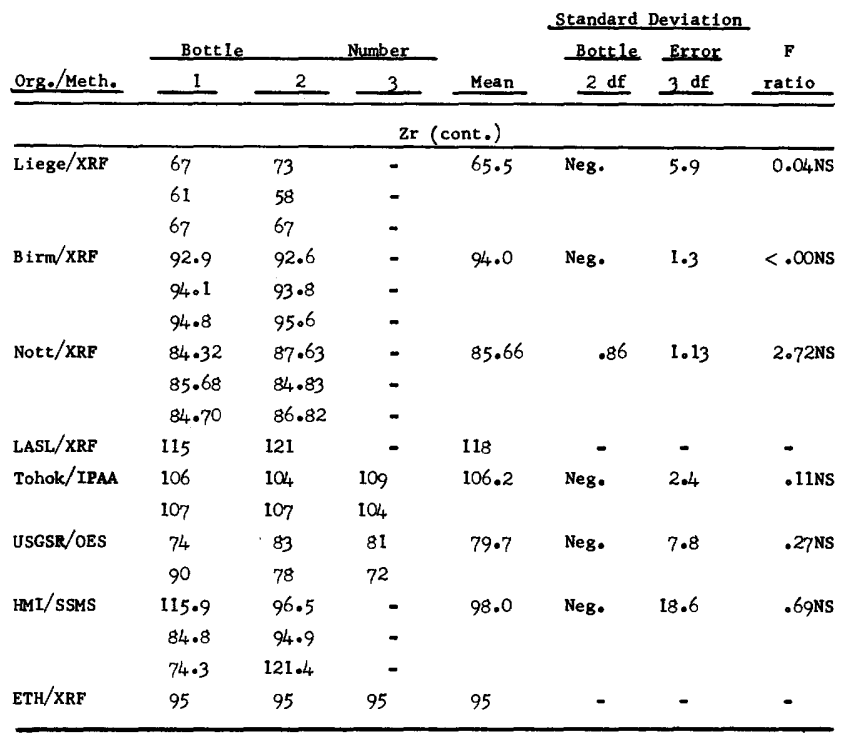


Table 2. Analytical data for USGS-DNC-1

C $\mathrm{SiO}_{2}$ through $\mathrm{Pe}_{2} \mathrm{O}_{3} \mathrm{~T}$ in percent; trace elements in parts per million. Org./Meth,, organization and method. Detalls of methods, where available, are given under the organization name at the end of table 3 . A set of data by an organization whose abbreviation is preceded by an asterisk contains data or estimates explained under the organization name. F ratios noted only by an asterisk could not be calculated because of a zero mean square for bottles or error. Ns, not significant at the $95 \%$ fractile, $F_{0.05}(2,3)=9.55$. Allowable $F$ ratios for other degrees of freedom (df) and probabilities ( $p$ ) are given at the end of table 1 for calculated $F$ ratios not followed by NS. Neg., negative bottle variance. $\mathrm{Fe}_{2} \mathrm{O}_{3} \mathrm{~T}$, total $\mathrm{Fe}$ as $\left.\mathrm{Fe}_{2} \mathrm{O}_{3} \cdot\right]$

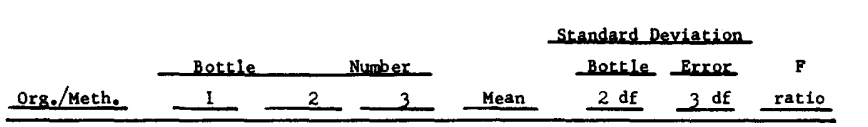

\begin{tabular}{|c|c|c|c|c|c|c|c|}
\hline & & & $\mathrm{SiO}_{2}$ & & & & \\
\hline \multirow[t]{2}{*}{ GSF/Chem. } & 47.46 & 47.41 & 47.40 & $47 \cdot 39$ & Neg. & 0.05 & $0.12 \mathrm{NS}$ \\
\hline & $47 \cdot 35$ & $47 \cdot 37$ & $47 \cdot 36$ & & & & \\
\hline \multirow[t]{2}{*}{ *BMNH/Chem. } & $47 \cdot 32$ & 47.05 & 46.96 & 47.05 & - & - & - \\
\hline & 47.05 & 46.92 & 46.98 & & & & \\
\hline \multirow[t]{2}{*}{ *BMNH/XRF } & $47 \cdot 5$ & $47 \cdot 7$ & 47.8 & 47.57 & Neg. & .21 & $.27 \mathrm{NS}$ \\
\hline & 47.6 & $47 \cdot 3$ & 47.5 & & & & \\
\hline \multirow[t]{2}{*}{ *BMNH/XRF } & 47.19 & 47.27 & 47.06 & 47.06 & Neg. & .22 & $.12 \mathrm{NS}$ \\
\hline & 46.04 & 46.75 & 47.02 & & & & \\
\hline \multirow[t]{2}{*}{$\mathrm{GSC} / \mathrm{A}$} & $47 \cdot 6$ & 47.2 & 47.8 & 47.4 & .20 & .19 & $3.19 \mathrm{NS}$ \\
\hline & $47 \cdot 4$ & $47 \cdot 1$ & $47 \cdot 4$ & & & & \\
\hline \multirow[t]{2}{*}{ GSC/D* } & 47.67 & $47 \cdot 56$ & 47.63 & 47.55 & .06 & $.1 u_{4}$ & $1.35 \mathrm{NS}$ \\
\hline & 47.70 & 47.46 & $47 \cdot 30$ & & & & \\
\hline \multirow[t]{2}{*}{ BIO/AAS } & 47.30 & 47.09 & $47 \cdot 52$ & 47.16 & .28 & .25 & $3.51 \mathrm{NS}$ \\
\hline & 46.87 & 46.66 & 47.52 & & & & \\
\hline \multirow[t]{4}{*}{ *NIM/XRF } & 46.48 & 46.82 & 45.00 & 46.67 & .29 & .58 & $2.00 \mathrm{NS}$ \\
\hline & 46.48 & $47 \cdot 36$ & 46.06 & & & & \\
\hline & 47.13 & 46.82 & 46.93 & & & & \\
\hline & 47.24 & 46.93 & 46.82 & & & & \\
\hline \multirow[t]{2}{*}{$\mathrm{NIM} / \mathrm{IGPS}$} & 46.96 & 46.96 & 46.96 & 46.96 & - & - & - \\
\hline & 46.96 & 46.96 & 46.96 & & & & \\
\hline \multirow[t]{2}{*}{ USGSR/Chem, } & $47 \cdot 4$ & 47.6 & 47.4 & 47.53 & Neg. & .13 & .7ONS \\
\hline & $47 \cdot 7$ & 47.6 & $47 \cdot 5$ & & & & \\
\hline Parma/Chem. & 47.63 & 46.43 & - & 46.58 & - & - & - \\
\hline \multirow[t]{2}{*}{ WHOI/XRF } & 48.56 & 48.23 & 47.89 & $48 \cdot 14$ & .21 & .17 & $3.99 \mathrm{NS}$ \\
\hline & 48.19 & 48.05 & 47.91 & & & & \\
\hline \multirow[t]{2}{*}{$\mathrm{WSU} / \mathrm{XRF}$} & 48.50 & 48.03 & 48.26 & 48.27 & Neg. & .26 &. OgNS \\
\hline & 48.12 & 48.54 & 48.15 & & & & \\
\hline \multirow[t]{2}{*}{ USGSR/XRF } & 48.00 & 47.86 & 47.63 & 48.04 & .11 & .31 & $1.26 \mathrm{NS}$ \\
\hline & 48.45 & 48.42 & 47.89 & & & & \\
\hline \multirow[t]{2}{*}{ *NERF/INAA } & 22.00 & 20.01 & 22.14 & 21.44 & .52 & .60 & $2.47 \mathrm{NS}$ \\
\hline & 21.53 & 21.32 & 21.63 & & & & \\
\hline \multirow[t]{2}{*}{ UInd/ICPS } & 47.0 & 46.5 & 46.7 & 46.75 & .21 & .09 & 11.40 \\
\hline & 47.0 & 46.7 & 46.6 & & & & \\
\hline \multirow[t]{3}{*}{ Exxon/DCPAS } & $47 \cdot 10$ & $47 \cdot 33$ & - & 47.02 & $\cdot 1_{4}$ & .22 & $2.22 \mathrm{NS}$ \\
\hline & 46.73 & 46.88 & - & & & & \\
\hline & 46.83 & 47.24 & - & & & & \\
\hline \multirow[t]{2}{*}{ CRPG/MWPS } & $47 \cdot 15$ & 46.46 & - & 46.85 & Neg. & .56 & $.03 \mathrm{NS}$ \\
\hline & 46.46 & 47.33 & - & & & & \\
\hline ETH/AAS & 46.4 & 46.5 & - & 46.45 & - & - & - \\
\hline $\mathrm{ETH} / \mathrm{XRF}$ & 47.09 & 47.22 & 46.52 & 46.94 & - & - & - \\
\hline \multirow[t]{2}{*}{ *KJe11/XRF } & 21.6 & 21.4 & 21.5 & 21.5 & - & - & - \\
\hline & & & $\mathrm{Al}_{2} \mathrm{O}_{3}$ & & & & \\
\hline GSF/Chemb & $18 \cdot 54$ & 18.54 & 18.45 & 18.54 & Neg. & .05 & $.12 \mathrm{NS}$ \\
\hline
\end{tabular}

Parma Aas

WHOI/XR

UWHrz/XRF

WSU/XRF

USGSR/XRF

*Toron/INAA

the

*NERF/INAA

*LASL/INAA-I

UInd/ICPS

Exxon/DCPAS

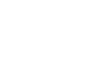

CRPG/MWP

ETH/AAS

ETH/XRF

jel1/Aas

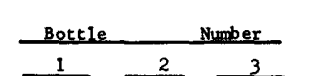

Standard Deviation

$2 \mathrm{df} 3 \mathrm{df}$

$\mathrm{Al}_{2} \mathrm{O}_{3}$ (cont.)

$\begin{array}{lllllll}18.03 & 18.10 & 18.03 & 17.94 & \text { Neg. } & 0.26 & 0.60 \mathrm{NS}\end{array}$

$17.84 \quad 17.50 \quad 18.14$

$\begin{array}{llllllll}18.29 & 18.29 & 17.96 & 18.50 & .05 & .26 & 1.12 \mathrm{NS}\end{array}$

$18.50 \quad 18.50 \quad 18.40$

$18.71 \quad 19.02 \quad 18.60$

$18.71 \quad 18.60 \quad 18.40$

$\begin{array}{lllllll}18.47 & 18.61 & 18.49 & 18.51 & \text { Neg. } & .12 & .11 N S\end{array}$

$18.61 \quad 18.36 \quad 18.53$

$\begin{array}{lll}18.2 & 18.2 & 18.5 \\ 18.4 & 18.4 & 18.4\end{array}$

$17.90 \quad 18.20$

$17.92 \quad 17.83$

$17.80 \quad 18.03$

$19.21 \quad 18.99 \quad 18.82$

$19.03 \quad 18.99 \quad 18.84$

$17.7 \quad 17.9 \quad 17.3$

$\begin{array}{lll}17.4 & 17.6 & 17.4 \\ 18.95 & 18.84 & 18.66\end{array}$

$\begin{array}{lll}18.88 & 18.75 \quad 19.00\end{array}$

$\begin{array}{lll}18.03 \quad 18.02 & 18.77\end{array}$

$18.39 \quad 18.13 \quad 18.46$

$8.89 \quad 9.41 \quad 8.62$

$9.19 \quad 8.56 \quad 8.81$

$\begin{array}{lll}9.19 & 8.56 & 8.81 \\ 8.87 & 9.24 & 8.75\end{array}$

$9.61 \quad 9.87 \quad 9.73$

$9.93 \quad 9.66 \quad 9.66$

$9.44 \quad 9.52$

$9.49 \quad 9.51 \quad-$

$$
9.54 \quad 9.51
$$$$
19.0 \quad 18.4 \quad 18.1
$$$$
18.8 \quad 18.5 \quad 18.5
$$$$
\begin{array}{ll}
18.38 & 18.69 \\
18.44
\end{array}
$$$$
\begin{array}{ll}
18.38 & 18.44 \\
18.65 & 18.67 \\
18.35 & 18.60
\end{array}
$$$$
\begin{array}{ll}
18.35 & 18.60 \\
18.34 & 18.34 \\
18.65 & 18.7
\end{array}
$$$$
\begin{array}{llc}
18.65 & 18.7 & - \\
18.68 & 18.75 & 18.4
\end{array}
$$

$18.35 \quad .00 \quad .12 \quad 1.00 \mathrm{NS}$

$17.95 \quad .06 \quad .14 \quad 1.68 \mathrm{NS}$

$\begin{array}{llll}18.98 & .14 & .07 & 7.02 \mathrm{NS}\end{array}$

17.60 Neg. .24 .62NS

18.85 Neg. $.15 \quad .36 \mathrm{NS}$

$\begin{array}{llll}18.30 \quad .24 & .20 \quad 3.98 \mathrm{NS}\end{array}$

$\begin{array}{llll}8.93 & .069 & .215 & 1.18 \mathrm{NS}\end{array}$

9.74 Neg. $.16 \quad .14 \mathrm{NS}$

$9.50 \quad$ Neg. $\quad .04 \quad .64 \mathrm{NS}$

\begin{tabular}{ccccccccc} 
/ ICPS & 9.19 & 8.93 & 9.16 & 9.09 & - & - & - \\
/XRF & 9.47 & 9.53 & 9.47 & 9.49 & - & - & - \\
\hline \multicolumn{7}{c}{$\mathrm{Fe}_{2} \mathrm{O}_{3}$} \\
\hline GSF/Chem. & 1.70 & 1.80 & 1.80 & 1.78 & .038 & .025 & 5.70 NS \\
& 1.76 & 1.80 & 1.81 & & & &
\end{tabular}

$\begin{array}{llll}2.1 & 1.7 & 1.9 \\ & 1.9 & 1.7 & 2.1\end{array}$

GSC/F* $\quad 1.76 \quad 1.63 \quad 1.72$

$\begin{array}{rrrr} & 1.84 & 1.72 & 1.62 \\ \text { NIM } & .56 & .60 & .63\end{array}$

$\begin{array}{llll} & .61 & .74 & .60\end{array}$

$\begin{array}{lllllll}1.8 & 1.7 & 1.9 & 1.92 & \text { Neg. } & .18 & .84 \mathrm{NS}\end{array}$

$\begin{array}{llll}\text { ETH/AAS } & 1.9 & 2.0 & 2.2\end{array}$

\begin{tabular}{|c|c|c|c|c|c|c|c|c|c|c|c|c|c|c|c|}
\hline *KJell/XRF & 21.6 & 21.4 & 21.5 & 21.5 & - & - & - & ETH & .99 & 1.18 & 1.06 & 1.08 & - & - & - \\
\hline & & & $\mathrm{Al}_{2} \mathrm{O}_{3}$ & & & & & & & & Feo & & & & \\
\hline \multirow[t]{2}{*}{ GSF/Chemb } & 18.54 & 18.54 & 18.45 & 18.54 & Neg. & .05 & $.12 \mathrm{NS}$ & GSF/Chem & $7 \cdot 35$ & $7 \cdot 33$ & $7 \cdot 32$ & $7 \cdot 33$ & .008 & .008 & $3.00 \mathrm{NS}$ \\
\hline & 18.54 & 18.57 & 18.59 & & & & & & $7 \cdot 33$ & $7 \cdot 33$ & 7.32 & & & & \\
\hline \multirow[t]{2}{*}{ *BMNH/Chem. } & 18.97 & 18.97 & $19 \cdot 30$ & 19.03 & - & - & - & *BMNH/Chem & $7 \cdot 36$ & $7 \cdot 47$ & 7.43 & $7 \cdot 37$ & - & - & - \\
\hline & 18.92 & 18.98 & 19.05 & & & & & & $7 \cdot 34$ & $7 \cdot 32$ & 7.32 & & & & \\
\hline \multirow[t]{2}{*}{ *BMNH/XRF } & 18.4 & 18.4 & $18 \cdot 3$ & 18.37 & .06 & 0 & * & $\mathrm{GSC} / \mathrm{B}$ & 7.2 & $7 \cdot 4$ & $7 \cdot 3$ & $7 \cdot 3$ & .08 & .06 & $4.50 \mathrm{NS}$ \\
\hline & 18.4 & 18.4 & 18.3 & & & & & & $7 \cdot 3$ & 7.4 & 7.2 & & & & \\
\hline \multirow[t]{2}{*}{ *BMNH/XRF } & 18.64 & 18.75 & 18.83 & 18.72 & Neg. & .12 & $.34 \mathrm{NS}$ & GSC/F* & 7.25 & $7 \cdot 34$ & 7.24 & 7.28 & .02 & .05 & $1.46 \mathrm{NS}$ \\
\hline & 18.73 & 18.81 & 18.56 & & & & & & 7.21 & 7.29 & 7.35 & & & & \\
\hline \multirow[t]{2}{*}{$\mathrm{GSC} / \mathrm{A}^{*}$} & 18.0 & 18.2 & 18.0 & 18.1 & Neg. & .25 & $.34 \mathrm{NS}$ & NIM/Vanad & 8.36 & 8.42 & 8.34 & 8.39 & .02 & .04 & $1.54 \mathrm{NS}$ \\
\hline & 18.5 & 17.9 & 18.2 & & & & & & 8.45 & 8.42 & 8.37 & & & & \\
\hline \multirow[t]{2}{*}{$\mathrm{GSC} / \mathrm{E}$} & 18.65 & 18.55 & 18.65 & 18.66 & Neg. & .06 & .9 INS & USGSR/Chem & $7 \cdot 2$ & $7 \cdot 3$ & 7.2 & 7.20 & 0 & .08 & $*$ \\
\hline & 18.70 & 18.67 & 18.72 & & & & & & 7.2 & 7.1 & 7.2 & & & & \\
\hline
\end{tabular}




\begin{tabular}{|c|c|c|c|c|c|c|c|c|c|c|c|c|c|c|c|}
\hline \multirow[b]{3}{*}{ Org./Meth. } & \multirow{3}{*}{ Botcle } & \multirow{2}{*}{\multicolumn{2}{|c|}{ Number }} & \multirow[b]{3}{*}{ Mean } & \multicolumn{2}{|c|}{ Standard Deviation } & \multirow{3}{*}{$\begin{array}{c}F \\
\text { ratio } \\
\end{array}$} & & Bottle & & mber & & Botcle - & Exror & $F$ \\
\hline & & & & & Botrle & Error & & Orea/Methe & 1 & $\underline{\underline{2}}$ & 3 & Mean & $2 \mathrm{df}$ & $3 \mathrm{df}$ & ratio \\
\hline & & 2 & 3 & & $2 \mathrm{df}$ & $3 \mathrm{df}$ & & & & & $\mathrm{CaO}(\mathrm{c}$ & t. ) & & & \\
\hline & & & $\mathrm{FeO}($ & nt.) & & & & $\mathrm{GSC} / \mathrm{G}^{*}$ & 10.97 & 11.14 & 11.24 & 11.24 & Neg. & 0.29 & 0.11 NS \\
\hline Liege/Chem & 6.24 & 6.93 & - & 6.81 & Neg. & 0.08 & $0.55 \mathrm{NS}$ & & 11.65 & 11.33 & 11.10 & & & & \\
\hline & 6.81 & 6.84 & - & & & 0.08 & 0.050 & BIO/AAS & 11.38 & 11.35 & 11.75 & 11.40 & .12 & .16 & $2.18 \times S$ \\
\hline & 6.71 & 6.74 & & & & & & & 11.14 & 11.35 & 11.43 & & & & \\
\hline ETH/Chen & $7 \cdot 78$ & 7.85 & - & 7.72 & - & & & *NDM/XRF & 11.49 & 11.49 & 11.06 & 11.56 & Neg. & .19 & $.04 \mathrm{NS}$ \\
\hline ETH & 8.20 & 8.03 & 8.05 & 8.09 & - & 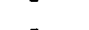 & 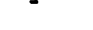 & & 11.49 & 11.71 & 11.71 & & & & \\
\hline & & & $\mathrm{MgO}$ & & & $=$ & - & & 11.60 & 11.71 & 11.60 & & & & \\
\hline & & & & & & & & & 11.71 & 11.49 & 11.60 & & & & \\
\hline GSF/.Chem & 10.32 & 10.31 & 10.32 & 10.32 & 0 & .007 & * & NIM/ICPS & 11.80 & 11.70 & 11.70 & 11.73 & Neg. & .06 & $.50 \mathrm{NS}$ \\
\hline & 10.31 & 10.32 & 10.31 & & & & & & 11.70 & 11.70 & 11.80 & & & & \\
\hline *BMNH/Chem & 10.08 & 10.07 & 10.18 & 10.13 & - & - & - & USGSR/Chem & 11.1 & 10.9 & 11.2 & 11.07 & .14 & .10 & $5.17 \mathrm{NS}$ \\
\hline & 10.23 & 10.08 & 10.4 & & & & & & 10.9 & 11.0 & 11.3 & & & & \\
\hline *BMNH/XRF & 10.20 & 10.17 & 10.19 & 10.10 & Neg. & .13 & $.16 \mathrm{NS}$ & WHOI/XRF & 11.18 & 11.15 & 11.07 & 11.11 & .05 & .04 & $3.34 \mathrm{NS}$ \\
\hline & 10.03 & 10.10 & 9.94 & & & & & & 11.09 & 11.16 & 11.01 & & & & \\
\hline *BMNH/XRF & 10.19 & 10.24 & 10.27 & 10.22 & .02 & .04 & $1.58 \mathrm{NS}$ & UWUr 2/XRF & 11.10 & 11.15 & 10.95 & 11.06 & .06 & $.0_{4}$ & $5.00 \mathrm{NS}$ \\
\hline & 10.20 & 10.15 & 10.24 & & & & & & 11.10 & 11.05 & 11.00 & & & & \\
\hline $\mathrm{GSC} / \mathrm{A}$ & 10.2 & 10.0 & 10.3 & 10.15 & .12 & .09 & $4.20 \mathrm{NS}$ & WSU/XRF & 10.61 & 10.75 & 10.69 & 10.66 & .004 & .057 & $1.01 \mathrm{NS}$ \\
\hline & 10.0 & 10.1 & 10.3 & & & & & & 10.62 & 10.61 & 10.69 & & & & \\
\hline $\mathrm{GSC} / \mathrm{G}$ & 10.10 & 10.16 & 10.21 & 10.14 & Neg. & .07 & $.33 \mathrm{NS}$ & USGSR/XRF & 11.60 & 11.50 & 11.39 & 11.54 & .14 & .12 & $3.64 \mathrm{NS}$ \\
\hline & 10.20 & 10.17 & 10.13 & & & & & & 11.68 & 11.79 & 11.32 & & & & \\
\hline BIO/AAS & $9 \cdot 90$ & 9.86 & 10.34 & 9.96 & .11 & .16 & $2 . \mathrm{O}_{4} \mathrm{NS}$ & *Toron/INAA & 8.08 & 8.13 & 6.87 & 7.80 & Neg. & .58 & $.37 \mathrm{NS}$ \\
\hline & 9.83 & $9 \cdot 90$ & $9 \cdot 96$ & & & & & & 8.29 & $7 \cdot 39$ & 8.53 & & & & \\
\hline *NDM/XRF & 9.66 & 10.00 & 9.84 & 10.02 & Neg. & .18 & $.25 \mathrm{NS}$ & & $7 \cdot 59$ & 7.98 & $7 \cdot 34$ & & & & \\
\hline & $9 \cdot 94$ & 10.05 & 10.15 & & & & & *HMI/INAA-W & $7 \cdot 80$ & 7.25 & - & 7.45 & Neg. & .24 & $.50 \mathrm{NS}$ \\
\hline & 10.28 & 10.18 & 10.02 & & & & & & $7 \cdot 35$ & 7.65 & - & & & & \\
\hline & 10.17 & 10.04 & 10.02 & & & & & & 7.41 & 7.25 & - & & & & \\
\hline $\mathrm{NIM} / \mathrm{ICPS}$ & 9.71 & $9 \cdot 95$ & 9.93 & $9 \cdot 91$ & .01 & .12 & $1.02 \mathrm{NS}$ & $*$ HMI/INAA-B & $5 \cdot 32$ & 5.02 & - & 5.09 & Neg. & .17 & $.12 \mathrm{NS}$ \\
\hline & 9.95 & $9 \cdot 86$ & 10.07 & & & & & & 5.06 & 5.25 & - & & & & \\
\hline USGSR/Chem & 10.1 & 10.1 & 10.2 & 10.15 & 0 & .07 & * & & 4.97 & 4.93 & - & & & & \\
\hline & 10.2 & 10.2 & 10.1 & & & & & *LASL/INAA-I & 9.2 & 8.0 & - & 8.55 & Neg. & .46 & $.64 \mathrm{NS}$ \\
\hline WHOI/XRF & 10.71 & 10.64 & 10.59 & 10.62 & .07 & .04 & $5.70 \mathrm{NS}$ & & 3.5 & 8.9 & - & & & & \\
\hline & 10.67 & 10.62 & 10.49 & & & & & & 8.5 & 9.2 & - & & & & \\
\hline UWUrz/XRF & 9.9 & 10.1 & 9.9 & $9 \cdot 95$ & .08 &.$\alpha_{4}$ & $9.00 \mathrm{NS}$ & Tohok/IPAA & 11.43 & 11.52 & 11.89 & 11.62 & .06 & .28 & 1. IONS \\
\hline & $9 \cdot 9$ & 10.0 & $9 \cdot 9$ & & & & & & 11.35 & 12.05 & 11.47 & & & & \\
\hline WSU/XRF & 8.49 & 8.91 & 8.88 & 8.80 & .12 & .12 & $2.78 \mathrm{NS}$ & UInd/ICPS & 11.1 & 11.1 & 11.0 & 11.08 & Neg. & .12 & $.78 \mathrm{NS}$ \\
\hline USGSR/XRF & 8.77 & 8.81 & 8.91 & & & & & Exxon/DCPAS & 10.9 & 11.2 & 11.2 & & & & \\
\hline & 10.10 & 10.14 & 10.12 & 10.14 & .03 & .03 & $2.88 \mathrm{NS}$ & & 10.85 & 10.89 & - & 10.82 & Neg. & .08 & $.01 N s$ \\
\hline & 10.10 & 10.22 & 10.14 & & & & & & $\begin{array}{l}10.90 \\
10.72\end{array}$ & $\begin{array}{l}10.79 \\
10.77\end{array}$ & - & & & & \\
\hline *Toron/IRAA & $5 \cdot 34$ & 5.93 & 6.44 & 5.65 & .27 & .38 & $2.54 \mathrm{NS}$ & CRPG/MWPS & 10.94 & 11.13 & - & 11.10 & .05 & .10 & $1.49 \mathrm{NS}$ \\
\hline & 5.55 & 5.77 & $5 \cdot 97$ & & & & & & 11.13 & 11.18 & - & & & & \\
\hline & 4.90 & 5.60 & 5.37 & & & & & ETH/AAS & 11.15 & 11.20 & - & 11.18 & - & - & - \\
\hline Tohok/IPAA & 10.43 & 10.21 & 10.20 & 10.27 & Neg. & .11 & $\cdot 57 \mathrm{NS}$ & $\mathrm{ETH} / \mathrm{XRF}$ & 11.47 & 11.48 & 11.38 & 11.44 & - & - & - \\
\hline & 10.20 & 10.36 & 10.20 & & & & & *Kje11/AAS & 8.25 & 8.25 & 8.25 & 8.25 & - & - & - \\
\hline UInd/ICPS & 9.65 & 9.42 & $9 \cdot 36$ & $9 \cdot 48$ & .058 & .083 & $1.99 \mathrm{NS}$ & $/$ ICPS & 8.56 & 7.52 & 7.66 & 7.91 & - & - & - \\
\hline & $9 \cdot 50$ & $9 \cdot 50$ & 9.47 & & & & & /XRF & 8.15 & 8.11 & 8.11 & 8.12 & - & - & - \\
\hline Exxon/DCPAS & $\begin{array}{l}10.25 \\
10.25\end{array}$ & $\begin{array}{l}10.32 \\
10.13\end{array}$ & - & 10.22 & Neg. & .08 & $.23 \mathrm{NS}$ & & & & $\mathrm{Na}_{2} \mathrm{O}$ & & & & \\
\hline & 10.10 & 10.23 & - & & & & & GSF/Chem & 1.91 & 1.94 & 1.94 & 1.92 & .012 & .009 & $4.25 \mathrm{NS}$ \\
\hline GRPG/MWPS & 9.80 & 9.85 & - & 9.84 & .07 &.$\alpha_{4}$ & $6.05 \mathrm{NS}$ & & 1.91 & 1.93 & 1.92 & & & & \\
\hline & 9.76 & 9.93 & - & & & & & $\mathrm{GSC} / \mathrm{A}$ & 1.7 & 1.5 & 1.5 & 1.58 & .11 & .09 & $3.50 \mathrm{NS}$ \\
\hline *USGSR/AAS & 5.69 & 5.93 & 5.53 & 5.70 & .23 & .097 & 12.30 & & 1.7 & 1.7 & 1.4 & & & & \\
\hline & 5.84 & 5.89 & 5.35 & & & & & GSC/G* & 1.90 & 1.88 & 1.88 & 1.92 & Neg. & .05 & $.79 \mathrm{NS}$ \\
\hline ETH/AAS & 9.93 & 9.81 & - & 9.87 & - & - & - & & 2.01 & 1.92 & 1.92 & & & & \\
\hline ETH/XRF & 9.94 & 9.89 & 9.75 & 9.86 & - & - & - & BIO/AAS & 1.87 & 1.86 & 1.91 & 1.87 & .03 & .02 & $4.50 \mathrm{NS}$ \\
\hline *KjelI/AAS & 6.40 & 6.20 & 6.40 & 6.33 & - & - & - & & 1.82 & 1.86 & 1.90 & & & & \\
\hline /ICPS & 7.1 & $7 \cdot 1$ & - & 7.1 & - & - & - & NDM/AAS & 1.90 & 1.94 & 1.91 & 1.94 & Neg. & .05 & $.25 \mathrm{NS}$ \\
\hline$/ \mathrm{XRF}$ & 6.1 & 6.1 & 6.3 & 6.2 & - & - & - & & 2.02 & 1.93 & 1.94 & & & & \\
\hline & & & $\mathrm{CaO}$ & & & & & USGSR/Chem & 1.9 & 1.9 & 1.9 & 1.92 & $<.000$ & $.0_{4}$ & $1.00 \mathrm{NS}$ \\
\hline & & & & & & & & & 2.0 & 1.9 & 1.9 & & & & \\
\hline GSF/Chem & 11.22 & 11.22 & 11.18 & 11.20 & 0 & .028 & * & Parma/Fiph & 1.88 & 1.93 & - & 1.90 & .018 & .012 & 8.00 \\
\hline & 11.18 & 11.18 & 11.22 & & & & & & 1.89 & 1.90 & - & & & & \\
\hline *BMNH/Chem & 11.50 & 11.49 & 11.38 & 11.43 & - & - & - & & 1.89 & 1.91 & - & & & & \\
\hline & 11.30 & 11.53 & 11.37 & & & & & WHOI/XRF & 1.61 & 1.58 & 1.62 & 1.61 & Neg. & .04 & .3ONS \\
\hline *BMNH/XRF & 11.52 & 11.50 & 11.46 & 11.47 & Neg. & .04 & .5 ONS & & 1.57 & 1.66 & 1.59 & & & & \\
\hline & 11.45 & 11.45 & 11.44 & & & & & UWUrz/XRF & 2.0 & 1.8 & 1.9 & 1.95 & .09 & .07 & $4.00 \mathrm{Ns}$ \\
\hline *BMNH/XRF & 11.48 & 11.49 & 11.37 & 11.38 &.$\alpha_{4}$ & .09 & $1.46 \mathrm{WS}$ & & 2.1 & 1.9 & 2.0 & & & & \\
\hline & 11.35 & 11.39 & 11.22 & & & & & WSU/XRF & 2.67 & 2.85 & 2.97 & 2.86 & Neg. & .12 & $.46 \mathrm{NS}$ \\
\hline GSC/A & 11.7 & 11.5 & 11.6 & 11.6 & .08 & .06 & $4 \cdot 50 \mathrm{NS}$ & & 2.92 & 2.93 & 2.83 & & & & \\
\hline & 11.6 & 11.5 & 11.7 & & & & & & & & & & & & \\
\hline
\end{tabular}


Tab1e 2. Analytical data for USGS-DNC-1 (cont.)

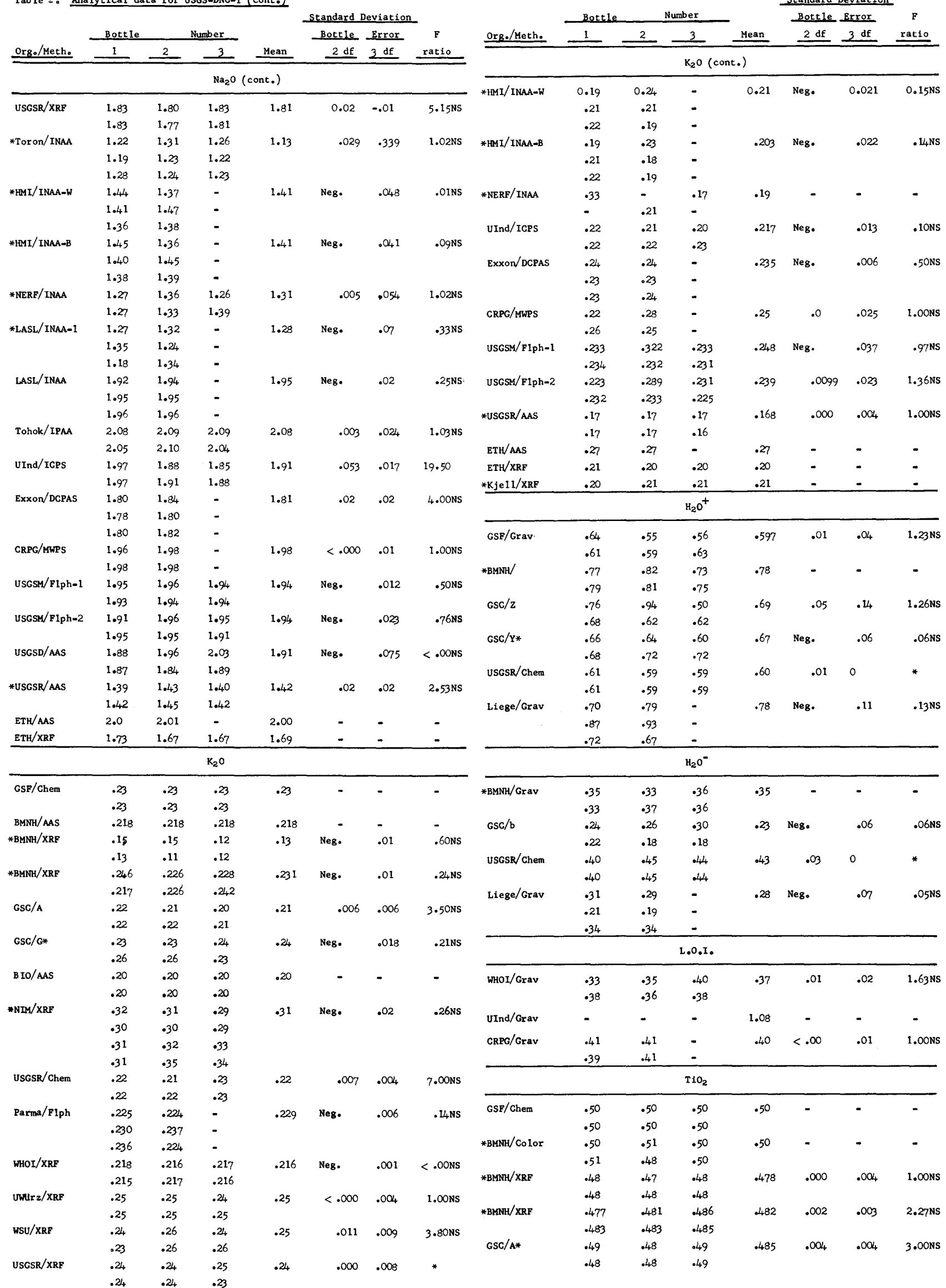


Table 2. Analytical data for USGS-DNC-1 (cont.)

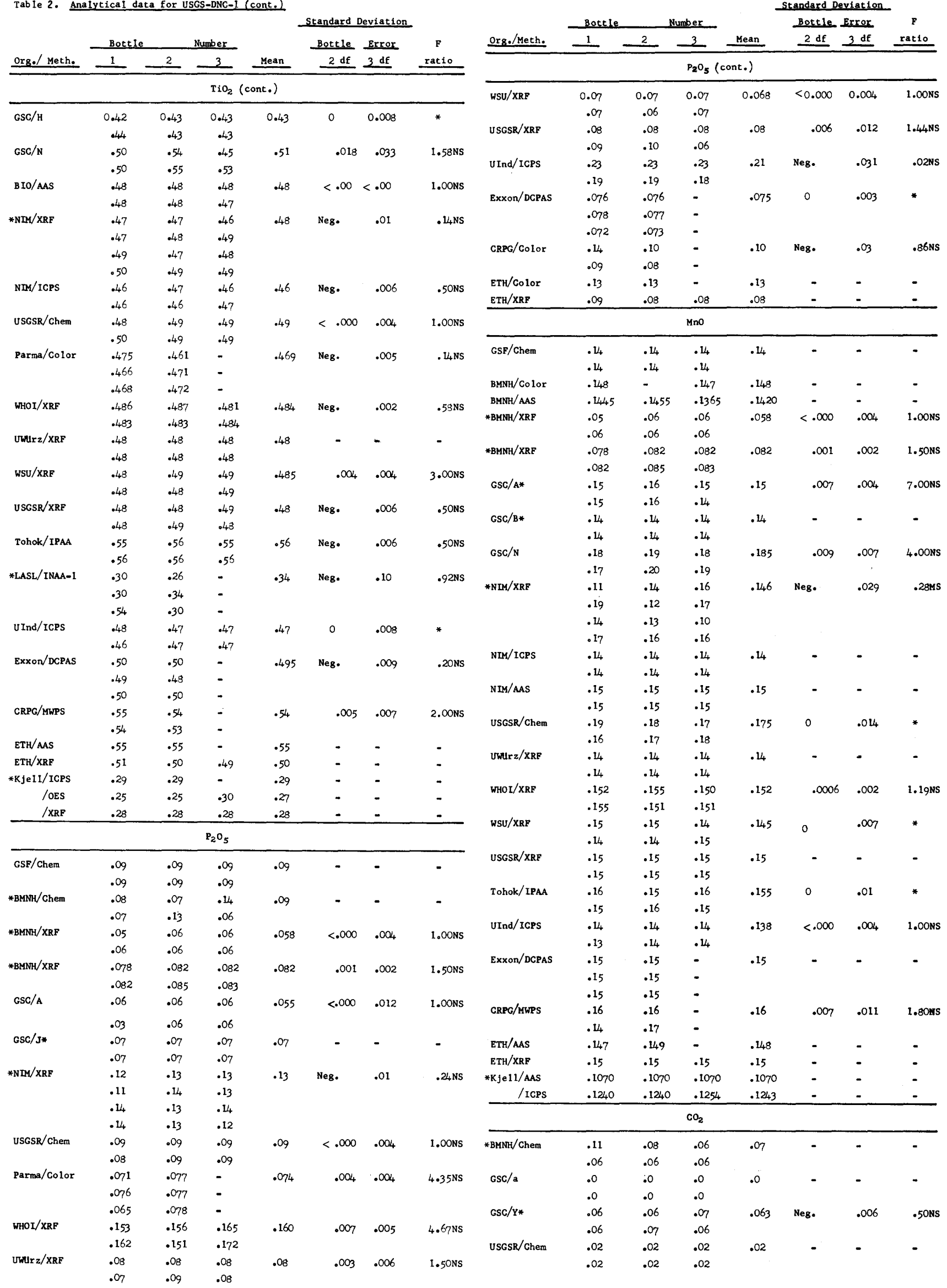




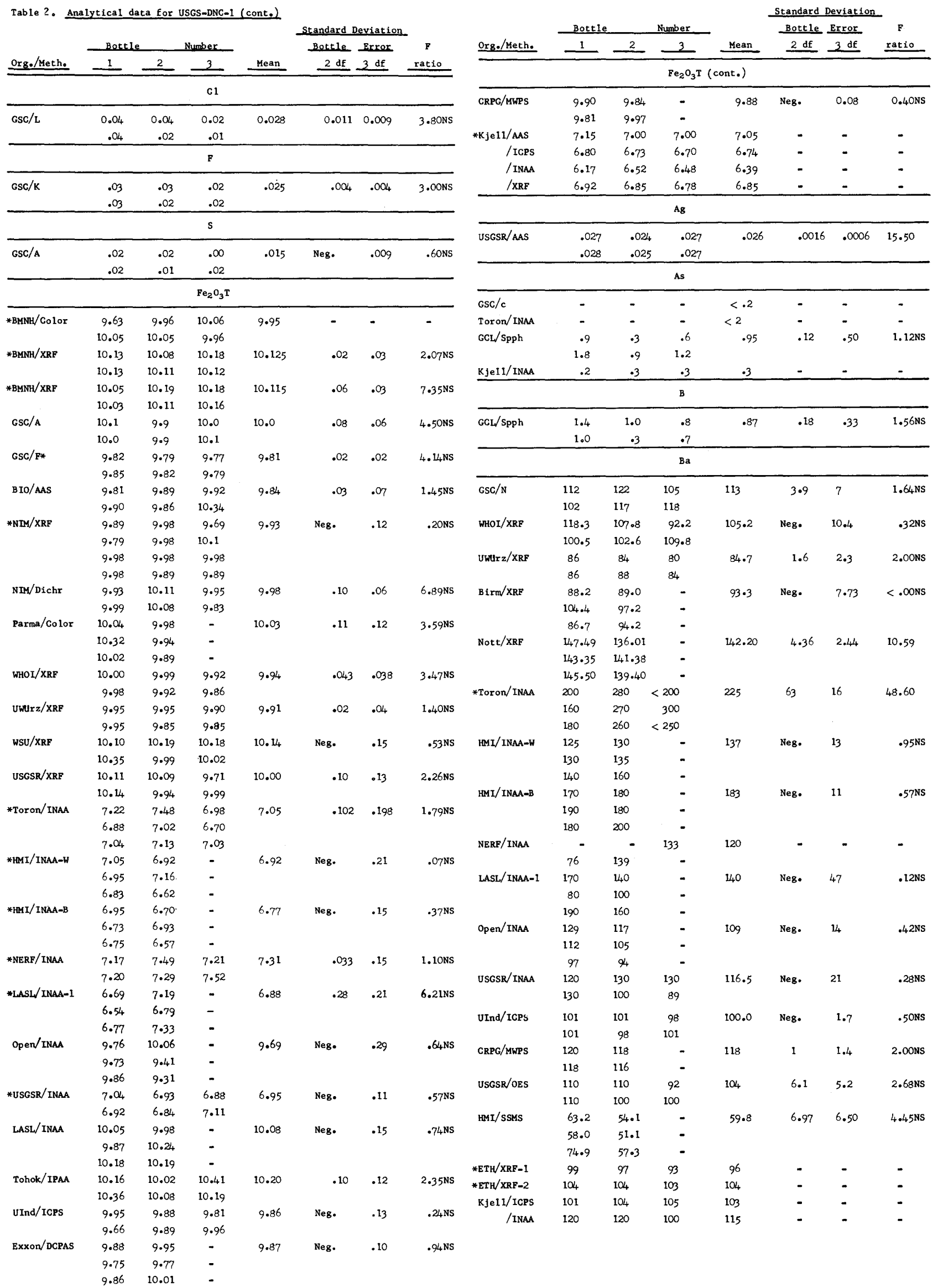


Table 2. Analytical data for USGS-DNC-1 (cont.)

\begin{tabular}{|c|c|c|c|c|c|c|c|c|c|c|c|c|c|c|c|}
\hline \multirow{4}{*}{ Org./Meth. } & \multirow{4}{*}{$\begin{array}{l}\text { Bottie } \\
1 \\
\end{array}$} & \multirow{3}{*}{\multicolumn{2}{|c|}{ Number }} & \multirow{4}{*}{ Mean } & & & & & & & & & & & \\
\hline & & & & & Itandard De & viation & & & Bott & & Number & & Bottile & Error & $\mathbf{P}$ \\
\hline & & & & & Bottle & Error & F & Org./Meth. & 1 & 2 & 3 & Kean & $2 \mathrm{df}$ & $3 \mathrm{df}$ & ratio \\
\hline & & 2 & 3 & & $\underline{2 d f}$ & $3 \mathrm{df}$ & ratio & & & & Co (c & & & & \\
\hline & & & $\mathrm{Be}$ & & & & & NIM/AAS & 56 & 54 & 56 & $55 \cdot 3$ & Neg. & 1.2 & $0.50 \mathrm{NS}$ \\
\hline BIO/AAS & 0.8 & 0.7 & 0.7 & 0.73 & Neg. & 0.06 & $0.50 \mathrm{Ns}$ & & 54 & 56 & 56 & & & & \\
\hline & .7 & .8 & .7 & & & & & $\mathrm{NIM} / \mathrm{INAA}$ & 58 & 58 & 60 & 58.8 & 1.0 & .4 & 13.00 \\
\hline GCL/AAS & .6 & .8 & .8 & .80 & Neg. & .20 & $.50 N S$ & & 59 & 58 & 60 & & & & \\
\hline & 1.0 & 1.0 & .6 & & & & & Parma/AAS & 63 & 62 & - & 62.2 & Neg. & 1.29 & . IONS \\
\hline & & & Gd & & & & & & 63 & 63 & - & & & & \\
\hline & & & & & & & & & $\begin{array}{l}60 \\
57.5\end{array}$ & $\begin{array}{l}62 \\
58.6\end{array}$ & 57.5 & & & & \\
\hline *BIO/AAS & $\begin{array}{l}79 \\
89\end{array}$ & $\begin{array}{l}85 \\
87\end{array}$ & $\begin{array}{l}85 \\
93\end{array}$ & 86.3 & Neg. & $5 \cdot 3$ & $.45 \mathrm{NS}$ & WHOL/XRF & $\begin{array}{l}57 \cdot 5 \\
57 \cdot 8\end{array}$ & $\begin{array}{l}58.6 \\
58.0\end{array}$ & $\begin{array}{l}57.5 \\
56.9\end{array}$ & $57 \cdot 7$ & .5 & .4 & $4.53 \mathrm{NS}$ \\
\hline *WAIT/IDMS & 89 & 86 & - & 89.8 & 2.9 & 3.6 & $2.28 \mathrm{NS}$ & UWUrz/XRF & 53 & 56 & 52 & 54.7 & 2.8 & 1.8 & 5.60NS \\
\hline & 96 & 88 & - & & & & & & 55 & 60 & 52 & & & & \\
\hline GCL/AAS & .20 & .20 & .42 & .27 & .12 & .02 & 50.33 & Llege/XRP & 50 & 46 & - & 49.2 & 1.6 & 2.4 & $2.3 \mathrm{INS}$ \\
\hline & .24 & .16 & .40 & & & & & & 48 & 49 & - & & & & \\
\hline & & & $\mathrm{Ce}$ & & & & & & 54 & 48 & - & & & & \\
\hline & & & ve & & & & & Nott $/ \mathbf{X R F}$ & 51.32 & 48.36 & - & 52.32 & 1.22 & 2.68 & $1.63 \mathrm{NS}$ \\
\hline $\mathrm{Birm} / \mathrm{XRF}$ & 9.7 & 10.9 & - & 10.6 & .56 & .42 & $6.38 \mathrm{NS}$ & & 52.98 & 53.38 & - & & & & \\
\hline & 10.8 & 11.1 & - & & & & & & 56.85 & $51.0_{4}$ & - & & & & \\
\hline & 9.9 & 11.0 & - & & & & & Toron/INAA & 65.6 & 68.1 & 61.7 & 66.1 & 2.4 & 3.0 & $3.05 \mathrm{NS}$ \\
\hline Nott/XRP & 15.26 & $17 \cdot 10$ & - & 13.22 & Neg. & 3.51 & $<$. OONS & & 68.2 & 65.3 & 61.4 & & & & \\
\hline & 13.37 & 8.26 & - & & & & & & 73.4 & 64.7 & 66.2 & & & & \\
\hline & 11.12 & 14.20 & - & & & & & HMI/INAA-W & 60.7 & 59.4 & - & 59.3 & Neg. & 1.8 & $.13 \mathrm{NS}$ \\
\hline Toron/INAA & 9.4 & 10.1 & 11.2 & 10.92 & Neg. & 1.0 & $.23 \mathrm{NS}$ & & 59.7 & 61.1 & - & & & & \\
\hline & 11.8 & 12.7 & 11.0 & & & & & & 58.3 & 56.6 & - & & & & \\
\hline & 10.6 & 10.7 & 10.8 & & & & & HMI/INAA-B & 58.0 & 56.7 & - & 57.1 & Neg. & 1.0 & $.48 \mathrm{NS}$ \\
\hline HMI/INAA-W & 8.82 & 8.53 & - & 8.45 & .32 & .49 & $2.29 \mathrm{NS}$ & & 56.9 & 58.2 & - & & & & \\
\hline & 9.07 & 8.45 & - & & & & & & $57 \cdot 3$ & 55.6 & - & & & & \\
\hline & 8.37 & 7.45 & - & & & & & NERF/INAA & 57.2 & 58.7 & 57.6 & $57 \cdot 8$ & Neg. & .77 & $.75 \mathrm{NS}$ \\
\hline HMI/INAA-B & 9.05 & 8.57 & - & 8.43 & .60 & .42 & $7.02 \mathrm{NS}$ & & $57 \cdot 2$ & 57.1 & 58.6 & & & & \\
\hline & 9.06 & $7 \cdot 75$ & - & & & & & LASL/INAA-1 & 54 & 55 & - & 55.7 & 2.2 & 1.5 & $7.69 \mathrm{NS}$ \\
\hline & 8.55 & 7.61 & - & & & & & & 54 & 59 & - & & & & \\
\hline *NERF/INAA & - & 2.5 & 3.6 & 2.6 & Neg. & .76 & $.35 \mathrm{NS}$ & & 54 & 58 & - & & & & \\
\hline & - & 2.3 & 2.1 & & & & & Open/INAA & $57 \cdot 8$ & 56.4 & - & 57.23 & .28 & .50 & I.95NS \\
\hline LASL/INAA-1 & 13 & 13 & - & 13.2 & 1.1 & .8 & $6.25 \mathrm{NS}$ & & $57 \cdot 3$ & $57 \cdot 7$ & - & & & & \\
\hline & 12 & 15 & - & & & & & & $57 \cdot 6$ & 56.9 & - & & & & \\
\hline & 12 & $u_{4}$ & - & & & & & USGSR/INAA & $57 \cdot 2$ & $55 \cdot 7$ & 55.0 & 55.6 & Neg. & 1.12 & $.95 \mathrm{NS}$ \\
\hline Open/INAA & 8.4 & 7.7 & - & 8.33 & .54 & .30 & 12.26 & & 55.5 & 53.9 & 56.2 & & & & \\
\hline & 9.0 & $7 \cdot 9$ & - & & & & & $\mathrm{LASL} / \mathrm{INAA}$ & 58.1 & 56.9 & - & 57.3 & Neg. & $\cdot 70$ & $.03 \mathrm{NS}$ \\
\hline & 8.8 & 8.2 & - & & & & & & 56.3 & 57.4 & - & & & & \\
\hline USGSR/INAA & 9 & 10 & 9 & 9.2 & .71 & .41 & $7.00 \mathrm{NS}$ & & $57 \cdot 3$ & $57 \cdot 7$ & - & & & & \\
\hline & 9 & 10 & $B$ & & & & & Tohok/IPAA & 62 & 63 & 62 & 62.3 & .3 & 1 & $1.17 \mathrm{NS}$ \\
\hline Tohok/IPAA & $9 \cdot 5$ & 8.3 & 8.5 & 8.58 & Neg. & .5 & $.67 \mathrm{NS}$ & & 61 & 62 & 64 & & & & \\
\hline & 8.3 & 8.4 & 8.5 & & & & & UInd/AAS & 57 & 52 & 56. & 54.3 & Neg. & 3.9 & $.68 \mathrm{NS}$ \\
\hline Che1s/INAA & 8.9 & 9.0 & - & 8.50 & Neg. & .96 & $.46 \mathrm{NS}$ & & 56 & 57 & 48 & & & & \\
\hline & 6.7 & 8.8 & - & & & & & Exxon/DCPAS & 55.9 & 53.4 & - & 54.8 & 2.3 & 1.4 & 9.16 \\
\hline & 9.1 & 8.5 & - & & & & & & 58.6 & 53.1 & - & & & & \\
\hline Genev/INAA & 11.94 & 13.03 & 13.02 & 12.30 & .37 & .53 & $1.96 \mathrm{NS}$ & & 54.9 & 52.7 & - & & & & \\
\hline & I1.43 & 12.12 & 12.23 & & & & & CRPG/MWPS & 72 & 76 & - & 76 & .5 & 2.9 & $1.06 \mathrm{NS}$ \\
\hline & 12.15 & 12.54 & 11.03 & & & & & & 77 & 79 & - & & & & \\
\hline $\mathrm{HMI} / \mathrm{SSMS}$ & 19.2 & 13.4 & - & $1_{4} .0$ & 3.86 & 3.07 & $5.74 \mathrm{NS}$ & USGSR/OES & 45 & 44 & 47 & 47.5 & Neg. & 3.3 & $.05 \mathrm{NS}$ \\
\hline & 13.7 & 12.2 & - & & & & & & 50 & 50 & 49 & & & & \\
\hline & 18.0 & $7 \cdot 3$ & - & & & & & HMI/SSMS & 20.2 & 26.9 & - & 19.2 & Neg. & 6.6 & $.03 \mathrm{NS}$ \\
\hline $\mathrm{ETH} / \mathrm{XRP}$ & - & - & 9 & - & - & - & - & & 25.2 & 13.2 & - & & & & \\
\hline K Jel 1/IMAa & 8.5 & 8.4 & 8.3 & 8.4 & - & - & - & & 13.5 & 16.1 & - & & & & \\
\hline & & & GI & & & & & $\mathrm{ETH} / \mathrm{XRF}$ & 52 & 53 & 55 & 53 & - & - & - \\
\hline & & & & & & & & $\mathrm{KJel} \mathrm{L} / \mathrm{AAS}$ & 78 & 82 & 78 & 79 & - & - & - \\
\hline HMI/SSMS & 28.4 & 43.4 & - & 31.2 & Neg. & 8.4 & $.06 \mathrm{NS}$ & $/$ ICPS & 70 & 70 & 84 & 75 & - & - & - \\
\hline & $37 \cdot 8$ & $27 \cdot 3$ & - & & & & & / INAA & 52.5 & 55.3 & 54.5 & 54.1 & - & - & $=$ \\
\hline & 24.9 & 25.3 & - & & & & & & & & $\mathrm{cr}$ & & & & \\
\hline Munich & 105 & 99 & - & 95.5 & 5.2 & 6.4 & $2.96 \mathrm{NS}$ & & & & & & & & \\
\hline & 100 & 84 & - & & & & & GSF/OBS & 390 & 450 & 360 & 355 & Neg. & 79 & $.03 N S$ \\
\hline & 95 & 90 & - & & & & & & 300 & 280 & 350 & & & & \\
\hline & & & Co & & & & & BMNH/XRF & 253 & 260 & 249 & 252.3 & 1.8 & 4.1 & $1.36 \mathrm{NS}$ \\
\hline & & & & & & & & & 254 & 250 & 248 & & & & \\
\hline GSF/OES & 54 & 44 & 52 & 50.3 & 3.4 & 2.4 & $4.80 \mathrm{NS}$ & $\mathrm{GSC} / \mathrm{N}$ & 270 & 290 & 260 & 278 & Neg. & 17 & $.4 \mathrm{INS}$ \\
\hline & 50 & 48 & 54 & & & & & & 270 & 280 & 300 & & & & \\
\hline $\mathrm{GSC} / \mathrm{N}$ & 62 & 70 & 64 & 64.5 & 3.8 & 1.8 & 10.10 & BIO/AAS & 343 & 342 & 336 & 339 & 2.2 & 10 & $1.09 \mathrm{NS}$ \\
\hline & 59 & 67 & 65 & & & & & & $32 \mathrm{I}$ & 352 & 342 & & & & \\
\hline BIO/AAS & 55 & 56 & 57 & 55 & 1.0 & 1.0 & $3.17 \mathrm{NS}$ & NDM/ICPS & 300 & 330 & 315 & 311 & Neg. & 14 & $.14 \mathrm{NS}$ \\
\hline & 53 & 55 & 56 & & & & & & 315 & 300 & 305 & & & & \\
\hline & & & & & & & & $\mathrm{NIM} / \mathrm{INAA}$ & 313 & 302 & 303 & 307 & Neg. & 7.0 & $.61 \mathrm{NS}$ \\
\hline & & & & & & & & & 303 & 303 & 317 & & & & \\
\hline
\end{tabular}


Table 2. Analytical data for USGS-DNG-1 (cont.)

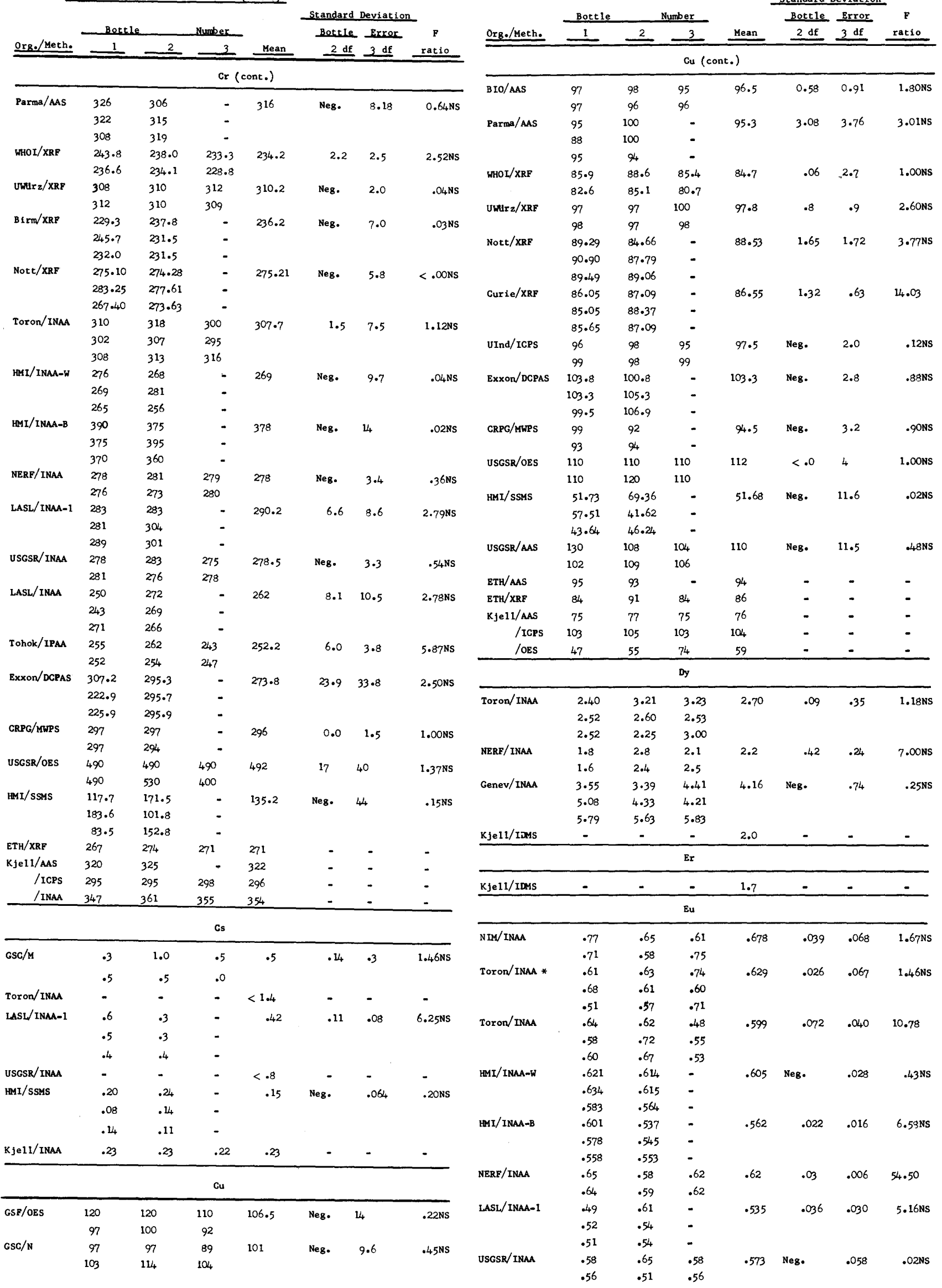


Table 2. Analytical data for USGS-DNC-I (cont.)

\begin{tabular}{|c|c|c|c|c|c|c|c|}
\hline \multirow[b]{3}{*}{ Org./Meth. } & \multirow{3}{*}{$\begin{array}{l}\text { Bottle } \\
1\end{array}$} & \multirow{2}{*}{\multicolumn{2}{|c|}{ Number }} & \multirow[b]{3}{*}{ Mean } & \multicolumn{2}{|c|}{ Standard Deviation } & \multirow{3}{*}{$\begin{array}{c}\text { P } \\
\text { ratio } \\
\end{array}$} \\
\hline & & & & & \multirow{2}{*}{$\frac{\text { Bottle }}{2 \mathrm{df}}$} & \multirow{2}{*}{$\frac{\text { Error }}{3 d f}$} & \\
\hline & & 2 & 3 & & & & \\
\hline \multicolumn{8}{|c|}{ Bu (cont.) } \\
\hline \multirow[t]{3}{*}{ Open/INAM } & 0.63 & 0.61 & - & 0.623 & 0.014 & 0.006 & 18.00 \\
\hline & .63 & .62 & - & & & & \\
\hline & .64 & .61 & - & & & & \\
\hline \multirow[t]{3}{*}{ LASL/INAA } & .77 & .86 & - & .80 & Neg. & .08 & $.50 \mathrm{NS}$ \\
\hline & .68 & .76 & - & & & & \\
\hline & .88 & .85 & - & & & & \\
\hline \multirow[t]{3}{*}{ Che1s/INAA } & .59 & .59 & - & .58 & Neg. & .02 & $.80 \mathrm{NS}$ \\
\hline & .58 & .57 & - & & & & \\
\hline & .55 & .60 & - & & & & \\
\hline \multirow[t]{3}{*}{ Genev/INAA } & .78 & .84 & $1 . \alpha_{4}$ & .92 &.$\alpha_{4}$ & .09 & $1.32 \mathrm{NS}$ \\
\hline & .95 & .96 & .97 & & & & \\
\hline & .94 & .86 & .84 & & & & \\
\hline \multirow[t]{3}{*}{ HMI/SSMS } & 1.27 & 1.20 & - & 1.06 & .059 & .16 & $1.40 \mathrm{NS}$ \\
\hline & 1.16 & .88 & - & & & & \\
\hline & 1.00 & .88 & - & & & & \\
\hline Kjell/IDMS & - & - & - & .5 & - & - & - \\
\hline /INAA & .58 & .59 & .58 & .58 & - & - & - \\
\hline
\end{tabular}

\begin{tabular}{|c|c|c|c|c|c|c|c|}
\hline \multirow[b]{2}{*}{ Org./Methe. } & Botcis & \multicolumn{2}{|r|}{ Number } & \multirow[b]{2}{*}{ Hean } & Standard Deviation & $\begin{array}{l}\text { eviation } \\
\text { Exror }\end{array}$ & \multirow{2}{*}{$\begin{array}{c}F \\
\text { rat10 } \\
\end{array}$} \\
\hline & 1 & 2 & 3 & & $\underline{2 \mathrm{df}}$ & 3 df & \\
\hline \multicolumn{8}{|c|}{ Hf (cont.) } \\
\hline \multirow[t]{2}{*}{ NERF/INAA } & 1.05 & 0.91 & 0.98 & 0.96 & Neg. & 0.078 & $0.05 \mathrm{NS}$ \\
\hline & .90 & 1.01 & .92 & & & & \\
\hline \multirow[t]{3}{*}{ LASL/IMAA-I } & 1.2 & 1.3 & - & 1.12 & .09 & .13 & $2.50 \mathrm{Ns}$ \\
\hline & .9 & 1.2 & - & & & & \\
\hline & 1.0 & 1.1 & - & & & & \\
\hline \multirow[t]{3}{*}{ Open/INAA } & .90 & .93 & - & .93 & Neg. & .05 & $.63 \mathrm{NS}$ \\
\hline & .99 & .95 & - & & & & \\
\hline & .85 & .96 & - & & & & \\
\hline \multirow[t]{2}{*}{ USGSR/IMAA } & 1.1 & 1.0 & 1.0 & 1.02 & .07 &.$\alpha_{4}$ & 7.00ks \\
\hline & 1.1 & 1.0 & .9 & & & & \\
\hline Kjel1/INAA & .9 & .9 & 1.2 & 1.0 & - & - & - \\
\hline
\end{tabular}

\begin{tabular}{llcccccc}
\hline \multicolumn{7}{c}{ P } \\
\hline BMNH & 65 & - & 66 & 66 & - & - & - \\
HMI/SSMS & 51.9 & 50.9 & - & 56.3 & Neg. & 13.6 & $.92 N S$ \\
& 83.7 & 48.5 & - & & & & \\
\multirow{4}{*}{ Munich } & 49.3 & 53.4 & - & & & & \\
& 120 & 115 & - & 115.5 & 4.9 & 7.1 & 2.4 INS \\
& 115 & 117 & - & & & & \\
& 125 & 101 & - & & & & \\
\hline
\end{tabular}

\begin{tabular}{|c|c|c|c|c|c|c|c|}
\hline \multirow[t]{2}{*}{ USGSR/INAA } & .3 & .4 & .4 & .398 & .08 & .09 & $2.60 \mathrm{NS}$ \\
\hline & .3 & .6 & .3 & & & & \\
\hline \multirow[t]{3}{*}{ Chels/INAA } & .48 & .54 & - & .53 & .01 & .03 & $1.54 \mathrm{NS}$ \\
\hline & .55 & .52 & - & & & & \\
\hline & .51 & .58 & - & & & & \\
\hline \multirow[t]{3}{*}{ Genev/INAA } & 2.90 & 2.60 & 1.95 & 2.00 & Neg. & .72 & .3ONs \\
\hline & 1.60 & 1.52 & 1.45 & & & & \\
\hline & 1.65 & 2.72 & 2.30 & & & & \\
\hline
\end{tabular}

\begin{tabular}{|c|c|c|c|c|c|c|c|}
\hline & & & La & & & & \\
\hline \multirow[t]{2}{*}{ NIM/INAA } & 4.9 & 5.3 & $4 \cdot 8$ & 4.77 & .10 & .33 & $1.20 \mathrm{NS}$ \\
\hline & 4.5 & 4.8 & 4.3 & & & & \\
\hline \multirow[t]{2}{*}{ UwUrz/XRF } & 6 & 8 & 6 & 6.3 & .000 & .8 & $1.00 \mathrm{Ns}$ \\
\hline & 6 & 6 & 6 & & & & \\
\hline \multirow[t]{3}{*}{ Birm/XRF } & 4.0 & 3.0 & - & $3 \cdot 3$ & Neg. & .52 & $.02 \mathrm{NS}$ \\
\hline & 3.0 & 3.8 & - & & & & \\
\hline & 2.9 & 3.3 & - & & & & \\
\hline \multirow[t]{3}{*}{ Nott/XRP } & 5.83 & 5.29 & - & 4.82 & .89 & 1.25 & $2.53 \mathrm{Hs}$ \\
\hline & 4.47 & 4.22 & - & & & & \\
\hline & 6.59 & 2.51 & - & & & & \\
\hline Toron/INAA & 3.32 & 3.62 & 3.75 & 3.55 & Neg. & .57 & $.62 \mathrm{~N}$ \\
\hline
\end{tabular}

\begin{tabular}{|c|c|c|c|c|c|c|c|}
\hline \multirow[t]{2}{*}{ GSF/OES } & 22 & 17 & 18 & $15 \cdot 7$ & Neg. & 5.1 & .27NS \\
\hline & 12 & 10 & 15 & & & & \\
\hline \multirow[t]{2}{*}{ UWUrz/XRP } & 12 & 10 & 12 & 11.8 & .4 & .9 & $1.40 \mathrm{Ns}$ \\
\hline & 12 & 12 & 13 & & & & \\
\hline \multirow[t]{3}{*}{ Nott/XRF } & 13.47 & 13.17 & - & 13.47 & .50 & .32 & 8.38 \\
\hline & 13.78 & 13.22 & - & & & & \\
\hline & 4.29 & 12.89 & - & & & & \\
\hline \multirow[t]{3}{*}{ Curle/XRF } & 13.78 & 16.58 & 14.7 & 14.73 & Neg. & 1.26 & $<$.OONS \\
\hline & 15.15 & 13.88 & $=$ & & & & \\
\hline & 15.20 & 13.77 & - & & & & \\
\hline \multirow[t]{2}{*}{ USGSR/OES } & 4 & 13 & 13 & 13.3 & Neg. & .6 & .5 ONS \\
\hline & 13 & 14 & 13 & & & & \\
\hline \multirow[t]{3}{*}{ HMI/SSWS } & 5.53 & 10.84 & - & 6.53 & .61 & 2.25 & $1.22 \mathrm{NS}$ \\
\hline & 5.95 & 4.55 & - & & & & \\
\hline & 5.06 & 7.24 & - & & & & \\
\hline $\mathrm{ETH} / \mathrm{XRF}$ & 17 & 17 & 17 & 17 & - & - & - \\
\hline \multirow[t]{3}{*}{ B1rm/XRF } & 17.5 & 16.9 & - & 16.2 & Neg. & 1.34 & $<$.OONs \\
\hline & 14.3 & 16.5 & - & & & & \\
\hline & 16.6 & 15.1 & - & & & & \\
\hline
\end{tabular}

\begin{tabular}{|c|c|c|c|c|c|c|c|}
\hline & & & Gd & & & & \\
\hline \multirow[t]{3}{*}{ Open/INAA } & 2.8 & 2.0 & - & 2.62 & Neg. & .58 & $<$. .ONS \\
\hline & 2.9 & 2.5 & - & & & & \\
\hline & 2.2 & 3.4 & - & & & & \\
\hline \multirow[t]{2}{*}{ USGSR/INAA } & 1.8 & 1.5 & 1.9 & 1.85 & Neg. & .47 & $.43 \mathrm{NS}$ \\
\hline & 2.2 & 2.4 & 1.3 & & & & \\
\hline \multirow[t]{2}{*}{ KjeIt/IDens } & - & - & - & 1.4 & - & - & - \\
\hline & & & Hf & & & & \\
\hline \multirow[t]{2}{*}{ NDM/INAA } & 1.3 & .8 & 1.2 & 1.1 & Neg. & $\cdot 3$ & $.0 g N S$ \\
\hline & 1.0 & 1.3 & .9 & & & & \\
\hline \multirow[t]{3}{*}{ Toron/INAA } & 1.0 & 1.2 & .9 & 1.02 & Neg. & .19 & $.38 \mathrm{NS}$ \\
\hline & 1.4 & .9 & .9 & & & & \\
\hline & $\cdot 9$ & $\cdot 9$ & 1.1 & & & & \\
\hline \multirow[t]{3}{*}{ HMI/INAA-W } & 1.03 & 1.03 & - & 1.03 & Neg. & .038 & $.07 \mathrm{NS}$ \\
\hline & 1.04 & 1.07 & - & & & & \\
\hline & 1.02 & .965 & - & & & & \\
\hline \multirow[t]{3}{*}{ HMI/INAA-B } & .945 & .885 & - & .912 & .009 & .023 & $1.49 \mathrm{NS}$ \\
\hline & .895 & .924 & - & & & & \\
\hline & .931 & .892 & - & & & & \\
\hline
\end{tabular}

\begin{tabular}{|c|c|c|c|c|c|c|c|}
\hline & 3.60 & 4.17 & 3.85 & & & & \\
\hline & 3.80 & 3.60 & 2.24 & & & & \\
\hline \multirow[t]{3}{*}{ HMI/INRA-W } & 3.45 & 3.16 & - & 3.30 & .103 & .070 & $7.30 \mathrm{Ns}$ \\
\hline & 3.38 & 3.28 & - & & & & \\
\hline & 3.29 & 3.21 & $=$ & & & & \\
\hline \multirow[t]{3}{*}{ HMI/INAA-B } & 3.87 & 3.51 & - & 3.70 & .031 & .11 & $1.22 \mathrm{NS}$ \\
\hline & 3.73 & 3.73 & - & & & & \\
\hline & 3.66 & 3.71 & - & & & & \\
\hline \multirow[t]{2}{*}{ NERP/INAA } & 3.41 & 3.66 & 3.47 & 3.62 & Neg. & .16 & $\cdot 77^{\mathrm{NS}}$ \\
\hline & 3.68 & 3.81 & 3.71 & & & & \\
\hline \multirow[t]{3}{*}{ Open/INAA } & 3.1 & 3.5 & - & 3.62 & Neg. & .45 & $.21 \mathrm{ks}$ \\
\hline & 3.9 & 3.2 & - & & & & \\
\hline & 4.1 & 3.9 & - & & & & \\
\hline \multirow[t]{2}{*}{ USGSR/INAA } & 4 & 5 & 4 & 4 & - & - & - \\
\hline & 4 & 4 & $<1$ & & & & \\
\hline \multirow[t]{3}{*}{ Chels/INAA } & 3.6 & 3.9 & - & 3.80 & .07 & .12 & $2.00 \mathrm{NS}$ \\
\hline & 3.7 & 3.9 & - & & & & \\
\hline & 3.9 & 3.8 & - & & & & \\
\hline \multirow[t]{3}{*}{ Genev/INAA } & 3.42 & 3.79 & 4.37 & 3.84 & .45 & .14 & 21.65 \\
\hline & 3.28 & 4.01 & 4.15 & & & & \\
\hline & 3.20 & 3.15 & 3.20 & & & & \\
\hline \multirow[t]{3}{*}{ Exxon/DGPAS } & 10.1 & 6.9 & - & 10.4 & 2.1 & 1.8 & $5.12 \mathrm{NS}$ \\
\hline & 14.0 & 9.4 & - & & & & \\
\hline & 12.2 & 10.0 & - & & & & \\
\hline \multirow[t]{2}{*}{ KJeI1/ INAA } & 3.44 & 3.45 & 3.48 & 3.46 & - & - & - \\
\hline & & & L1 & & & & \\
\hline \multirow[t]{2}{*}{ GSC/M } & 5 & 6 & 4 & 5.3 & Neg. & 1.4 & $.33 \mathrm{NS}$ \\
\hline & 7 & 4 & 6 & & & & \\
\hline \multirow[t]{2}{*}{ BIO/AAS } & 5.4 & 5.8 & 5.8 & 5.6 & .10 & .12 & $2.33 \mathrm{NS}$ \\
\hline & 5.6 & 5.6 & 5.7 & & & & \\
\hline \multirow[t]{3}{*}{ Parma/AAS } & 5.2 & 4.5 & - & 4.9 & Neg. & .38 & $.73 \mathrm{NS}$ \\
\hline & 5.3 & 4.6 & - & & & & \\
\hline & 4.7 & 5.3 & - & & & & \\
\hline \multirow[t]{2}{*}{ UWUrz/Aus } & 6 & 4 & 7 & 6.0 & 1.4 & .6 & 13.50 \\
\hline & 6 & 5 & 8 & & & & \\
\hline
\end{tabular}


Table 2. Analytical data for USGS-DNG-1 (cont.)

Standard Deviation Bottle Error $F$ 0rg./Meth. 1 Mean 2 2 df 3 df ratio

\begin{tabular}{|c|c|c|c|c|c|c|c|}
\hline & & & Li ( & & & & \\
\hline USGSR/OES & 2 & 2 & 2 & 2.2 & $<0 . \infty 0$ & 0.4 & $1.00 \mathrm{NS}$ \\
\hline & 3 & 2 & 2 & & & & \\
\hline USGSD/AAS & 5 & 5 & 5 & 5 & - & - & - \\
\hline & 5 & 5 & 5 & & & & \\
\hline USGSR/AAS & 4.7 & 5.0 & $4 \cdot 7$ & 4.75 & 0 & .12 & $1.00 \mathrm{NS}$ \\
\hline & 4.7 & 4.7 & 4.7 & & & & \\
\hline & & & Lu & & & & \\
\hline NIM/INAA & .45 & .41 & .46 & .445 & Neg. & .026 & $.07 \mathrm{NS}$ \\
\hline & .44 & .47 & .44 & & & & \\
\hline Toron/INAA & .28 & .31 & .23 & .276 & .032 & .031 & $4.17 \mathrm{NS}$ \\
\hline & .31 & .30 & .25 & & & & \\
\hline & .34 & .23 & .23 & & & & \\
\hline HMI/INAA-W & .362 & .298 & - & .339 & .0093 & .023 & $1.51 \mathrm{NS}$ \\
\hline & .331 & .332 & - & & & & \\
\hline & .357 & .352 & - & & & & \\
\hline HMI-INAA-B & .333 & .329 & - & .332 & .005 & .013 & $1.52 \mathrm{NS}$ \\
\hline & .345 & .341 & - & & & & \\
\hline & .338 & .307 & - & & & & \\
\hline NERF/ INAA & .37 & .31 & .37 & .41 & Neg. & .11 & $.19 \mathrm{NS}$ \\
\hline & .44 & .58 & .38 & & & & \\
\hline Open/INAA & .31 & .30 & - & .33 & Neg. &.$\alpha_{4}$ & $.32 \mathrm{NS}$ \\
\hline & .33 & .41 & - & & & & \\
\hline & .32 & .31 & - & & & & \\
\hline USGSR/INAA & .33 & .33 & .32 & .32 & - & - & - \\
\hline & .32 & .30 & $<.07$ & & & & \\
\hline Chels/INAA & .31 & .34 & - & .33 & .02 & .02 & $3.22 \mathrm{NS}$ \\
\hline & .29 & .36 & - & & & & \\
\hline & .35 & .35 & - & & & & \\
\hline Genev/INAA & .68 & .61 & .70 & .68 & .03 & .06 & $1.61 \mathrm{Ns}$ \\
\hline & .80 & .66 & .63 & & & & \\
\hline & .80 & .63 & .78 & & & & \\
\hline Kjell/INAA & .30 & .30 & .29 & .30 & - & - & - \\
\hline
\end{tabular}

\begin{tabular}{|c|c|c|c|c|c|c|c|}
\hline & & & Mn & & & & \\
\hline \multirow[t]{2}{*}{ B IO/AAS } & 1100 & 1105 & 1115 & 1102 & 3.2 & 7.6 & $1.36 \mathrm{NS}$ \\
\hline & 1090 & 1100 & 1100 & & & & \\
\hline \multirow[t]{3}{*}{ Toron/ INAA } & 1160 & 1230 & 1210 & 1183 & Neg. & 42 & $.08 \mathrm{NS}$ \\
\hline & 1140 & $1 \mu_{4} 0$ & 1190 & & & & \\
\hline & 1230 & 1200 & 1150 & & & & \\
\hline \multirow[t]{2}{*}{ NERF/ INAA } & 1330 & 1430 & 1305 & 1416 & Neg. & 120 & $.26 \mathrm{NS}$ \\
\hline & 1590 & 1,00 & 1440 & & & & \\
\hline \multirow[t]{3}{*}{ LASL/INAA-I } & 1170 & 1180 & - & 1182 & 12.9 & 18.0 & $2.58 \mathrm{NS}$ \\
\hline & 1180 & 1180 & - & & & & \\
\hline & 1160 & 1220 & - & & & & \\
\hline \multirow[t]{2}{*}{ USGSR/OES } & 390 & 800 & 830 & 818 & 122 & 270 & $1.41 \mathrm{NS}$ \\
\hline & 850 & 740 & 1300 & & & & \\
\hline \multirow[t]{4}{*}{$\mathrm{HMI} / \mathrm{SSMS}$} & 758.3 & 1388.1 & - & 907.3 & Neg. & 234 & $.44 \mathrm{NS}$ \\
\hline & 1042.3 & $847 \cdot 2$ & - & & & & \\
\hline & 689.1 & 718.8 & - & & & & \\
\hline & & & Mo & & & & \\
\hline \multirow[t]{2}{*}{ UInd/IGPS } & 1.1 & 1.1 & 1.4 & 1.27 & Neg. & .33 & $.47 \mathrm{NS}$ \\
\hline & 1.2 & 1.8 & 1.0 & & & & \\
\hline \multirow[t]{2}{*}{ USGSR/Spph } & .13 & $\cdot 14_{4}$ & .12 & .127 & .012 & .010 & $4.17 \mathrm{NS}$ \\
\hline & .14 & .13 & .10 & & & & \\
\hline
\end{tabular}

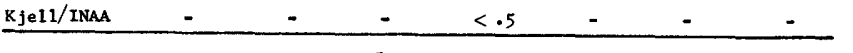

\begin{tabular}{llllllll}
\hline WHOI/XRF & 3.3 & 3.5 & 2.3 & 2.9 & Neg. & .5 & $.49 \mathrm{NS}$
\end{tabular}

UWurz/XRF

Birm/ XRF

$2.8 \quad 2.4 \quad 2.8$

Nott/XRF

$\begin{array}{cc}.8 & .5 \\ 1.1 & .7 \\ .9 & <.4 \\ 5.60 & 5.09 \\ 3.44 & 4.72 \\ 5.81 & 7.49\end{array}$

5.36 Neg. $1.41 \quad .50 \mathrm{NS}$ $5.81 \quad 7.49$

\begin{tabular}{|c|c|c|c|c|c|c|c|}
\hline \multirow[b]{3}{*}{ Org./Meth. } & \multirow[b]{2}{*}{ Bottle } & \multirow{2}{*}{\multicolumn{2}{|c|}{ Number }} & \multirow[b]{3}{*}{ Mean } & \multicolumn{2}{|c|}{ Standard Deviation } & \multirow{3}{*}{$\begin{array}{r}\mathbf{P} \\
\text { ratio } \\
\end{array}$} \\
\hline & & & & & Bottle & Error & \\
\hline & 1 & 2 & 3 & & $\underline{2 \mathrm{df}}$ & $3 \mathrm{df}$ & \\
\hline \multicolumn{8}{|c|}{$\mathrm{Nb}$ (cont.) } \\
\hline \multirow[t]{2}{*}{ Tohok/IPAA } & 3.3 & 3.2 & 2.8 & 2.8 & Neg. & 0.5 & $0.02 \mathrm{NS}$ \\
\hline & 2.2 & 2.5 & 2.7 & & & & \\
\hline \multirow[t]{2}{*}{ USGSR/Spph } & 4.1 & 2.8 & 2.7 & 3.12 & $<.00$ & .57 & $1.00 \mathrm{NS}$ \\
\hline & 2.9 & 3.5 & 2.7 & & & & \\
\hline \multirow[t]{2}{*}{$\mathrm{ETH} / \mathrm{XRF}$} & 3 & 3 & 0 & 2 & - & - & $=$ \\
\hline & & & Nd & & & & \\
\hline $\mathrm{Birm} / \mathrm{XRF}$ & 6.2 & 6.3 & - & 5.8 & Neg. & .59 & $.17 \mathrm{NS}$ \\
\hline & 5.2 & 5.6 & - & & & & \\
\hline & 6.2 & 5.1 & - & & & & \\
\hline Open/INAA & 5.1 & 5.0 & - & 4.83 & Neg. & .66 & $.25 \mathrm{NS}$ \\
\hline & $4 \cdot 3$ & 3.9 & - & & & & \\
\hline & 5.5 & 5.2 & - & & & & \\
\hline USGSR/INAA & 5 & 6 & 5 & 5.0 & $\cdot 3$ & .6 & $1.50 \mathrm{NS}$ \\
\hline & 5 & 5 & 4 & & & & \\
\hline Chels/INAA & 5.2 & 4.6 & - & 5.18 & Neg. & .48 & $.36 \mathrm{NS}$ \\
\hline & $4 \cdot 9$ & 5.4 & - & & & & \\
\hline & $5 \cdot 1$ & 5.9 & - & & & & \\
\hline ETH/XRF & 0 & 2 & 3 & 2 & - & - & - \\
\hline Kjell/IDMs & - & - & - & 4.6 & - & - & - \\
\hline & & & $\mathrm{Ni}$ & & & & \\
\hline GSF/OES & 350 & 300 & 330 & 323.3 & 12 & 14 & $2.30 \mathrm{NS}$ \\
\hline & 330 & 320 & 310 & & & & \\
\hline BMNH/XRF & 230 & 255 & 275 & 264.2 & 15 & 17 & $2.54 \mathrm{NS}$ \\
\hline & 265 & 265 & 295 & & & & \\
\hline $\mathrm{GsC} / \mathrm{N}$ & 260 & 270 & 260 & 258 & 8.2 & 9.1 & $2.60 \mathrm{NS}$ \\
\hline & 240 & 270 & 250 & & & & \\
\hline NIM/AAS & 243 & 242 & 245 & 24,4 & Neg. & 2.9 & $.63 \mathrm{NS}$ \\
\hline & 249 & 24,4 & 242 & & & & \\
\hline Parma/AAS & 272 & 271 & - & 269 & Neg. & 3.65 & .2ONS \\
\hline & 264 & 272 & - & & & & \\
\hline & 269 & 266 & - & & & & \\
\hline WHOI/XRF & 235.9 & 236.4 & 235.2 & 235.6 & 1.1 & 1.1 & $3.04 \mathrm{NS}$ \\
\hline & 237.4 & 235.9 & 233.1 & & & & \\
\hline UWUrz/XRF & 250 & 248 & 252 & 250 & .6 & 1.2 & $1.5 \mathrm{ONS}$ \\
\hline & 250 & 250 & 250 & & & & \\
\hline Liege/XRF & 300 & 290 & - & 285 & Neg. & 11 & $.70 \mathrm{NS}$ \\
\hline & 291 & 284 & - & & & & \\
\hline & 276 & 270 & - & & & & \\
\hline B irm/ XRF & 216.9 & 218.1 & - & 218.2 & Neg. & 1.29 & .2ONs \\
\hline & $218 \cdot 9$ & $217 \cdot 1$ & - & & & & \\
\hline & 218.1 & 220.1 & - & & & & \\
\hline Nott/XRF & 238.35 & 245.31 & - & 241.78 & 4.88 & 1.97 & 19.32 \\
\hline & 239.61 & 243.01 & - & & & & \\
\hline & 236.55 & 247.65 & - & & & & \\
\hline Toron/INAA & - & - & - & $<200$ & - & - & - \\
\hline Tohok/IPAA & 250 & 262 & 267 & 264.2 & 5.5 & 9.6 & $1.66 \mathrm{NS}$ \\
\hline & 260 & 283 & 263 & & & & \\
\hline Exxon/DCPAS & 255.7 & 244.2 & - & 247.6 & 4.5 & 3.0 & 7.88 \\
\hline & 249.6 & $24,4 \cdot 7$ & - & & & & \\
\hline & $247 \cdot 7$ & 243.6 & - & & & & \\
\hline CRPG/MWPS & 278 & 278 & - & 279.8 & Neg. & 2.5 &. $\mathrm{O}_{4} \mathrm{NS}$ \\
\hline & 281 & 282 & - & & & & \\
\hline USGSR/OES & 230 & 250 & 250 & 250 & Neg. & 13 & .30NS \\
\hline & 260 & 250 & 260 & & & & \\
\hline HMI/SSMS & 85.8 & 106.1 & - & 82.8 & Neg. & 25 & $.37 \mathrm{Ns}$ \\
\hline & 114.4 & 59.1 & - & & & & \\
\hline & 66.8 & 64.6 & - & & & & \\
\hline ETH/AAS & 250 & 245 & - & 248 & - & - & - \\
\hline ETH/XRF & 244 & 245 & 239 & 243 & - & - & - \\
\hline Kjell/AAS & 230 & 225 & 223 & 226 & - & - & - \\
\hline / ICPS & 368 & 365 & 371 & 368 & - & - & - \\
\hline /INAA & 228 & 253 & 236 & 239 & - & - & - \\
\hline & & & $\mathrm{Pb}$ & & & & \\
\hline GSF/IDMS & - & - & - & 6.21 & \pm 0.01 & - & - \\
\hline BIO/AAS & - & - & - & $<10$ & - & - & - \\
\hline UWUrz/XRF & 6 & 4 & 4 & 5 & .8 & .8 & $3.00 \mathrm{NS}$ \\
\hline & 6 & 6 & 4 & & & & \\
\hline
\end{tabular}


Table 2. Analytical data for USGS-DNG-1 (cont.)

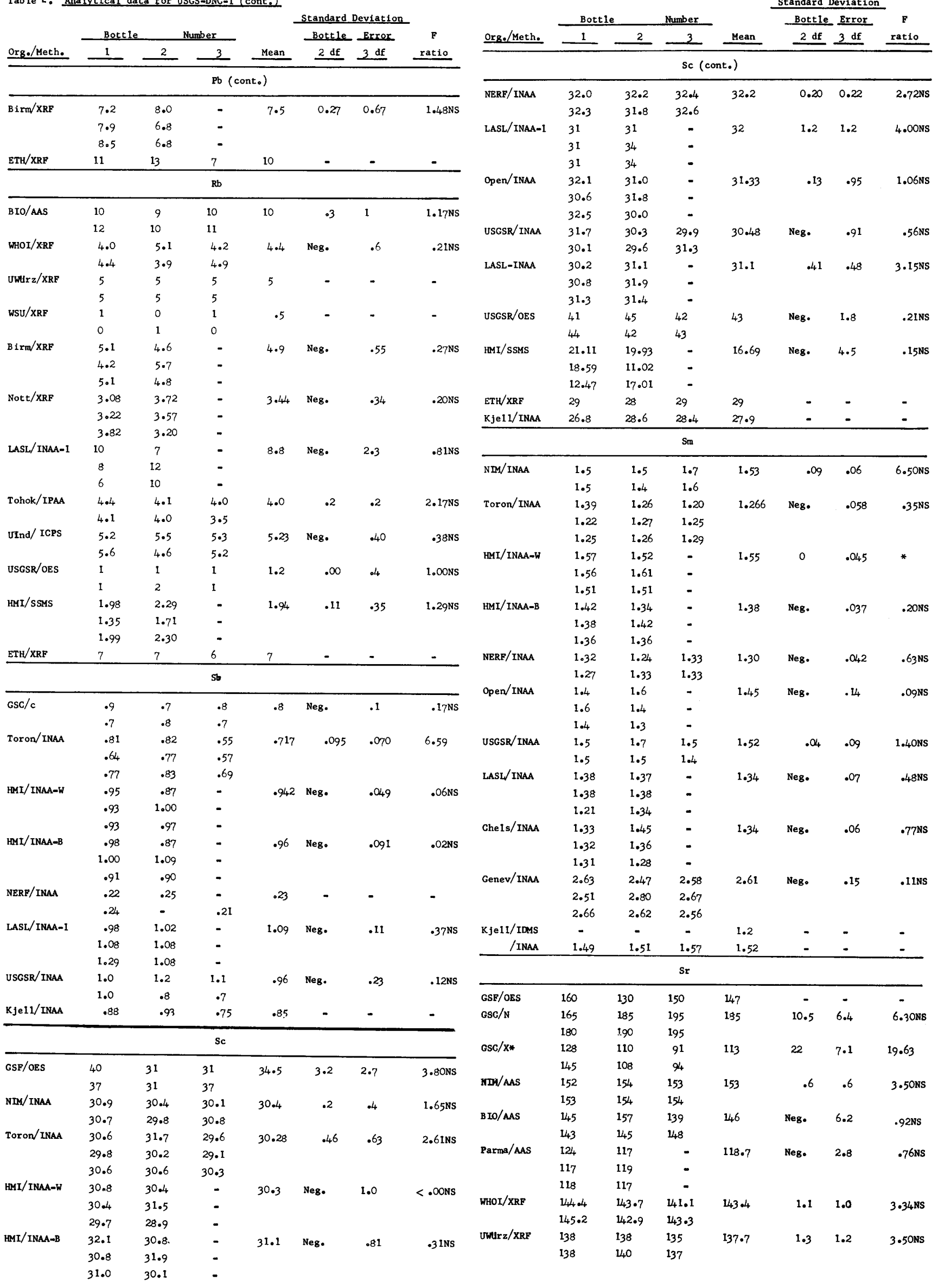


Table 2. Analytical data for USGS-DNC-1 (cont.)

Org./Meth. Bottle Number $\frac{\text { Standard Deviation }}{\text { Bottle Error }}$ F $\underline{1}-2-3$ Mean 2 df 3 df ratio

\begin{tabular}{|c|c|c|c|c|c|c|c|}
\hline \multirow[t]{2}{*}{ WSU/XRF } & 152 & 150 & 148 & 147.8 & Neg. & 3.4 & $0.10 \mathrm{NS}$ \\
\hline & 145 & $\mathrm{u}_{4} 6$ & 146 & & & & \\
\hline \multirow[t]{3}{*}{ Liege/XRF } & $u_{47}$ & 150 & - & $149 \cdot 3$ & Neg. & 1.9 & $.18 \mathrm{NS}$ \\
\hline & 148 & 150 & - & & & & \\
\hline & 152 & 149 & - & & & & \\
\hline \multirow[t]{3}{*}{$\mathrm{Birm} / \mathrm{XRF}_{\mathrm{RF}}$} & 145.1 & 143.2 & - & 143.9 & Neg. & .96 & $.73 \mathrm{NS}$ \\
\hline & 142.8 & 144.1 & - & & & & \\
\hline & $1 / 4.9$ & 143.5 & - & & & & \\
\hline \multirow[t]{3}{*}{ Nott/XRF } & 4.2 .80 & 143.17 & - & 143.00 & .18 & .51 & $1.38 \mathrm{NS}$ \\
\hline & 143.33 & $4+3 \cdot 70$ & - & & & & \\
\hline & $u_{4} 2.15$ & 142.88 & - & & & & \\
\hline \multirow[t]{2}{*}{ Tohok/IPAA } & $u_{45}$ & 48 & 145 & $147 \cdot 7$ & 1.7 & 2.5 & $1.92 \mathrm{NS}$ \\
\hline & 147 & 153 & 148 & & & & \\
\hline \multirow[t]{2}{*}{ UInd/ICPS } & 142 & $v_{4} 2$ & 138 & 140.5 & Neg. & 1.8 & $.63 \mathrm{NS}$ \\
\hline & 141 & 139 & 141 & & & & \\
\hline \multirow[t]{2}{*}{ CRPG/MWPS } & 155 & 152 & - & 153 & $<.0$ & 1.5 & $1.00 \mathrm{NS}$ \\
\hline & 152 & 152 & - & & & & \\
\hline \multirow[t]{2}{*}{ USGSR/OES } & 360 & 400 & 410 & 365 & Neg. & 43 & $.08 \mathrm{NS}$ \\
\hline & 360 & 320 & 340 & & & & \\
\hline \multirow[t]{3}{*}{ HMI/SSMS } & 128.6 & 151.6 & - & 143.1 & Neg. & 17 & $.23 \mathrm{NS}$ \\
\hline & 151.9 & 118.9 & - & & & & \\
\hline & 158.9 & 148.8 & - & & & & \\
\hline ETH/AAS & 146 & 145 & - & 146 & - & - & - \\
\hline $\mathrm{ETH} / \mathrm{XRF}$ & 148 & 146 & 145 & 146 & - & - & - \\
\hline KjeIl/INAA & 100 & 110 & 80 & 97 & - & - & - \\
\hline
\end{tabular}

\begin{tabular}{llllllll}
\multicolumn{9}{c}{ S } \\
\hline ETH $/$ XRF & 597 & 672 & 633 & 634 & - & - & - \\
\hline
\end{tabular}

\begin{tabular}{llllllll}
\multicolumn{7}{c}{ Ta } \\
\hline Toron/INAA & - & - & - & $<.4$ & - & - & - \\
LASL/INAA-1 & .04 & .23 & - & .11 & .02 & .06 & $1.28 \mathrm{NS}$ \\
& .07 & .11 & - & & & & \\
& .13 & .08 & - & & & & \\
Open/INAA & .09 & .09 & - & .09 & $<.00$ & .004 & $1.00 \mathrm{NS}$ \\
& .10 & .09 & - & & & & \\
& .09 & .09 & - & & & & \\
USGSR/INAA & - & - & - & $<.50$ & - & - & - \\
KJe11/INAA & .10 & .08 & .06 & .08 & - & - & - \\
\hline
\end{tabular}

\begin{tabular}{lllllllll}
\hline HMI/INAA-W & .41 & .36 & - & .385 & Neg. & .023 & $.03 \mathrm{NS}$ \\
& .38 & .38 & - & & & &
\end{tabular}

$\begin{array}{llllllll}\text { HMI/INAA-B } & .37 & .41 & - & & & & \\ & .28 & .31 & - & .30 & \text { Neg. } & .018 & 0.45 \mathrm{NS}\end{array}$

\begin{tabular}{|c|c|c|c|c|c|c|c|}
\hline & & & & \multirow[t]{3}{*}{. } & & & \\
\hline & .32 & .29 & - & & & & \\
\hline & .28 & .31 & - & & & & \\
\hline \multirow[t]{2}{*}{ NERF/INAA } & .50 & .46 & .41 & \multirow[t]{2}{*}{.44} & \multirow[t]{2}{*}{.022} & \multirow[t]{2}{*}{.042} & \multirow[t]{2}{*}{$1.54 \mathrm{NS}$} \\
\hline & .47 & .37 & .45 & & & & \\
\hline \multirow[t]{3}{*}{ Open/INAA } & .42 & .42 & - & \multirow[t]{3}{*}{.44} & \multirow[t]{3}{*}{ Neg. } & \multirow[t]{3}{*}{.03} & \multirow[t]{3}{*}{.32NS } \\
\hline & .45 & .41 & - & & & & \\
\hline & .47 & .47 & - & & & & \\
\hline \multirow[t]{2}{*}{ USGSR/INAA } & 47 & .50 & .39 & \multirow[t]{2}{*}{.43} & \multirow[t]{2}{*}{.01} & \multirow[t]{2}{*}{.05} & \multirow[t]{2}{*}{$1.05 \mathrm{NS}$} \\
\hline & .45 & .38 & .39 & & & & \\
\hline \multirow[t]{3}{*}{ CheIs/INAA } & .39 & .38 & - & \multirow[t]{3}{*}{.38} & \multirow[t]{3}{*}{.01} & \multirow[t]{3}{*}{.01} & \multirow[t]{3}{*}{$3.00 \mathrm{NS}$} \\
\hline & .36 & .39 & - & & & & \\
\hline & .36 & .40 & - & & & & \\
\hline $\mathrm{KjelI} / \mathrm{INAA}$ & .37 & .37 & .37 & .37 & - & - & - \\
\hline
\end{tabular}

\begin{tabular}{lccccccc}
\hline \multicolumn{7}{c}{ Th } \\
\hline UWUIrz/XRF & - & - & - & $<1$ & - & - & - \\
Birm/XRF & 1.9 & $<1$ & - & - & - & - & - \\
& 4.1 & $<1$ & - & & & & \\
& 4.0 & 3.2 & - & & & & \\
Toron/INAA & - & - & - & $<.34$ & - & - & - \\
LASL/INAA-1 & .26 & .18 & - & .15 & Neg. & .08 & .06 NS \\
& .17 & .16 & - & & & & \\
& .05 & .09 & - & & & & \\
USGSR/INAA & $<.7$ & .3 & .5 & .4 & - & - & - \\
& .4 & .5 & .4 & & & &
\end{tabular}

\begin{tabular}{|c|c|c|c|c|c|c|c|}
\hline \multirow[b]{3}{*}{ Org./Meth. } & \multirow{2}{*}{\multicolumn{3}{|c|}{ Number }} & \multicolumn{3}{|c|}{ Standard Deviation } & \multirow{3}{*}{$\begin{array}{c}\text { F } \\
\text { ratio } \\
\end{array}$} \\
\hline & & & & \multirow[b]{2}{*}{ Mean } & \multicolumn{2}{|c|}{ Bottle Error } & \\
\hline & 1 & 2 & 3 & & $2 \mathrm{df}$ & $3 \mathrm{df}$ & \\
\hline \multicolumn{8}{|c|}{ Th (cont.) } \\
\hline \multirow[t]{3}{*}{ Open/INAA } & 0.31 & 0.28 & - & 0.28 & Neg. & 0.023 & $0.29 \mathrm{NS}$ \\
\hline & .28 & .25 & - & & & & \\
\hline & .27 & .30 & - & & & & \\
\hline \multirow[t]{2}{*}{ UInd/ICPS } & 1 & 1 & 1 & 1 & - & - & - \\
\hline & 1 & $<1$ & 1 & & & & \\
\hline \multirow[t]{2}{*}{ KjeII/INAA } & .08 & .11 & .07 & .09 & - & - & - \\
\hline & & & Ti & & & & \\
\hline \multirow[t]{2}{*}{ GSE/OES } & 3500 & 3200 & 3400 & 3367 & 101 & 132 & $2.17 \mathrm{NS}$ \\
\hline & 3550 & 3400 & 3150 & & & & \\
\hline Toron/INAA & 2980 & 3040 & 2890 & 2849 & Neg. & 236 & $.74 \mathrm{NS}$ \\
\hline & 2770 & 3050 & 3100 & & & & \\
\hline & 2450 & 2810 & 2550 & & & & \\
\hline USGSR/OES & 24,00 & 2200 & 2200 & 2300 & 82 & 82 & $3.00 \mathrm{NS}$ \\
\hline & 24,00 & 2200 & 24,00 & & & & \\
\hline $\mathrm{HMI} / \mathrm{SSMS}$ & 1455.3 & $2841 \cdot 3$ & - & 1902 & Neg. 56 & 68 & $.51 \mathrm{NS}$ \\
\hline & $2249 \cdot 9$ & 1625.5 & - & & & & \\
\hline & 1508.4 & 1734.8 & - & & & & \\
\hline & & & $\mathrm{T} 1$ & & & & \\
\hline USGSR/AAS & - & - & - & $<.10$ & - & - & - \\
\hline & & & $\mathbf{T m}$ & & & & \\
\hline Open/INAA & .36 & .35 & - & .362 & .024 & .015 & 9.31 \\
\hline & .40 & .34 & - & & & & \\
\hline & .38 & .34 & - & & & & \\
\hline USGSR/INAA & .33 & .37 & .28 & .30 & Neg. & .06 & $.06 \mathrm{NS}$ \\
\hline & .27 & .25 & .30 & & & & \\
\hline Che 1s/INAA & .26 & .28 & - & .30 & .01 & .02 & $1.88 \mathrm{NS}$ \\
\hline & .29 & .33 & - & & & & \\
\hline & .30 & .32 & - & & & & \\
\hline & & & u & & & & \\
\hline Toron/INAA & 2.3 & .7 & 1.5 & 1.37 & Neg. & .80 & .9 INS \\
\hline & 2.3 & .4 & 1.8 & & & & \\
\hline & 1.0 & 2.0 & 1.3 & & & & \\
\hline LASL/DNAA & .10 & .13 & - & .098 & Neg. & .025 & $.68 \mathrm{NS}$ \\
\hline & .11 & .07 & - & & & & \\
\hline & .11 & .07 & - & & & & \\
\hline USGSR/INAA & - & - & - & $<.5$ & - & - & - \\
\hline ETH/XRF & 1 & 0 & 0 & $<1$ & - & - & - \\
\hline Kjell/INAA & .04 & .03 & .05 &.$O_{4}$ & - & - & - \\
\hline & & & $v$ & & & & \\
\hline GSF/OES & 150 & 140 & 150 & $143 \cdot 3$ & 6.5 & 5.8 & $3.50 \mathrm{NS}$ \\
\hline & 40 & 130 & 150 & & & & \\
\hline $\mathrm{GSC} / \mathrm{N}$ & $1_{4} 0$ & 165 & 130 & $1_{45}$ & 9.0 & 8.7 & $3.17 \mathrm{NS}$ \\
\hline & 140 & 150 & 145 & & & & \\
\hline B I0/AAS & 138 & 136 & 146 & $\mathrm{H}_{4} 2$ & Neg. & 4.4 & $.94 \mathrm{NS}$ \\
\hline & 144 & 145 & $1_{4} 6$ & & & & \\
\hline $\mathrm{NIM} / \mathrm{ICPS}$ & 179 & 178 & 160 & 169 & Neg. & 11 & $.08 \mathrm{NS}$ \\
\hline & 164 & 160 & 170 & & & & \\
\hline *Parma/AAS & 170 & 185 & - & 182.5 & Neg. & $7 \cdot 1$ & $.75 \mathrm{NS}$ \\
\hline & 185 & 190 & - & & & & \\
\hline & 185 & 180 & - & & & & \\
\hline *Parma/AAS & 163 & 159 & - & 165 & 2.93 & 4.1 & $2.53 \mathrm{NS}$ \\
\hline & 167 & 164 & - & & & & \\
\hline & 173 & 164 & - & & & & \\
\hline WHOI/XRF & 14.5 .4 & 142.2 & 143.1 & 143.6 & .8 & 1.1 & $2.05 \mathrm{NS}$ \\
\hline & 143.4 & $y_{4} 2.4$ & 144.9 & & & & \\
\hline Nott/XRP & 132.19 & 128.73 & - & 131.35 & 1.93 & 1.72 & $4.79 \mathrm{NS}$ \\
\hline & 133.40 & 132.51 & - & & & & \\
\hline & 133.06 & 128.20 & - & & & & \\
\hline Toron/INAA & 164 & 156 & 165 & 155.4 & Neg. & 8.5 & $.94 \mathrm{NS}$ \\
\hline & 158 & 140 & 14 & & & & \\
\hline & 160 & 158 & 154 & & & & \\
\hline LASL/INAA-I & 139 & 143 & - & $\mathrm{L}_{4} 1.5$ & 8.9 & 9.9 & $3.43 \mathrm{NS}$ \\
\hline & 161 & 133 & - & & & & \\
\hline & 147 & 126 & - & & & & \\
\hline
\end{tabular}




\begin{tabular}{|c|c|c|c|c|c|c|c|}
\hline \multirow[b]{3}{*}{ Org./Meth. } & & & \multirow[b]{2}{*}{ Number } & \multirow[b]{3}{*}{ Mean } & \multicolumn{2}{|c|}{ Standard Deviation } & \multirow{3}{*}{$\begin{array}{c}\mathbf{P} \\
\text { ratio } \\
\end{array}$} \\
\hline & \multicolumn{2}{|c|}{ Bottle } & & & \multirow{2}{*}{$\frac{\text { Bottle }}{2 \mathrm{df}}$} & \multirow{2}{*}{$\frac{\text { Error }}{3 \text { df }}$} & \\
\hline & 1 & 2 & 3 & & & & \\
\hline \multicolumn{8}{|c|}{$v$ (cont.) } \\
\hline \multirow[t]{2}{*}{ UInd/ICPS } & 149 & 49 & 45 & 147.0 & Neg. & 3.1 & $0.2 \mathrm{INS}$ \\
\hline & 147 & 143 & 49 & & & & \\
\hline \multirow[t]{3}{*}{ Exxon/DCPAS } & 149.1 & 140.4 & - & 146.6 & 8.1 & $3 \cdot 7$ & 15.50 \\
\hline & 158.5 & 140.3 & - & & & & \\
\hline & 150.1 & 141.5 & - & & & & \\
\hline \multirow[t]{2}{*}{ CRPG/MWPS } & 184 & 184 & - & 183 & 0 & 1.4 & * \\
\hline & 182 & 182 & - & & & & \\
\hline \multirow[t]{2}{*}{ USGSR/OES } & 200 & 200 & 220 & 200 & Neg. & 28 & $.46 \mathrm{NS}$ \\
\hline & 190 & 230 & 160 & & & & \\
\hline \multirow[t]{3}{*}{ HMI/SSMS } & $75 \cdot 58$ & 119.12 & - & 96.3 & Neg. & 34 & $<.00 \mathrm{NS}$ \\
\hline & 45.14 & 76.99 & - & & & & \\
\hline & 65.84 & 95.05 & - & & & & \\
\hline ETH/XRF & 142 & 137 & $u_{4} 0$ & $v_{40}$ & - & - & - \\
\hline
\end{tabular}

\begin{tabular}{|c|c|c|c|c|c|c|c|}
\hline \multirow[b]{2}{*}{ Org./Meth. } & Bottle & \multicolumn{2}{|r|}{ Number } & \multirow[b]{2}{*}{ Mean } & \multicolumn{2}{|c|}{ Standard Deviation } & \multirow{2}{*}{$\begin{array}{r}F \\
\text { ratio } \\
\end{array}$} \\
\hline & 1 & 2 & 3 & & $\underline{2 \mathrm{df}}$ & $3 \mathrm{df}$ & \\
\hline \multicolumn{8}{|c|}{ Yb } \\
\hline \multirow[t]{3}{*}{ Genev/INAA } & 2.47 & 2.20 & 2.09 & 2.11 & 0.23 & 0.30 & $2.21 \mathrm{NS}$ \\
\hline & 2.20 & 2.31 & 1.41 & & & & \\
\hline & 1.36 & 1.51 & 1.88 & & & & \\
\hline
\end{tabular}

\begin{tabular}{llllllll} 
KjeI1/IDMS & - & - & - & 1.5 & - & - & - \\
/INAA & 1.81 & 1.84 & 1.74 & 1.80 & - & - & - \\
\hline BIO/AAS & 66 & 67 & 65 & 66 & 0.7 & 0.4 & $7.00 N S$
\end{tabular}

\begin{tabular}{llllllll}
\hline USGSR/Spph & .15 & .26 & .11 & .19 & .026 & .049 & $1.54 \mathrm{NS}$ \\
& .18 & .22 & .22 & & & & \\
\hline
\end{tabular}

\begin{tabular}{lllllllll}
\multicolumn{10}{c}{.18} & .22 & .22 \\
\hline GSF/OES & 29 & 28 & 23 & 26.3 & .3 & 2.6 & $1.02 \mathrm{NS}$
\end{tabular}

\begin{tabular}{llllllll}
\hline GSF/OES & 29 & 28 & 23 & 26.3 & .3 & 2.6 & $1.02 \mathrm{NS}$ \\
& 28 & 23 & 27 & & & & \\
WHOI/XRF & 21.3 & 22.2 & 21.6 & 21.3 & Neg. & .8 & $.27 \mathrm{NS}$ \\
& 21.9 & 20.7 & 20.3 & & & &
\end{tabular}

$\begin{array}{llllllll}\text { UWU1 z } / \text { XRF } & 27 & 22 & 25 & 25.2 & 2.2 & .9 & 12.20 \\ & 28 & 24 & 25 & & & & \\ \text { WSU/XRF } & 10 & 15 & 14 & 14.0 & 1.2 & 1.8 & 1.80 \mathrm{NS} \\ & 14 & 15 & 16 & & & & \end{array}$

$\begin{array}{llllllll}\mathrm{Birm} / \mathrm{XRF} & 15.5 & 16.0 & - & 15.9 & \mathrm{Neg} . & .64 & .91 \mathrm{NS}\end{array}$

$\begin{array}{lllllll} & 16.6 & 16.5 & - & & & \\ & 14.9 & 16.0 & - & & & \\ \text { Nott } / \mathrm{XRF} \quad 18.94 & 19.74 & - & 19.92 & & \\ & 18.9 & & \end{array}$

NDM/AAS

$66 \quad 67 \quad 66$

$\begin{array}{llllllll}\text { WHOI/XRF } & 58.8 & 59.7 & 59.3 & 59.3 & \text { Neg. } & .3 & \text {.RANS }\end{array}$

$\begin{array}{lll}59.5 & 59.4 \quad 59.2\end{array}$

$\begin{array}{llllllll}\text { Wurz/XRF } & 65 & 57 & 65 & 62.2 & 3.5 & 1.5 & 12.50\end{array}$

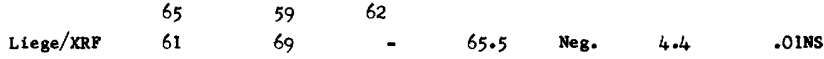

$\begin{array}{llllllll} & 69 & 67 & - & & & & \\ & 67 & 60 & - & & & \\ \text { Birm/ XRF } & 60.8 & 62.5 & - & 61.2 & .39 & .59 & 2.29 \mathrm{NS}\end{array}$

$\begin{array}{llllllll} & 60.6 & 61.2 & - & & & & \\ & 61.2 & 61.1 & - & & & & \\ \text { ote } / \mathrm{XRP} & 67.43 & 66.97 & - & 65.98 & \text { Neg. } & 1.17 & .01 \mathrm{NS}\end{array}$

$\begin{array}{lll}\text { Nott } / \mathrm{XRP} \quad 67.43 & 66.97 \\ & 64.98 & 64.83\end{array}$

$\begin{array}{ll}64.98 & 64.83 \\ 65.71 & 65.99\end{array}$

$\begin{array}{llllllll}\text { Curie/XRF } & 56.58 & 55.67 & - & 57.44 & \text { Nege } & 1.38 & .24 \mathrm{NS}\end{array}$

$\begin{array}{ll}58.96 & 58.77 \\ 57.61 & 57.03\end{array}$

$\begin{array}{llllllll}\text { USGSR/INAA } & 79 & 68 & 70 & 70.5 & \text { Neg. } & 4.9 & .43 \mathrm{NS}\end{array}$

$\begin{array}{llllllll}\text { UInd/ICPS } & 67 & 69 & 70 & & & & \\ & 69 & 68 & 69 & 69.2 & \text { Neg. } & 2.1 & .59 \mathrm{NS}\end{array}$

$\begin{array}{llllllll} & 68 & 73 & 68 & & & & \\ \text { Exxon/DCPAS } & 67.2 & 70.7 & - & 75.4 & 7.8 & 12.6 & 2.13 \mathrm{NS}\end{array}$

$\begin{array}{llllllll}\text { Nott } / \text { XRF } & 18.94 & 19.74 & - & 19.92 & \text { Neg. } & .81 & <.00 N S \\ & 19.90 & 20.56 & - & & & & \end{array}$ $20.94 .19 .46 \quad-$

$\begin{array}{llllllll}\text { Tohok/IPAA } & 18 & 19 & 20 & 18.5 & \text { Neg. } & .9 & .60 \mathrm{NS}\end{array}$

$\begin{array}{lllllllll} & 18 & 18 & 18 & & & & \\ \text { UInd/ICPS } & 18 & 18 & 17 & 17.7 & \text { Neg. } & .6 & .50 \mathrm{NS}\end{array}$

$\begin{array}{lr}66.3 & 703.3 \\ 7.6\end{array}$

$\begin{array}{llllllll}\text { USGSR/OES } & 72 & 83 & 76 & 76 & 6 & 2 & 15.70\end{array}$

$\begin{array}{llllllll}\text { HMI/SSMS } & 67 & 81 & 77 & & & & \\ & 36.18 & 55.98 & - & 38.31 & \text { Neg. } & 12.9 & \text {.OINS }\end{array}$

$\begin{array}{llllllll}\text { USGSR/OES } & 24 & 26 & 26 & 25.2 & .4 & .9 & 1.40 \mathrm{NS}\end{array}$

$\begin{array}{llllllll}\text { HMI } / \text { SSMS } & 25 & 24 & 26 & & & & \\ & 31.6 & 24.8 & - & 27.9 & 8.2 & 2.1 & 44.50\end{array}$

$\begin{array}{lllllll}\text { GCL/AAS } & 28.44 & 33.12 & - & & & \\ 72.5 & 70.0 & 70.0 & 71.7 & \text { Neg. } & 1.4 & .50 \mathrm{NS}\end{array}$

$\begin{array}{lll}72.5 & 72.5 \quad 72.5\end{array}$

$\begin{array}{clllllll}\text { ETH/AAS } & 67.2 & 66.8 & - & 67.0 & - & - & - \\ / \text { XRF } & 67 & 63 & 65 & 65 & - & - & - \\ \text { KJelI/AAS } & 62 & 61 & 61 & 61 & - & - & -\end{array}$

\begin{tabular}{|c|c|c|c|c|c|c|c|c|c|c|c|c|c|c|c|}
\hline \multirow[t]{2}{*}{ ETH/XRF } & \multirow[t]{2}{*}{19} & \multirow[t]{2}{*}{18} & \multirow{2}{*}{$\frac{17}{Y b}$} & \multirow[t]{2}{*}{18} & \multirow[t]{2}{*}{-} & \multirow[t]{2}{*}{ - } & \multirow[t]{2}{*}{ - } & \multirow[t]{2}{*}{$\mathrm{KJelI} / \mathrm{AAS}$} & \multirow[t]{2}{*}{62} & \multirow[t]{2}{*}{61} & \multirow{2}{*}{$\frac{61}{2 x}$} & \multirow[t]{2}{*}{61} & \multirow[t]{2}{*}{ - } & \multirow[t]{2}{*}{ - } & \multirow[t]{2}{*}{ - } \\
\hline & & & & & & & & & & & & & & & \\
\hline \multirow[t]{2}{*}{ NDI/INAA } & 2.8 & 2.9 & 2.7 & \multirow[t]{2}{*}{2.78} & \multirow[t]{2}{*}{ Neg. } & \multirow[t]{2}{*}{.14} & \multirow{2}{*}{$.36 \mathrm{NS}$} & WHOI/XRF & 53.5 & 54.2 & 53.0 & 53.5 & .5 & .2 & 13.42 \\
\hline & 2.9 & 2.6 & 2.8 & & & & & & 53.1 & 54.1 & $53 \cdot 3$ & & & & \\
\hline \multirow[t]{3}{*}{ Toron/INAA } & 1.9 & 2.2 & 2.4 & \multirow[t]{3}{*}{2.12} & \multirow[t]{3}{*}{ Neg. } & \multirow[t]{3}{*}{.17} & \multirow[t]{3}{*}{$.73 \mathrm{NS}$} & UWUrz/XRF & 46 & 50 & 53 & 50.3 & 3.5 & 1 & 25.20 \\
\hline & 2.1 & 1.9 & 2.1 & & & & & & 47 & 52 & 54 & & & & \\
\hline & 2.1 & 2.3 & 2.1 & & & & & WSU/XRP & 29 & 35 & 34 & 32.0 & Neg. & 2.9 & . L8NS \\
\hline \multirow[t]{3}{*}{ HMI/INAA-W } & 1.83 & 1.89 & - & \multirow[t]{3}{*}{1.89} & \multirow[t]{3}{*}{ Neg. } & \multirow[t]{3}{*}{.052} & $.50 \mathrm{NS}$ & & 33 & 30 & 31 & & & & \\
\hline & 1.88 & 1.97 & - & & & & & B $1 \mathrm{rm} / \mathrm{XRF}$ & 37.9 & 39.0 & - & 38.5 & .08 & .47 & $1.0 \mathrm{gNs}$ \\
\hline & 1.91 & 1.85 & - & & & & & & 39.0 & 38.5 & - & & & & \\
\hline RMI/LNAA-B & 1.70 & 1.66 & - & 1.73 & Neg. & .091 & $<.00$ NS & & 38.0 & 38.6 & - & & & & \\
\hline & 1.79 & 1.87 & - & & & & & Nott/XRF & 39.90 & 41.77 & - & 41.90 & .28 & 1.4 & 1. 19NS \\
\hline & 1.70 & 1.67 & - & & & & & & 42.85 & 43.09 & - & & & & \\
\hline NERF/INAA & 2.08 & 2.10 & 1.95 & 2.09 & Neg. & .17 & $.62 N S$ & & 41.41 & 42.35 & - & & & - & \\
\hline & 1.89 & 2.23 & 2.29 & & & & & Tohok/IPAA & 46 & 45 & 44 & 44.2 & Neg. & 1.2 & $.78 \mathrm{NS}$ \\
\hline Open/IMAA & 1.93 & 1.95 & - & 1.976 & .022 & .062 & $1.41 \mathrm{NS}$ & & 44 & 43 & 43 & & & & \\
\hline & 2.10 & 1.96 & - & & & & & USGSR/OES & 36 & 37 & 39 & 37.2 & Neg. & 1.2 & $.78 \mathrm{NS}$ \\
\hline & 1.99 & 1.93 & - & & & & & & 38 & 36 & 37 & & & & \\
\hline USGSR/INAA & 2.0 & 2.0 & 1.9 & 1.9 & - & - & - & HMI/SSMS & 39.0 & 28.6 & - & 39.8 & 8.4 & 5.8 & $7 \cdot 34 \mathrm{NS}$ \\
\hline & 1.9 & 1.9 & $<.5$ & & & & & & 47.2 & $37 \cdot 7$ & - & & & & \\
\hline LASL/INAA & 2.28 & $2 . \alpha_{4}$ & - & 1.95 & Neg. & .35 & $.02 \mathrm{NS}$ & & 52.4 & 34.0 & - & & & & \\
\hline & 1.66 & 2.32 & - & & & & & ETH/XRF & 38 & 37 & 36 & 37 & - & - & - \\
\hline
\end{tabular}

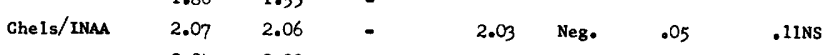

$2.04 \quad 2.00 \quad-$

Tables 1-6 37 
Table 3. Analytical data for USGS-BIR-1

$\left[\mathrm{SiO}_{2}\right.$ through $\mathrm{Fe}_{2} \mathrm{O}_{3} \mathrm{~T}$ in percent; trace elements in parts per million. Org./Meth, organization and method. Details of methods, where available, are given under the organization name at the end of table . A set of data by an organization whose abbreviation is preceded by an asterisk contains data or estimates explained under the organization name. F ratios noted only by an asterisk could not be calculated because of a zero mean square for bottles or error. NS, not significant at the $95 \%$ fractile, $F_{0.05}(2,3)=9.55$. Allowable $F$ ratios for other degrees of freedom (df) and probabilities (p) are given at the end of table for calculated $F$ ratios not followed by NS. Neg., negative bottle variance. $\mathrm{Fe}_{2} \mathrm{O}_{3} \mathrm{~T}$, total $\mathrm{Fe}$ as $\mathrm{Fe}_{2} \mathrm{O}_{3} \cdot 7$

\begin{tabular}{|c|c|c|c|c|c|c|c|}
\hline Org./Meth. & Bottie & \multicolumn{2}{|c|}{ Number } & Mean & $\begin{array}{c}\text { Standard } \\
\text { Bettle } \\
2 \mathrm{df} \\
\end{array}$ & $\begin{array}{l}\text { Exror } \\
3 \mathrm{df} \\
\end{array}$ & $\begin{array}{r}F \\
\text { Ratio } \\
\end{array}$ \\
\hline & & & $\mathrm{SiO}_{2}$ & & & & \\
\hline \multirow[t]{2}{*}{ GSF/Chem } & 47.83 & 47.91 & 47.90 & 47.85 & Neg. & 0.06 & $0.78 \mathrm{NS}$ \\
\hline & 47.79 & 47.77 & 47.89 & & & & \\
\hline \multirow[t]{2}{*}{ *BMNH/Chem } & 48.03 & 48.08 & 48.00 & $47 \cdot 97$ & - & - & - \\
\hline & 48.02 & $47 \cdot 80$ & $47 \cdot 91$ & & & & \\
\hline \multirow[t]{2}{*}{${ }^{* B M N H} / \mathrm{XRF}$} & $47 \cdot 4$ & 48.6 & $48 \cdot 5$ & 48.17 & .74 & .15 & $48 \cdot 50$ \\
\hline & 47.2 & 48.5 & 48.8 & & & & \\
\hline \multirow[t]{2}{*}{ *BMNH/XRF } & 48.11 & 48.38 & 48.24 & 48.08 & Neg. & .24 & $.21 \mathrm{NS}$ \\
\hline & 47.88 & $47 \cdot 90$ & $47 \cdot 99$ & & & & \\
\hline \multirow[t]{2}{*}{$\mathrm{GSC} / \mathrm{A}$} & 48.1 & 48.1 & 48.2 & 48.08 & .08 & .09 & $2.60 \mathrm{NS}$ \\
\hline & 48.0 & $47 \cdot 9$ & 48.2 & & & & \\
\hline \multirow[t]{2}{*}{ Gsc/D* } & 48.01 & 47.91 & 48.52 & 48.29 & .04 & .26 & $1.06 \mathrm{NS}$ \\
\hline & 48.33 & 48.46 & 48.49 & & & & \\
\hline \multirow[t]{2}{*}{ BIO/AAS } & $48 \cdot 16$ & $47 \cdot 30$ & 47.73 & 47.94 & Neg. & .53 & $.33 \mathrm{NS}$ \\
\hline & 48.16 & 48.59 & 47.73 & & & & \\
\hline \multirow[t]{2}{*}{ USGSR/Chem } & 48.0 & 47.7 & 48.1 & 48.02 & Neg. & .17 & $.76 \mathrm{NS}$ \\
\hline & 48.1 & 48.1 & 48.1 & & & & \\
\hline Parma/Chem & 47.50 & 47.43 & - & 47.46 & - & - & - \\
\hline \multirow[t]{2}{*}{ WHOI/XRF } & 47.89 & 48.09 & 48.28 & 48.10 & Neg. & .26 & $.41 \mathrm{NS}$ \\
\hline & 48.44 & 47.83 & 48.06 & & & & \\
\hline \multirow[t]{2}{*}{ WSU/XRF } & $49 \cdot 36$ & 48.67 & 48.59 & 48.75 & .08 & .30 & $1.15 \mathrm{NS}$ \\
\hline & 48.66 & 48.47 & 48.75 & & & & \\
\hline \multirow[t]{2}{*}{ USGSR/XRF } & 48.56 & 48.33 & 48.71 & 48.52 & .13 & .18 & $1.98 \mathrm{NS}$ \\
\hline & 48.20 & 48.59 & 48.74 & & & & \\
\hline *NERF/INAA & 22.29 & 21.64 & 22.02 & 22.23 & Neg. & .76 & $.26 \mathrm{NS}$ \\
\hline & $21 \cdot 54$ & 23.22 & 22.66 & & & & \\
\hline UInd/ICPS & $47 \cdot 4$ & 47.4 & 47.5 & $47 \cdot 57$ & Neg. & .22 & $.23 \mathrm{NS}$ \\
\hline & 47.6 & $47 \cdot 9$ & 47.6 & & & & \\
\hline Exxon/DCPAS & 47.61 & 47.89 & - & 47.96 & Neg. & .23 & $.10 \mathrm{NS}$ \\
\hline & 48.18 & 48.15 & - & & & & \\
\hline & 48.00 & 47.93 & - & & & & \\
\hline CRPG/MWPS & $47 \cdot 90$ & 47.57 & - & 47.75 & .24 & .02 & 345 \\
\hline & 47.93 & 47.59 & - & & & & \\
\hline ETH/AAS & 46.8 & 46.9 & - & 46.85 & - & - & - \\
\hline /XRF & 47.55 & 47.61 & 47.88 & 47.68 & - & - & - \\
\hline *Kjell/XRF & 21.8 & 21.8 & 21.8 & 21.8 & - & - & - \\
\hline & & & $\mathrm{Al}_{2} \mathrm{O}_{3}$ & & & & \\
\hline GSF/Chem & 15.72 & 15.72 & $15 \cdot 72$ & 15.72 & - & - & - \\
\hline & $15 \cdot 72$ & 15.72 & 15.72 & & & & \\
\hline *BMNH/Chem & 16.03 & 16.07 & 15.95 & 16.02 & - & - & - \\
\hline & 16.00 & 16.02 & 16.06 & & & & \\
\hline *BMNH/XRF & 15.3 & 15.6 & 15.4 & $15 \cdot 47$ & .19 & .10 & $8.17 \mathrm{NS}$ \\
\hline & 15.2 & $15 \cdot 7$ & 15.6 & & & & \\
\hline *BMNH/ XRF & 15.65 & $15 \cdot 54$ & 15.71 & 15.66 & .07 & .06 & $3.94 \mathrm{NS}$ \\
\hline & 15.62 & 15.65 & 15.80 & & & & \\
\hline $\mathrm{GSC} / \mathrm{A}^{*}$ & 15.2 & 15.1 & 15.1 & 15.1 & Neg. & .1 & .50 NS \\
\hline & 15.0 & 15.0 & 15.2 & & & & \\
\hline GSG/E & $15 \cdot 70$ & 15.67 & 15.95 & 15.86 & Neg. & .15 & $.5 \mathrm{ONS}$ \\
\hline & 15.94 & 15.96 & 15.95 & & & & \\
\hline B IO/AAS & $15 \cdot 50$ & 15.12 & $15 \cdot 04$ & 15.19 & $\cdot 19$ & .06 & 18.81 \\
\hline & 15.34 & $15 \cdot 12$ & $15 \mathrm{nO}_{4}$ & & & & \\
\hline USGSR/Chem & 15.6 & 15.7 & 15.4 & 15.60 & Neg. & .15 & $.2 \mathrm{INS}$ \\
\hline & 15.7 & 15.5 & 15.7 & & & & \\
\hline Parma/AAS & 15.04 & 15.03 & - & 15.00 & 0 & .093 & * \\
\hline & 15.00 & 15.10 & - & & & & \\
\hline & $14 \cdot 95$ & 14.86 & - & & & & \\
\hline
\end{tabular}

\begin{tabular}{|c|c|c|c|c|c|c|c|}
\hline \multirow[b]{3}{*}{ Org./Meth. } & \multirow[b]{2}{*}{ Boticle } & & & \multirow[b]{3}{*}{ Mean } & \multicolumn{2}{|c|}{ Standard Deviation } & \multirow{3}{*}{$\begin{array}{c}\mathbf{F} \\
\text { ratio } \\
\end{array}$} \\
\hline & & \multicolumn{2}{|c|}{ Number } & & Botite & Error & \\
\hline & 1 & 2 & 3 & & $2 \mathrm{df}$ & $3 \mathrm{df}$ & \\
\hline \multicolumn{8}{|c|}{$\mathrm{Al}_{2} \mathrm{O}_{3}$ (cont.) } \\
\hline \multirow[t]{2}{*}{ WHOI/XRF } & 15.69 & $15 \cdot 70$ & 15.91 & 15.66 & Neg。 & 0.17 & $0.19 \mathrm{NS}$ \\
\hline & $15 \cdot 54$ & 15.62 & 15.53 & & & & \\
\hline \multirow[t]{2}{*}{ UWUIrz/XRF } & 14.6 & $14 \cdot 9$ & 15.2 & 14.97 & Neg。 & .24 & $.74 \mathrm{NS}$ \\
\hline & 15.0 & 15.2 & $14 \cdot 9$ & & & & \\
\hline \multirow[t]{2}{*}{$\mathrm{WSU} / \mathrm{XRF}$} & 16.17 & 16.01 & 16.02 & $15 \cdot 99$ & .08 & .13 & $1.88 \mathrm{NS}$ \\
\hline & 16.06 & $15 \cdot 72$ & 15.96 & & & & \\
\hline \multirow[t]{2}{*}{ USGSR/XRF } & 14.71 & 14.73 & 14.49 & 14.60 & Neg. & .14 & $.93 \mathrm{NS}$ \\
\hline & $14 \cdot 38$ & 14.69 & 14.59 & & & & \\
\hline *Toron/INAA & $7 \cdot 30$ & $7 \cdot 38$ & 7.41 & $7 \cdot 36$ & .049 & .081 & $2.08 \mathrm{NS}$ \\
\hline
\end{tabular}

$+$

*NERF/INAA

$\begin{array}{lll}7.40 & 7.48 & 7.37\end{array}$

$\begin{array}{lll}7.21 & 7.44 & 7.24\end{array}$

$\begin{array}{lll}8.25 & 8.15 & 8.02\end{array}$

*LASL/INAA-1 $8.12 \quad 7.86 \quad-$

$-$

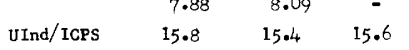

$\begin{array}{lll}15.5 & 15.7 & 15.7\end{array}$

$\begin{array}{llllllllll}\text { Exxon/DCPAS } & 15.38 & 15.65 & - & 15.67 & \text { Neg. } & .20 & .02 N S\end{array}$

$\begin{array}{lllllllll} & 15.94 & 15.77 & - & & & & & \\ & 15.67 & 15.65 & - & & & & \\ \text { CRPG/MWPS } & 15.40 & 15.39 & - & 15.36 & \text { Neg. } & .08 & .87 N S\end{array}$

ETH/AAS $\quad 15.25 \quad 15.40$

\begin{tabular}{lllcllll} 
/XRF & 15.8 & 15.7 & - & 15.75 & - & - & - \\
\hline & 15.65 & 15.64 & 15.72 & 15.67 & - & - & -
\end{tabular}

$\begin{array}{cccccccc}\text { *Xjell/AAS } & 7.80 & 7.70 & 7.50 & 7.66 & - & - & - \\ \text { /ICPS } & 7.59 & 7.61 & 7.77 & 7.66 & - & - & -\end{array}$

\begin{tabular}{llllllll}
$/ \mathrm{XRF}$ & 7.99 & 7.94 & 7.94 & 7.96 & - & - & - \\
\hline & & $\mathrm{Fe}_{2} \mathrm{O}_{3}$ \\
\hline
\end{tabular}

\begin{tabular}{llllllll}
\hline GSF/Chem. & 2.08 & 2.08 & 2.08 & 2.08 & - & - & - \\
GSC/C & 2.08 & 2.08 & 2.08 & & & & \\
& 2.2 & 2.0 & 1.9 & 2.05 & .13 & .04 & 21.00 \\
GSC/F* & 2.2 & 2.0 & 2.0 & & & & \\
& 1.91 & 2.18 & 2.20 & 2.24 & .18 & .37 & $1.46 \mathrm{NS}$ \\
USGSR/Chem & 2.15 & 2.01 & 1.96 & & & & \\
& 2.2 & 2.1 & 1.9 & 2.08 & .16 &.$\alpha_{4}$ & 31.00 \\
ETH/AAS & 2.2 & 2.2 & 1.9 & & & & \\
& 2.03 & 1.96 & - & 2.00 & - & - & - \\
& 1.88 & 1.92 & 2.00 & 1.93 & - & - & - \\
\hline
\end{tabular}

\begin{tabular}{|c|c|c|c|c|c|c|c|}
\hline \multicolumn{8}{|c|}{$\mathrm{FeO}$} \\
\hline GSF/Chem & 8.30 & 8.30 & 8.30 & 8.30 & Neg. & .006 & $.50 \mathrm{NS}$ \\
\hline & 8.30 & 8.29 & 8.29 & & & & \\
\hline
\end{tabular}

*BMNH/Chem $\quad 8.06 \quad 8.42 \quad 8.41 \quad 8.35$

$\begin{array}{lllllllll}\text { GsC/B } & 8.27 & 8.43 & 8.49 & & & & \\ & 8.2 & 8.4 & 8.4 & 8.4 & .10 & .07 & 5.33 \mathrm{NS}\end{array}$

$\begin{array}{llllllll} & 8.3 & 8.5 & 8.5 & & & & \\ \text { GSC/F* } & 8.39 & 8.20 & 8.27 & 8.32 & \text { Neg. } & .10 & .30 \mathrm{NS}\end{array}$

$\begin{array}{llllllll}\text { USGSR/Chem } & 8.4 & 8.3 & 8.4 & 8.37 & \text { Neg. } & .06 & .50 \text { NS }\end{array}$

$\begin{array}{lllcllll}\text { Liege/Vo1 } & 7.99 & 8.4 & 8.3 & & & & \\ & 7.90 & - & 7.85 & \text { Neg. } & .10 & \text { <.OONS }\end{array}$

\begin{tabular}{llllllll}
7.79 & 7.76 & - & & & & \\
ETH/AAS, Co1. & 8.71 & 8.73 & - & 8.72 & - & - & - \\
& 8.68 & 8.63 & 8.57 & 8.63 & - & - & - \\
\hline
\end{tabular}

\begin{tabular}{lllllllll}
\multicolumn{8}{c}{ MgO } \\
\hline GSF/Chem & 9.82 & 9.86 & 9.86 & 9.84 & .010 & .022 & $1.43 \mathrm{NS}$
\end{tabular}

$\begin{array}{ll}9.84 & 9.87 \quad 9.81\end{array}$

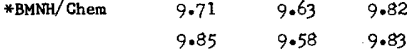

$\begin{array}{lllllllll} & \text { *BMNH } / \text { XRF } & 9.32 & 9.72 & 9.70 & 9.77 & .12 & .27 & 1.39 \mathrm{NS}\end{array}$

$\begin{array}{llllllll}* \mathrm{BMNH} / \mathrm{XRF} & 9.71 & 10.19 & 9.97 & & & & \\ & 9.64 & 9.86 & 9.79 & 9.77 & .10 & .02 & 75\end{array}$

$\begin{array}{llllllll}\text { GSC } / A & 9.67 & 9.86 & 9.82 & & & & \\ & 9.53 & 9.47 & 9.52 & 9.57 & \text { Neg. } & .10 & .82 N S\end{array}$

$\begin{array}{llllllll}\text { GSC/G } & 9.70 & 9.53 & 9.67 & & & & \\ & 9.65 & 9.64 & 9.57 & 9.62 & .04 & .007 & 69\end{array}$ 


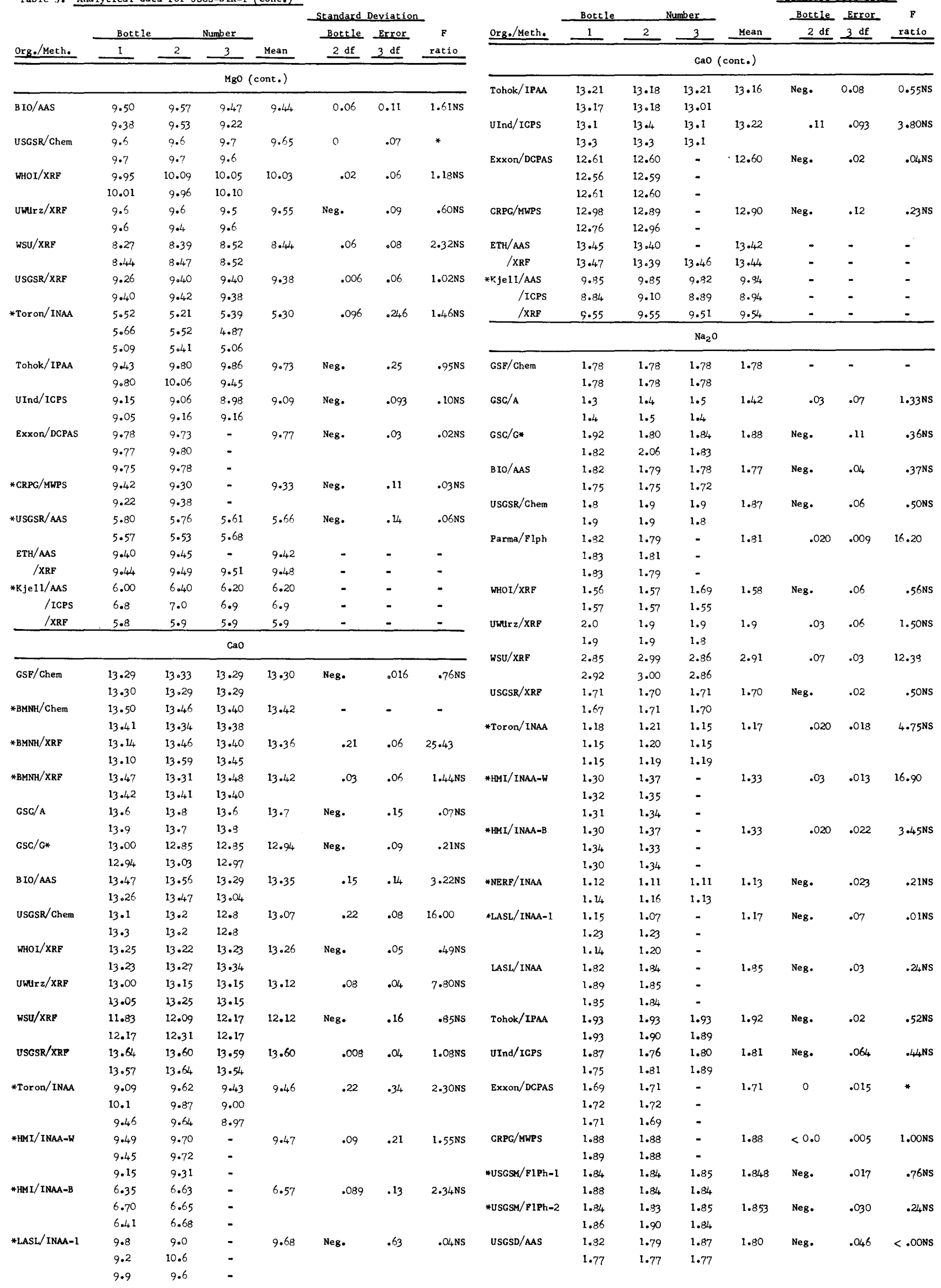


Table 3. Analytical data for USGS-BIR-1 (cont.)

Bottle Number $\quad$ Btandard Deviation Org./Meth. $1 \quad 2 \quad 3 \quad$ Mean 2 df 3 df ratio

\begin{tabular}{llllllll}
\hline \multicolumn{7}{c}{$\mathrm{Na}_{2} \mathrm{O}$ (cont.) } \\
\hline *USGSR/AAS & 1.37 & 1.39 & 1.37 & 1.36 & Neg. & 0.028 & $0.43 \mathrm{NS}$ \\
& 1.39 & 1.33 & 1.34 & & & & \\
ETH/AAS & 1.9 & 1.9 & - & 1.9 & - & - & - \\
/XRF & 1.46 & 1.62 & 1.60 & 1.56 & - & - & - \\
\hline
\end{tabular}

\begin{tabular}{llllllll}
\hline \multicolumn{7}{c}{$\mathrm{x}_{2} \mathrm{O}$} \\
\hline GSF/Chem & .03 & .03 & .03 & .03 & - & - & -
\end{tabular}

$\begin{array}{llllllll}\text { BMNH/AAS } & .03 & .03 & .03 & & & \\ \text { GSC/A } & .016 & .016 & .018 & .017 & - & - & - \\ & - & - & - & <0.0 & - & - & -\end{array}$

$\begin{array}{llllllll}\text { GSC/G* } & .03 & .02 & .03 & .037 & \mathrm{Neg} . & .017 & .39 \mathrm{NS}\end{array}$

$\begin{array}{llllllll} & .03 & .05 & .06 & & & & \\ \text { BIO/AAS } & .01 & .02 & .01 & .01 & <.00<.00 & 1.00 N S\end{array}$

$\begin{array}{llllllll} & .01 & .01 & .01 & & & & \\ \text { USGSR/Chem } & .03 & .03 & .02 & .027 & \text { Neg. } & .006 & .50 N S \\ & .02 & .03 & .03 & & & & \end{array}$

$\begin{array}{llllllll} & .02 & .03 & .03 & & & & \\ \text { Parna/ } / \text { IPh } & .032 & .033 & - & .034 & .002 & .002 & 4.32 \mathrm{NS}\end{array}$

$\begin{array}{llllllll} & .032 & .039 & - & & & & \\ & .033 & .036 & - & & & & \\ \text { WHOI } / \mathrm{XRP} & .006 & .009 & .008 & .008 & \text { Neg. } & .002 & .67 \mathrm{NS}\end{array}$

$\begin{array}{llllllll} & .006 & .009 & .008 & .008 & \text { Neg. } & .002 & .67 \text { NS } \\ \text { UWUizz/XRF } & .010 & .009 & .006 & & & & \\ & .05 & .04 & .04 & .043 & .006 & 0 & *\end{array}$

$\begin{array}{lllllllll} & .05 & .04 & .04 & & & & \\ \text { wSU } / \mathrm{XRF} & .03 & .03 & .02 & .033 & .009 & .013 & 1.90 \mathrm{NS}\end{array}$

$\begin{array}{llllllll} & .04 & .06 & .02 & & & & - \\ \text { USGSR/XRF } & .07 & .07 & .07 & .07 & - & - & - \\ & .07 & .07 & .07 & & & & \end{array}$

$\begin{array}{llllllll}\text { UInd/ICPS } & .05 & .03 & .01 & .032 & .017 & .004 & 37.00\end{array}$

$\begin{array}{llllllll} & .05 & .03 & .02 & & & & \\ \text { Exxon/DGPAS } & .03 & .03 & - & .032 & <.000 & .004 & 1.00 \mathrm{NS}\end{array}$

$\begin{array}{lllllllll} & .04 & .03 & - & & & & & \\ & .03 & .03 & - & & & & \\ \text { CRPG/MWPS } & .04 & .03 & - & .03 & .005 & .007 & 2.00 N S\end{array}$

$\begin{array}{llllllll} & .03 & .02 & - & & & & \\ \text { USGSH/PIPh-1 } & .022 & .023 & .019 & .021 & \text { Neg. } & .0025 & .03 \text { NS }\end{array}$

$\begin{array}{llllllll} & .020 & .020 & .024 & & & & \\ \text { USGSM/F1Ph-2 } & .021 & .019 & .017 & .031 & .005 & .026 & 1.06 \mathrm{NS}\end{array}$

$\begin{array}{lllllllll} & .085 & .025 & .019 & & & & \\ \text { *USGSR/AAS } & .017 & .017 & .017 & .0175 & \text { Neg. } & .001 & .60 \mathrm{NS} \\ & .017 & .019 & .018 & & & & \end{array}$

\begin{tabular}{|c|c|c|c|c|c|c|c|}
\hline ETH/AAS & .03 & .03 & - & $<.03$ & - & - & - \\
\hline /XRF & - & - & - & .01 & - & - & - \\
\hline *KjeIl/XRF & - & - & - & $<.04$ & - & - & - \\
\hline \multirow[t]{2}{*}{ HMMN/XRF-3 } & .031 & .026 & .018 & .027 & Neg. & .006 & $.13 \mathrm{NS}$ \\
\hline & .025 & .029 & .032 & & & & \\
\hline \multicolumn{8}{|c|}{$\mathrm{H}_{2} \mathrm{O}^{+}$} \\
\hline GSF/Chem & .06 & .06 & .06 & .063 & Neg. & .006 & $.50 \mathrm{NS}$ \\
\hline
\end{tabular}

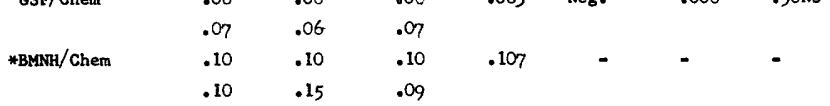

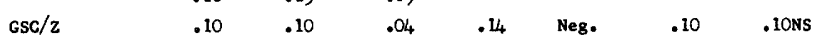

$\begin{array}{llllllll} & .32 & .12 & .14 & & & & \\ \text { GSC/Y* } & .02 & .02 & .07 & .04 & .02 & .02 & 3.07 N S\end{array}$

$\begin{array}{lllllllll} & .05 & .04 & .06 & & & & & \\ \text { USGSR/Chem } & .25 & .33 & .30 & .29 & .04 & 0\end{array}$

$\begin{array}{llllllll}\text { Liege/Grav } & .25 & .33 & .30 & & & & \\ & .08 & .07 & - & .078 & .028 & .022 & 6.04 \mathrm{NS}\end{array}$

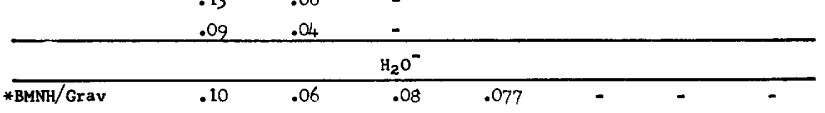

$\begin{array}{llllllll}\text { GSC/b } & .05 & .08 & .09 & & & & \\ & .10 & .10 & .06 & .08 & .02 & .01 & 4.50 N S \\ \text { USGSR/Grav } & .08 & .08 & .06 & & & & \\ & .00 & .02 & .02 & .013 & .012 & 0 & * \\ \text { Liege/Grav } & .00 & .02 & .02 & & & & \\ & .09 & .07 & - & .142 & \text { Neg. } & .10 & .02 N S \\ & .21 & .28 & - & & & & \end{array}$

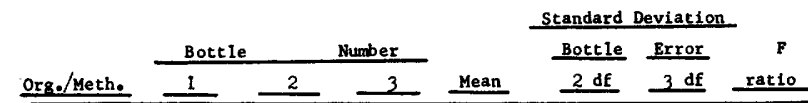

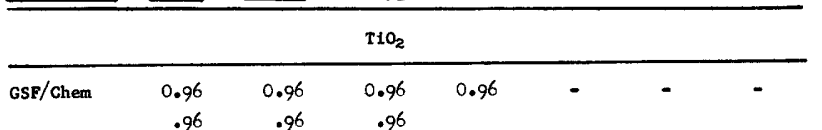

$\begin{array}{llll}0.96 & .96 & .96\end{array}$

$\begin{array}{lll}.99 & .91 & .98\end{array}$

$\begin{array}{llllllll}* \mathrm{BMNH} / \mathrm{XRF} & .90 & .92 & .92 & .918 & .015 & .009 & 6.20 \mathrm{NS} \\ & .90 & .93 & .94 & & & & \\ \text { *BMNH } / \mathrm{XRF} & .918 & .926 & .924 & .921 & .003 & .004 & 2.46 \mathrm{NS} \\ & .915 & .917 & .927 & & & & \end{array}$

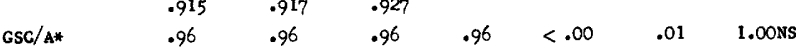

$\begin{array}{llllllll}\text { GsC/h } & .97 & .96 & .89 & .92 & .04 & .02 & 13.00\end{array}$

BIO/AAS $\quad .94 \quad .96 \quad .87$

$\begin{array}{llllllll}.93 & .90 & .90 & .94 & \text { Neg. } & .04 & .08 N S\end{array}$

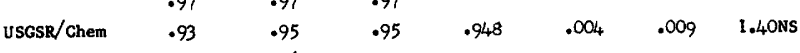

$\begin{array}{lllllll}\text { Parma/Color } & .95 & .980 & .96 & .95 & & \\ & .980 & - & .983 & \text { Neg. } & .019 & .13 \text { NS }\end{array}$

$\begin{array}{rr}.963 & .988 \\ 1.04 & .972\end{array}$

$\begin{array}{lllllllll}\text { WHOI } / \mathrm{XRF} & .960 & .966 & .965 & .954 & \mathrm{Neg} . & .003 & .04 \mathrm{NS}\end{array}$

$\begin{array}{llllllll}.96 & .96 & .96 & .96 & .006 & .012 & 1.50 \mathrm{NS}\end{array}$

WSU $/$ XRF $\quad .944$

USGSR/XRF $\quad .94 \quad .94$

$\begin{array}{lll}.93 & .95 \\ \text { LASL/INAA-1 } & .70 & .56\end{array}$

$.57 \quad .56$

Tohok/IPAA $\quad .53 \quad .02 \quad . .00$

$\begin{array}{rrrrrrrr} & 1.02 & 1.02 & 1.01 & & & & \\ \text { UInd/ICPS } & .95 & .96 & .94 & .952 & .010 & .004 & 13.00\end{array}$

$\begin{array}{llllllll} & .96 & .96 & .94 & & & & \\ \text { Exxon/DCPAS } & .99 & .97 & - & .98 & .003 & .006 & 2.00 \mathrm{NS}\end{array}$

$\begin{array}{rll}.99 & .97 \\ & .98\end{array}$

$\begin{array}{lrrrrrr} & .98 & .98 & - & & & \\ \text { GRPG/MWPS } & 1.01 & 1.00 & - & 1.01<.0 & .01<.00 \mathrm{NS}\end{array}$

$1.01 \quad 1.02$

$\begin{array}{lll}\text { XRF } & .97 \quad .96\end{array}$

*Kjell/ IGPS $\quad .58 \quad .58$

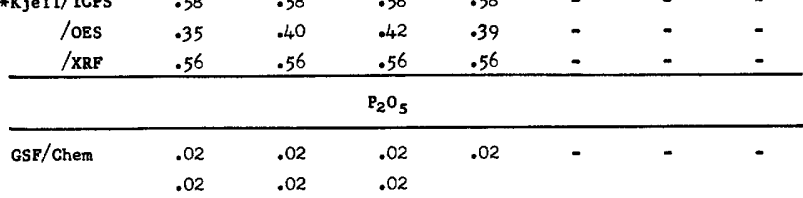

*BMMH/Chem $\quad .04 \quad 02 \quad .02 \quad .06$

$\begin{array}{lll}.02 & .06 \quad .02\end{array}$

*BMNH/XRF-1 $\quad .02 \quad .02 \quad .02 \quad .02$

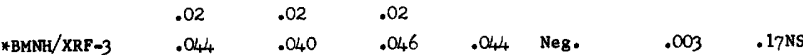

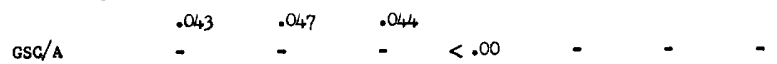

Gsc/J* $\quad \begin{array}{llllll}.02 & .02 & .02 & .02 & - & -\end{array}$

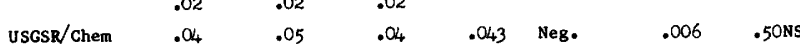

Parma/Color $\begin{array}{lllllll}.021 & .028 & - & .023 & .0009 & .002 & 1.44 N S\end{array}$

$\begin{array}{llllllll} & .022 & .022 & - & & & & \\ \text { WHOI } / \text { XRF } & .101 & .097 & .096 & .097 & \text { Neg. } & .003 & .17 \mathrm{NS}\end{array}$

$\begin{array}{llllllll}\text { UWurz } / \text { XRF } & .095 & .096 & .098 & & & \\ & .06 & .07 & .07 & .055 & \text { Neg. } & .017 & .53 \text { NS }\end{array}$

WSU/XRF $\quad .03 \quad .03 \quad .03$

$\begin{array}{llll}\text { USGSR XRF } & .02 & .04 & .04 \\ & .05 & .04 & .04\end{array}$ 
Table 3. Analytical data for USGS-BIR-I (cont.)

\begin{tabular}{|c|c|c|c|c|c|c|c|c|c|c|c|c|c|c|c|}
\hline \multirow{3}{*}{ Org./Meth. } & \multirow{3}{*}{$\begin{array}{c}\text { Bottle } \\
1 \\
\end{array}$} & \multirow{2}{*}{\multicolumn{2}{|c|}{ Number }} & \multirow[b]{3}{*}{ Mean } & \multicolumn{2}{|c|}{ Standard Deviation } & \multirow{3}{*}{$\begin{array}{c}F \\
\text { ratio } \\
\end{array}$} & & Bott 1 & & nber & & Bottle & Error & $\mathbf{F}$ \\
\hline & & & & & Bottle & Error & & org./Meth. & 1 & 2 & 3 & Mean & $\underline{2 d f}$ & $3 \mathrm{df}$ & ratio \\
\hline & & 2 & 3 & & $2 \mathrm{df}$ & $3 \mathrm{df}$ & & & & & $\mathrm{Fe}_{2} \mathrm{O}_{3} \mathrm{~T}$ & & & & \\
\hline & & & $\mathrm{P}_{2} \mathrm{O}_{5}(\mathrm{c}$ & int.) & & & & 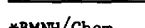 & 11 & $11 ? 1$ & $11,1 / 4$ & 11.29 & - & 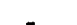 & $=$ \\
\hline UInd/ICPS & 0.21 & 0.21 & 0.15 & 0.175 & Neg. & 0.033 & $0.32 \mathrm{NS}$ & & 11.35 & 11.37 & 11.37 & & & & \\
\hline & .15 & .16 & .17 & & & & & *BMNH/XRF-1 & 11.06 & 11.25 & 11.30 & 11.21 & 0.138 & 0.014 & 190 \\
\hline Exxon/DCPAS & .033 & .021 & - & .027 & Neg. & .004 & $.42 \mathrm{NS}$ & & 11.04 & 11.27 & 11.32 & & & & \\
\hline & .028 & .028 & - & & & & & *BMMH/ $/ \mathrm{XRF}-3$ & 11.18 & 11.05 & 11.10 & 11.11 & .039 & .026 & $5.45 \mathrm{NS}$ \\
\hline & .024 & .029 & - & & & & & & 11.13 & 11.09 & 11.09 & & & & \\
\hline ETH/AAS & .1 & .104 & - & .102 & - & - & - & $\mathrm{GSC} / \mathrm{A}$ & 11.3 & 11.3 & 11.2 & 11.33 & Neg. & .1 & $.17 \mathrm{NS}$ \\
\hline /XRF & .04 & .04 & .04 & .04 & - & - & - & & 11.4 & 11.4 & 11.4 & & & & \\
\hline & & & Mno & & & & & GSC/F* & 11.23 & 11.29 & 11.39 & 11.31 & Neg. & .08 & $.05 \mathrm{NS}$ \\
\hline & & & (n) & & & & & & 11.37 & 11.30 & 11.26 & & & & \\
\hline GSE/Color & .17 & .17 & .17 & .17 & - & - & - & B IO/ AAS & 11.32 & 11.18 & 11.12 & 11.14 & .07 & .10 & $1.82 \mathrm{NS}$ \\
\hline & .17 & .17 & .17 & & & & & & 11.15 & 11.09 & 10.95 & & & & \\
\hline BMNH/Golor & .176 & - & .174 & .175 & - & - & - & Parma/Color & 11.32 & 11.46 & - & 11.42 & .036 & .081 & $1.58 \mathrm{NS}$ \\
\hline *BMNH/XRF-1 & .17 & .17 & .17 & .17 & - & - & - & & 11.46 & 11.56 & - & & & & \\
\hline & .17 & .17 & .17 & & & & & & 11.37 & 11.38 & - & & & & \\
\hline *BMNH $/$ XRF-3 & .170 & .170 & .169 & .170 & Neg. & .0009 & $.20 \mathrm{NS}$ & WHOI $/ X R F$ & 11.36 & 11.41 & 11.35 & 11.38 & Neg. & .065 & $.30 \mathrm{NS}$ \\
\hline & .170 & .171 & .171 & & & & & & 11.39 & 11.30 & 11.46 & & & & \\
\hline $\mathrm{GSC} / \mathrm{A*}$ & .17 & .18 & .18 & .178 & Neg. & .009 & $.20 \mathrm{NS}$ & UWUrz/XRF & 11.30 & 11.45 & 11.35 & 11.38 & .074 & .020 & 27.00 \\
\hline & .19 & .17 & .18 & & & & & & 11.30 & 11.45 & 11.40 & & & & \\
\hline GSC/G* & .17 & .17 & .17 & .173 & Neg. & .006 & $.50 \mathrm{NS}$ & $\mathrm{WSU} / \mathrm{XRF}$ & 10.98 & 11.32 & 11.31 & 11.28 & .15 & .16 & $2.77 \mathrm{NS}$ \\
\hline & .18 & .18 & .17 & & & & & & 11.22 & 11.53 & 11.31 & & & & \\
\hline $\mathrm{GSC} / \mathrm{N}$ & .22 & .22 & .21 & .222 & .004 & .009 & $1.40 \mathrm{NS}$ & USGSR/XRF & 11.25 & 11.48 & 11.40 & 11.31 & Neg. & .13 & $.21 \mathrm{NS}$ \\
\hline & .22 & .24 & .22 & & & & & & 11.27 & 11.20 & 11.25 & & & & \\
\hline USGSR/Chem & .21 & .20 & .20 & .197 & Neg. & .01 & .17 NS & *Toron/INAA & $7 \cdot 82$ & $7 \cdot 80$ & 8.17 & 8.06 & $.10_{4}$ & .176 & $2 . \mathrm{O}_{4} \mathrm{NS}$ \\
\hline & .19 & .19 & .19 & & & & & & 8.15 & 8.18 & 8.26 & & & & \\
\hline WHOI/XRF & .172 & .175 & .173 & .172 & Neg. & .002 & $.92 \mathrm{NS}$ & & 8.17 & 7.77 & 8.19 & & & & \\
\hline & .171 & .172 & .171 & & & & & *HMI/INAA-W & 8.00 & 8.27 & - & 8.02 & .093 & .17 & $1.92 \mathrm{NS}$ \\
\hline Uwurz/XRF & .16 & .16 & .16 & .16 & - & - & - & & 7.87 & 8.21 & - & & & & \\
\hline & .16 & .16 & .16 & & & & & & 7.89 & 7.85 & - & & & & \\
\hline WSU/XRF & .16 & .17 & .16 & .163 & .006 & 0 & * & *HMI/INAA-B & $7 \cdot 76$ & 8.20 & - & 7.86 & .15 & .16 & $3.66 \mathrm{NS}$ \\
\hline & .16 & .17 & .16 & & & & & & 7.79 & $7 \cdot 96$ & - & & & & \\
\hline USGSR/XRP & .17 & .17 & .17 & .17 & - & - & - & & 7.65 & $7 \cdot 78$ & - & & & & \\
\hline & .17 & .17 & .17 & & & & & * NERF/INAA & 8.21 & 8.29 & 8.15 & 8.26 & .081 & .084 & $2.84 \mathrm{NS}$ \\
\hline Tohok/IPAA & .170 & .177 & .177 & .176 & Neg. & .008 & $.40 \mathrm{NS}$ & & 8.16 & 8.45 & 8.27 & & & & \\
\hline & .187 & .177 & .166 & & & & & *LASL/INAA-1 & 7.59 & $7 \cdot 73$ & - & $7 \cdot 69$ & .06 & .13 & $1.73 \mathrm{NS}$ \\
\hline UInd/ICPS & .16 & .16 & .15 & .158 & $<.000$ & .004 & $1.00 \mathrm{NS}$ & & 7.82 & 7.61 & - & & & & \\
\hline & .16 & .16 & .16 & & & & & & 7,86 & $7 \cdot 52$ & - & & & & \\
\hline Exxon/DCPAS & .18 & .18 & - & .18 & - & - & - & Open/INAA & 11.35 & 11.77 & - & 11.15 & Neg. & .44 & - IgNS \\
\hline & .18 & .18 & - & & & & & & 11.06 & 10.67 & - & & & & \\
\hline & .18 & .18 & - & & & & & & 11.28 & 10.78 & - & & & & \\
\hline CRPG/MWPS & .16 & .14 & - & .15 & 0 & .01 & * & *USGSR/INAA & 7.89 & 7.81 & 7.11 & $7 \cdot 75$ & Ne8. & .44 & . I2NS \\
\hline & .14 & .16 & - & & & & & & 7.65 & 7.89 & 8.16 & & & & \\
\hline ETH/AAS & .168 & .168 & - & .168 & - & - & - & LASL/INAA & 11.36 & 11.37 & - & 11.35 & Neg. & .15 & $.89 \mathrm{NS}$ \\
\hline /XRF & .17 & .17 & .17 & .17 & - & - & - & & 11.56 & 11.10 & - & & & & \\
\hline *Kjell/AAS & .1250 & .1250 & .1240 & .1246 & - & - & - & & 11.30 & 11.40 & - & & & & \\
\hline /ICPS & .1453 & .1490 & .4885 & .476 & - & - & - & Tohok/IPAA & 11.55 & 11.49 & 11.31 & 11.51 & .11 & .09 & $4.12 \mathrm{NS}$ \\
\hline & & & $\mathrm{CO}_{2}$ & & & & & & 11.72 & 11.55 & 11.54 & & & & \\
\hline & & & & & & & & UInd/ICPS & 11.3 & 11.5 & 11.3 & 11.38 & .11 & .09 & $3.80 \mathrm{NS}$ \\
\hline *BMNH/Chem & .00 & .07 & .04 & .03 & - & - & - & & 11.5 & 11.5 & 11.2 & & & & \\
\hline & .00 & .05 & .03 & & & & & Exx on/DCPAS & 11.16 & 11.28 & - & 11.37 & Neg. & .14 & $.03 \mathrm{NS}$ \\
\hline $\mathrm{GsC} / \mathrm{a}$ & .0 & .0 & .0 & .03 & Neg. & .06 & $.50 \mathrm{NS}$ & & 11.52 & 11.45 & - & & & & \\
\hline & .1 & .1 & .0 & & & & & & 11.39 & 11.40 & - & & & & \\
\hline $\operatorname{Gsc} / \mathrm{Y}^{*}$ & .03 & .04 & .03 & .03 & .003 & .006 & $1.50 \mathrm{NS}$ & CRPG/MWPS & 11.30 & 11.38 & - & 11.36 & .10 & .05 & $7 \cdot 72 \mathrm{NS}$ \\
\hline & .02 & .03 & .03 & & & & & & 11.27 & 11.48 & - & & & & \\
\hline USGSR/Chem & .01 & .01 & .01 & .01 & - & - & - & *Kjell/Aas & $7 \cdot 80$ & 8.00 & 8.00 & $7 \cdot 93$ & - & - & - \\
\hline & .01 & .01 & .01 & & & & & /ICPS & 7.09 & 7.82 & 7.86 & 7.59 & - & - & - \\
\hline & & & G1 & & & & & /INAA & $7 \cdot 14$ & 6.93 & 7.41 & $7 \cdot 16$ & - & - & - \\
\hline & & & & & & & & $/ \mathrm{XRF}$ & 7.76 & 7.83 & 7.83 & 7.81 & - & - & - \\
\hline $\mathrm{GSC} / \mathrm{L}$ & $\begin{array}{l}.04 \\
.01\end{array}$ & $\begin{array}{l}.07 \\
.01\end{array}$ & $\begin{array}{l}.01 \\
.01\end{array}$ & .02 & Neg. & .03 & $.60 \mathrm{Ns}$ & & & & $A_{B}$ & & & & \\
\hline & & & $\mathbf{F}$ & & & & & USGSR/AAS & .036 & .033 & .044 & .039 & .002 & .009 & $1.13 \mathrm{NS}$ \\
\hline $\mathrm{GSC} / \mathrm{K}$ & .03 & .03 & .02 & .02 & Neg. & .006 & $.50 \mathrm{NS}$ & & .056 & .032 & .035 & & & & \\
\hline & .02 & .02 & .02 & & & & & & & & As & & & & \\
\hline & & & L.O.I. & & & & & $\mathrm{GSC} / \mathrm{c}$ & - & - & - & $<.2$ & - & - & - \\
\hline UInd/Grav & - & - & .05 & - & - & - & - & Toron/INAA & - & - & - & $<2$ & - & - & - \\
\hline $1 /$ & & & & $=0$ & & $0 \%$ & & GCL/Spph & .6 & 1.2 & .5 & .98 & Neg. & .38 & .5 INS \\
\hline CRPG/Grav & -.55 & -.047 & - & -.50 & Neg. & .05 & $.09 \mathrm{NS}$ & & 1.2 & 1.2 & 1.2 & & & & \\
\hline & -.46 & -.51 & - & & & & & Kjell/INAA & .3 & $\cdot 3$ & $\cdot 3$ & .3 & - & - & - \\
\hline
\end{tabular}


Table 3. Analytical data for USGS-BIR-1 (cont.)

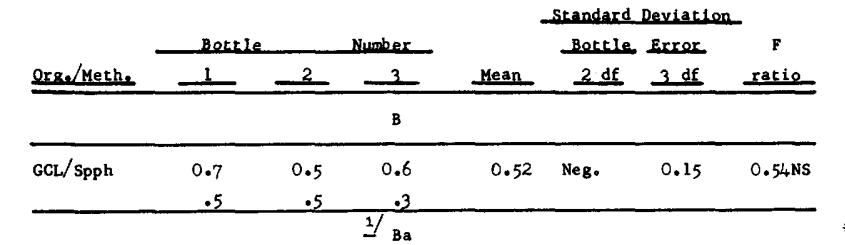

\begin{tabular}{lccccccc}
\hline GSC/N & 8 & 9 & 9 & 10.5 & Neg. & 3.1 & $.60 \mathrm{NS}$ \\
WHOI/XRF & 11 & 16 & 10 & & - & & \\
UWUrz/XRF & 16 & 22 & 14 & 17.7 & 3.9 & .6 & 13.00 \\
& 17 & 22 & 15 & & & & \\
Birm/ XRF & 16.6 & 17.0 & - & 11.8 & Neg. & 4.60 & $.09 N S$
\end{tabular}

\begin{tabular}{cccccccc} 
& 10.4 & 10.4 & - & & & & \\
\multirow{3}{*}{ Nott/XRF } & 6.6 & 9.5 & - & & & & \\
& 31.95 & 32.84 & - & 33.75 & Neg. & 1.33 & .51 NS \\
& 34.95 & 34.70 & - & & & &
\end{tabular}

\begin{tabular}{lccccccc} 
& 33.17 & 34.86 & - & & & & \\
Open/INAA & - & - & - & 4 & - & - & - \\
UInd/ICPS & 6 & 6 & 7 & 6.3 & Neg. & .58 & $.50 \mathrm{NS}$ \\
& 7 & 6 & 6 & & & & \\
CRPG/MWPS & 22 & 20 & - & 20 & $<.0$ & 1.0 & 1 . OONS \\
& 20 & 20 & - & & & & \\
USGSR/OES & 5 & 6 & 6 & 5.7 & Neg. & .6 & $.50 \mathrm{NS}$ \\
& 6 & 6 & 5 & & & & \\
\#ETH/XRP & 9 & 5 & 11 & 8 & - & - & - \\
*ETH/XRF & 19 & 19 & 19 & 19 & - & - & - \\
Kjel1/ICPS & 13 & 8 & 7 & 9 & - & - & - \\
/INAA & $<10$ & 20 & $<10$ & $<10$ & - & - & - \\
\hline
\end{tabular}

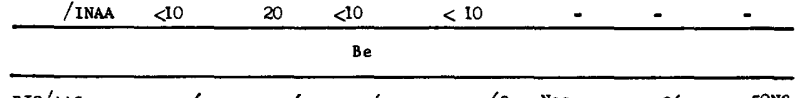

\begin{tabular}{lllllllll}
\hline BIO/AAS & .6 & .6 & .6 & .63 & Neg. & .06 & $.50 \mathrm{NS}$ \\
& .6 & .7 & .7 & & & & \\
GCL/AAS & .6 & .6 & .4 & .50 & .08 & .08 & $3.00 \mathrm{NS}$ \\
& .4 & .6 & .4 & & & & \\
\hline
\end{tabular}

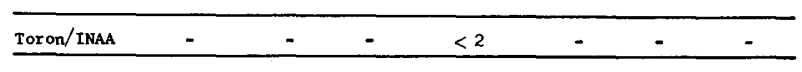

\begin{tabular}{llllllll}
\multicolumn{8}{c}{$\mathrm{Cd*}$} \\
\hline *B10/AAS & 97 & 96 & 95 & 97.8 & 1.2 & 5.7 & $1.09 \mathrm{NS}$
\end{tabular}

$\begin{array}{lrrrrrrr} & 103 & 90 & 106 & & & & \\ \text { *WAIT/IDMS } & 264 & 169 & - & 166.8 & \text { Neg. } & 76 & .47 \mathrm{NS}\end{array}$

*GCL/AAS $\quad \begin{array}{rrrrrrr}.80 & .56 & .64 & .61 & \text { Neg. } & .15 & .24 \mathrm{NS}\end{array}$

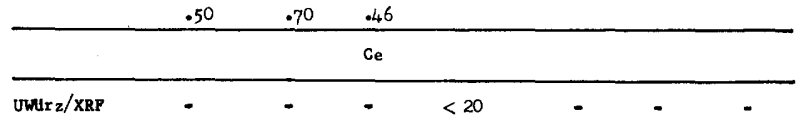

$\begin{array}{lccccccc}\mathrm{UWWI} z / \mathrm{XRP} & - & - & - & <20 & - & - & - \\ \mathrm{B} 1 \mathrm{rm} / \mathrm{XRP} & 2.2 & 4.6 & - & 3.6 & \text { Neg. } & 1.59 & .02 \mathrm{NS}\end{array}$

$\begin{array}{lcccccc} & 3.1 & 4.2 & - & & & \\ & 6.3 & 3.3 & - & & & \\ \text { Nott } / \mathrm{XRF} & - & - & - & <4 & -\end{array}$

$\begin{array}{llllllll}* \text { Toron/ INAA } & 4.2 & 5.3 & 5.7 & 5.41 & \text { Neg. } & .70 & .21 \mathrm{NS}\end{array}$

$\begin{array}{lllllllll} & 5.3 & 5.2 & 5.1 & & & & & \\ & 6.2 & 6.3 & 5.4 & & & & \\ * \text { *HMI/INAA-W } & 1.0 & 1.9 & - & 1.2 & .12 & .40 & 1.29 \mathrm{NS} \\ & 1.1 & 1.5 & - & & & & \end{array}$

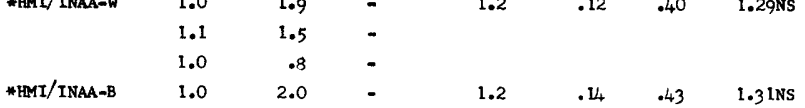

$\begin{array}{lccccccc}\text { \#HMI/INAA-B } & 1.0 & 2.0 & - & 1.2 & . \mathrm{L}_{4} & .43 & 1.31 \mathrm{NS} \\ & 1.1 & 1.5 & - & & & & \\ & 1.0 & .8 & - & & & & \\ \text { LASL/INAA-1 } & 10 & 15 & - & 13.0 & 0 & 2.3 & *\end{array}$

$\begin{array}{llllllll}\text { LASL/INAA-1 } & 10 & 15 & - & 13.0 & 0 & 2.3 & * \\ & 15 & 13 & - & & & & \end{array}$

$\begin{array}{llllllll}\text { Open/INAa } & 1.6 & 1.5 & - & 2.08 & \text { Neg. } & .57 & .41 \mathrm{NS}\end{array}$

$\begin{array}{llllllll} & 2.2 & 3.0 & - & & & & \\ \text { USGSR/INA } & 2.0 & 2.2 & - & & & & \\ & 4 & 4 & 4 & 4 & - & - & - \\ \text { Tohok/IPAA } & 4 & 4 & 4 & & & & \\ & 2.2 & 1.7 & 2.0 & 1.9 & .2 & .1 & 8.17 \mathrm{NS}\end{array}$

$1 / \operatorname{ETH}$ ( V. Dietrich) reports $6.8 \mathrm{ppm}$ Ba by IDMS.

* Cd in ppb by BIO and WAIT but in ppm by GCL.

\begin{tabular}{|c|c|c|c|c|c|c|c|}
\hline \multirow[b]{3}{*}{ Ore./Meth. } & \multirow{3}{*}{$\begin{array}{l}\text { Berte } \\
1 \\
\end{array}$} & \multirow{2}{*}{\multicolumn{2}{|c|}{ Number }} & \multirow[b]{3}{*}{ Mean } & \multicolumn{3}{|c|}{ Standard Deviation } \\
\hline & & & & & \multicolumn{2}{|c|}{ Botale Brrox } & \multirow{2}{*}{$\begin{array}{c}\bar{F} \\
\text { ratio } \\
\end{array}$} \\
\hline & & 2 & 3 & & $2 d f$ & $3 \mathrm{df}$ & \\
\hline \multicolumn{8}{|c|}{ Ce (cont.) } \\
\hline \multirow[t]{2}{*}{ KJe11/INAA } & 2.02 & 2.10 & 2.13 & 2.08 & - & - & - \\
\hline & & & $\mathrm{Cl}$ & & & & \\
\hline \multirow[t]{3}{*}{ *BMI/SSMS } & 40.7 & 26.8 & - & 32.5 & 7.07 & 3.03 & 11.97 \\
\hline & 34.7 & 27.7 & - & & & & \\
\hline & 28.2 & - & - & & & & \\
\hline \multirow[t]{3}{*}{ Munich } & 86 & 90 & - & 82.7 &.$\$ 2$ & 5.5 & $1.06 \mathrm{NS}$ \\
\hline & 81 & 85 & - & & & & \\
\hline & 74 & 80 & - & & & & \\
\hline
\end{tabular}

\begin{tabular}{llllllll}
\multicolumn{8}{c}{ Co } \\
\hline GSF/OES & 48 & 49 & 51 & 49 & 1.4 & .58 & 13.50
\end{tabular}

$\mathrm{GSC} / \mathrm{N}$

BIO/AAS

Parma/AAS

WHOI/XRF

$51.4 \quad 50.0 \quad 51.3$

$\begin{array}{lll}50.5 & 51.7 & 50.3\end{array}$

$\begin{array}{lllllllll}\text { UWUrz } / \text { XRF } & 50 & 49 & 49 & 49.2 & .7 & .4 & 7.00 \text { NS }\end{array}$

*Liege/XRF

Nott $/$ XRF

50.40

50.47

$51.18 \quad 53.51$

$\begin{array}{lllcllll} & 51.18 & 53.51 & - & & & & \\ \text { *Toron/INAA } & 57.0 & 66.2 & 60.6 & 60.28 & \text { Neg. } & 3.57 & .55 \mathrm{NS}\end{array}$

$60.4 \quad 56.1 \quad 62.6$

$60.3 \quad 56.4 \quad 62.9$

*HMI/INAA-W

54.3

54.0

*HMI/INAA-B

52.8

54.6

NERF/INAA 53.0

53.0

LASL/INAA-1 54

open/INAA

$51.9 \quad 51.2$

57.2

$-$

55

$64 \quad \mathrm{Ne}$

$52 \quad$ Neg. $\quad 2.4 \quad$.46NS

$57 \cdot$

$2.38 \quad 1.78 \quad 6.37$ NS

$51.6 \quad 52.1$

$49.8 \quad 51.6$

USGSR/INAA 52.6

49.

*LASL/INAa 51.

49.7

49.9

$\begin{array}{llll}50.6 & \text { Neg. } & 1.9 & .31 \mathrm{NS}\end{array}$

$52.4 \quad$ Neg. 1.14 .8ONS

$53.2 \quad 52.6$

Tohok/IPAA

50

UInd/AAS

57

Exxon/DCPAS

GRPG/MWPS

$51.1 \quad 49.9$

$48.4 \quad 49.4$

USGSR/OES

79
43

*HMI/SSMS

51

ETH/XRF

Kjell/AAS

/ICPS

/INAA

48.9
52.6
51.7

58
52

47

5

47

48

$-$

-

75

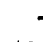

$49 \quad 48.2 \quad$ Neg. $\quad 3.6 \quad$.40NS

51

-

18.5 Neg. $4.1 \quad .51 \mathrm{NS}$ 
Table 3. Analytical data for USGS-BIR-1 (cont.)

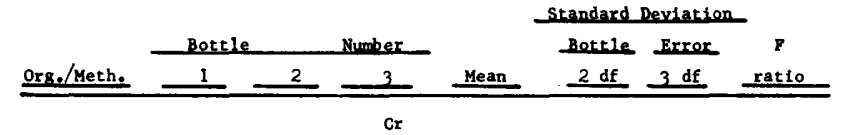

\begin{tabular}{llllllll}
\hline GSF/OES & 560 & 470 & 480 & 487 & Neg. & 49 & 0.13 NS
\end{tabular}

$\begin{array}{llllllll}\text { GSC } / \mathrm{N} & 410 & 380 & 400 & 392 & 21 & 12 & 6.78 \mathrm{NS}\end{array}$

$\begin{array}{llllllll} & 420 & 360 & 380 & & & & \\ \text { BIO/AAS } & 476 & 476 & 486 & 479 & 2.9 & 4.6 & 1.82 \mathrm{NS}\end{array}$

$\begin{array}{rrrrrrrr} & 476 & 476 & 486 & 479 & 2.9 & 4.6 & 1.82 N S \\ \text { Parma/AAS } & 485 & 473 & 480 & & & & \\ & 445 & 441 & - & 445.8 & \text { Neg. } & 5.4 & .28 N S\end{array}$

$\begin{array}{llll}450 & 49 & - & \\ 439 & 451 & - & 4408\end{array}$

$\begin{array}{llllllll}\text { WHOI/XRF } & 309.1 & 309.9 & 319.8 & 311 . \mathrm{t} & \text { Neg. } & 4.5 & .76 \mathrm{NS}\end{array}$

$\begin{array}{llllllll}\text { UWUr z } / \text { XRF } & 420 & 420 & 410 & 417 & 6.2 & 2.2 & 17.20\end{array}$

$B i \mathrm{rm} / \mathrm{XRF} \quad 345.8 \quad 347.9 \quad-\quad 341.8 \quad \mathrm{Neg} . \quad 5.02 \quad .10 \mathrm{NS}$

$\begin{array}{llllllll} & 335.5 & 338.9 & - & & & & \\ & 342.0 & 340.4 & - & & & & \\ \text { Nott } / \mathrm{XRF} & 378.37 & 374.26 & - & 372.67 & \text { Neg. } 10.02 & .29 \mathrm{NS}\end{array}$

$\begin{array}{llllllll} & 384.78 & 375.26 & - & & & & \\ & 361.49 & 361.84 & - & & & & \\ \text { *Toron/INAA } & 404 & 406 & 418 & 415.9 & 8.1 & 9.0 & 3.43 \mathrm{NS}\end{array}$

$432 \quad 400 \quad 426$

$\begin{array}{lll}422 & 409 \quad 426\end{array}$

$\begin{array}{lllllll}376 & 384 & - & 376 & 4.7 & 6.8 & 2.4 \text { NS } \\ 370 & 386 & - & & & & \end{array}$

*HMI/INAA-B $\quad 550 \quad 610$

$585 \quad 545$

$540 \quad 585$

NERE/INAA $\quad 378 \quad 386$

$\begin{array}{lll}382 & 381 \\ & 375 & 378\end{array}$

$\begin{array}{lll}375 & 378\end{array}$

$381 \quad 371$

USGSR/INAA 368.0 371.0 393.

$\begin{array}{llllllll} & 361.0 & 370.0 & 390.0 & & & & \\ \text { LASL/INAA } & 343 & 375 & - & 357 & \text { Neg. } & 12.6 & .42 \mathrm{NS}\end{array}$

$\begin{array}{llllllll} & 351 & 354 & - & & & & \\ & 368 & 353 & - & & & & \\ \text { Tohok/IPAA } & 342 & 335 & 338 & 338 & 4.5 & 2.4 & 8.03 \text { NS }\end{array}$

$\begin{array}{llllllll}\text { Tohok/IPAA } & 342 & 335 & 338 & 338 & 4.5 & 2.4 & 8.03 \mathrm{NS} \\ & 345 & 335 & 333 & & & & \\ \text { Exxon/DCPAS } & 408.3 & 436.2 & - & 412.4 & \text { Neg. } & 15.2 & .27 \mathrm{NS}\end{array}$

$\begin{array}{llll}421.4 & 407.2 & - \\ & 397.9 & 403.5 & -\end{array}$

$\begin{array}{llllllll}\text { CRPG/MWPS } & 400 & 403 & - & 404 & \text { Neg. } & 3.6 & .02 N S \\ & 407 & 405 & - & & & & \\ \text { USGSR/OES } & 440 & 440 & 40 & 427 & \text { Neg. } & 23\end{array}$

$\begin{array}{llllllll}\text { USGSR/OES } & 440 & 440 & 440 & 427 & \text { Neg. } 23 & .50 N S\end{array}$

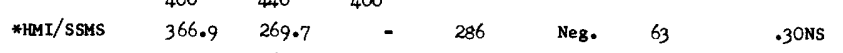

\begin{tabular}{clcccccc} 
& 240.1 & 267.3 & - & & & & \\
& 308.6 & - & - & & - & - \\
ETH/AAS & 380 & 375 & - & 378 & - & - & - \\
$/$ XRF & 374 & 373 & 387 & 378 & - & - & - \\
Kje11/IGPS & 404 & 408 & 406 & 406 & - & - & - \\
/ INAA & 481 & 453 & 481 & 473 & - & - & - \\
BMNH/XRP & 341 & 338 & 343 & 340.2 & 2.9 & 2.2 & $4.59 \mathrm{NS}$ \\
& 336 & 338 & 345 & & & & \\
\hline
\end{tabular}

\begin{tabular}{llllllll}
\hline \multicolumn{7}{c}{ Gs } \\
\hline GSC/M & .5 & .6 & .4 & .4 & Neg. & .26 & .88 NS
\end{tabular}

$\begin{array}{cccccccc} & .6 & .1 & .0 & & & & \\ \text { Toron/ INAA } & - & - & - & <1.4 & - & - & - \\ \text { *LASL/INAA-1 } & .4 & .4 & - & .5 & \text { Neg. } & .22 & .14 \text { NS } \\ & .7 & .8 & - & & & & \end{array}$

USGSR/INAA

*HMYI/SSAS

-3

(1)

$1.51 \quad 3.04$

$2.97 \quad 1.90 \quad-$

$2.92 \quad-\quad-$

Kjell/INAA

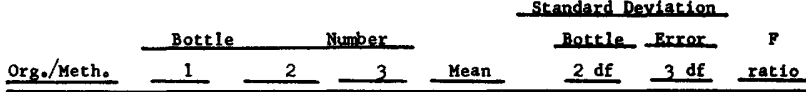

$\longrightarrow$ -

\begin{tabular}{llllllll}
\hline GSF/OES & 140 & 120 & 110 & 126.7 & Neg. & 15 & $0.07 N S$ \\
& 120 & 130 & 140 & & & & \\
GSC/N & 120 & 125 & 120 & 125.8 & Neg. & 6.8 & $.36 \mathrm{NS}$ \\
& 135 & 130 & 125 & & & &
\end{tabular}

$\begin{array}{lrrrrrrr} & 122 & 122 & 122 & & & & \\ \text { Parma/AAS } & 115 & 122 & - & 119.8 & \text { Neg. } & 6.6 & .19 N S\end{array}$

$\begin{array}{llll}128 & 111 & - & - \\ 120 & 123 & -\end{array}$

$\begin{array}{llllllll}\mathrm{WHOI} / \mathrm{XRF} & 126.1 & 122.4 & 128.9 & 126.4 & 2.6 & 2.1 & 4.17 \mathrm{NS}\end{array}$

$\begin{array}{lllllllll}\text { UWUIz/XRF } & 125 & 130 & 126 & 128 & 3.1 & 2.3 & 4.62 \mathrm{NS}\end{array}$

$\begin{array}{llllllll}\text { Nott } / \text { XRF } & 121.34 & 124.57 & - & 121.91 & \text { Neg. } & 2.32 & \text {.41NS }\end{array}$

$\begin{array}{llllllll} & 124.30 & 119.10 & - & & & & \\ & 121.92 & 120.24 & - & & & & \\ \text { Curie/XRF } & 115.60 & 116.24 & - & 116.96 & \text { Neg. } & 1.6 & \text {.IONS }\end{array}$

$\begin{array}{lllllllll} & 119.52 & 117.63 & - & & & & \\ & 116.38 & 116.39 & - & & & & \\ \text { UInd/ICPS } & 120 & 126 & 126 & 122.5 & \text { Neg. } & 4.2 & \text {.08NS }\end{array}$

$\begin{array}{llllllll} & 124 & 121 & 118 & & & & \\ \text { Exxon/DCPAS } & 131.6 & 131.8 & - & 131.0 & \text { Neg. } & 3.3 & .74 \text { NS }\end{array}$

$\begin{array}{llll}130.7 & 128.3 & - \\ 127.3 & 136.5 & -\end{array}$

$\begin{array}{llllllll}\text { CRPG/MWPS } & 130 & 121 & - & 125 & 5.2 & 1.1 & 45.00\end{array}$

$\begin{array}{llllllll}\text { USGSR/OES } & 128 & 122 & 0^{-} & & & & \\ & 150 & 150 & 160^{\circ} & 153 & 5.8 & 0\end{array}$

$\begin{array}{llllllll}* \text { HMI/SSMS } & 150 & 150 & 160 & & & & \\ & 39.6 & 32.9 & - & 33.3 & \text { Neg. } & 5.1 & .25 \mathrm{NS}\end{array}$

$\begin{array}{lccccccc} & 29.5 & 31.1 & - & & & & \\ & 44.8 & - & - & & & & \\ \text { USGSR/AAS } & 132 & 130 & 130 & 131.0 & 1.2 & .6 & 10.50\end{array}$

$\begin{array}{lrrrrrrr} & 133 & 131 & 130 & & & & \\ \text { ETH/AAS } & 125 & 125 & - & 125 & - & -\end{array}$

$\begin{array}{lllll}/ \mathrm{XRF} & 125 & 126 & 127 & 126\end{array}$

KJe11/AAS $\quad 96 \quad 97 \quad 95 \quad 96$

/ICPS 191

\begin{tabular}{|c|c|c|c|c|c|c|}
\hline /ICPS & 131 & 130 & 132 & 131 & - & - \\
\hline /OES & 60 & 87 & 85 & 77 & - & - \\
\hline
\end{tabular}

\begin{tabular}{llllllll}
\hline \multicolumn{7}{c}{ Dy } \\
\hline *Toron/ INAA & 1.88 & 1.81 & 2.25 & 2.32 & Neg. & .43 & .27 NS \\
& 2.75 & 1.99 & 2.55 & & & &
\end{tabular}

$\begin{array}{llllllll} & 2.15 & 2.87 & 2.59 & & & & \\ \text { NERF/INAA } & 2.8 & 1.9 & 2.5 & 3.45 & \text { Neg. } & 1.58 & \text {.4ONS }\end{array}$

$\begin{array}{llllllll} & 4.3 & 3.5 & 5.7 & & & & \\ * \text { Genev/INAA } & 5.67 & 4.34 & 4.80 & 5.28 & \text { Neg. } & 1.03 & .34 \mathrm{NS}\end{array}$

$\begin{array}{llllllll}4.60 & 5.57 & 6.73 & \end{array}$

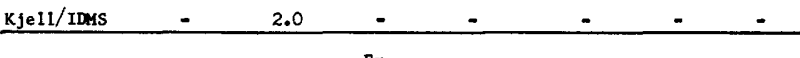

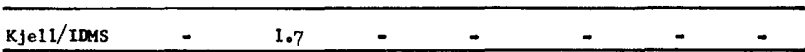

\begin{tabular}{lllllllll}
\multicolumn{8}{c}{ Eu } \\
\hline *Toron/ INAA & .70 & .67 & .68 & .68 & .01 & .06 & $1.10 \mathrm{NS}$
\end{tabular}

$\begin{array}{lll}.68 & .71 & .65\end{array}$

$\begin{array}{lllllllll} & .54 & .76 & .73 & & & & \\ \text { Toron/ INAA } & .48 & .65 & .60 & .57 & \text { Neg. } & .068 & .15 \mathrm{NS}\end{array}$

$\begin{array}{lllllllll} & .65 & .50 & .57 & & & & & \\ & .53 & .60 & .55 & & & & & \\ * \text { HMI/INAA-W } & .565 & .560 & - & .529 & \text { Neg. } & .039 & .24 \text { NS }\end{array}$

$.468 \quad .541 \quad-$

$.530 \quad .509 \quad-$

*HMI/INAA-B $\quad .50 \quad .55 \quad-$

$\begin{array}{lllllll}.50 & .55 & - & .498 & .027 & .024 & 4.97 \mathrm{NS} \\ .46 & .50 & - & & & & \\ .47 & .51 & - & & & & \end{array}$

$\begin{array}{llllllll}\text { NERE } / \text { INAA } & .53 & .52 & .49 & .56 & \text { Neg. } & .074 & .09 N S\end{array}$ 
Table 3. Analytical data for USGS-BIR-I (cont.)

\begin{tabular}{|c|c|c|c|c|c|c|c|}
\hline \multirow[b]{3}{*}{ Org./Meth. } & \multirow{3}{*}{$\begin{array}{l}\text { Betile } \\
1\end{array}$} & \multirow{2}{*}{\multicolumn{2}{|c|}{ Number }} & \multirow[b]{3}{*}{ Mean } & \multicolumn{2}{|c|}{ ard Deviation } & \multirow{3}{*}{$\begin{array}{r}\mathbf{P} \\
\text { ratio } \\
\end{array}$} \\
\hline & & & & & Bottle & Error & \\
\hline & & 2 & 3 & & $2 \mathrm{df}$ & $3 \mathrm{df}$ & \\
\hline \multicolumn{8}{|c|}{ Eu (cont.) } \\
\hline \multirow[t]{3}{*}{ LASL/INAA-1 } & 0.48 & 0.43 & - & 0.52 & Neg. & 0.07 & $0.24 \mathrm{NS}$ \\
\hline & .62 & .52 & - & & & & \\
\hline & .51 & .56 & - & & & & \\
\hline \multirow[t]{3}{*}{ Open/INAA } & .54 & .56 & - & .545 & Neg. & .012 & $.12 \mathrm{NS}$ \\
\hline & .55 & .53 & - & & & & \\
\hline & .55 & .54 & - & & & & \\
\hline \multirow[t]{2}{*}{ USGSR/INAA } & .52 & .49 & .51 & .502 & Neg. & $.0 u_{4}$ & $.36 \mathrm{NS}$ \\
\hline & .49 & .50 & .50 & & & & \\
\hline \multirow[t]{3}{*}{ LASL/INAA } & .72 & .82 & - & .83 & Neg. & .12 & $.08 \mathrm{NS}$ \\
\hline & .76 & .74 & - & & & & \\
\hline & .97 & .93 & - & & & & \\
\hline \multirow[t]{3}{*}{ Genev/INAA } & .86 & .83 & .90 & .85 & .05 & .03 & $5.55 \mathrm{NS}$ \\
\hline & .80 & .78 & .92 & & & & \\
\hline & - & .75 & .65 & & & & \\
\hline \multirow[t]{3}{*}{ *HMI/SSMS } & .63 & .39 & - & .51 & .22 & .05 & 35.12 \\
\hline & .71 & .32 & - & & & & \\
\hline & 1.15 & - & - & & & & \\
\hline Kjel1/IDMS & - & .6 & - & - & - & - & - \\
\hline
\end{tabular}

\begin{tabular}{cccccccc} 
/INAA & .52 & .50 & .52 & .51 & - & - & - \\
\hline \multirow{7}{*}{ *HMI/SSMS } & 40.5 & 32.6 & - & 36.8 & 1.54 & 3.0 & $1.53 \mathrm{NS}$ \\
& 36.8 & 37.3 & - & & & & \\
\multirow{4}{*}{ Munich } & 55.7 & - & - & & & & \\
& 55 & 53 & - & 58.8 & Neg. & 4.8 & $.36 \mathrm{NS}$ \\
& 65 & 58 & - & & & & \\
& 60 & 62 & - & & & & \\
\hline \multicolumn{7}{c}{ Ga }
\end{tabular}

\begin{tabular}{|c|c|c|c|c|c|c|c|}
\hline \multirow[t]{2}{*}{ GSF/OES } & 18 & 19 & 20 & 16 & Neg. & $4 \cdot 4$ & $.34 \mathrm{NS}$ \\
\hline & 10 & 14 & 15 & & & & \\
\hline \multirow[t]{2}{*}{$\mathrm{UWUI}_{\mathrm{Z}} / \mathrm{XRF}$} & $\mathrm{H}_{4}$ & 13 & 12 & 13.5 & Neg. & .9 & $.60 \mathrm{NS}$ \\
\hline & 14 & $\mathrm{u}_{4}$ & 14 & & & & \\
\hline \multirow[t]{3}{*}{$\mathrm{Birm} / \mathrm{XRF}$} & 14.7 & 15.7 & - & 15.0 & .61 & .43 & $7.13 \mathrm{NS}$ \\
\hline & 14.5 & 15.9 & - & & & & \\
\hline & 14.4 & 14.8 & - & & & & \\
\hline \multirow[t]{3}{*}{ Nott/XRF } & 14.64 & 14.46 & - & 15.13 & .30 & .77 & $1.45 \mathrm{NS}$ \\
\hline & 15.38 & 15.86 & - & & & & \\
\hline & 14.22 & 16.20 & - & & & & \\
\hline \multirow[t]{3}{*}{ Curie/XRF } & 15.61 & 15.57 & - & $15 \cdot 31$ & Neg. & 1.5 & $.2 \mathrm{INS}$ \\
\hline & 14.15 & 16.26 & - & & & & \\
\hline & 17.02 & 13.27 & - & & & & \\
\hline \multirow[t]{2}{*}{ USGSR/OES } & 19 & 19 & 20 & 19.8 & .8 & .9 & $2.60 \mathrm{NS}$ \\
\hline & 19 & 20 & 22 & & & & \\
\hline \multirow[t]{3}{*}{ *FMI/SSMS } & 9.0 & 6.6 & - & 7.0 & $\mathrm{Neg}$ & 1.5 & $.48 \mathrm{NS}$ \\
\hline & 6.0 & 6.3 & - & & & & \\
\hline & 10.7 & - & - & & & & \\
\hline $\operatorname{ETH} / \mathrm{XRF}$ & 19 & 19 & 19 & 19 & - & - & - \\
\hline
\end{tabular}

\begin{tabular}{lccccccc}
\multicolumn{7}{c}{ Gd } \\
\hline Open/INAA & 2.6 & 2.2 & - & 2.18 & .18 & .21 & $3.1_{4} \mathrm{NS}$ \\
& 2.3 & 2.0 & - & & & & \\
& 2.1 & 1.89 & - & & & & \\
USGSR/INAA & 1.5 & 1.3 & $<3.0$ & 1.6 & - & - & - \\
& 2.2 & 1.6 & 1.4 & & & & \\
KJel1/IDMS & - & - & - & 3.0 & - & - & - \\
\hline
\end{tabular}

\begin{tabular}{lllllllll}
\multicolumn{7}{c}{ Hf } \\
*HMI/ INAA-W & .61 & .63 & - & .595 & Neg. & .036 &. . I2NS \\
& .63 & .56 & - & & & & &
\end{tabular}

\begin{tabular}{|c|c|c|c|c|c|c|c|}
\hline & .56 & .58 & - & & & & \\
\hline \multirow[t]{3}{*}{ * HMI/INAA-B } & .60 & .59 & - & .543 & Neg. & .056 & $.02 \mathrm{NS}$ \\
\hline & .56 & .49 & - & & & & \\
\hline & .48 & .54 & - & & & & \\
\hline \multirow[t]{2}{*}{ NERF/INAA } & .60 & - & .57 & .56 & - & - & - \\
\hline & .50 & .58 & - & & & & \\
\hline \multirow[t]{3}{*}{ LASL/INAA-I } & .78 & .90 & - & .82 & Neg. & .09 & $.12 \mathrm{NS}$ \\
\hline & .92 & .86 & - & & & & \\
\hline & .72 & .74 & - & & & & \\
\hline
\end{tabular}

\section{Three USGS Mafic Rock Reference Samples}

\begin{tabular}{|c|c|c|c|c|c|c|c|}
\hline \multirow{3}{*}{ Ores/Methe } & \multirow{3}{*}{$\frac{\text { Bottile }}{1}$} & \multirow{2}{*}{\multicolumn{2}{|c|}{ Number }} & \multicolumn{3}{|c|}{ Standard Deviation } & \multirow{3}{*}{$\begin{array}{r}\mathbf{F} \\
\text { ratio } \\
\end{array}$} \\
\hline & & & & \multirow[b]{2}{*}{ Mean } & \multirow{2}{*}{$\frac{\text { Bottle }}{2 \text { df }}$} & \multirow{2}{*}{$\frac{\text { Error }}{3 \mathrm{df}}$} & \\
\hline & & 2 & 3 & & & & \\
\hline \multicolumn{8}{|c|}{ He (cont.) } \\
\hline \multirow[t]{3}{*}{ Open/INAa } & 0.58 & 0.56 & - & 0.603 & Neg. & 0.118 & $0.94 \mathrm{NS}$ \\
\hline & .84 & .56 & - & & & & \\
\hline & .53 & .55 & - & & & & \\
\hline USGSR/INAA & .8 & .7 & .6 & .66 & - & - & - \\
\hline & .6 & .6 & $<.8$ & & & & \\
\hline Kjel I/INAA & .6 & .8 & .5 & .6 & - & $=$ & - \\
\hline & & & Hо & & & & \\
\hline Genev/INAA & 1.93 & 1.92 & 1.85 & 1.91 & Neg. & .08 & $.63 \mathrm{NS}$ \\
\hline & 1.80 & 1.99 & 1.98 & & & & \\
\hline & - & 1.91 & 1.88 & & & & \\
\hline & & & La & & & & \\
\hline UWUI $z$ / XRF & 4 & 3 & 3 & 3.5 & .4 & .4 & $3.00 \mathrm{NS}$ \\
\hline & 4 & 4 & 3 & & & & \\
\hline Birm/XRF & 2.3 & 1.3 & - & 1.7 & Neg. & .65 &. $\mathrm{O}_{4} \mathrm{NS}$ \\
\hline & 1.9 & 1.8 & - & & & & \\
\hline & .7 & 2.1 & - & & & & \\
\hline Nott/XRF & 4.56 & 2.58 & - & 3.40 & .73 & .63 & $5.03 \mathrm{NS}$ \\
\hline & 4.23 & 3.36 & - & & & & \\
\hline & 3.12 & 2.52 & - & & & & \\
\hline *HMI/INAA-W & .57 & .54 & - & .518 & Neg. & .074 & $.03 \mathrm{NS}$ \\
\hline & .45 & .58 & - & & & & \\
\hline & .55 & .42 & - & & & & \\
\hline *HMI/INAA-B & .64 & .61 & - & .612 & Neg. & .038 & $.28 \mathrm{NS}$ \\
\hline & .60 & .65 & - & & & & \\
\hline & .62 & .55 & - & & & & \\
\hline *NERF/INAA & - & .72 & .82 & .74 & .083 & .043 & $8.56 \mathrm{NS}$ \\
\hline & - & .64 & .79 & & & & \\
\hline Open/INAA & .8 & 1.4 & - & 1.0 & Neg. & .40 &.$\alpha_{4} \mathrm{NS}$ \\
\hline & 1.5 & .6 & - & & & & \\
\hline & .8 & .9 & - & & & & \\
\hline USGSR/INAA & 1 & 1 & 1 & 1 & - & - & - \\
\hline & 1 & I & 1 & & & & \\
\hline Genev/INAA & .60 & .78 & .68 & .67 & Neg. & .12 & $.44 \mathrm{NS}$ \\
\hline & .80 & .62 & .52 & & & & \\
\hline & - & .57 & .61 & & & & \\
\hline Exxon/DCPAS & 5.0 & 7.2 & - & 6.0 & Neg. & .80 & $.84 \mathrm{NS}$ \\
\hline & 6.5 & 5.5 & - & & & & \\
\hline & 5.7 & 6.3 & - & & & & \\
\hline Kjel1/INAA & .78 & .72 & .75 & .75 & $=$ & - & $=$ \\
\hline & & & $\mathbf{L i}$ & & & & \\
\hline $\mathrm{GSC} / \mathrm{M}$ & 4 & 4 & 4 & 3.8 & $<.000$ & .4 & $1.00 \mathrm{NS}$ \\
\hline & 4 & 4 & 3 & & & & \\
\hline B IO/AAS & 3.9 & 3.8 & 3.9 & 3.9 & .03 & .07 & $1.33 \mathrm{NS}$ \\
\hline & 4.0 & 3.9 & 3.8 & & & & \\
\hline Parma/AAS & 3.4 & 3.3 & - & 3.35 & .04 & .10 & $1.50 \mathrm{NS}$ \\
\hline & 3.3 & 3.4 & - & & & & \\
\hline & 3.2 & 3.5 & - & & & & \\
\hline UWUIXz/XRP & 6 & 6 & 5 & 5.8 & 1.0 & .7 & $5.30 \mathrm{NS}$ \\
\hline & 7 & 7 & 4 & & & & \\
\hline USGSD/AAS & 3 & 3 & 3 & 3 & - & - & - \\
\hline & 3 & 3 & 3 & & & & \\
\hline USGSR/AAS & 3.5 & 3.3 & 3.5 & 3.40 & .14 & .15 & $2.78 \mathrm{NS}$ \\
\hline & 3.7 & 3.2 & 3.2 & & & & \\
\hline & & & Lu & & & & \\
\hline Toron/INAA & .25 & .27 & .18 & .208 & Neg. & .048 & $.50 \mathrm{NS}$ \\
\hline & .27 & .18 & .19 & & & & \\
\hline & .17 & .15 & .21 & & & & \\
\hline *HMI/INAA-W & .27 & .34 & - & .302 & Neg. & .034 & $.13 \mathrm{NS}$ \\
\hline & .34 & .29 & - & & & & \\
\hline & .28 & .29 & - & & & & \\
\hline *HMI/INAA-B & .26 & .31 & - & .292 & Neg. & .028 & $.02 \mathrm{NS}$ \\
\hline & .33 & .29 & - & & & & \\
\hline & .28 & .28 & - & & & & \\
\hline Open/INAA & .26 & .27 & - & .27 & .19 & .047 & $1.50 \mathrm{NS}$ \\
\hline & .36 & .21 & - & & & & \\
\hline & .26 & .26 & & & & & \\
\hline
\end{tabular}


Table 3. Analytical data for USGS-BIR-1 (cont.)

\begin{tabular}{|c|c|c|c|c|c|c|c|}
\hline \multirow[b]{3}{*}{ Org./Meth. } & \multirow{2}{*}{\multicolumn{3}{|c|}{ Number }} & \multirow[b]{3}{*}{ Mean } & \multicolumn{2}{|c|}{ Standard Deviation } & \multirow{3}{*}{$\begin{array}{c}F \\
\text { ratio } \\
\end{array}$} \\
\hline & & & & & \multirow{2}{*}{$\begin{array}{l}\text { Bottle } \\
2 \mathrm{df}\end{array}$} & \multirow{2}{*}{$\frac{\text { Exror }}{3 \mathrm{df}}$} & \\
\hline & 1 & 2 & 3 & & & & \\
\hline \multicolumn{8}{|c|}{ Lu (cont.) } \\
\hline \multirow[t]{2}{*}{ USGSR/INAA } & 0.27 & 0.25 & 0.27 & 0.26 & Neg. & 0.01 & $0.50 \mathrm{NS}$ \\
\hline & .25 & .26 & .26 & & & & \\
\hline \multirow[t]{3}{*}{ Genev/INAA } & .77 & .66 & .54 & .65 & Neg. & .16 & $.07 \mathrm{NS}$ \\
\hline & .47 & .70 & .77 & & & & \\
\hline & - & .66 & .66 & & & & \\
\hline \multirow[t]{2}{*}{ K je11/INAA } & .27 & .27 & .28 & .27 & - & - & - \\
\hline & & & Mn & & & & \\
\hline \multirow[t]{2}{*}{ B IO/AAS } & 1300 & 1305 & 1300 & 1290 & Neg. & 14 & $.43 \mathrm{NS}$ \\
\hline & 1290 & 1290 & 1270 & & & & \\
\hline \multirow[t]{2}{*}{ NERF/INAA } & 1525 & 1470 & 1575 & 1573 & 13 & 73 & $1.06 \mathrm{NS}$ \\
\hline & 1590 & 1590 & 1690 & & & & \\
\hline \multirow[t]{3}{*}{ LASL/INAA-I } & 1,00 & 1410 & - & 1397 & 1.7 & 13 & $6.40 \mathrm{NS}$ \\
\hline & 1370 & 1420 & - & & & & \\
\hline & 1380 & 1,00 & - & & & & \\
\hline \multirow[t]{2}{*}{ USGSR/OES } & 1,00 & 1500 & 1000 & 1200 & Neg. & 252 & $.24 \mathrm{NS}$ \\
\hline & 1100 & 1000 & 1200 & & & & \\
\hline \multirow[t]{3}{*}{ *HMI/SSMS } & 1181.7 & 790.9 & - & 996 & 209 & 63 & 23.06 \\
\hline & 1092.8 & 379.8 & - & & & & \\
\hline & 1099.8 & - & - & & & & \\
\hline
\end{tabular}

\begin{tabular}{lccccccc}
\multicolumn{7}{c}{ Mo } \\
\hline UInd/ICPS & 1.5 & 1.7 & 1.7 & 1.52 & Ne8. & .29 & $.26 \mathrm{NS}$ \\
& 1.7 & 1.1 & 1.4 & & & & \\
USGSR/Spph & .04 & .04 & .04 & .037 & $<.000$ & .008 & $1.00 \mathrm{NS}$ \\
& .02 & .04 & .04 & & & & \\
KjeII/INAA & - & - & - & $<.5$ & - & - & - \\
\hline
\end{tabular}

\begin{tabular}{|c|c|c|c|c|c|c|c|}
\hline \multirow[t]{2}{*}{ WHOI/XRF } & 2.7 & 2.4 & 2.8 & 2.3 & Neg. & .6 & $.78 \mathrm{NS}$ \\
\hline & 2.7 & 1.5 & 1.6 & & & & \\
\hline UWU r $z / X R F$ & - & - & - & $<3$ & - & - & - \\
\hline$B i r m / X R F$ & - & - & - & $<.4$ & - & - & - \\
\hline \multirow[t]{3}{*}{ Nott $/ \mathrm{XRF}$} & 1.58 & 2.65 & - & 2.66 & Neg. & 1.31 & $.20 \mathrm{NS}$ \\
\hline & 2.10 & 2.41 & - & & & & \\
\hline & 5.00 & 2.20 & - & & & & \\
\hline \multirow[t]{2}{*}{ Tohok/IPAA } & 1.8 & 1.6 & 1.6 & 1.65 & Neg. & .09 & $.60 \mathrm{NS}$ \\
\hline & 1.6 & 1.7 & 1.6 & & & & \\
\hline \multirow[t]{2}{*}{ USGSR/Spph } & 2.2 & 2.0 & 2.5 & 2.45 & .29 & .34 & $2.47 \mathrm{NS}$ \\
\hline & 2.5 & 2.2 & 3.2 & & & & \\
\hline ETH/XRF & 4 & 3 & 4 & 4 & - & - & - \\
\hline
\end{tabular}

\begin{tabular}{lccccccc}
\multicolumn{7}{c}{ Nd } \\
\hline Birm/XRF & 3.3 & 3.4 & - & 3.5 & Neg. & .22 & $.57 \mathrm{NS}$ \\
& 3.2 & 3.5 & - & & & & \\
& 3.7 & 3.7 & - & & & & \\
Open/INAA & 2.5 & 2.6 & - & 2.32 & .22 & .48 & $1.61 \mathrm{NS}$ \\
& 1.3 & 2.7 & - & & & & \\
& 2.4 & 2.4 & - & & & & \\
USGSR/INAA & - & - & - & $<4$ & - & - & - \\
ETH/XRF & 2 & 0 & 3 & 2 & - & - & - \\
KJel1/IDMS & - & - & - & 2.6 & - & - & - \\
\hline
\end{tabular}

\begin{tabular}{llllllll}
\multicolumn{7}{c}{ N1 } \\
\hline GSF/OES & 220 & 230 & 230 & 223 & $<.00$ & 8.2 & $1.00 \mathrm{NS}$ \\
& 220 & 230 & 210 & & & & \\
BMNH/XRF & 135 & 120 & 115 & 131.7 & Neg. & 13.5 & $.64 \mathrm{NS}$ \\
& 145 & 130 & 145 & & & & \\
GSC/N & 175 & 175 & 160 & 172.5 & 6.8 & 8.4 & $2.29 \mathrm{NS}$ \\
& 190 & 165 & 170 & & & & \\
Parma/AAS & 177 & 176 & - & 177.7 & .58 & 1.29 & $1.60 \mathrm{NS}$ \\
& 180 & 178 & - & & & & \\
& 178 & 177 & - & & & & \\
WHOI/XRF & 157.8 & 158.2 & 158.2 & 157.7 & Neg. & .9 & $.39 \mathrm{NS}$ \\
& 158.5 & 156.7 & 156.7 & & & & \\
UWUIZ/XRF & 168 & 173 & 165 & 171 & 3.5 & 2.8 & $4.08 \mathrm{NS}$ \\
& 172 & 177 & 169 & & & & \\
Liege/XRF & 173 & 174 & - & 176.7 & Neg. & 6.0 & 46NS \\
& 181 & 186 & - & & & &
\end{tabular}

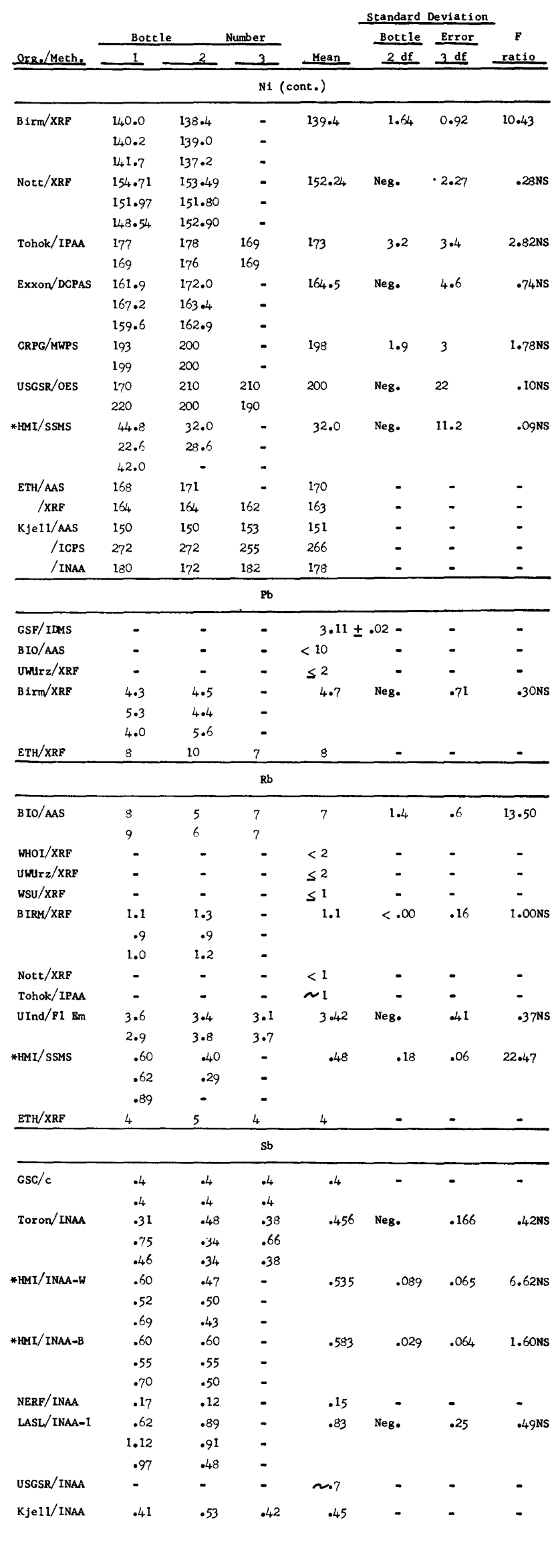


Table 3. Analytical data for USGS-BIR-1 (cont.)

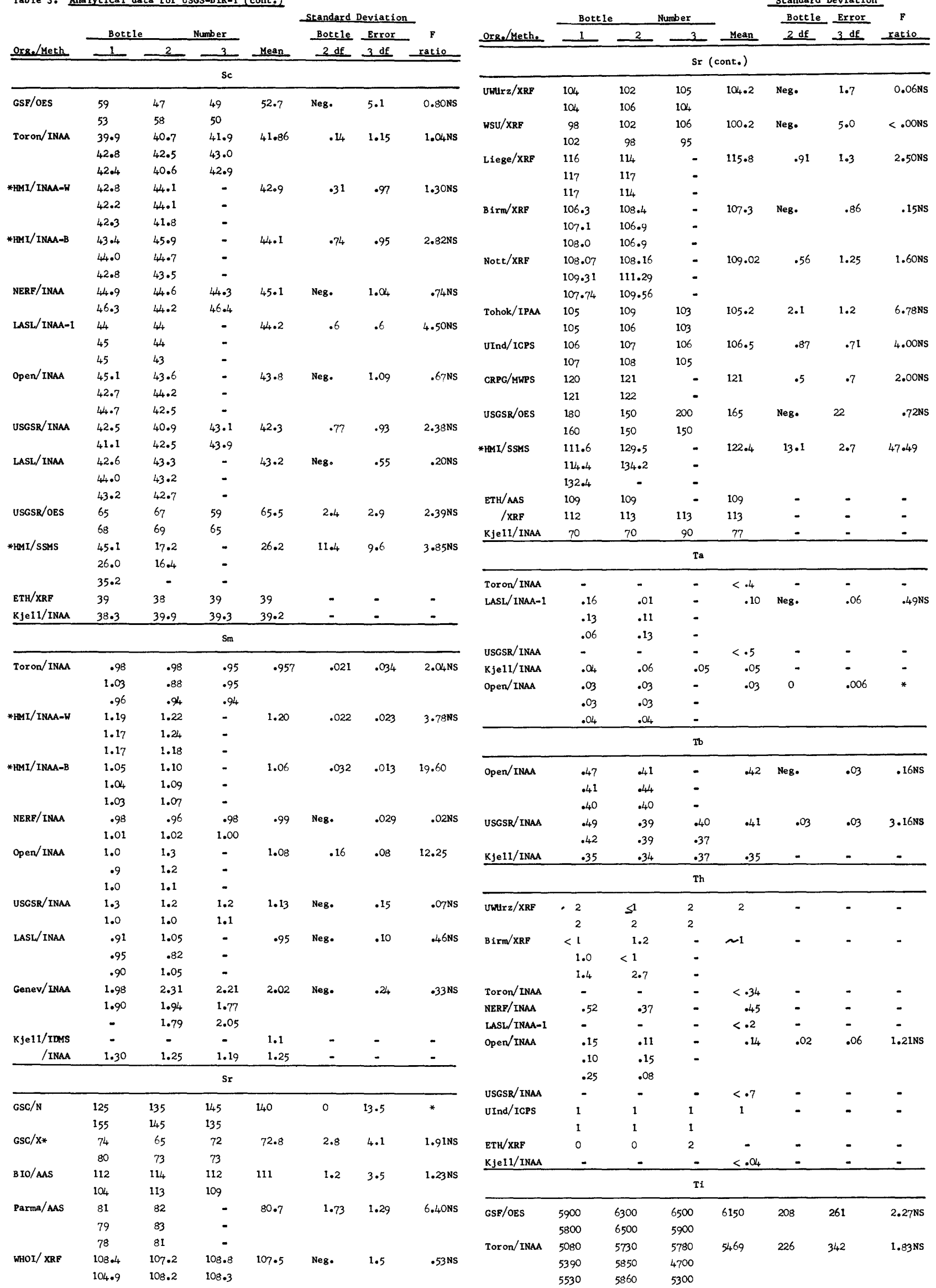


Table 3. Analytical data for USGS-B IR-1 (cont.)

\begin{tabular}{|c|c|c|c|c|c|c|c|}
\hline \multirow[b]{2}{*}{ Orea/Meche } & \multicolumn{2}{|c|}{ Bottle } & Number & \multirow[b]{2}{*}{ Mean } & \multicolumn{3}{|c|}{ Standard Deviation } \\
\hline & 1 & 2 & -3 & & $\begin{array}{l}\text { Botcle } \\
2 \mathrm{df} \\
\end{array}$ & $\begin{array}{l}\text { Error } \\
3 \text { df } \\
\end{array}$ & $\begin{array}{c}F \\
\text { ratie } \\
\end{array}$ \\
\hline \multicolumn{8}{|c|}{ Ti (cont.) } \\
\hline \multirow[t]{2}{*}{ USGSR/OES } & 3100 & 2900 & 3100 & 3100 & 58 & 115 & $1.50 \mathrm{Ns}$ \\
\hline & 3300 & 3100 & 3100 & & & & \\
\hline \multirow[t]{3}{*}{ *HMI/SSMS } & 3624 & 3135 & - & 3457 & Neg. & 48 & $.35 \mathrm{NS}$ \\
\hline & 3444 & 3624 & - & & & & \\
\hline & 3418 & - & - & & & & \\
\hline
\end{tabular}

\begin{tabular}{llllllll}
\hline USGSR/AAS & - & - & - & $<.10$ & - & - & - \\
\hline Open/INAA & .35 & .30 & - & .34 & Neg. & .043 & .15 NS \\
& .31 & .41 & - & & & & \\
& .35 & .34 & - & & & & \\
USGSR/INAA & .27 & .26 & .27 & .235 & Neg. & .04 & $.02 N S$ \\
& .20 & .20 & .21 & & & & \\
\hline
\end{tabular}

\begin{tabular}{|c|c|c|c|c|c|c|c|}
\hline & & & $\mathrm{u}$ & & & & \\
\hline $\mathrm{GSC} / \mathrm{U}$ & - & - & - & $<2$ & - & - & - \\
\hline \multirow[t]{2}{*}{ Toron/INAA } & 1.0 & 1.0 & 2.2 & 1.22 & .30 & .41 & $2.56 \mathrm{NS}$ \\
\hline & .7 & 1.5 & 1.7 & & & & \\
\hline
\end{tabular}

$\begin{array}{llllllll} & .8 & 1.2 & .9 & & & & \\ \text { LASL } / \text { DNAA }-1 & .01 & .03 & - & .03 & .02 & .01 & 10.12\end{array}$

\begin{tabular}{lccccccc} 
& .01 & .06 & - & & .02 & .01 & 10.12 \\
USGSR/INAA & -02 & .04 & - & & & & \\
ETH/XRF & 1 & - & - & $<.5$ & - & - & - \\
Kje11/INAA & - & - & 2 & 2 & - & - & - \\
\hline & & - & - & $<.05$ & - & - & - \\
\hline
\end{tabular}

\begin{tabular}{|c|c|c|c|c|c|c|c|}
\hline \multirow[t]{2}{*}{ GSF/OES } & 300 & 310 & 310 & 321.7 & Neg. & 22 & $.45 \mathrm{Ns}$ \\
\hline & 320 & 350 & 340 & & & & \\
\hline \multirow[t]{2}{*}{$\mathrm{Gsc} / \mathrm{N}$} & 320 & 310 & 300 & 323 & Neg. & 19 & $.32 \mathrm{NS}$ \\
\hline & 340 & 340 & 330 & & & & \\
\hline \multirow[t]{2}{*}{ BIO/AAS } & 300 & $3 \alpha_{4}$ & 299 & 302 & Neg. & 2.9 & $<.00 \mathrm{NS}$ \\
\hline & 304 & 301 & 304 & & & & \\
\hline \multirow[t]{3}{*}{ Parma/AAS-1 } & 280 & 290 & - & 304 & - & - & - \\
\hline & 315 & - & - & & & & \\
\hline & 330 & - & - & & & & \\
\hline \multirow[t]{3}{*}{ Pa rma/AAS-2 } & 319 & 341 & - & 332.5 & Neg. & 11.5 & $<.00 \mathrm{NS}$ \\
\hline & 345 & 322 & - & & & & \\
\hline & 334 & 334 & - & & & & \\
\hline \multirow[t]{2}{*}{ WHOI/XRF } & 301.1 & 302.2 & 300.9 & 300.5 & $\cdot 3$ & I. 5 & $1.03 \mathrm{NS}$ \\
\hline & 302.9 & 299.1 & 296.5 & & & & \\
\hline \multirow[t]{3}{*}{ Liege/XRF } & 294 & 289 & - & 290.8 & 3.7 & 5.7 & $2.27 \mathrm{NS}$ \\
\hline & 285 & 300 & - & & & & \\
\hline & 283 & 294 & - & & & & \\
\hline \multirow[t]{3}{*}{ Nott/XRF } & 259.00 & 254.92 & - & 255.56 & Neg. & 3.31 & $.35 \mathrm{NS}$ \\
\hline & 250.85 & 255.17 & - & & & & \\
\hline & 254.46 & 258.98 & - & & & & \\
\hline \multirow[t]{3}{*}{ Toron/INAA } & 338 & 332 & 318 & 330.2 & Neg. & $9 \cdot 9$ & $.08 \mathrm{NS}$ \\
\hline & 329 & 335 & 322 & & & & \\
\hline & 323 & 329 & 346 & & & & \\
\hline \multirow[t]{3}{*}{ LASL/INAA-1 } & 298 & 298 & - & 290.5 & 9.8 & 12.0 & $3.02 \mathrm{NS}$ \\
\hline & 272 & 290 & - & & & & \\
\hline & 276 & 309 & - & & & & \\
\hline \multirow[t]{2}{*}{ UInd/ICPS } & 309 & 305 & 305 & 306.8 & Neg. & 3.0 & $.36 \mathrm{NS}$ \\
\hline & 305 & 311 & 306 & & & & \\
\hline \multirow[t]{3}{*}{ Exxon/DCPAS } & 321.5 & 325.9 & - & 309.1 & Neg. & 14.9 & $<.00 \mathrm{NS}$ \\
\hline & $314 \cdot 3$ & 300.0 & - & & & & \\
\hline & 292.7 & 300.2 & - & & & & \\
\hline \multirow[t]{2}{*}{ CRPG/MWPS } & 304 & 301 & - & 304 & Neg. & 2.1 & $.53 \mathrm{NE}$ \\
\hline & 305 & 305 & - & & & & \\
\hline \multirow[t]{2}{*}{ USGSR/OES } & 480 & 480 & 530 & 528 & Neg. & 47 & .82NS \\
\hline & 520 & 570 & 590 & & & & \\
\hline \multirow[t]{3}{*}{ *HMI/SSMS } & 235.6 & 180.2 & - & 206.3 & $17 \cdot 9$ & 17.5 & $3.08 \mathrm{NS}$ \\
\hline & 207.8 & 201.6 & - & & & & \\
\hline & 172.3 & - & - & & & & \\
\hline $\mathrm{ETH} / \mathrm{XRF}$ & 317 & 315 & 320 & 317 & - & - & - \\
\hline
\end{tabular}

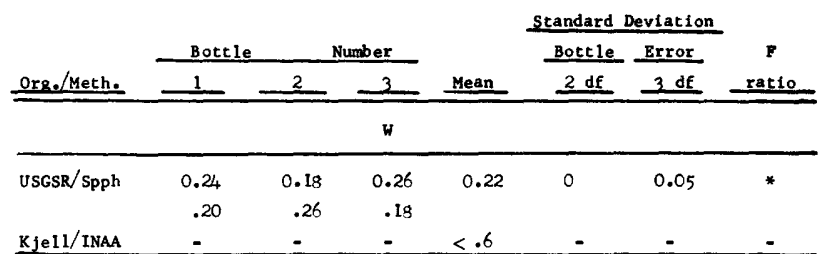

\begin{tabular}{|c|c|c|c|c|c|c|c|}
\hline Kjell/INAA & - & $=$ & - & $<.6$ & - & - & - \\
\hline & & & $\mathbf{Y}$ & & & & \\
\hline \multirow[t]{2}{*}{ GSF/OES } & 29 & 22 & 25 & 23.5 & Neg. & 5.0 & $.02 N S$ \\
\hline & 18 & 26 & 21 & & & & \\
\hline \multirow[t]{2}{*}{ WHOI/XRF } & 18.8 & 19.6 & 18.8 & $19 \cdot 3$ & .1 & .4 & $1.09 \mathrm{NS}$ \\
\hline & 19.3 & 19.6 & 19.6 & & & & \\
\hline \multirow[t]{2}{*}{ UWUrz/XRF } & 24 & 20 & 18 & 21.2 & 2.7 & .9 & 18.20 \\
\hline & 24 & 22 & 19 & & & & \\
\hline \multirow[t]{2}{*}{$\mathrm{WSU} / \mathrm{XRF}$} & 5 & 9 & 13 & $7 \cdot 2$ & .7 & 3.8 & $1.07 \mathrm{NS}$ \\
\hline & 3 & 9 & 4 & & & & \\
\hline \multirow[t]{3}{*}{ Liege/XRF } & 17 & 16 & - & 16.5 & Neg. & 1.2 & $.12 N S$ \\
\hline & 15 & 18 & - & & & & \\
\hline & 17 & 16 & - & & & & \\
\hline \multirow[t]{3}{*}{$\mathrm{Birm} / \mathrm{XRP}$} & 12.6 & $u .5$ & - & 14.6 & 0 & 1.38 & * \\
\hline & 16.2 & $15 \cdot 3$ & - & & & & \\
\hline & 14.9 & 13.9 & - & & & & \\
\hline \multirow[t]{3}{*}{ Nott $/ \mathrm{XRF}$} & 18.32 & $17 \cdot 37$ & - & $18 \cdot 36$ & Neg. & .72 & $.26 \mathrm{NS}$ \\
\hline & 18.35 & 19.23 & - & & & & \\
\hline & 17.97 & 18.95 & - & & & & \\
\hline \multirow[t]{2}{*}{ Tohok/IPAA } & 16 & 16 & 16 & 15.8 & $\cdot 3$ & .7 & $1.33 \mathrm{NS}$ \\
\hline & 17 & 15 & 15 & & & & \\
\hline \multirow[t]{2}{*}{ UInd/ICPS } & 16 & 16 & 16 & 16.2 & $<.00$ & .41 & $1.00 \mathrm{NS}$ \\
\hline & 16 & 17 & 16 & & & & \\
\hline \multirow[t]{2}{*}{ USGSR/OES } & 26 & 29 & 25 & 27.2 & .9 & 1.2 & $2.11 \mathrm{NS}$ \\
\hline & 28 & 28 & 27 & & & & \\
\hline \multirow[t]{3}{*}{ *HMI/SSMS } & 13.1 & 15.6 & - & 14.8 & 1.4 & .68 & 9.09 \\
\hline & $u_{4} .4$ & 16.0 & - & & & & \\
\hline & 16.3 & - & - & & & & \\
\hline $\mathrm{ETH} / \mathrm{XRF}$ & 18 & 18 & 19 & 18 & - & - & - \\
\hline
\end{tabular}

\begin{tabular}{lllllllll}
$\mathrm{ETH} / \mathrm{XRF}$ & 18 & 18 & 19 & 18 & - & - & - \\
\hline & & & & & \\
\hline
\end{tabular}

\begin{tabular}{llllllll}
\hline Toron/INAA & 1.6 & 1.9 & 1.2 & 1.63 & .16 & .22 & $2.57 \mathrm{NS}$ \\
& 1.6 & 1.5 & 1.5 & & & & \\
& 1.7 & 2.1 & 1.6 & & & & \\
*HMI/INAA-W & 1.72 & 1.58 & - & 1.63 & Neg. & .092 & $.56 \mathrm{NS}$ \\
& 1.53 & 1.73 & - & & & & \\
& 1.54 & 1.65 & - & & & & \\
*HMI/INAA-B & 1.53 & 1.60 & - & 1.55 & .024 & .025 & $3.69 \mathrm{NS}$ \\
& 1.55 & 1.57 & - & & & & \\
& 1.51 & 1.54 & - & & & & \\
NERF/INAA & 1.94 & 1.76 & 2.04 & 1.97 & .16 & .096 & $6.76 \mathrm{NS}$ \\
& 1.95 & 1.84 & 2.26 & & & & \\
Open/INAA & 1.73 & 1.68 & - & 1.695 & .011 & .040 & $1.23 \mathrm{NS}$ \\
& 1.76 & 1.67 & - & & & & \\
& 1.65 & 1.68 & - & & & & \\
USGSR/INAA & 1.6 & 1.6 & 1.7 & 1.6 & .08 & .06 & $4.50 \mathrm{NS}$ \\
& 1.5 & 1.5 & 1.7 & & & & \\
LASL/INAA & - & 1.47 & - & 1.75 & - & - & - \\
& 1.40 & 1.58 & - & & & & \\
& 1.86 & 2.15 & - & & & & \\
Genev/INAA & 2.63 & 2.24 & 2.13 & 2.24 & Neg. & .22 & $.70 \mathrm{NS}$ \\
& 2.09 & 2.30 & 2.07 & & & & \\
& - & 2.09 & 2.08 & & & &
\end{tabular}

\begin{tabular}{|c|c|c|c|c|c|c|c|}
\hline \multirow[t]{2}{*}{ USGSR/OES } & 3 & 3 & 3 & 3 & - & - & - \\
\hline & 3 & 3 & 3 & & & & \\
\hline \multirow{3}{*}{$\begin{array}{r}\text { Kjel1/IDMS } \\
\text { /INAA } \\
\end{array}$} & - & - & - & 3.5 & - & - & - \\
\hline & 1.56 & 1.58 & 1.62 & 1.59 & - & - & - \\
\hline & & & $2 n$ & & & & \\
\hline \multirow[t]{2}{*}{ BIO/AAS } & 72 & 71 & 70 & 71 & $\cdot 3$ & .6 & $1.40 \mathrm{NS}$ \\
\hline & 71 & 71 & 71 & & & & \\
\hline \multirow[t]{2}{*}{ WHOI/XRF } & 61.6 & 63.0 & 63.7 & 62.6 & Neg. & .8 & $.69 \mathrm{NS}$ \\
\hline & 62.7 & 62.0 & 62.5 & & & & \\
\hline \multirow[t]{2}{*}{ UWUrz/XRF } & 63 & 67 & 60 & 64 & 2.9 & 1.2 & 13.50 \\
\hline & 65 & 67 & 62 & & & & \\
\hline \multirow[t]{3}{*}{ Liege/XRF } & 67 & 69 & - & 68.8 & Neg. & 2.0 & .39NS \\
\hline & 69 & 69 & - & & & & \\
\hline & 72 & 67 & - & & & & \\
\hline
\end{tabular}




\begin{tabular}{|c|c|c|c|c|c|c|c|}
\hline \multirow[b]{3}{*}{ Oxge/Methe } & \multirow{2}{*}{\multicolumn{2}{|c|}{ Botele }} & \multirow[b]{2}{*}{ Number } & \multirow[b]{3}{*}{ Mean } & \multicolumn{2}{|c|}{ Standard Deviation } & \multirow{3}{*}{$\begin{array}{r}\mathbf{P} \\
\text { ratio } \\
\end{array}$} \\
\hline & & & & & \multirow{2}{*}{$\begin{array}{l}\text { Bottle } \\
2 \mathrm{df} \\
\end{array}$} & \multirow{2}{*}{$\frac{\text { Brror }}{3 \mathrm{df}}$} & \\
\hline & 1 & 2 & 3 & & & & \\
\hline & & & & ont.) & & & \\
\hline $\mathrm{B} 1 \mathrm{TW} / \mathrm{XRP}$ & 62.1 & 62.2 & - & 62.6 & 0.77 & 1.32 & $2.01 \mathrm{NS}$ \\
\hline & 63.2 & 62.9 & - & & & & \\
\hline & 64.7 & 60.3 & - & & & & \\
\hline Nott/XRF & 71.27 & 70.38 & - & 69.13 & 1.23 & 2.87 & $1.55 \mathrm{NS}$ \\
\hline & 65.03 & 68.24 & - & & & & \\
\hline & 66.71 & 73.16 & - & & & & \\
\hline Curfe/XRF & 57.03 & 58.49 & - & 57.46 & .69 & .75 & $3.59 \mathrm{NS}$ \\
\hline & 57.40 & $57 \cdot 04$ & - & & & & \\
\hline & 56.22 & 58.59 & - & & & & \\
\hline UInd/ICPS & 71 & 72 & 72 & 72.5 & Neg. & 1.7 & $.18 \mathrm{NS}$ \\
\hline & 75 & 72 & 73 & & & & \\
\hline Exxon/DCPAS & 137.4 & 72.2 & - & 88.5 & 4.1 & 24.1 & $1.08 \mathrm{NS}$ \\
\hline & $77 \cdot 1$ & 84.3 & - & & & & \\
\hline & 81.8 & 78.3 & - & & & & \\
\hline USGSR/OES & 72 & 79 & 78 & 79 & Neg. & 5.5 & $.46 \mathrm{NS}$ \\
\hline & 84 & 85 & 76 & & & & \\
\hline *HMI/SSMS & 87.6 & 87.6 & - & 87.8 & 4.4 & 6.3 & $2.00 \mathrm{NS}$ \\
\hline & 96.9 & 79.1 & - & & & & \\
\hline & $109 \cdot 2$ & - & - & & & & \\
\hline GCL/AAS & 75 & 75 & 75 & 72.3 & Neg. & 4.0 & . $\mathrm{L}_{4} \mathrm{NS}$ \\
\hline & 69 & 72 & 68 & & & & \\
\hline ETH/AAS & 71.6 & 71.5 & - & 71.6 & - & - & - \\
\hline$/ \mathrm{XRF}$ & 67 & 66 & 69 & 67 & - & - & - \\
\hline KJeIl/Aas & 67 & 66 & 68 & 67 & - & - & - \\
\hline & & & $\mathbf{z r}$ & & & & \\
\hline WHOI/XRP & 35.3 & 35.7 & 35.6 & 35.3 & Neg. & .5 & $.53 \mathrm{NS}$ \\
\hline & 34.9 & 34.6 & 35.5 & & & & \\
\hline UWHIrz/XRF & 34 & 32 & 32 & 32.8 & 1 & .4 & 13.00 \\
\hline & 34 & 33 & 32 & & & & \\
\hline $\mathrm{WSU} / \mathrm{XRF}$ & 8 & 2 & 0 & $\sim 2$ & - & - & - \\
\hline & 0 & 2 & 3 & & & & \\
\hline$B \perp r m / X R F$ & 16.5 & 16.6 & - & 16.8 & Neg. & .31 & $.28 \mathrm{NS}$ \\
\hline & 16.8 & $17 \cdot 3$ & - & & & & \\
\hline & 16.8 & 16.6 & - & & & & \\
\hline Nott/XRP & 20.60 & 20.50 & - & 21.66 & Neg. & 1.89 & $.02 \mathrm{NS}$ \\
\hline & 21.71 & 19.86 & - & & & & \\
\hline & 23.04 & 24.27 & - & & & & \\
\hline Tohok/IPAA & 16 & 18 & 16 & 16.7 & .64 & .58 & $3.50 \mathrm{NS}$ \\
\hline & 17 & 17 & 16 & & & & \\
\hline USGSR/OES & 24 & 25 & 24 & 25.8 & Neg. & 2.4 & $.11 N S$ \\
\hline & 27 & 26 & 29 & & & & \\
\hline $\mathrm{ETH} / \mathrm{XRF}$ & 18 & 17 & 18 & 18 & - & - & - \\
\hline
\end{tabular}

Notes for tables 1-3

Critical values of the $F$ ratio for several probabilities and degrees of freedom

\begin{tabular}{|c|c|c|c|}
\hline \multirow[b]{2}{*}{$\begin{array}{c}\text { Degrees of } \\
\text { freedom }\end{array}$} & \multicolumn{3}{|c|}{ Probability } \\
\hline & 0.05 & 0.025 & 0.01 \\
\hline$(1,2)-$ & 18.5 & 38.5 & 98.5 \\
\hline - & 10.1 & 12.2 & 21.2 \\
\hline .......... & 9.55 & 16.0 & 30.8 \\
\hline$(2,9)$ & 4.26 & 5.71 & 8.02 \\
\hline
\end{tabular}

BIO:

Marine Ecology Laboratory, Bedford Institute of Oceanography, Dartmouth, N.S., Canada B2Y 4A2.

Analyst: R. T. T. Rantala.
Method: Sample portions were decomposed by acid in Teflon bombs (Rantala and Loring, 1973), and the constituents were determined by flame emission absorption spectroscopy (Rantala and Loring, 1975).

Birm: Department of Geological Sciences, University of Birmingham, P.O. Box 363, Birmingham, B15 2TT, England.

Analyst: G. L. Hendry.

Method: Ten grams of rock powder were mixed with 20 drops of a 7 percent aqueous solution of polyvinyl alcohol, and the mixture was compressed at 15 tons between polished faces of a 46-mm diameter steel die. Pellets were dried overnight at $110^{\circ} \mathrm{C}$ before analysis.

The elements were determined by the method of Leake and others (Leake and others, 1969), using a Philips PW 1450' automatic wavelength dispersive X-ray fluorescence spectrometer with a $\mathbf{L i F}_{220}$ crystal and a proportional or a scintillation counter. The gas for the proportional counter was $90 \%$ argon $10 \%$ methane.

(a) For $\mathrm{Ni}, \mathrm{Cr}, \mathrm{Zr}$, and $\mathrm{Nb}$, a tube with an $\mathrm{Rh}$ anode was operated at $70 \mathrm{kV}$ and $30 \mathrm{~mA}$ with a $0.15-\mathrm{mm}$ collimater. A proportional counter was used for $\mathrm{Ni}$ and $\mathrm{Cr}$ and a scintillation counter for $\mathrm{Zr}$ and $\mathrm{Nb}$. Standards were prepared from pure oxide powders, or solutions of elements were added to rock and silica powders.

(b) A tube with an Mo anode, used for $\mathrm{Y}, \mathrm{Sr}$, $\mathrm{Rb}, \mathrm{Th}, \mathrm{Pb}, \mathrm{Ga}, \mathrm{Zn}$, and $\mathrm{Ba}$, was operated at $60 \mathrm{kV}$ and $30 \mathrm{~mA}$ with a $0.55-\mathrm{mm}$ collimator for Ba but a $0.15-\mathrm{mm}$ collimator for the other elements. The proportional counter was used for $\mathrm{Ga}, \mathrm{Zn}$, and $\mathrm{Ba}$ and the scintillation counter for the other elements. In addition to standards as in (a), samples whose $\mathrm{Rb}, \mathrm{Sr}$, and $\mathrm{Ba}$ contents had been determined by IDMS were used.

(c) $\mathrm{Ce}, \mathrm{La}$, and $\mathrm{Nd}$ were determined with an $\mathrm{X}$-ray tube with a W anode operated at $60 \mathrm{kV}$ and $30 \mathrm{~mA}$, with a $0.55-\mathrm{mm}$ collimator and the flow counter. Standards were samples analyzed for their $\mathrm{Ce}, \mathrm{La}$, and Nd contents by IDMS.

BMNH: Department of Mineralogy, British Museum of Natural History, Cromwell Road, London, SW7 5BD, England.

Analysts: G. Jones, C. Elliott, V. Din.

Methods: (data were obtained on sample portions dried at $110^{\circ} \mathrm{C}$ for 2 hours).

Gravimetric: $\mathrm{SiO}_{2}, \mathrm{CaO}$ (corrected for $\mathrm{SrO}$ ), and $\mathrm{MgO}$ (corrected for $\mathrm{MnO}$ ).

Colorimetric: $\mathrm{TiO}_{2}$ was determined with Tiron, $\mathrm{Fe}_{2} \mathrm{O}_{3} \mathrm{~T}$ with sulfosalicylic acid, $\mathrm{MnO}$ with permanganate, and $\mathrm{P}_{2} \mathrm{O}_{5}$ with molybdenum blue

\footnotetext{
${ }^{1}$ Trade names are used for identification only and do not constitute endorsement by the U.S. Geological Survey.
} 
in either (a) a solution of a $\mathrm{KHSO}_{4}$ fusion of the ignited $\mathrm{R}_{2} \mathrm{O}_{3}$ precipitate or (b) an $\mathrm{H}_{2} \mathrm{SO}_{4}$ solution of the rock sample after removal of $\mathrm{SiF}_{4}$ by $\mathrm{HF}$ and $\mathrm{H}_{2} \mathrm{SO}_{4} . \mathrm{Al}_{2} \mathrm{O}_{3}$ was found by difference after either type of solution.

Titrimetric: The sample was dissolved in $\mathrm{HF}-\mathrm{H}_{2} \mathrm{SO}_{4}$ in a sealed polycarbonate bottle and, after adding $\mathrm{H}_{3} \mathrm{BO}_{3}$, ferrous iron was titrated with standardized $\mathrm{KMnO}_{4}$.

$\mathrm{C}, \mathrm{H}, \mathrm{N}$ elemental analyzer: Total $\mathrm{C}$ and total $\mathrm{H}$ were calculated to $\mathrm{CO}_{2}$ and $\mathrm{H}_{2} \mathrm{O}$, respectively.

Ion-selective electrode: After pyrohydrolytic separation from a rock sample mixed with a flux, $F$ was determined.

Atomic absorption: Constituents were determined in $\mathrm{HF}-\mathrm{H}_{3} \mathrm{BO}_{3}$ solutions containing $200 \mathrm{mg}$ of rock sample in $100 \mathrm{~mL}$. Synthetic or reference samples prepared similarly were used for calibration.

X-ray fluorescence: Constituents were determined in glass discs prepared in pairs from one fusion mixture of $1000 \mathrm{mg}$ of rock sample with $4000 \mathrm{mg}$ of lithium metaborate. All discs were analyzed against reference samples prepared similarly.

* BMNH/Chem: Three analysts each made two of the six determinations. The average was used for best values but the data were not used for the analysis of variance.

* BMNH/XRF: The separate sets of data were made by different analysts.

Chels: $\quad$ Department of Geology, Chelsea College, 271 King Street, London, W6 9LZ, England (Gill); Department of Geology, Imperial College, Prince Consort Road, London, SW7, England (Rogers).

Analysts: R. C. O. Gill and N. W. Rogers.

Method: 200-mg portions of W-2 and DNC-1, and $100-\mathrm{mg}$ portions of BCR-1, were weighed into capsules. Other capsules contained $100 \mu \mathrm{L}$ of standard solutions evaporated onto a $1-\mathrm{cm}$ filter paper (Borley and Rogers, 1979). Standard solutions contained rare-earth elements in the same proportions as those in BCR-1. The set of capsules was divided into two groups of 12 for irradiation, preserving random order. Capsules with standard solutions were placed at the bottom, middle, and top of each group of 12 capsules to correct for neutron flux gradients.

Samples were irradiated simultaneously in the "Consort Mk II" reactor at the University of London Reactor Centre, Silwood Park, Ascot, Berkshire, for $40 \mathrm{hr}$ at a thermal flux of $10^{12} \mathrm{n} \mathrm{cm}^{-2} \mathrm{~s}^{-1}$. A Princeton Gamma-Tech (Li) detector of $42 \mathrm{~cm}^{3}$ active volume, with a resolution of $1.81 \mathrm{keV}$ FWHM (full width-half maximum) at $1.33 \mathrm{meV}$, was used for energies $>200 \mathrm{keV}$, and a Princeton Gamma-Tech ultrapure Ge detector with an active area of $200 \mathrm{~mm}^{2}$ and $5 \mathrm{~mm}$ thick, with a resolution of $520 \mathrm{eV}$ at $122 \mathrm{keV}$, was used for energies $<210 \mathrm{keV}$.

$\mathrm{La}, \mathrm{Lu}, \mathrm{Ho}$, and Sm were determined about 3 days after irradiation when short-lived isotopes had decayed, and the remaining elements were determined after another delay of 3 weeks when other interfering isotopes had decayed.

CRPG: Centre de Recherches Pétrographiques et Géochimiques, C. O. No. 1, 54500 Vandoeuvre-les-Nancy, France.

Analyst and method: K. Govindaraju and others (1976).

Curie: Laboratoire de Géologie Appliquée, Université Pierre et Marie Curie, 4, Place Jussieu, 75230 Paris, CEDEX 05, France.

Analyst: M. Quintin.

Method: Quintin and others (1978). Synthetic standards were made from $\mathrm{CuO}, \mathrm{Ga}_{2} \mathrm{O}_{3}$, and ZnO (Johnson Matthey Chemicals Limited).

ETH: $\quad$ Eidg. Technische Hochschule, ETH-Zentrum, CH-8092, Zürich, Switzerland.

Analysts: B. Ayranci, major and minor oxides, plus $\mathrm{Cr}, \mathrm{Cu}, \mathrm{Ni}, \mathrm{Sr}$, and $\mathrm{Zn}$ by atomic absorption and $\mathrm{H}_{2} \mathrm{O}$ by Penfield; V. Dietrich, major and minor oxides plus trace elements by $\mathrm{X}$-ray fluorescence, and $\mathrm{Ba}$ by IDMS.

Exxon: Exxon Production Research Co., P.O. Box 2189, Houston, Texas 77001.

Analyst: P. E. Drez.

Method: Spectrametric's Spectrascan IIIA DC arc plasma emission spectrometer was used in the multielement mode with a 3-electrode spectrajet. 50-mg portions of rock in sealed polypropylene centrifuge tubes were dissolved with $3 \mathrm{~mL} \mathrm{HF}$ and $2.8 \mathrm{~g} \mathrm{H}_{3} \mathrm{BO}_{3}$. Solutions were diluted so that the final concentration was $500 \mathrm{ppm}$ of whole rock. $2000 \mathrm{ppm}$ of Cs was added as an ionization buffer. Nineteen reference rocks were treated the same as the unknowns and were used as standards.

GCL: Government Chemical Laboratories, 30 Plain Street, Perth, Western Australia 6000.

Analysts: C. J. Dood (Cd and Zn); R. S. Y. Pepper (Be); and E. J. Tovey (As and B).

Methods: Cd and $\mathrm{Zn}-\mathrm{AAS}$. Sample portions were dissolved with $\mathrm{HF}, \mathrm{HNO}_{3}$, and $\mathrm{HClO}_{4}$ and taken to fumes. The residue was diluted to volume and $\mathrm{Zn}$ determined by atomic absorption. Cd was complexed with diethyl dithiocarbamate and extracted into chloroform. After evaporating the chloroform, the residue was dissolved in $\mathrm{HClO}_{4}$ and diluted to volume before determining $\mathrm{Cd}$ by atomic absorption. An air- $\mathrm{C}_{2} \mathrm{H}_{2}$ flame was used for both elements. $\mathrm{Be}-\mathrm{AAS}$. Samples were fused with $\mathrm{KHF}_{2}$ and the fusion treated with $\mathrm{H}_{2} \mathrm{SO}_{4}$ and brought to fumes. The residue was taken up with $\mathrm{HCl}$ and 
diluted to volume to determine $\mathrm{Be}$ by atomic absorption with an $\mathrm{N}_{2} \mathrm{O}-\mathrm{C}_{2} \mathrm{H}_{2}$ flame.

B-Spectrophotometry. Samples were decomposed by sintering with $\mathrm{Na}_{2} \mathrm{CO}_{3}-\mathrm{ZnO}$ and an aqueous extract was acidified with $\mathrm{HCl}$. After complexing fluorine with zirconyl chloride, the boron was extracted into a chloroform solution of 2-ethylhexane-1,3 diol (EHD). The chloroform was evaporated from the organic extract, leaving a residue containing the boron. After adding curcumin in acetic acid solution to the EHD residue, $\mathrm{H}_{2} \mathrm{SO}_{4}$ was also added, resulting in a boron complex (rosocyanin) in about 75 $\mathrm{min}$. This solution was diluted to volume with ethanol and boron determined spectrophotometrically at $555 \mathrm{~nm}$.

As-Spectrophotometry. Sample portions were fused with $\mathrm{Na}_{2} \mathrm{CO}_{3}-\mathrm{Na}_{2} \mathrm{O}_{2}$ and a water extract taken. After adding $\mathrm{HCl}$ and $\mathrm{KI}$, the As was reduced with $\mathrm{SnCl}_{2}$ and further reduced to arsine by hydrogen generated by $\mathrm{HCl}$ on $\mathrm{Zn}$ metal. After removing any $\mathrm{H}_{2} \mathrm{~S}$, the arsine was absorbed into a solution in which it was oxidized to arsenate by iodine. The arsenate was converted to arsenomolybdate, which was reduced by hydrazine sulfate to a molybdenum blue whose absorbance was measured.

Genev: Department of Mineralogy, University of Geneva, 13, rue des Maraíchers, 1211 Geneva 4, Switzerland.

Analyst: P. Voldet.

Method: Voldet and Haerdi (1978).

GSC: Geological Survey of Canada, 601 Booth Street, Ottawa, Canada K1A 0E8. An asterisk $\left({ }^{*}\right)$ following a letter for a method in the tables indicates the method preferred by GSC. Methods:

A: Sample portions were fused with lithium tetraborate-fluoride mixture and were cast into discs. The discs were irradiated in a programmable wavelength-dispersive X-ray fluorescence spectrometer. Peak and background intensities were processed by an off-line computer, referred to stored calibrations, and corrected for interelement effect and instrumental drift.

B: Modified Wilson vanadate method (Maxwell, 1968 , p. 419-421).

C: By difference between total $\mathrm{Fe}(\operatorname{method} \mathrm{A})$ and $\mathrm{Fe}(\mathrm{II})$ (method B).

D: Sample was fused with lithium metaborate and the fusion was disintegrated with dilute $\mathrm{HCl}$. The solution was evaporated with $\mathrm{HCl}$ and methanol to dehydrate silica and volatilize boron. Silica was determined gravimetrically as $\mathrm{SiO}_{2}$. The residue from the HF treatment was fused with pyrosulfate and set aside for the $\mathrm{Ti}$ determination. Unprecipitated silica was determined colorimetrically in the filtrate with molybdenum blue.

E: Determined in the filtrate from $D$ by atomic absorption spectroscopy with an $\mathrm{N}_{2} \mathrm{O}-\mathrm{C}_{2} \mathrm{H}_{2}$ flame.

F: Pratt method with potentiometric titration (Maxwell, 1968, p. 419, 423).

G: Same method as E, with an air- $\mathrm{C}_{2} \mathrm{H}_{2}$ flame.

$\mathrm{H}$ : The pyrosulfate fusion from $\mathrm{D}$ was dissolved in dilute sulfuric acid. The corresponding aliquots from that solution and the main filtrate from $D$ were combined, $\mathrm{AlCl}_{3}$ and $\mathrm{HCl}$ were added, and $\mathrm{Ti}$ was determined as in $\mathrm{E}$.

$\mathrm{J}$ : Determined in the filtrate from $\mathrm{D}$ with molybdenum blue.

K: Sample was fused with $\mathrm{Na}_{2} \mathrm{CO}_{3}$ and the fused melt leached with water. $\mathrm{F}$ was determined by an ion-selective electrode in the presence of a buffer.

L: Colorimetric determination with mercuric thiocyanate and ferric iron on an aliquot from $\mathrm{K}$.

M: Determined by atomic absorption spectroscopy on a solid sample by a "screw-rod" method (Bouvier and Abbey, 1977).

$\mathrm{N}$ : Sample was mixed with buffer and graphite and excited in an air-jet controlled dc arc. The emitted radiation was dispersed in a directreading optical spectrometer. The signals generated in individual photomultiplier tubes were processed automatically by an on-line mini-computer.

U: Uranium was separated by solvent extraction and determined fluorimetrically.

$\mathrm{X}: \mathrm{Rb}$ and $\mathrm{Sr}$ were determined on unfused powders by comparison with similar standards in a manual X-ray fluorescence spectrometer.

$\mathrm{Y}$ : The sample was mixed with $\mathrm{V}_{2} \mathrm{O}_{5}$ and then heated in a stream of oxygen. Evolved gases were analyzed by the integration of the signals generated in non-dispersive infrared detectors.

Z: Sample was mixed with lead oxide and heated in a Penfield tube (Maxwell, 1968, p. 426). The evolved water was dissolved in an organic solvent and titrated with Karl Fisher reagent.

a: Gases evolved by treating a sample with $\mathrm{HCl}$ were passed through traps to remove interferences. $\mathrm{CO}_{2}$ was absorbed in an organic solvent and determined by non-aqueous acidimetric titration in a modified sulfur titrator (Bouvier and others, 1972).

b: Conventional drying in an oven.

c: As and $\mathrm{Sb}$ were determined as hydrides by atomic absorption spectroscopy after leaching the sample with aqua regia and diluting an aliquot with $0.5 \mathrm{M} \mathrm{HCl}$. 
Analysts and methods used

\begin{tabular}{|c|c|}
\hline Analyst & Method \\
\hline \multirow{2}{*}{\multicolumn{2}{|c|}{$\begin{array}{l}\text { J. L. Bouvier - } \\
\text { V. E. Grushman - }-1, F, Y, b \\
\end{array}$}} \\
\hline & \\
\hline \multicolumn{2}{|c|}{ A. G. Douma - } \\
\hline \multicolumn{2}{|c|}{ R. M. Rousseau - } \\
\hline \multicolumn{2}{|c|}{ N. Bertrand - } \\
\hline \multicolumn{2}{|c|}{ F. J. Watson - } \\
\hline \multicolumn{2}{|c|}{ P. G. Belanger - } \\
\hline \multicolumn{2}{|c|}{ Gillis Gauthier -_-_-_ c } \\
\hline \multicolumn{2}{|c|}{ Serge Courville -______-_ U } \\
\hline \multicolumn{2}{|c|}{ R. J. Guillas - -} \\
\hline \multicolumn{2}{|c|}{$\begin{array}{l}\text { K. A. Church - } \\
\text { R. A. Meeds - }\end{array}$} \\
\hline R. A. Meeds - - - & $\mathbf{N}$ \\
\hline
\end{tabular}

GSF:

HMI:

Geological Survey of Finland, SF-02150 Espoo 15, Finland.

Analysts: Chemical analysis, Risto Saikonen (W-2), Mervi Wiik (DNC-1), Christer Ahlsved (BIR-1); optical emission spectroscopy, A. spectroscopy, O. Kuovo.

Methods for chemical analysis (pages refer to Maxwell, 1968): Gravimetric: $\mathrm{SiO}_{2}$ (p. 323-332, 348-350); $\mathrm{CaO}$ (p. 363-367); $\mathrm{MgO}$ (p. 372374); $\mathrm{H}_{2} \mathrm{O}^{+}$(p. 426-430).

Colorimetric: $\mathrm{TiO}_{2}$ (p. 379-383); $\mathrm{MnO}$ (p. 387389); $\mathrm{P}_{2} \mathrm{O}_{5}$ (p. 392-394); $\mathrm{Fe}_{2} \mathrm{O}_{3}$ as the yellow 1.14- $\mathrm{HCl}$ complex.

Titrimetric: $\mathrm{FeO}$ (p. 416-418).

Atomic absorption: $\mathrm{Al}_{2} \mathrm{O}_{3}, \mathrm{MgO}, \mathrm{CaO}, \mathrm{K}_{2} \mathrm{O}$. Flame emission: $\mathrm{Na}_{2} \mathrm{O}$.

Hahn-Meitner Institut, Postfach 3901 28,
Kjell:

LASL:

Liège: Puisto and R. Danielson; isotope dilution mass D-1000 Berlin 39, Federal Republic of Germany.

Analysts: INAA-F. Schley, Bereich Kernchemie und Reaktor.

SSMS-J. Luck and W. Szacki, Department of Geochemistry.

Methods: INAA: Samples were dried at $100^{\circ} \mathrm{C}$ and 100-150 mg portions were sealed in highpurity quartz ampoules. Samples were irradiated for $48 \mathrm{hr}$ in a flux of $\sim 5 \times 10^{12} \mathrm{n} \mathrm{cm}^{-2}$ $\mathrm{s}^{-1}$. Elements were measured, against either W-1 (INAA-W) or BCR-1 (INAA-B) as standards, by gamma-ray spectroscopy for 2000 $s$ after a decay of 4-5 days and for $7000 \mathrm{~s}$ after a decay of 30 days. Resolution of the detector was $1.9 \mathrm{keV}$ at $1332 \mathrm{keV}$. SSMS: The operating conditions for the mass spectrometer were: spark voltage, $40 \mathrm{kV}$; high tension, $20 \mathrm{kV}$; magnet current, $300 \mathrm{~mA}$; source pressure, $10^{-6}-10^{-7} \mathrm{Torr}$; and analysis pressure, $2-4 \times$ $10^{-8}$ Torr. The determinations were made on a MS 702 with photoplate detection on Ilford Q-2 plates with 16 exposures per plate from
$0.02 \mathrm{nC}$ to $100 \mathrm{nC}$. Photoplates were developed by a modified ID 13 developer and measured with an Optronics $S 3000$ densitometer with an $\alpha-16$ microcomputer and a Kennedy tape machine.

Institute for Energy Technology, 2007 Kjeller, Norway.

Analysts: A. Follo and E. Steinnes.

University of California, Los Alamos National Laboratory, P.O. Box 1663, Los Alamos, New Mexico 87545.

INAA-1 and DNAA-1, analyst, Ernest $S$. Gladney.

INAA and XRF, analysts, T. J. Bornhorst and J. P. Balanga.

Géologie, Pétrologie, et Géochimie, Université de Liège, B-4000 Sart-Tillman par Liège 1, Belgium.

Analysts: G. Bologne and I. Roelandts.

Methods: $\mathrm{FeO}$ was determined volumetrically and $\mathrm{H}_{2} \mathrm{O}^{+}$and $\mathrm{H}_{2} \mathrm{O}^{-}$gravimetrically on three portions of sample from each bottle. X-ray fluorescence: three measurements were made for each trace element on one pressed powder pellet from each bottle. The error term for $\mathrm{FeO}, \mathrm{H}_{2} \mathrm{O}^{+}$, and $\mathrm{H}_{2} \mathrm{O}^{-}$is the error within separate portions of sample whereas the error term for the trace elements is the error within the triplicate measurements of each pressed powder pellet.

Munich: Mineralogisch-Petrologisches Institut, Universität München, Theresienstrasse 41, 8 Munich 2, Federal Republic of Germany.

Analysts: G. Troll and A. Farzaneh. Reference: Farzaneh and Troll $(1977,1978)$.

NERF: $\quad$ Research Centre, Netherlands Energy Research Foundation, 3, Westerduinweg, Petten (NH), The Netherlands.

Analysts: H. A. van der Sloot and H. A. Das. Method: Si was determined by $14-\mathrm{meV}$ neutron activation with a Sames neutron generator. An irradiation for $10 \min$ at $10^{11} \mathrm{n} \mathrm{cm}^{-2} \mathrm{~s}^{-1}$ was used for $\mathrm{Na}, \mathrm{K}, \mathrm{Mn}$, and $\mathrm{Dy}$, and an irradiation for $1 \mathrm{~min}$ at the same flux for Al. For other elements, samples of about $1 \mathrm{~g}$ were irradiated for $2 \mathrm{hr}$ at a flux of $3 \times 10^{12} \mathrm{n} \mathrm{cm}^{-2}$ $\mathrm{s}^{-1}$.

NIM: $\quad$ National Institute for Metallurgy, Private Bag X3015, Randburg, 2125, South Africa.

Methods: X-ray fluorescence spectroscopy. A subsample of $1 \mathrm{~g}$ was fused with a mixture of lithium tetraborate, sodium tetraborate, and sodium carbonate. A glass disc was cast and the sample evaluated using influence factors. (There are 9 d.f. for the error standard deviation for these data.)

Atomic emission spectroscopy: A subsample of 
$0.2 \mathrm{~g}$ was fused with a mixture of $\mathrm{LiBO}_{2}$, $\mathrm{LiNO}_{3}$, and $\mathrm{B}_{2} \mathrm{O}_{3}$. After the fusion was put into solution, the solution was analyzed with a Hilger spectrometer using inductively coupled plasma excitation.

Atomic absorption spectroscopy: A subsample of $1 \mathrm{~g}$ was attacked with $\mathrm{H}_{2} \mathrm{SO}_{4}, \mathrm{HClO}_{4}$, and $\mathrm{HF}$ in a platinum dish and the residue was dissolved in $\mathrm{HCl}$ and $\mathrm{HNO}_{3}$. The elements were measured on a Varian-Techtron AAS using a $\mathrm{N}_{2} \mathrm{O}-\mathrm{C}_{2} \mathrm{H}_{2}$ flame.

Neutron activation analysis: Subsamples of $0.5 \mathrm{~g}$ were irradiated for 3 hours and counted at decay times ranging from 3 to 30 days. The data were processed with a modified version of the Yule program. Flux variations were corrected by normalizing counts against monitor wires around standard vials.

Ferrous oxide: Subsamples of $0.15 \mathrm{~g}$ were fused with sodium metafluoborate and sodium vanadate in an atmosphere of $\mathbf{N}_{2}$. Fusions were leached in water and $\mathrm{H}_{2} \mathrm{SO}_{4}$ and the solutions were titrated with ferrous ammonium sulfate.

Total iron: Subsamples of $0.5 \mathrm{~g}$ were fused in sodium peroxide, the fusions were leached with water, and the resulting solutions were acidified with $\mathrm{HCl}$ after an $\mathrm{R}_{2} \mathrm{O}_{3}$ precipitation and dissolution of the hydroxides. The iron was determined by titration with potassium dichromate.

Nott: Department of Geology, University of Nottingham, University Park, Nottingham, NG7 2RD, England.

Analyst: P. K. Harvey.

Method: The determinations were made on pressed powder pellets, and a modified Compton scatter ratio technique was used.

Open: Department of Earth Sciences, The Open University, Walton Hall, Milton Keynes, MK7 6AA, England.

Analysts: P. J. Potts and O. W. Thorpe.

Method: Instrumental neutron-activation analysis.

Parma: Istituto di Mineralogia, Universita degli Studi di Parma, Via A. Gramsci 9, 43100 Parma, Italy.

Analysts: G. Di Battistine, F. Gallo, G. Venturelli, L. Vernia, L. Beccaluva, and F. Emiliani.

Methods:

Gravimetric: $\mathrm{SiO}_{2}$.

Colorimetric: $\mathrm{TiO}_{2}$ and $\mathrm{Fe}_{2} \mathrm{O}_{3}$ (Casanova and others, 1968); $\mathrm{P}_{2} \mathrm{O}_{5}$ (Shapiro and Brannock, 1962).

Flame photometric: $\mathrm{Na}, \mathrm{K}$.

Atomic absorption spectroscopy: $\mathrm{Al}, \mathrm{Co}, \mathrm{Cr}$, $\mathrm{Cu}, \mathrm{Li}, \mathrm{Ni}, \mathrm{Sr}$, and $\mathrm{V}$. Two analysts determined V.
TexA\&M: Department of Geology, Texas A\&M University, College Station, Texas 77843.

E. B. Ledger, T. T. Tieh, and M. W. Rowe determined uranium in $\mathrm{W}-2$ by delayed neutron activation analysis.

The uranium contents of DNC-1 and BIR-1 were below the limit of estimation (Ledger and others, 1980).

Tohok: Department of Chemistry, Tohoku University, Sendai, Japan 980. Instrumental photon activation analysis by $\mathbf{T}$. Kato and $\mathbf{H}$. Yokobayashi.

Method: Kato and others (1977); Masumoto and others (1978).

Toron: SLOWPOKE Reactor Office, University of Toronto, Toronto, Canada M5S 1A4.

Analyst: R. G. V. Hancock.

Method: Hancock (1976). The third line of data was discarded for the analysis of variance of Ba data in W-2, and there are 3 d.f. for the error s.d. The first set of Eu data was obtained using ${ }^{152 \mathrm{~m}} \mathrm{Eu}$.

UInd: Department of Geology, University of Indiana, Bloomington, Indiana 47405.

Analyst: P. L. Lechler.

USCSM: $\quad$ U.S. Geological Survey, 345 Middlefield Road, Menlo Park, California 94025.

Flame photometric determinations by $\mathbf{P}$. R. Klock and B. Lai.

USCSD: U.S. Geological Survey, Federal Center, Denver, Colorado 80225.

Determinations of $\mathrm{Na}_{2} \mathrm{O}$ and $\mathrm{Li}$ by atomic $\mathrm{ab}-$ sorption spectroscopy by V. M. Merritt.

Determination of $\mathrm{U}$ and $\mathrm{Th}$ by delayed neutron activation analysis by $H$. T. Millard, Jr. (Millard, 1976).

USGSR: U.S. Geological Survey, Reston, Virginia 22092.

Instrumental neutron activation analysis (Baedecker and others, 1977) by L. J. Schwarz. Atomic absorption spectrometry: $\mathrm{Mg}, \mathrm{Na}, \mathrm{K}$, and $\mathrm{Cu}$ by $\mathrm{W}$. M. d'Angelo; Li by $\mathrm{A}$. K. Neuville.

Flameless atomic absorption spectrometry: Ag and Ti by W. M. d'Angelo.

Spectrophotometry: Mo and $\mathrm{W}$ by $\mathbf{W}$. $M$. d'Angelo; Nb by E. Y. Campbell.

Rapid rock analysis (Shapiro, 1975) by Z. A. Hamlin.

Optical emission spectroscopic determinations by $\mathrm{N}$. Rait using methods modified from Bastron and others (1960).

X-ray fluorescence determinations by $\mathbf{R}$. B. Johnson using methods modified from Rose and others (1963).

UWürz: Institut für Mineralogie und Kristallstrukturlehre, Universität Würzburg, Federal Republic of Germany. 
Analyst: P. Richter.

Methods:

Major and minor oxides (except $\mathrm{Na}_{2} \mathrm{O}$ ): $\mathrm{X}$-ray fluorescence measurements after $\mathrm{Li}_{2} \mathrm{~B}_{4} \mathrm{O}_{7}$ fusion at 20:1 dilution.

$\mathrm{Na}_{2} \mathrm{O}$ and $\mathrm{Li}$ : flame atomic absorption.

$\mathrm{Cr}(<100 \mathrm{ppm})$ : flameless atomic absorption.

$\mathrm{Cr}(>100 \mathrm{ppm})$ and the remaining trace elements: X-ray fluorescence measurements on powder discs using internal standards or the method of standard additions.

WAIT: Department of Physics, Western Australian Institute of Technology, Hayman Road, South Bentley, Western Australia.

Analysts: K. J. R. Rosman and J. R. de Laeter. Method: Rosman and de Laeter (1980).

WHOI: Woods Hole Oceanographic Institution, Woods Hole, Massachusetts 02543.

Analysts: Geoffrey Thompson and Brian Schroeder.

Method: Major and minor constituents were determined on glass discs following the method of Norrish and Hutton (1969). Trace elements were determined on pressed powder pellets.

WSU:
Department of Geology, Washington State University, Pullman, Washington 99164.

$\mathrm{X}$-ray fluorescence determinations by $\mathbf{P}$. $R$. Hooper using the method of Hooper and Atkins (1969).
Table 4. Determinations of $\mathrm{Ni}, \mathrm{Rb}$, and $\mathrm{Sr}$ by $\mathrm{X}$-ray fluorescence

spectroscopy using two $X$-ray systems by $M$. Quintin[In parts per million.]

\begin{tabular}{|c|c|c|c|c|}
\hline \multirow[b]{2}{*}{ Sample } & \multirow[b]{2}{*}{ Bottle } & \multicolumn{2}{|c|}{ X-ray System } & \multirow[b]{2}{*}{ Mean } \\
\hline & & 1 & 2 & \\
\hline & \multicolumn{4}{|c|}{ Nickel } \\
\hline$W-2$ & $\begin{array}{l}1 \\
2\end{array}$ & $\begin{array}{l}66.24 \\
66.85\end{array}$ & $\begin{array}{l}79.35 \\
73.31\end{array}$ & 71.4 \\
\hline DNC-1 & $\begin{array}{l}1 \\
2\end{array}$ & $\begin{array}{l}249.28 \\
254.49\end{array}$ & $\begin{array}{l}239.27 \\
258.89\end{array}$ & 250.5 \\
\hline \multirow[t]{2}{*}{ BIR-1 } & $\begin{array}{l}1 \\
2\end{array}$ & $\begin{array}{l}155.07 \\
152.88\end{array}$ & $\begin{array}{l}165.08 \\
164.48\end{array}$ & 159.3 \\
\hline & \multicolumn{4}{|c|}{ Rubidium } \\
\hline$w-2$ & $\begin{array}{l}1 \\
2\end{array}$ & $\begin{array}{l}19.3 \\
17.9\end{array}$ & $\begin{array}{l}20.2 \\
19.9\end{array}$ & 19.3 \\
\hline DNC-1 & $\begin{array}{l}1 \\
2\end{array}$ & $\begin{array}{l}4.22 \\
4.10\end{array}$ & $\begin{array}{l}5.27 \\
5.64\end{array}$ & 4.80 \\
\hline \multirow[t]{2}{*}{ BIR-1 } & $\begin{array}{l}1 \\
2\end{array}$ & $\begin{array}{l}.42 \\
.42\end{array}$ & $\begin{array}{l}.58 \\
-\end{array}$ & .47 \\
\hline & \multicolumn{4}{|c|}{ Strontium } \\
\hline$W-2$ & $\begin{array}{l}1 \\
2\end{array}$ & $\begin{array}{l}217.09 \\
214.92\end{array}$ & $\begin{array}{l}213.69 \\
213.67\end{array}$ & 214.8 \\
\hline DNC-1 & $\begin{array}{l}1 \\
2\end{array}$ & $\begin{array}{l}163.00 \\
163.52\end{array}$ & $\begin{array}{l}160.52 \\
158.58\end{array}$ & 161.4 \\
\hline BIR-1 & $\begin{array}{l}1 \\
2\end{array}$ & $\begin{array}{l}120.46 \\
116.26\end{array}$ & $\begin{array}{l}115.54 \\
115.78\end{array}$ & 117.0 \\
\hline
\end{tabular}

1/ Laboratoire de Géologie Appliquée, Université Pierre et Marie Curie, 4, Place Jussieu, 75230 Paris CEUEX 05, France

2/ X-ray systenis: (1) Philips PWW 1450; (2) Siemens SRS 1. An Au tube with the Siemens SRS 1 was used for $\mathrm{Ni}$ but a Mo tube was used el sewhere

Standards: A synthetic standard for $N i$ was made from Johnson Matthey NiO. A synthetic standard with $1000 \mathrm{ppm} \mathrm{Sr}$ was made from Johnson Matthey $\mathrm{SrCO}_{3}$ and $\mathrm{SiO}_{2}$ (impurities $<0.01 \mathrm{ppm}$ ). USGS-G-2 (168 ppm) was used for Rb

Method: Quintin and others (1978)

Table 5. Determinations of trace elements in USGS-BIR-1 by spark source mass spectrometry by K. P. Jochum and M. Seufert, Max Planck Institut fur Chemie

[In parts per million. Calibration by RSF, relative sensitivity factors, and by ID, isotope dilution method.]

\begin{tabular}{|c|c|c|c|c|c|}
\hline Element & RSF & ID & Element & RSF & ID \\
\hline $\mathrm{Cu}$ & - & 129 & Sm & 0.94 & 1.06 \\
\hline $\mathrm{Rb}$ & 0.28 & & Eu & .62 & .564 \\
\hline$S r$ & 122 & 11 & Gd & 1.41 & 1.85 \\
\hline $\bar{y}$ & 19 & - & $\mathrm{Tb}$ & .26 & - \\
\hline $\mathrm{Zr}$ & 18 & - & Dy & 2.19 & 2.23 \\
\hline $\mathrm{Nb}$ & .78 & - & Ho & .45 & - \\
\hline$S n$ & .63 & .746 & Er & 1.58 & - \\
\hline $\mathrm{Sb}$ & .65 & - & $\mathrm{Tm}$ & .195 & - \\
\hline CS & $<.001$ & - & Yb & 1.14 & 1.27 \\
\hline $\mathrm{Ba}$ & - & 7.90 & Lu & .23 & - \\
\hline La & .55 & - & $\mathrm{Hf}$ & .48 & .484 \\
\hline $\mathrm{Ce}$ & 1.96 & - & $\mathrm{Pb}$ & 2.2 & 2.65 \\
\hline $\mathrm{Pr}$ & .35 & - & Th & $<.02$ & - \\
\hline Nd & 2.27 & 2.00 & U & $<.01$ & - \\
\hline
\end{tabular}

1 Abteilung Geochemie, Max Planck Institut fur Chemie, Saarstrasse 23, D-6500 Mainz, W. Germany 
Table 6. Deterainations of constituents by instrumental neutron activation malysis by A.Y. Muralid

\begin{tabular}{|c|c|c|c|c|c|c|c|c|c|}
\hline \multirow{2}{*}{$\begin{array}{l}\text { Sappie } \\
\text { Portion }\end{array}$} & \multicolumn{4}{|c|}{ Bottle } & \multicolumn{5}{|c|}{ muser } \\
\hline & $\mathrm{r}$ & $n$ & B & c & $i$ & $m$ & 8 & $c$ & meen \\
\hline \multicolumn{10}{|c|}{$n-2$} \\
\hline \multicolumn{10}{|c|}{ In percent } \\
\hline Fe & 7.00 & 6.85 & 6.70 & - & 7.85 & 7.38 & 7.18 & - & $7.16 \mathrm{c}$ \\
\hline $\mathrm{Al}_{2} \mathrm{O}_{3}$ & - & - & . & $12.82^{\mathrm{d}}$ & - & 12.71 & - & - & 12.76 \\
\hline $\operatorname{mg} 0$ & - & 8.36 & 7.38 & $8.73^{4}$ & 6.97 & 6.87 & 7.19 & - & $7.58 \mathrm{C}$ \\
\hline $\mathrm{m}_{2} \mathrm{O}$ & 2.089 & 2.110 & 2.040 & $2.116 \mathrm{e}$ & 2.100 & 2.110 & 2.181 & . & $2.105 \mathrm{c}$ \\
\hline $\mathrm{THO}_{2}$ & - & 1.17 & - & .990 & .96 & .99 & 1.08 & - & 1.04 \\
\hline mo & .115 & .148 & .165 & .1198 & .158 & .120 & .124 & - & .1386 \\
\hline \multicolumn{10}{|c|}{ In parts per milliton } \\
\hline ce & - & 20 & 20 & - & 24.8 & - & 20 & - & 21.2 \\
\hline$c_{0}$ & 38 & $\infty$ & 38 & - & 40 & 37 & 39 & - & $39 \mathrm{c}$ \\
\hline cr & 78 & 68 & 68 & - & $x$ & 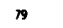 & $\pi$ & - & $x \mathrm{cc}$ \\
\hline Eu & - & .96 & .84 & - & 1.05 & .92 & .86 & - & .93 \\
\hline Hf & - & 1.4 & 2.5 & - & 1.8 & - & 2 & - & 1.9 \\
\hline LA & 10.15 & 10.30 & 10.50 & - & 9.40 & 11.15 & 10.40 & - & $10.32^{\mathrm{C}}$ \\
\hline Lu & .35 & .34 & .39 & - & .32 & .36 & .30 & - & $.34 \mathrm{C}$ \\
\hline$n_{1}$ & - & 9 & 11 & - & - & - & - & . & 10 \\
\hline sc & 30 & 31 & 30 & - & 33 & 29 & 33 & - & $31^{c}$ \\
\hline sm & 3.30 & 4.00 & - & - & 2.70 & 2.99 & 2.65 & - & 3.13 \\
\hline to & - & - & .5 & - & .57 & - & .61 & - & .56 \\
\hline Th & - & 1.25 & - & - & 1.4 & - & 1.2 & - & 1.28 \\
\hline$v$ & - & 266 & 259 & $252^{\circ}$ & 242 & 242 & 267 & - & $255 \mathrm{C}$ \\
\hline ro & 2.22 & 2.00 & 1.99 & - & 2.4 & 2.26 & 2.4 & - & $2.21 \mathrm{c}$ \\
\hline \multicolumn{10}{|c|}{ ONC-1 } \\
\hline \multicolumn{10}{|c|}{ In percent } \\
\hline fe & 6.62 & - & 6.78 & - & - & 6.82 & 6.89 & - & 6.78 \\
\hline $\mathrm{Al}_{2} \mathbf{O}_{3}$ & - & 15.05 & - & 15.99 & 15.36 & - & - & - & 15.46 \\
\hline NoO & 10.80 & 11.85 & 10.13 & 12.596 & 12.54 & 11.48 & 12.79 & - & $11.60 \mathrm{c}$ \\
\hline $\mathrm{Na}_{2} \mathrm{O}$ & 1.830 & 1.819 & 1.640 & $1.876^{\circ}$ & 1.829 & 1.890 & 1.910 & - & $1.820 \mathrm{c}$ \\
\hline $\mathrm{T}^{100_{2}}$ & .52 & - & - & .550 & .51 & - & .60 & - & .54 \\
\hline mo & .137 & .140 & .138 & - & 139 & .145 & .140 & - & $.140 \mathrm{c}$ \\
\hline & & & & in parts $p$ & illiton & & & & \\
\hline
\end{tabular}

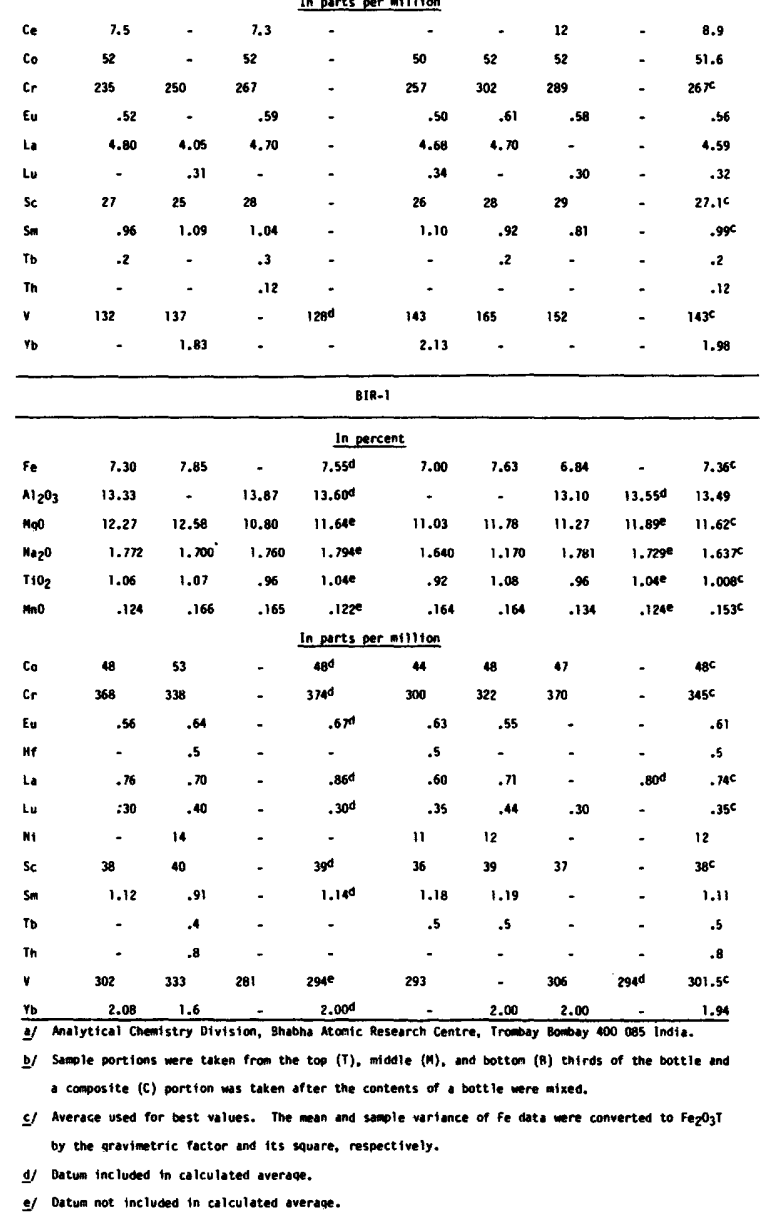



